\title{
PROPAGATION OF SINGULARITIES IN MANY-BODY SCATTERING
}

\author{
BY ANDRÁS VASY ${ }^{1}$
}

ABSTRACT. - In this paper we describe the propagation of singularities of tempered distributional solutions $u \in \mathcal{S}^{\prime}$ of $(H-\lambda) u=0, \lambda>0$, where $H$ is a many-body Hamiltonian $H=\Delta+V, \Delta \geqslant 0$, $V=\sum_{a} V_{a}$, under the assumption that no subsystem has a bound state and that the two-body interactions $V_{a}$ are real-valued polyhomogeneous symbols of order -1 (e.g. Coulomb-type with the singularity at the origin removed). Here the term 'singularity' provides a microlocal description of the lack of decay at infinity. We use this result to prove that the wave front relation of the free-to-free S-matrix (which, under our assumptions, is all of the S-matrix) is given by the broken geodesic flow, broken at the 'singular directions', on $\mathbb{S}^{n-1}$ at time $\pi$. We also present a natural geometric generalization to asymptotically Euclidean spaces. (C) 2001 Éditions scientifiques et médicales Elsevier SAS

RÉSUMÉ. - Dans cet article on décrit la propagation des singularités des solutions tempérées $u \in \mathcal{S}^{\prime}$ de $(H-\lambda) u=0, \lambda>0$, où $H$ est un Hamiltonien à $N$ corps $H=\Delta+V, \Delta \geqslant 0, V=\sum_{a} V_{a}$, en supposant que les Hamiltoniens des sous-systèmes n'ont pas de vecteurs propres (dans $L^{2}$ ), et que les potentiels à deux corps $V_{a}$ sont polyhomogènes réels d'ordre -1 (par exemple, de type Coulomb, mais sans la singularité à l'origine). Ici la notion de "singularité" étudiée fournit une description microlocale de la croissance des fonctions à l'infini. On emploie ce résultat pour montrer que la relation de front d'onde de la matrice de diffusion relative aux $N$-amas (qui est la seule partie de la matrice de diffusion sous nos hypothèses) est donnée par le flot géodesique brisé dans les "directions singulières", sur $\mathbb{S}^{n-1}$ à temps $\pi$. On présente aussi une généralisation géométrique naturelle au cas des variétés asymptotiquement euclidiennes. (c) 2001 Éditions scientifiques et médicales Elsevier SAS

\section{Introduction}

In this paper we describe the propagation of singularities of generalized eigenfunctions of a many-body Hamiltonian $H=\Delta+V, \Delta$ the positive Laplacian, under the assumption that no subsystem has a bound state. We use this result to prove that the wave front relation of the freeto-free S-matrix (which is the only part of the S-matrix under our assumptions) is given by the broken geodesic flow, broken at the 'singular directions', on $\mathbb{S}^{n-1}$ at distance $\pi$. We remark that these results have been proved in three-body scattering, without the assumption on the absence of bound states, in [36]. Also, Bommier [1] and Skibsted [33] have shown that the kernels of the 2-cluster to free cluster and 2-cluster to 2-cluster S-matrices are smooth, and previously Isozaki had showed this in the three-body setting [14]. However, as is clear from the smoothness statement, the microlocal propagation picture that is crucial, for instance, in the discussion of free-tofree scattering, does not emerge in the previous examples when the initial state is a 2-cluster.

\footnotetext{
${ }^{1}$ Partially supported by NSF grant \#DMS-99-70607.

ANNALES SCIENTIFIQUES DE L'ÉCOLE NORMALE SUPÉRIEURE. - 0012-9593/01/03/@ 2001 Éditions scientifiques et médicales Elsevier SAS. All rights reserved
} 
In this section we discuss the setup in the Euclidean setting, but in the following ones we move to a natural geometric generalization introduced by Melrose in [22]. Namely, suppose that $X$ is a manifold with boundary equipped with a scattering metric $g$ and a cleanly intersecting family $\mathcal{C}$ of closed embedded submanifolds of $\partial X$ with $C_{0}=\partial X \in \mathcal{C}$. Thus, $g$ is a Riemannian metric in $\operatorname{int}(X)$ which is of the form $g=x^{-4} d x^{2}+x^{-2} h$ near $\partial X$; here $x \in \mathcal{C}^{\infty}(X)$ is a boundary defining function. We also assume that near every $p \in \partial X, \mathcal{C}$ is locally linearizable (i.e. in suitable coordinates near $p$, every element of $\mathcal{C}$ is linear); this holds if every element of $\mathcal{C}$ is totally geodesic with respect to some metric (not necessarily $h$ ) on $\partial X$. Let $\Delta$ be the positive Laplacian of $g$ and suppose that $V \in \mathcal{C}^{\infty}([X ; \mathcal{C}] ; \mathbb{R})$ vanishes at $\partial X \backslash \bigcup\{C \in \mathcal{C}: C \neq \partial X\}$, and $H=\Delta+V$ - we refer to Sections 2 and 6 for a more detailed discussion of the geometric and analytic aspects of the setup. We prove under the assumption that there are no bound states for each of the subsystems (we describe the assumption more precisely in Section 6, but it holds for example if $V \geqslant 0)$ that singularities of solutions $u \in \mathcal{C}^{-\infty}(X)$ of $(H-\lambda) u \in \dot{\mathcal{C}}^{\infty}(X)$ propagate along generalized broken bicharacteristics of $\Delta$ which are broken at $\mathcal{C}$. We also show that this implies a bound on the singularities of the kernel of the free-to-free S-matrix. In effect, we show that many-body scattering is in many respects a hyperbolic problem, much like the wave equation in domains with corners, for which the propagation of analytic singularities was proved by Lebeau [18]. The geometrically simpler setting, where the elements of $\mathcal{C}$ (except $C_{0}=\partial X$ ) are disjoint, corresponds to three-body scattering in the Euclidean setting, and then the analogy is with the wave equation in smoothly bounded domains, where the results for $\mathcal{C}^{\infty}$ singularities were proved by Melrose and Sjöstrand [23,24] and Taylor [34], and for analytic singularities by Sjöstrand [32].

Here however we caution that another important aspect of typical many-body systems is the presence of bound states of subsystems. While propagation theorems indicate that geometry plays a central role in scattering, bound states afford a similar role to spectral theory. Thus, in general, the two interact, even changing the characteristic set of the Hamiltonian. The generalized broken bicharacteristics are also more complicated in this setting, and, as a quick argument shows, the 'time $\pi$ ' part of our result will not hold if bound states are present. In addition, the Hamiltonian must possess additional structure (as the Euclidean ones do) so that propagation in bound states can be analyzed. Hence, in this paper, it is natural to impose our assumption that there are no bound states in the subsystems.

We emphasize that this assumption holds, for example, if $V \geqslant 0$. Indeed, then $H=\Delta+V \geqslant 0$, and by an argument generalizing the corresponding result of Froese and Herbst [4] in the Euclidean setting, $H$ has no positive eigenvalues. The proof of this fact in the geometric threebody setting can be found in [40], and it goes through in the geometric many-body setting since the potentials play a very minor role; they only enter via the Mourre estimate. Moreover, 0 cannot be an eigenvalue since for such an $L^{2}(d g)$ eigenfunction $u, 0=\langle(H+V) u, u\rangle=$ $\|d u\|_{L^{2}}+\langle u, V u\rangle$, which implies that $u$ is a constant, contradicting its $L^{2}$ behavior. (The argument is justified by elliptic regularity.)

We now return to the Euclidean setting. Before we can state the precise definitions, we need to introduce some basic (and mostly standard) notation. We consider the Euclidean space $\mathbb{R}^{n}$, and we assume that we are given a (finite) family $\mathcal{X}$ of linear subspaces $X_{a}, a \in I$, of $\mathbb{R}^{n}$ which is closed under intersections and includes the subspace $X_{1}=\{0\}$ consisting of the origin, and the whole space $X_{0}=\mathbb{R}^{n}$. Let $X^{a}$ be the orthocomplement of $X_{a}$, and let $\pi^{a}$ be the orthogonal projection to $X^{a}, \pi_{a}$ to $X_{a}$. A many-body Hamiltonian is an operator of the form

$$
H=\Delta+\sum_{a \in I}\left(\pi^{a}\right)^{*} V_{a}
$$


here $\Delta$ is the positive Laplacian, $V_{0}=0$, and the $V_{a}$ are real-valued functions in an appropriate class which we take here to be polyhomogeneous symbols of order -1 on the vector space $X_{a}$ to simplify the problem:

$$
V_{a} \in S_{\text {phg }}^{-1}\left(X^{a}\right) \text {. }
$$

In particular, smooth potentials $V_{a}$ which behave at infinity like the Coulomb potential are allowed. Since $\left(\pi^{a}\right)^{*} V_{a}$ is bounded and self-adjoint and $\Delta$ is self-adjoint with domain $H^{2}\left(\mathbb{R}^{n}\right)$ on $L^{2}=L^{2}\left(\mathbb{R}^{n}\right), H$ is also a self-adjoint operator on $L^{2}$ with domain $H^{2}\left(\mathbb{R}^{n}\right)$. We let $R(\lambda)=(H-\lambda)^{-1}$ for $\lambda \in \mathbb{C} \backslash \mathbb{R}$ be the resolvent of $H$.

There is a natural partial ordering on $I$ induced by the ordering of $X^{a}$ by inclusion. (Though the ordering based on inclusion of the $X_{a}$ would be sometimes more natural, and we use that for the geometric generalization of many-body scattering starting from the next section, here we use the conventional ordering.) Let $I_{1}=\{1\}$ (recall that $X_{1}=\{0\}$ ); 1 is the maximal element of $I$. A maximal element of $I \backslash I_{1}$ is called a 2-cluster; $I_{2}$ denotes the set of 2-clusters. In general, once $I_{k}$ has been defined for $k=1, \ldots, m-1$, we let $I_{m}$ (the set of $m$-clusters) be the set of maximal elements of $I_{m}^{\prime}=I \backslash \bigcup_{k=1}^{m-1} I_{k}$, if $I_{m}^{\prime}$ is not empty. If $I_{m}^{\prime}=\{0\}$ (so $I_{m+1}^{\prime}$ is empty), we call $H$ an $m$-body Hamiltonian. For example, if $I \neq\{0,1\}$, and for all $a, b \notin\{0,1\}$ with $a \neq b$ we have $X_{a} \cap X_{b}=\{0\}$, then $H$ is a 3-body Hamiltonian. The $N$-cluster of an $N$-body Hamiltonian is also called the free cluster, since it corresponds to the particles which are asymptotically free.

It is convenient to compactify these spaces as in [22]. Thus, we let $\mathbb{S}_{+}^{n}$ to be the radial compactification of $\mathbb{R}^{n}$ to a closed hemisphere, i.e. a ball (using the standard map RC given here in (2.3)), and $\mathbb{S}^{n-1}=\partial \mathbb{S}_{+}^{n}$. We write $w=r \omega, \omega \in \mathbb{S}^{n-1}$, for polar coordinates on $\mathbb{R}^{n}$, and we let $x \in \mathcal{C}^{\infty}\left(\mathbb{S}_{+}^{n}\right)$ be such that $x=\left(\mathrm{RC}^{-1}\right)^{*}\left(r^{-1}\right)$ for $r>1$. Hence, $x$ is a smoothed version of $r^{-1}$ (smoothed at the origin of $\mathbb{R}^{n}$ ), and it is a boundary defining function of $\mathbb{S}_{+}^{n}$. We usually identify (the interior of) $\mathbb{S}_{+}^{n}$ with $\mathbb{R}^{n}$. Thus, we write $S_{\mathrm{phg}}^{m}\left(\mathbb{S}_{+}^{n}\right)$ and $S_{\mathrm{phg}}^{m}\left(\mathbb{R}^{n}\right)$ interchangeably and we drop the explicit pull-back notation in the future and simply write $x=r^{-1}$ (for $r>1$ ). We also remark that we have

$$
S_{\text {phg }}^{m}\left(\mathbb{S}_{+}^{n}\right)=x^{m} \mathcal{C}^{\infty}\left(\mathbb{S}_{+}^{n}\right) .
$$

We recall that under RC, $\dot{\mathcal{C}}^{\infty}\left(\mathbb{S}_{+}^{n}\right)$, the space of smooth functions on $\mathbb{S}_{+}^{n}$ vanishing to infinite order at the boundary corresponds to the space of Schwartz functions $\mathcal{S}\left(\mathbb{R}^{n}\right)$, and its dual, $\mathcal{C}^{-\infty}\left(\mathbb{S}_{+}^{n}\right)$, to tempered distributions $\mathcal{S}^{\prime}\left(\mathbb{R}^{n}\right)$. We also have the following correspondence of weighted Sobolev spaces

$$
H_{\mathrm{sc}}^{k, l}\left(\mathbb{S}_{+}^{n}\right)=H^{k, l}=H^{k, l}\left(\mathbb{R}^{n}\right)=\langle w\rangle^{-l} H^{k}\left(\mathbb{R}^{n}\right)
$$

where $\langle w\rangle=\left(1+|w|^{2}\right)^{1 / 2}$. Thus, for $\lambda \in \mathbb{C} \backslash \mathbb{R}$ the resolvent extends to a map

$$
R(\lambda): H_{\mathrm{sc}}^{k, l}\left(\mathbb{S}_{+}^{n}\right) \rightarrow H_{\mathrm{sc}}^{k+2, l}\left(\mathbb{S}_{+}^{n}\right)
$$

Similarly, we let

$$
\bar{X}_{a}=\operatorname{cl}\left(\operatorname{RC}\left(X_{a}\right)\right), \quad C_{a}=\bar{X}_{a} \cap \partial \mathbb{S}_{+}^{n} .
$$

Hence, $C_{a}$ is a sphere of dimension $n_{a}-1$ where $n_{a}=\operatorname{dim} X_{a}$. We also let

$$
\mathcal{C}=\left\{C_{a}: a \in I\right\}
$$

Again, we write the polar coordinates on $X_{a}$ (with respect to the induced metric) as $w_{a}=r_{a} \omega_{a}$, $\omega_{a} \in C_{a}$, and let $x_{a}=r_{a}^{-1}$ (for $r_{a}>1$ ). We note that if $a$ is a 2-cluster then $C_{a} \cap C_{b}=\emptyset$ unless 
$b \leqslant a$. We also define the 'singular part' of $C_{a}$ as the set

$$
C_{a, \text { sing }}=\bigcup_{b \nless a}\left(C_{b} \cap C_{a}\right),
$$

and its 'regular part' as the set

$$
C_{a}^{\prime}=C_{a} \backslash \bigcup_{b \nless a} C_{b}=C_{a} \backslash C_{a, \text { sing }}
$$

For example, if $a$ is a 2-cluster then $C_{a, \text { sing }}=\emptyset$ and $C_{a}^{\prime}=C_{a}$. We sometimes write the coordinates on $X_{a} \oplus X^{a}$ as $\left(w_{a}, w^{a}\right)$.

Corresponding to each cluster $a$ we introduce the cluster Hamiltonian $h_{a}$ as an operator on $L^{2}\left(X^{a}\right)$ given by

$$
h_{a}=\Delta+\sum_{b \leqslant a} V_{b},
$$

$\Delta$ being the Laplacian of the induced metric on $X^{a}$. Thus, if $H$ is a $N$-body Hamiltonian and $a$ is a $k$-cluster, then $h_{a}$ is a $(N+1-k)$-body Hamiltonian. The $L^{2}$ eigenfunctions of $h_{a}$ play an important role in many-body scattering; we remark that by Froese's and Herbst's result, [4], $\operatorname{spec}_{p p}\left(h_{a}\right) \subset(-\infty, 0]$ (there are no positive eigenvalues). Moreover, $\operatorname{spec}_{p p}\left(h_{a}\right)$ is bounded below since $h_{a}$ differs from $\Delta$ by a bounded operator. Note that $X^{0}=\{0\}, h_{0}=0$, so the unique eigenvalue of $h_{0}$ is 0 .

The eigenvalues of $h_{a}$ can be used to define the set of thresholds of $h_{b}$. Namely, we let

$$
\Lambda_{a}=\bigcup_{b<a} \operatorname{spec}_{p p}\left(h_{b}\right)
$$

be the set of thresholds of $h_{a}$, and we also let

$$
\Lambda_{a}^{\prime}=\Lambda_{a} \cup \operatorname{spec}_{p p}\left(h_{a}\right)=\bigcup_{b \leqslant a} \operatorname{spec}_{p p}\left(h_{b}\right) .
$$

Thus, $0 \in \Lambda_{a}$ for $a \neq 0$ and $\Lambda_{a} \subset(-\infty, 0]$. It follows from the Mourre theory (see e.g. [5,27]) that $\Lambda_{a}$ is closed, countable, and $\operatorname{spec}_{p p}\left(h_{a}\right)$ can only accumulate at $\Lambda_{a}$. Moreover, $R(\lambda)$, considered as an operator on weighted Sobolev spaces, has a limit

$$
R(\lambda \pm i 0): H_{\mathrm{sc}}^{k, l}\left(\mathbb{S}_{+}^{n}\right) \rightarrow H_{\mathrm{sc}}^{k+2, l^{\prime}}\left(\mathbb{S}_{+}^{n}\right)
$$

for $l>1 / 2, l^{\prime}<-1 / 2$, from either half of the complex plane away from

$$
\Lambda=\Lambda_{1} \cup \operatorname{spec}_{p p}(H) .
$$

In addition, $L^{2}$ eigenfunctions of $h_{a}$ with eigenvalues which are not thresholds are necessarily Schwartz functions on $X^{a}$ (see [4]). We also label the eigenvalues of $h_{a}$, counted with multiplicities, by integers $m$, and we call the pairs $\alpha=(a, m)$ channels. We denote the eigenvalue of the channel $\alpha$ by $\varepsilon_{\alpha}$, write $\psi_{\alpha}$ for a corresponding normalized eigenfunction, and let $e_{\alpha}$ be the orthogonal projection to $\psi_{\alpha}$ in $L^{2}\left(X^{a}\right)$.

The definition of the free-to-free S-matrix we consider comes from the stationary theory, more precisely from the asymptotic behavior of generalized eigenfunctions, see [35], and cf. [22,40]. 
Apart from the difference in normalization, it is the same as the S-matrix given by the wave operators, see [39]. For simplicity, we state the asymptotic expansion under the assumption that $V_{a}$ is polyhomogeneous of order -2 (so it decays as $\left|w^{a}\right|^{-2}$ ). Namely, for $\lambda \in(0, \infty)$ and $g \in \mathcal{C}_{c}^{\infty}\left(C_{0}^{\prime}\right)$, there is a unique $u \in \mathcal{C}^{-\infty}\left(\mathbb{S}_{+}^{n}\right)$ (i.e. $\left.u \in \mathcal{S}^{\prime}\left(\mathbb{R}^{n}\right)\right)$ such that $(H-\lambda) u=0$, and $u$ has the form

$$
u=\mathrm{e}^{-i \sqrt{\lambda} r} r^{-(n-1) / 2} v_{-}+R(\lambda+i 0) f,
$$

where $v_{-} \in \mathcal{C}^{\infty}\left(\mathbb{S}_{+}^{n}\right),\left.v_{-}\right|_{\mathbb{S}^{n-1}}=g$, and $f \in \dot{\mathcal{C}}^{\infty}\left(\mathbb{S}_{+}^{n}\right)$. In addition, this $u$ is of the form

$$
u=\mathrm{e}^{-i \sqrt{\lambda} r} r^{-(n-1) / 2} v_{-}+\mathrm{e}^{i \sqrt{\lambda} r} r^{-(n-1) / 2} v_{+}, \quad v_{+} \in \mathcal{C}^{\infty}\left(\mathbb{S}_{+}^{n} \backslash C_{0, \text { sing }}\right)
$$

The Poisson operator with free initial data is the operator

$$
P_{0,+}(\lambda): \mathcal{C}_{c}^{\infty}\left(C_{0}^{\prime}\right) \rightarrow \mathcal{C}^{-\infty}\left(\mathbb{S}_{+}^{n}\right), \quad P_{0,+}(\lambda) g=u .
$$

Following [35], we define the free-to-free scattering matrix, $S_{00}(\lambda)$ as the map

$$
\begin{gathered}
S_{00}(\lambda): \mathcal{C}_{c}^{\infty}\left(C_{0}^{\prime}\right) \rightarrow \mathcal{C}^{\infty}\left(C_{0}^{\prime}\right), \\
S_{00}(\lambda) g=\left.v_{+}\right|_{C_{0}^{\prime}},
\end{gathered}
$$

so it relates the incoming amplitude $\left.v_{-}\right|_{\mathbb{S}^{n-1}}$ to the outgoing one, $\left.v_{+}\right|_{\mathbb{S}^{n-1}}$. We recall from [39] that the wave operator free-to-free $\mathrm{S}$-matrix is then given by $i^{n-1} S_{00}(\lambda) R$ (as maps $\left.\mathcal{C}_{c}^{\infty}\left(C_{0}^{\prime}\right) \rightarrow \mathcal{C}^{-\infty}\left(C_{0}^{\prime}\right)\right)$ where $R$ is pull back by the antipodal map on $C_{0}$.

There are only minor changes if $V_{a}$ is polyhomogeneous of order -1 . Namely, the asymptotic expansions in (1.15) and (1.16) must be replaced by

$$
\begin{gathered}
\mathrm{e}^{ \pm i \sqrt{\lambda} r} r^{-i \alpha_{ \pm}-(n-1) / 2} v_{ \pm}, \quad \alpha_{ \pm}=\alpha_{ \pm, \lambda}= \pm\left. V^{\prime}\right|_{C_{0}^{\prime}} / 2 \sqrt{\lambda} \in \mathcal{C}^{\infty}\left(C_{0}^{\prime}\right), \quad V=x V^{\prime} \\
v_{ \pm} \sim \sum_{j=0}^{\infty} \sum_{s \leqslant 2 j} a_{j, s, \pm}(\omega) r^{-j}(\log r)^{s}, \quad a_{j, s,-} \in \mathcal{C}_{c}^{\infty}\left(C_{0}^{\prime}\right), a_{j, s,+} \in \mathcal{C}^{\infty}\left(C_{0}^{\prime}\right) .
\end{gathered}
$$

Note that $\alpha_{ \pm}$are not defined at $C_{0, \text { sing }}$, but that does not cause any problems even in the uniqueness statement, (1.15), since $v_{-}$vanishes at $\mathbb{S}^{n-1}$ near $C_{0 \text {,sing }}$ to infinite order.

Our main theorem describes the structure of $S_{00}(\lambda)$. We first introduce the broken geodesic flow (of the standard Riemannian metric $h$ ) on $\mathbb{S}^{n-1}$, broken at $\mathcal{C}$. We denote by $S \mathbb{S}^{n-1}$ the sphere bundle of $\mathbb{S}^{n-1}$ identified as the unit-length subbundle of $T \mathbb{S}^{n-1}$ with respect to $h$. Let $I=[\alpha, \beta] \subset \mathbb{R}$ be an interval. We say that a curve $\gamma: I \rightarrow \mathbb{S}^{n-1}$ is a broken geodesic of $h$, broken at $\mathcal{C}$, if two conditions are satisfied. First, there exists a finite set of points $t_{j} \in I$, $\alpha=t_{0}<t_{1}<\cdots<t_{k-1}<t_{k}=\beta$ such that for each $j,\left.\gamma\right|_{\left[t_{j}, t_{j+1}\right]}$ is a geodesic of $h$, and for all $t \in\left(t_{j}, t_{j+1}\right), \gamma^{\prime}(t) \in S \mathbb{S}^{n-1}$. Second, for all $j$, if $\gamma\left(t_{j}\right) \in C_{a}^{\prime}$ then the limits $\gamma^{\prime}\left(t_{j}-0\right)$ and $\gamma^{\prime}\left(t_{j}+0\right)$ both exist and differ by a vector in $T_{\gamma\left(t_{j}\right)} \mathbb{S}^{n-1}$ which is orthogonal to $T_{\gamma\left(t_{j}\right)} C_{a}$ (i.e. the usual law of reflection is satisfied; see Fig. 1). We say that $p, q \in S \mathbb{S}^{n-1}$ are related by the broken geodesic flow at time $\pi$ if there is a broken geodesic $\gamma$ defined on $[0, \pi]$, such that $\gamma^{\prime}(0)=p, \gamma^{\prime}(\pi)=q$. Using the metric $h$ to identify $S \partial X$ and $S^{*} \partial X$, this defines the broken geodesic 'flow' at time $\pi$ on $S^{*} \partial X$. We refer to Definition 6.6 and Section 7 for a more complete discussion. We then have the following result: 


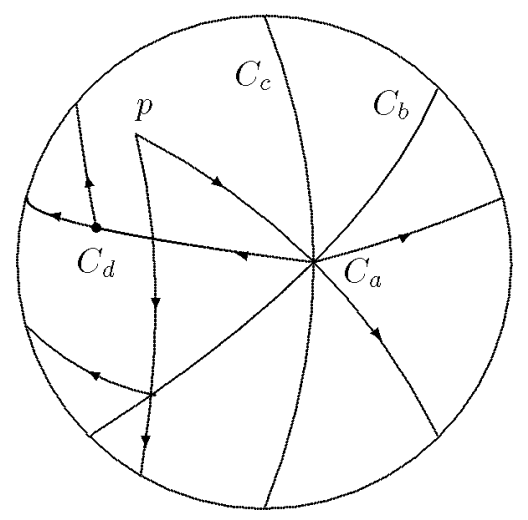

Fig. 1. Broken geodesics on $\mathbb{S}^{2}$ starting at $p$. Here $C_{a}=C_{b} \cap C_{c}$.

THEOREM. - Suppose that no subsystem of $H$ has bound states, i.e. for $a \neq 0, \operatorname{spec}_{p p}\left(h_{a}\right)=\emptyset$. Then the free-to-free scattering matrix, $S_{00}(\lambda)$, extends to a continuous linear map $\mathcal{C}_{c}^{-\infty}\left(C_{0}^{\prime}\right) \rightarrow \mathcal{C}^{-\infty}\left(C_{0}^{\prime}\right)$. The wave front relation of $S_{00}(\lambda)$ is given by the broken geodesic flow at time $\pi$.

In the actual many-body problem, $w \in X_{a}$ means that several particles are close to each other, namely the ones corresponding to the cluster decomposition $a$. Thus, $\omega \in C_{a}$ is a statement that the particles corresponding to cluster $a$ collide. Hence, the Theorem describes how many-body scattering can be understood, modulo smoothing (hence in the $\mathcal{C}^{\infty}$ sense trivial) terms, as a sequence of a finite number of collisions involving the particles. Namely, each 'break' $t_{j}$ in the broken geodesic describes a collision involving the cluster decomposition $a$. In the three-body setting with Schwartz potentials it was shown in [37] that the amplitude of the reflected wave is given, to top order, by the corresponding 2-body S-matrix; an analogous statement also holds for short-range potentials. In particular, this shows that the Theorem is sharp as far as the location of singularities is concerned.

We also remark that in the Euclidean setting, unbroken geodesic flow to distance $\pi$ amounts to pull-back by the antipodal map on $\mathbb{S}^{n-1}=\partial \mathbb{S}_{+}^{n}$, so it corresponds to free propagation: particles leave in the direction opposite to the one from which they entered.

Our approach to proving this theorem is via the analysis of generalized eigenfunctions of $H$, i.e. of $u \in \mathcal{C}^{-\infty}\left(\mathbb{S}_{+}^{n}\right)$ satisfying $(H-\lambda) u=0$. We prove that 'singularities' of generalized eigenfunctions of $H$ propagate along broken bicharacteristics in the characteristic set of $H$, similarly to singularities of the solutions of the wave equation. Here 'singularities' are not understood as the lack of smoothness: indeed $H$ is elliptic in the usual sense, so every generalized eigenfunction is $\mathcal{C}^{\infty}$ in the interior of $\mathbb{S}_{+}^{n}$, i.e. on $\mathbb{R}^{n}$. Instead, in this situation singularity means the lack of rapid decay $u$. Correspondingly, we define a wave front set, $\operatorname{WF}_{\mathrm{Sc}}(u)$, at infinity, i.e. at $\partial \mathbb{S}_{+}^{n}$, and we will prove its invariance under the broken bicharacteristic flow.

The two notions of singularities are very closely related via the Fourier transform. Here for simplicity consider $\Delta-\lambda$ in place of $H-\lambda$. If $(\Delta-\lambda) u=0$, then the Fourier transform of $u, \mathcal{F} u$, satisfies $\left(|\xi|^{2}-\lambda\right) \mathcal{F} u=0$ where $\xi$ is the dual variable of $w$. Now, the multiplication operator $P=|\xi|^{2}-\lambda$ can be regarded as a 0th order differential operator. Hence, by Hörmander's theorem, see e.g. [12], $\mathrm{WF}(\mathcal{F} u)$ is invariant under the bicharacteristic flow in the characteristic variety of $P$, i.e. in the set $\left\{\left(\xi, \xi^{*}\right):|\xi|^{2}-\lambda=0\right\}$ where we have written $\xi^{*}$ for the dual variable of $\xi$, so $\xi^{*}$ is in fact $w$. Moreover, in the two-body problem, i.e. if $V$ is a symbol (of say order -1$)$ on $\mathbb{R}^{n}, H=\Delta+V$, and if $(H-\lambda) u=0$, we still have $P \mathcal{F} u=0$ where now 
$P=|\xi|^{2}-\lambda+\mathcal{F} V \mathcal{F}^{-1}$. Since $V$ is a symbol of order $-1, \mathcal{F} V \mathcal{F}^{-1}$ is a pseudo-differential operator of order -1 , hence lower order than $|\xi|^{2}-\lambda$. Thus, the principal symbol of $P$ is still $|\xi|^{2}-\lambda$ (recall that $\xi^{*}$ is the cotangent variable, so this is indeed homogeneous of order 0 in $\xi^{*}-$ it is independent of $\xi^{*}$ ). Hence, Hörmander's theorem is applicable and we have the same propagation statement as before.

In the two-body setting the relevant wave front set measuring lack of decay at infinity is the scattering one, $\mathrm{WF}_{\mathrm{sc}}$. For $u \in \mathcal{S}^{\prime}\left(\mathbb{R}^{n}\right), \mathrm{WF}_{\mathrm{sc}}(u)$ is essentially given by the usual wave front set of the Fourier transform of $u$, i.e. by $\operatorname{WF}(\mathcal{F} u)$, after interchanging the role of the base and dual variables. Since the Fourier transform interchanges decay at infinity and smoothness, $\operatorname{WF}(\mathcal{F} u)$ indeed measures the decay of $u$ at infinity in a microlocal sense. Hence, Hörmander's propagation theorem translated directly into a propagation theorem for $\mathrm{WF}_{\mathrm{sc}}(u)$. This result was described by Melrose in [22] where he introduced the notion of $\mathrm{WF}_{\mathrm{sc}}$.

In the many-body setting conjugation by the Fourier transform is much less convenient. Hence, we will design an appropriate microlocal way of measuring the lack of decay at infinity without resorting to the Fourier transform. Instead, we introduce an algebra of many-body pseudodifferential operators $\Psi_{\mathrm{Sc}}\left(\mathbb{S}_{+}^{n}, \mathcal{C}\right)$ which reflects the geometry, and use it to define the wave front set at infinity. We remark, however, that for (approximate) generalized eigenfunctions of $H$, a characterization based on the Fourier transform is possible; see Remark 5.3 and Proposition 5.5. We then prove a propagation of singularities theorem for generalized eigenfunctions of manybody Hamiltonians $H$; here 'singularities' are understood in the sense of the new wave front set at infinity. The proof of this theorem is via a microlocal positive commutator estimate, similarly to the proof of Hörmander's theorem, or indeed to the proof of the propagation theorems for $\mathcal{C}^{\infty}$ singularities of solutions of the wave equation with domains with boundaries [23]. Finally, we relate such a result to the structure of the S-matrix. This step is comparatively easy as indicated by our description of the S-matrix in terms of generalized eigenfunctions of $H$.

Positive commutator estimates have also played a major role in many-body scattering starting with the work of Mourre [26], Perry, Sigal and Simon [27], Froese and Herbst [5], Jensen [17], Gérard, Isozaki and Skibsted [6,7] and Wang [41]. In particular, the Mourre estimate is one of them; it estimates $i\left[H, w \cdot D_{w}+D_{w} \cdot w\right]$. This and some other global positive commutator results have been used to prove the global results mentioned in the first paragraph about some of the S-matrices with initial state in a two-cluster. They also give the basis for the existence, uniqueness and equivalence statements in our definition of the S-matrix by asymptotic expansions; these statements are discussed in [39] in more detail. Correspondingly, these global estimates will appear in Sections 11-12 of this paper where we turn the propagation results for generalized eigenfunctions into statements about the S-matrix.

We remark that the wave-operator approach defines the S-matrix as a bounded operator

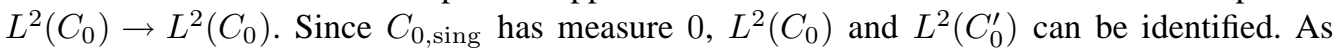
$\mathcal{C}_{c}^{\infty}\left(C_{0}^{\prime}\right)$ is dense in $L^{2}\left(C_{0}^{\prime}\right)$, the asymptotic expansion S-matrix $S_{00}(\lambda)$ indeed determines the wave-operator one.

The propagation of singularities of generalized eigenfunctions of $H$ is determined by the principal part of $H$; terms decaying at the boundary do not change the analysis. As opposed to this, the precise structure of incoming and outgoing functions, $R(\lambda \pm i 0) f, f \in \dot{\mathcal{C}}^{\infty}\left(\mathbb{S}_{+}^{n}\right)$, depends on lower order terms; a relatively trivial example is given by the appearence of $r^{-i \alpha_{ \pm}}$ in (1.20) for long-range potentials. Since we consider $S_{00}(\lambda)$ and $P_{0,+}(\lambda)$ as operators on

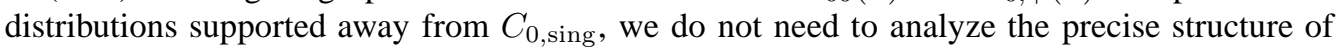
incoming/outgoing functions at $C_{0, \text { sing, }}$, which is not 'principal type', although we certainly analyze the propagation of singularities there. Thus, we do not discuss what happens when the support of the incoming scattering data increases to $C_{0}^{\prime}$, even if the data are $L^{2}$. But the behavior of $P_{0,+}(\lambda)$, as the support of the data increases to $C_{0}^{\prime}$, plays an important part in asymptotic 
completeness, which states that all possible outcomes of a scattering experiment are indeed described by a combination of bound states of the cluster Hamiltonians, with asymptotically free motion in the intercluster variables. Thus, our results cannot be used directly to supply a proof of asymptotic completeness. This completeness property of many-body Hamiltonians was proved by Sigal and Soffer, Graf, Dereziński and Yafaev under different assumptions on the potentials and by different techniques [28,29,31,30,8,2,42]. In particular, Yafaev's paper [42] shows quite explicitly the importance of the special structure of the Euclidean Hamiltonian. This structure enables him to obtain a positive commutator estimate, which would not follow from our indicial operator arguments in Section 9, and which is then used to prove asymptotic completeness.

Finally we comment on the requirement that the collection $\mathcal{C}$ be locally linearizable. We show in the next section that it is equivalent to the existence of a neighborhood of every point $p \in \partial X$ and a metric on it, in terms of which all elements of $\mathcal{C}$ are totally geodesic. The importance of this assumption is closely related to the existence of a sufficient number of smooth vector fields on $\partial X$ which are tangent to every element of $\mathcal{C}$. Such smooth vector fields always exist once we resolve the geometry of $\mathcal{C}$, i.e. on the blown-up space $[\partial X ; \mathcal{C}]$, but in general, without our assumption, there are not enough such smooth vector fields on $\partial X$. In the first part of the paper, we discuss the pseudo-differential algebra associated to many-body scattering. For this purpose we need to blow up $\mathcal{C}$, in part for analyzing the indicial operators (see the following paragraph). Thus, in this part of the paper, the issue of local linearizability is irrelevant, and we do not assume it. However, in the second part of the paper, the geometry of the compressed cotangent bundle, the discussion of generalized broken bicharacteristics and the construction of the positive commutators would all be more complicated without it, so from Section 5 on, we assume the local linearizability of $\mathcal{C}$.

This paper is organized as follows. In the next section we describe the geometric generalization of the many-body problem which was outlined above. This includes a discussion of many-body geometry and the definition of many-body differential operators. In Section 3 we proceed to define and analyze the corresponding algebra of pseudo-differential operators, $\Psi_{\mathrm{Sc}}(X, \mathcal{C})$, which reflects this geometry. It includes many-body Hamiltonians, as well as their resolvent away from the real axis. It extends the definition of the three-body calculus presented in [40], though here we emphasize the definition of the calculus via localization and quantization as opposed to the conormal description of the kernels on an appropriate resolved space. In Section 4 we construct the indicial operators in this calculus. They provide a non-commutative analog of the principal symbol in standard microlocal analysis. Our proof of positivity in commutator estimates is based on replacing the argument of Froese and Herbst [5] by indicial operator techniques. In Section 5 we define the wave front set at infinity, $\mathrm{WF}_{\mathrm{Sc}}(u)$, corresponding to the many-body geometry and pseudo-differential operators. The definition given here differs from the one in [40]; it follows the fibred cusp definition of Mazzeo and Melrose [19]. These definitions, however, give the same result for approximate generalized eigenfunctions of $H$.

In Section 6 we discuss many-body type Hamiltonians and their generalized broken bicharacteristics. This section is, to a significant degree, based on Lebeau's paper [18]. In Section 7 we give a much more detailed description of the generalized broken bicharacteristics in the case when all elements $C \in \mathcal{C}$ are totally geodesic. Of course, this is true in the Euclidean setting. In Sections 8-9 we build the technical tools for turning a symbolic positive commutator calculation into an operator estimate. In Section 10 we prove that singularities of generalized eigenfunctions of many-body type Hamiltonians propagate along generalized broken bicharacteristics. This is the main new result of the paper. In Sections 11-12 we use this and adaptations of the global estimates, in particular those of Gérard, Isozaki and Skibsted [6,7], to analyze the structure of the resolvent and that of the scattering matrix. Finally, in the Appendix we prove some of the results quoted from Lebeau's paper, using slightly different methods. 
The propagation estimates of Section 10 lie at the heart of this paper. The reader may want to skip some of the technical sections when reading the paper for the first time. It may be useful to keep Mourre-type estimates and especially their microlocalized versions as in $[6,7]$ in mind while reading Section 10.

I would like to thank Richard Melrose for suggesting this problem to me (in the three-body setting) as my $\mathrm{PhD}$ thesis problem and for our very fruitful discussions. His firm belief that scattering theory can be understood in microlocal terms similar to the well-known theory of hyperbolic operators motivated me both during my $\mathrm{PhD}$ work [36] and while working on its extension that appears in this paper. I am grateful to Maciej Zworski for introducing me to the work of Gilles Lebeau [18], for many helpful discussions and for his encouragement. It was Lebeau's paper that convinced me that the results presented here were within reach, and it plays a particularly central role in Section 6 where the generalized broken bicharacteristics are described. I would also like to thank the referee for the careful reading of the original manuscript and for the resulting numerous improvements. I am also grateful to Andrew Hassell, Rafe Mazzeo, Erik Skibsted and Jared Wunsch for helpful discussions, their encouragement and for their interest in this research.

\section{Many-body geometry and differential operators}

It is convenient to carry out the construction in the general geometric setting. We first describe the many-body geometry.

Thus, let $X$ be a compact manifold with boundary, and let

$$
\mathcal{C}=\left\{C_{a}: a \in I\right\}
$$

be a finite set of closed embedded submanifolds of $\partial X$ such that $\partial X=C_{0} \in \mathcal{C}$ and for all $a, b \in I$ either $C_{a}$ and $C_{b}$ are disjoint, or they intersect cleanly and $C_{a} \cap C_{b}=C_{c}$ for some $c \in I$. We introduce a partial order on $\mathcal{C}$ given by inclusion on $\mathcal{C}$, namely

$$
C_{a} \leqslant C_{b} \text { if and only if } C_{a} \subset C_{b} .
$$

This partial order is the opposite of the partial order used traditionally in many-body scattering, discussed in the introduction, but it will be more convenient for us since it simply corresponds to inclusion. A chain is defined as usual as a set on which $<$ gives a linear order.

Definition 2.1. - Let $X$ and $\mathcal{C}$ be as above. We say that $(X, \mathcal{C})$ is a space with $N$-body geometry (or an $N$-body space), $N \geqslant 2$, if the maximal length of chains is $N-1$. Similarly, we say that $C_{a}$ is a $k$-cluster if the maximal length of chains whose maximal element is $C_{a}$ is $k-1$. We also say that $(X, \mathcal{C})$ is a many-body space if we do not wish to specify $N$.

Thus, if $C_{a}$ is minimal, it is a 2-cluster, and if $(X, \mathcal{C})$ is a space with $N$-body geometry then $\partial X$ is an $N$-cluster. The numerology is chosen here so that we conform to the usual definitions in Euclidean many-body scattering, described in the Introduction.

Before defining the algebra of many-body scattering differential operators on $(X, \mathcal{C})$, we discuss the simultaneous local linearizability of the collection $\mathcal{C}$. As we have mentioned in the Introduction, the analysis of generalized broken geodesics as well as the commutator constructions of this paper become simpler if $\mathcal{C}$ is locally linearizable. To make this notion precise, we make the following definition.

DEFINITION 2.2. - We say that a many-body space $(X, \mathcal{C})$ is locally linearizable (or is locally trivial) if for every $p \in \partial X$ there exists a diffeomorphism $\phi$ from a neighborhood $U$ of $p$ in $\partial X$ 
to a neighborhood $U^{\prime}$ of the origin of a vector space $V$ such that for each $C \in \mathcal{C}$, the image of $C \cap U$ under $\phi$ is the intersection of a linear subspace of $V$ with $U^{\prime}$.

Remark 2.3. - In three-body type geometry, where the elements of $\mathcal{C}$ except $C_{0}$ are disjoint, $(X, \mathcal{C})$ is automatically locally linearizable. The same holds, essentially by definition, if $\mathcal{C}$ is a normal collection, see [20, Chapter V].

Local triviality of $\mathcal{C}$ is closely related to the question whether every element of $\mathcal{C}$ is locally totally geodesic with respect to some metric. In fact,

LEMMA 2.4. - A many-body space $(X, \mathcal{C})$ is locally linearizable if and only if every $p \in \partial X$ has a neighborhood $U$ in $\partial X$ and a Riemannian metric $h_{U}$ on $U$ such that for each element $C$ of $\mathcal{C}, C \cap U$ is totally geodesic with respect to $h_{U}$.

Proof. - Suppose first that $p \in \partial X$ and $U, h_{U}$ are as above. By shrinking $U$ if necessary, we can make sure that $p \notin C$ implies $C \cap U=\emptyset$ for every $C \in \mathcal{C}$. By shrinking $U$ further if necessary, we can arrange that the exponential map of $h_{U}$ at $p \in \partial X$ identifies a neighborhood $U^{\prime}$ of the origin in $V=T_{p} \partial X$ and $U$. Moreover, the elements $C \in \mathcal{C}$ for which $p \in C$, are identified with $T_{p} C \cap U^{\prime}$, since these $C$ are totally geodesic. This proves that $(X, \mathcal{C})$ is locally linearizable.

Conversely, if $(X, \mathcal{C})$ is locally linearizable, then the choice of an inner product on $V$ induces a metric on $T V$, hence on $U$ via the diffeomorphism $\phi$, and as linear subspaces of $V$ are totally geodesic with respect to this metric on $T V$, the same holds for $\mathcal{C}$ over $U$.

After this brief discussion on the local linearizability of $\mathcal{C}$, we turn to the setting of most interest, namely to Euclidean many-body geometry. Suppose that $X=\mathbb{S}_{+}^{n}$ is the radial compactification of $\mathbb{R}^{n}$ and $\mathcal{X}$ is a family of linear subspaces of $\mathbb{R}^{n}$ as discussed in the introduction. Recall from [22] that $\mathrm{RC}: \mathbb{R}^{n} \rightarrow \mathbb{S}_{+}^{n}$ is given by

$$
\mathrm{RC}(w)=\left(1 /\left(1+|w|^{2}\right)^{1 / 2}, w /\left(1+|w|^{2}\right)^{1 / 2}\right) \in \mathbb{S}_{+}^{n} \subset \mathbb{R}^{n+1}, \quad w \in \mathbb{R}^{n} .
$$

Here we use the notation RC instead of SP, used in [22], to avoid confusion with the standard stereographic projection giving a one-point compactification of $\mathbb{R}^{n}$. We write the coordinates on $\mathbb{R}^{n}=X_{a} \oplus X^{a}$ as $\left(w_{a}, w^{a}\right)$. Let $m=\operatorname{dim} X_{a}$. We again let

$$
\bar{X}_{a}=\operatorname{cl}\left(\operatorname{RC}\left(X_{a}\right)\right), \quad C_{a}=\bar{X}_{a} \cap \partial \mathbb{S}_{+}^{n} .
$$

We next show that polyhomogeneous symbols on $X^{a}$, pulled back to $\mathbb{R}^{n}$ by $\pi^{a}$, are smooth on the blown-up space $\left[X ; C_{a}\right]$. Recall that the blow-up process is simply an invariant way of introducing polar coordinates about a submanifold. A full description appears in [20] and a more concise one in [22, Appendix A], but we give a brief summary here. Thus, suppose that $X$ is a manifold with corners and $C$ is a p-submanifold (i.e. product submanifold) of $\partial X$. Thus, near any $p \in C$ we have local coordinates $x_{i}(i=1, \ldots, r), y_{j}(j=1, \ldots, n-r), n=\operatorname{dim} X$, such that the boundary hypersurfaces of $X$ through $p$ are defined by $x_{i}=0, X$ is given by $x_{i} \geqslant 0$, $i=1, \ldots, r$, and such that $C$ is locally defined by the vanishing of certain of these coordinates, e.g. by $x_{i}=0, i=1, \ldots s, s \leqslant r$, and $y_{j}=0, j=1, \ldots, p, p \leqslant n-r$. A tangent vector $V \in T_{q} X$, $q$ near $p$, is inward-pointing if $\left(V x_{i}\right)(q) \geqslant 0$ for all $i$. The normal bundle of $C$ is the quotient bundle

$$
N C=T_{C} X / T C .
$$

The inward pointing normal bundle of $C, N^{+} C$, is the image of $T^{+} X$, consisting of inward pointing tangent vectors, in $N C$. Thus, near $p, X$ is diffeomorphic to the inward-pointing normal 
bundle of $C$. The blow-up of $X$ along $C$ is locally defined as the blow up of the 0 section of $N^{+} C$, i.e. by introducing the new $\mathcal{C}^{\infty}$ structure in $N^{+} C$ given by polar coordinates in the fibers of the bundle and by the base coordinates pulled back from $C$. While this construction depends on some choices, the resulting $\mathcal{C}^{\infty}$ structure does not. The blow-up of $X$ along $C$ is denoted by $[X ; C]$. The blow-down map $[X ; C] \rightarrow X$ is the smooth map corresponding to expressing standard coordinates on a vector space, $N_{q}^{+} C$, in terms of polar coordinates. It is denoted by $\beta[X ; C]$. The front face of the blow-up is the inverse image of $C$ (i.e. of the zero section of $N^{+} C$ ) under $\beta[X ; C]$. Hence, it is a bundle over $C$ whose fibers are the intersection of a sphere with a 'quadrant' corresponding to the inward-pointing condition, i.e. to $x_{i} \geqslant 0$. In fact, it is the inward pointing sphere bundle $S^{+} N C$ which is the quotient of $N^{+} C \backslash o, o$ denoting the zero section, by the natural $\mathbb{R}^{+}$actions in its fibers.

We again return to the Euclidean setting. In particular $X=\mathbb{S}_{+}^{n}$. We denote the blow-down map by $\beta\left[X ; C_{a}\right]:\left[X ; C_{a}\right] \rightarrow X$. Now $S^{+} N C_{a}$ is a hemisphere bundle over $C_{a}$, which can be identified with the radial compactification of the normal bundle of $C_{a}$ in $\partial X$ whose fibers can in turn be identified with $X^{a}$. To see this in more concrete terms, we proceed by finding local coordinates on $\left[X ; C_{a}\right]$ explicitly. It is convenient to do so by using projective coordinates rather than the standard polar coordinates. Near $C_{a}$ in $\mathbb{S}_{+}^{n}$ we have $\left|w_{a}\right|>c\left|w^{a}\right|$ for some $c>0$. Hence, near any point $p \in C_{a}$ one of the coordinate functions $\left(w_{a}\right)_{j}$ which we may take to be $\left(w_{a}\right)_{m}$, satisfies $\left|\left(w_{a}\right)_{m}\right|>c^{\prime}\left|\left(w_{a}\right)_{j}\right|,\left|\left(w_{a}\right)_{m}\right|>c^{\prime}\left|w^{a}\right|$ for some $c^{\prime}>0$. Taking into account the coordinate form of $\mathrm{RC}$ we see that

$$
\begin{aligned}
& x=\left|\left(w_{a}\right)_{m}\right|^{-1}, \quad z_{j}=\frac{\left(w_{a}\right)_{j}}{\left|\left(w_{a}\right)_{m}\right|} \quad(j=1, \ldots, m-1), \\
& y_{j}=\frac{\left(w^{a}\right)_{j}}{\left|\left(w_{a}\right)_{m}\right|} \quad(j=1, \ldots, n-m)
\end{aligned}
$$

give coordinates on $\mathbb{S}_{+}^{n}$ near $p$. In these coordinates $C_{a}$ is defined by $x=0, y=0$. Correspondingly, we have coordinates

$$
x, \quad z_{j} \quad(j=1, \ldots, m-1), \quad Y_{j}=y_{j} / x \quad(j=1, \ldots, n-m),
$$

i.e.

$x=\left|\left(w_{a}\right)_{m}\right|^{-1}, \quad z_{j}=\frac{\left(w_{a}\right)_{j}}{\left|\left(w_{a}\right)_{m}\right|} \quad(j=1, \ldots, m-1), \quad Y_{j}=\left(w^{a}\right)_{j} \quad(j=1, \ldots, n-m)$

near the interior of the front face $\mathrm{ff}$ of the blow-up $\left[X ; C_{a}\right]$, i.e. near the interior of $\mathrm{ff}=$ $\beta\left[X ; C_{a}\right]^{*} C_{a}$; see Fig. 2 .

Near the corner $\partial \beta\left[X ; C_{a}\right]^{*} C_{a}=\beta\left[X ; C_{a}\right]^{*} C_{a} \cap \beta\left[X ; C_{a}\right]^{*} \partial X$, in the lift of the region defined for some $k$ by $\left|y_{k}\right| \geqslant c\left|y_{j}\right|$ for some $c>0$ and all $j \neq k$,

$$
\hat{x}=x / y_{k}, \quad \hat{Y}_{j}=y_{j} / y_{k} \quad(j \neq k), \quad y_{k}, \quad z
$$

give coordinates. In terms of the original Euclidean variables these are

$$
\begin{gathered}
\hat{x}=\left|\left(w^{a}\right)_{k}\right|^{-1}, \quad z_{j}=\frac{\left(w_{a}\right)_{j}}{\left|\left(w_{a}\right)_{m}\right|} \quad(j=1, \ldots, m-1), \\
\hat{Y}_{j}=\frac{\left(w^{a}\right)_{j}}{\left(w^{a}\right)_{k}} \quad(j=1, \ldots, n-m, j \neq k), \quad y_{k}=\frac{\left(w^{a}\right)_{k}}{\left|\left(w_{a}\right)_{m}\right|} .
\end{gathered}
$$



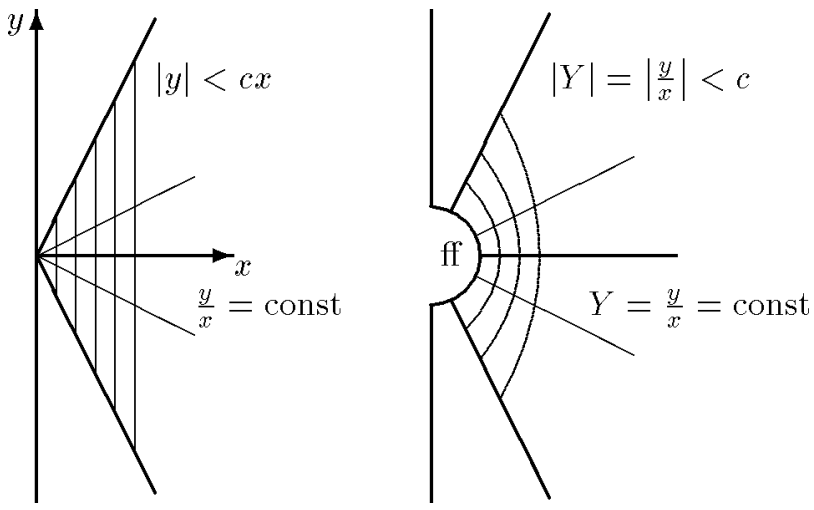

Fig. 2. The blowup of $C_{a}=\{x=0, y=0\}$; the $z$ coordinates are normal to the page and are not shown. The thin lines are the coordinate curves $Y=$ const and $x=$ const in the region $|Y|<c$ (which is disjoint from $\left.\beta\left[X ; C_{a}\right]^{*} \partial X\right)$, and their images under the blow-down map $\beta\left[X ; C_{a}\right]$.

Since in every region near the lift $\beta\left[X ; C_{a}\right]^{*} C_{a}$ of $C_{a}$ we can use one of these coordinate systems, and since away from there we can use coordinates as in (2.6) but with $w_{a}$ and $w^{a}$ interchanged, we have proved the following lemma.

Lemma 2.5. - Suppose that $X=\mathbb{S}_{+}^{n}$ and let $\beta=\beta\left[X ; C_{a}\right]$ be the blow-down map. Then the pull-back $\beta^{*}\left(\mathrm{RC}^{-1}\right)^{*} \pi^{a}$ of $\pi^{a}: \mathbb{R}^{n} \rightarrow X^{a}$ extends to a $\mathcal{C}^{\infty}$ map, which we also denote by $\pi^{a}$,

$$
\pi^{a}:\left[X ; C_{a}\right] \rightarrow \bar{X}^{a}
$$

Moreover, if $x^{a}$ is a boundary defining function on $\bar{X}^{a}\left(\right.$ e.g. $x^{a}=\left|w^{a}\right|^{-1}$ for $\left.\left|w^{a}\right|>1\right)$, then $\rho_{\partial X}=\left(\pi^{a}\right)^{*} x^{a}$ is a defining function for the lift of $\partial X$ to $\left[X ; C_{a}\right]$, i.e. for $\beta^{*} \partial X$.

COROLlary 2.6. - Suppose that $X=\mathbb{S}_{+}^{n}, f \in S_{\mathrm{phg}}^{r}\left(X^{a}\right)$. Then

$$
\left(\pi^{a}\right)^{*} f \in \rho_{\partial X}^{-r} \mathcal{C}^{\infty}\left(\left[X ; C_{a}\right]\right) .
$$

Here, following the previous lemma, we regard $\pi^{a}$ as the map in (2.11), and $\rho_{\partial X}$ is the defining function of $\beta\left[X ; C_{a}\right]^{*} \partial X$, i.e. of the lift of $\partial X$, and the subscript phg refers to classical (one-step polyhomogeneous) symbols (see Fig. 3).

This corollary shows that for a Euclidean many-body Hamiltonian, $H=\Delta+\sum_{a} V_{a}, V_{a}$ becomes a nice function on the compact resolved space $\left[\mathbb{S}_{+}^{n} ; C_{a}\right]$. Thus, to understand $H$, we need to blow up all the $C_{a}$. In order to analyze this iterated blow-up procedure, it is convenient to generalize the clean intersection properties to manifolds with corners $X$.

Let $X$ be a manifold with corners, and let $\mathcal{F}=\left\{F_{1}, \ldots, F_{N}\right\}$ be a collection of closed p-submanifolds of $X$. Following Melrose, [20, Chapter V], we say that $\mathcal{F}$ is a normal collection if for any point $p \in X$ there are local coordinates on a neighborhood $U$ of $p$ such that with some index sets $I_{l}, I_{l}^{\prime}, l=1, \ldots, N$,

$$
p \in F_{l} \Rightarrow F_{l} \cap U=\left\{x_{r}=0, r \in I_{l}, y_{s}=0, s \in I_{l}^{\prime}\right\} ;
$$

here the $x_{k}$ are defining functions of the boundary hypersurfaces through $p$. This simply means that there is a common product decomposition for all elements of the collection. 

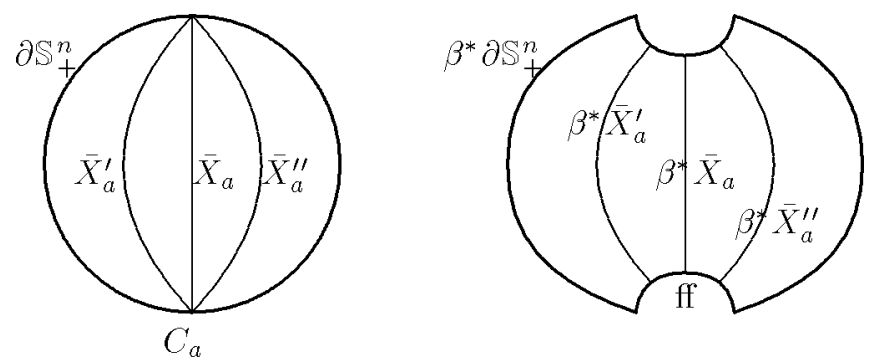

Fig. 3. The blowup of $C_{a}$ in $\mathbb{S}_{+}^{n} ; \beta=\beta\left[\mathbb{S}_{+}^{n} ; C_{a}\right]$ is the blow-down map and $\mathrm{ff}=\beta^{*} C_{a} . X_{a}^{\prime}$ and $X_{a}^{\prime \prime}$ denote translates of $X_{a}$ in $\mathbb{R}^{n}, \bar{X}_{a}^{\prime}=\operatorname{cl}\left(\operatorname{RC}\left(X_{a}^{\prime}\right)\right)$, etc. Note that the lifts of $\bar{X}_{a}, \bar{X}_{a}^{\prime}$ and $\bar{X}_{a}^{\prime \prime}$ become disjoint on $\left[\mathbb{S}_{+}^{n} ; C_{a}\right]$.

Using this definition, we can define cleanly intersecting families of submanifolds of $X$. Namely, given a finite family $\mathcal{F}$ of closed p-submanifolds $F_{i}$ of $X$ we say that $\mathcal{F}$ is a cleanly intersecting family if it is closed under intersection (in the sense that any two members are either disjoint, or their intersection is in the family) and for any $i$ and $j,\left\{F_{i}, F_{j}\right\}$ form a normal collection in the sense of Melrose [20, Chapter V]. As mentioned above, this means that there is a common product decomposition for any pair of elements of $\mathcal{F}$. In particular, if $X$ is a manifold without boundary, then this simply means that the $F_{i}$ pairwise intersect cleanly. Hence, $(X, \mathcal{C})$ is a many-body space if and only if $\mathcal{C}$ is a cleanly intersecting family in $\partial X$ which includes $\partial X$.

Just as in the case of a many-body space, inclusions give a partial order on a cleanly intersecting family $\mathcal{F}$. Thus, $F \in \mathcal{F}$ is minimal with respect to inclusion if there is no $F^{\prime} \in \mathcal{F}$ such that $F^{\prime} \neq F, F^{\prime} \subset F$. Since $\mathcal{F}$ is closed under intersection, this means exactly that for all $F^{\prime} \in \mathcal{F}$ either $F^{\prime}$ and $F$ are disjoint, or $F \subset F^{\prime}$.

LEMMA 2.7. - Let $\mathcal{F}$ be a cleanly intersecting family of p-submanifolds of $\partial X$. Suppose that $F \in \mathcal{F}$ is minimal with respect to inclusion. Then the lifted family, $\mathcal{F}^{\prime}$, consisting of the lifts of $F_{j}$, distinct from $F$, to $[X ; F]$, is also a cleanly intersecting family.

Proof. - We claim that for any $F_{i}, F_{j} \in \mathcal{F}$ the 4-tuple $\left\{F, F_{k}, F_{i}, F_{j}\right\}, F_{k}=F_{i} \cap F_{j}$, is a normal collection in the sense of Melrose. Indeed, this is clear if $F_{k}$ is disjoint from $F$; otherwise $F \subset F_{k}$ by our assumption.

So assume that $F \subset F_{k}$. By the normality of $\left\{F, F_{k}\right\}$, near any point $p$ in $F$ there are local coordinates $x_{r}, y_{s}$, on $X$ such that

$$
\begin{gathered}
F_{k}=\left\{x_{r}=0, r \in I_{k}^{\prime}, y_{s}=0, s \in I_{k}^{\prime \prime}\right\}, \\
F=\left\{x_{r}=0, r \in I^{\prime}, y_{s}=0, s \in I^{\prime \prime}\right\},
\end{gathered}
$$

and $I_{k}^{\prime} \subset I^{\prime}, I_{k}^{\prime \prime} \subset I^{\prime \prime}$. Similarly, by the normality of $\left\{F_{i}, F_{j}\right\}$ there are local coordinates $x_{r}^{\prime}, y_{s}^{\prime}$ near $p$ on $X$ such that

$$
\begin{aligned}
& F_{i}=\left\{x_{r}^{\prime}=0, r \in I_{i}^{\prime}, y_{s}^{\prime}=0, s \in I_{i}^{\prime \prime}\right\}, \\
& F_{j}=\left\{x_{r}^{\prime}=0, r \in I_{j}^{\prime}, y_{s}^{\prime}=0, s \in I_{j}^{\prime \prime}\right\},
\end{aligned}
$$

Thus,

$$
F_{k}=\left\{x_{r}^{\prime}=0, r \in I_{i}^{\prime} \cup I_{j}^{\prime}, y_{s}^{\prime}=0, s \in I_{i}^{\prime \prime} \cup I_{j}^{\prime \prime}\right\}
$$


Thus, the differentials of the coordinates $x_{r}^{\prime}, r \in I_{i}^{\prime} \cup I_{j}^{\prime}$, and $y_{s}^{\prime}, s \in I_{i}^{\prime \prime} \cup I_{j}^{\prime \prime}$, span the conormal bundle of $F_{k}$. The same holds for the differentials of $x_{r}, r \in I_{k}^{\prime}, y_{s}, s \in I_{k}^{\prime \prime}$. It follows that the differentials of $x_{r}^{\prime}, r \in I_{i}^{\prime} \cup I_{j}^{\prime}, x_{r}, r \notin I_{k}^{\prime}, y_{s}^{\prime}, s \in I_{i}^{\prime \prime} \cup I_{j}^{\prime \prime}, y_{s}, s \notin I_{k}^{\prime \prime}$ are independent at $F_{k}$ in a coordinate neighborhood of $p$, so these functions give local coordinates on $X$ near $p$ in terms of which $F, F_{k}, F_{i}$ and $F_{j}$ have common product decomposition: $F_{i}, F_{j}$ and $F_{k}$ given by (2.16)-(2.18), and $F$ by

$$
\begin{gathered}
F=\left\{x_{r}^{\prime}=0, r \in I_{i}^{\prime} \cup I_{j}^{\prime}, x_{r}=0, r \in I^{\prime} \backslash I_{k}^{\prime}, y_{s}^{\prime}=0, s \in I_{i}^{\prime \prime} \cup I_{j}^{\prime \prime},\right. \\
\left.y_{s}=0, s \in I^{\prime \prime} \backslash I_{k}^{\prime \prime}\right\} .
\end{gathered}
$$

This proves that $\left\{F, F_{k}, F_{i}, F_{j}\right\}$ is indeed a normal collection. Hence, by [20, Lemma V.11.2], it lifts to a normal collection of $\mathrm{p}$-submanifolds on $[X ; F]$. Writing $\beta$ for the blow-down map, and $\beta^{*} F_{k}$ for the lift of $F_{k}$, etc., we see in particular that $\left\{\beta^{*} F_{i}, \beta^{*} F_{j}\right\}$ is a normal collection whose intersection is $\beta^{*} F_{k}$ if $F_{k} \neq F$, and is empty otherwise. Putting together these facts we see that we have proved the lemma.

This lemma allows us to define $[X ; \mathcal{F}]$ if $\mathcal{F}$ is a cleanly intersecting family of p-submanifolds of $\partial X$. We do this by putting a total order on $\mathcal{F}$ which is compatible with the partial order given by inclusion. This can always be accomplished: pick a minimal element with respect to inclusion, and make it the minimal element of the total order. Proceeding inductively, if we already placed a total order on $\mathcal{F}^{\prime} \subset \mathcal{F}$, we choose any $F \in \mathcal{F} \backslash \mathcal{F}^{\prime}$ which is minimal with respect to inclusion in $\mathcal{F} \backslash \mathcal{F}^{\prime}$, and extend the total order to $\mathcal{F}^{\prime} \cup\{F\}$ by making $F$ the maximal element with respect to it. Having imposed a total order on $\mathcal{F}$ which is compatible with inclusion, we define $[X ; \mathcal{F}]$ to be the blow up $\left[X ; F_{1}, F_{2}, \ldots, F_{n}\right]$ where $\mathcal{F}=\left\{F_{1}, F_{2}, \ldots, F_{n}\right\}$ and $F_{1}<F_{2}<\cdots<F_{n}$, $<$ being the total order. Of course, a priori $[X ; \mathcal{F}]$ depends on the total order. The following lemma shows that this is not the case.

LEMMA 2.8. - If $\mathcal{F}$ is a cleanly intersecting family and $<,<$ are total orders on it which are compatible with inclusion, then the blow ups

$$
\begin{gathered}
{\left[X ; F_{1}, F_{2}, \ldots, F_{n}\right], \quad F_{1}<F_{2}<\cdots<F_{n},} \\
{\left[X ; F_{1}^{\prime}, F_{2}^{\prime}, \ldots, F_{n}^{\prime}\right], \quad F_{1}^{\prime}<^{\prime} F_{2}^{\prime}<^{\prime} \cdots<^{\prime} F_{n}^{\prime}}
\end{gathered}
$$

are canonically diffeomorphic.

Proof. - Since any total order compatible with inclusion can be obtained from any other one by repeatedly interchanging the order of adjacent elements, but keeping the order compatible with inclusion, it suffices to show that

$$
\left[X ; F_{1}, \ldots, F_{k}, F_{k+1}, \ldots, F_{n}\right] \text { and }\left[X ; F_{1}, \ldots, F_{k+1}, F_{k}, \ldots, F_{n}\right]
$$

are naturally isomorphic if both of these total orders respect inclusion. Now, either $F_{k} \cap F_{k+1}=\emptyset$, in which case the statement is clearly true, or $F_{k} \cap F_{k+1}=F_{j}$ for some $j$. Since inclusion is respected, we must have $j<k$. But upon the blow up of their intersection, any two closed psubmanifolds with normal intersection lift to be disjoint. Hence, on $\left[X ; F_{1}, \ldots, F_{k-1}\right]$ the lifts $\beta^{*} F_{k}$ and $\beta^{*} F_{k+1}$ are disjoint, and thus they can be blown up in either order. This proves the lemma.

Correspondingly, $[X ; \mathcal{F}]$ is defined independently of the total order used in the definition of the blown up space, assuming that it respects inclusion, so we can speak about $[X ; \mathcal{F}]$ without specifying such a total order. 
If $F_{i} \in \mathcal{F}$, we can always specify the total order so that every $F_{j} \in \mathcal{F}$ with $F_{j}<F_{i}$ satisfies $F_{j} \subset F_{i}$. Then the blow-up of $F_{i}$ commutes with all the ones preceding it. Hence, any function that is smooth on $\left[X ; F_{i}\right]$ pulls back to be smooth on $[X ; \mathcal{F}]$. Applying this in the Euclidean many-body setting we conclude that

Lemma 2.9. - Suppose that $X=\mathbb{S}_{+}^{n}$ and $\mathcal{X}$ is a linear family of subspaces of $\mathbb{R}^{n}$ as in the introduction. Then $V=\sum_{a} V_{a}, V_{a} \in S_{\mathrm{phg}}^{-m}\left(X^{a}\right)$, lifts to be an element of $\rho_{\partial X}^{m} \mathcal{C}^{\infty}([X ; \mathcal{C}])$ where $\rho_{\partial X}$ is the defining function of the lift of $C_{0}=\partial X$ under the blow-down map

$$
\beta_{\mathrm{Sc}}=\beta[X ; \mathcal{C}]:[X ; \mathcal{C}] \rightarrow X
$$

Our main interest is the study of differential operators, in particular the analysis of many-body Hamiltonians $H$. For this purpose we next investigate how vector fields lift under the blow up. First, we define $\mathcal{V}_{\mathrm{b}}(X ; \mathcal{F})$ as the Lie algebra of smooth vector fields on $X$ which are tangent to the boundary faces of $X$ and to each element of $\mathcal{F}$.

\section{LEMmA 2.10. - Each element of $\mathcal{V}_{\mathrm{b}}(X ; \mathcal{F})$ lifts to an element of $\mathcal{V}_{\mathrm{b}}([X ; \mathcal{F}])$.}

Proof. - It suffices to show that $V \in \mathcal{V}_{\mathrm{b}}(X ; \mathcal{F})$ lifts to be an element of $\mathcal{V}_{\mathrm{b}}\left([X ; F] ; \mathcal{F}^{\prime}\right)$ where $F$ is minimal with respect to inclusion and

$$
\mathcal{F}^{\prime}=\left\{\beta^{*} F^{\prime}: F^{\prime} \in \mathcal{F} \backslash\{F\}\right\} .
$$

Taking into account that for any $F^{\prime} \neq F,\left\{F, F^{\prime}\right\}$ is a normal collection of p-submanifolds of $X$, this claim follows from [20, Proposition V.11.1], or it can be checked directly by using projective coordinates on $[X ; F]$.

Remark 2.11. - It is not the case in general that $\mathcal{V}_{\mathrm{b}}(X ; \mathcal{F})$ lifts to span $\mathcal{V}_{\mathrm{b}}([X ; \mathcal{F}])$ over $\mathcal{C}^{\infty}([X ; \mathcal{F}])$. This statement is true, however, if $\mathcal{F}$ is a normal collection (i.e. all elements of $\mathcal{F}$ have product decomposition in the same coordinate system, not just pairs of elements), see [20, Proposition V.11.1].

We can now introduce the appropriate class of differential and pseudo-differential operators on many-body spaces $(X, \mathcal{C})$. These will include many-body Hamiltonians in the Euclidean setting as well as their resolvents (in the resolvent set).

First, we recall from [22] Melrose's definition of the Lie algebra of 'scattering vector fields' $\mathcal{V}_{\text {sc }}(X)$, defined for every manifold with boundary $X$. Before defining $\mathcal{V}_{\mathrm{sc}}(X)$, we recall that $\mathcal{V}_{\mathrm{b}}(X)$ denotes the set (in fact, Lie algebra) of smooth vector fields on $X$ which are tangent to $\partial X$. The algebra $\operatorname{Diff}_{\mathrm{b}}(X)$ of differential operators generated by $\mathcal{V}_{\mathrm{b}}(X)$ is called the algebra of $\mathrm{b}$-differential operators; $\operatorname{Diff}_{\mathrm{b}}^{k}(X)$ denotes the set of $\mathrm{b}$-differential operators of order (at most) $k$; here $b$ stands for 'boundary'. Then we define $\mathcal{V}_{\mathrm{sc}}(X)$ as

$$
\mathcal{V}_{\mathrm{sc}}(X)=x \mathcal{V}_{\mathrm{b}}(X)
$$

Note that this definition is intrinsic; for any other boundary defining function is a positive multiple of $x$, hence $\mathcal{V}_{\mathrm{sc}}(X)$ is independent of such choices. If $\left(x, y_{1}, \ldots, y_{n-1}\right)$ are coordinates on $X$ where $x$ is a boundary defining function, then locally a basis of $\mathcal{V}_{\mathrm{sc}}(X)$ is given by

$$
x^{2} \partial_{x}, \quad x \partial_{y_{j}}, \quad j=1, \ldots, n-1 .
$$


Correspondingly, there is a vector bundle ${ }^{\text {sc }} T X$ over $X$, called the scattering tangent bundle of $X$, such that $\mathcal{V}_{\mathrm{sc}}(X)$ is the set of all smooth sections of ${ }^{\mathrm{sc}} T X$ :

$$
\mathcal{V}_{\mathrm{sc}}(X)=\mathcal{C}^{\infty}\left(X ;{ }^{\mathrm{sc}} T X\right) .
$$

The dual bundle of ${ }^{\mathrm{sc}} T X$ (called the scattering cotangent bundle) is denoted by ${ }^{\mathrm{sc}} T^{*} X$. Thus, covectors $v \in{ }^{\mathrm{sc}} T_{p}^{*} X, p$ near $\partial X$, can be written as $v=\tau \frac{d x}{x^{2}}+\mu \cdot \frac{d y}{x}$. Hence, we have local coordinates $(x, y, \tau, \mu)$ on ${ }^{\mathrm{sc}} T^{*} X$ near $\partial X$. The scattering density bundle ${ }^{\mathrm{sc}} \Omega X$ is the density bundle associated to ${ }^{\mathrm{sc}} T^{*} X$, so locally near $\partial X$ it is spanned by $x^{-n-1} d x d y$ over $\mathcal{C}^{\infty}(X)$. Finally, Diff ${ }_{\mathrm{sc}}(X)$ is the algebra of differential operators generated by the vector fields in $\mathcal{V}_{\mathrm{sc}}(X) ; \operatorname{Diff}_{\mathrm{sc}}^{m}(X)$ stands for scattering differential operators of order (at most) $m$.

To establish the relationship between the scattering structure and the Euclidean scattering theory, we introduce local coordinates on $X$ near $p \in \partial X$ as above, and use these to identify the coordinate neighborhood $U$ of $p$ with a coordinate patch $U^{\prime}$ on the closed upper hemisphere $\mathbb{S}_{+}^{n}$ (which is just a closed ball) near its boundary. Such an identification preserves the scattering structure since this structure is completely natural. We further identify $\mathbb{S}_{+}^{n}$ with $\mathbb{R}^{n}$ via the radial compactification $\mathrm{RC}$ as in (2.3). The constant coefficent vector fields $\partial_{w_{j}}$ on $\mathbb{R}^{n}$ lift under RC to give a basis of ${ }^{\mathrm{sc}} T \mathbb{S}_{+}^{n}$. Thus, $V \in \mathcal{V}_{\mathrm{sc}}\left(\mathbb{S}_{+}^{n}\right)$ can be expressed as (ignoring the lifting in the notation)

$$
V=\sum_{j=1}^{n} a_{j} \partial_{w_{j}}, \quad a_{j} \in \mathcal{C}^{\infty}\left(\mathbb{S}_{+}^{n}\right) .
$$

As mentioned in the introduction, $a_{j} \in \mathcal{C}^{\infty}\left(\mathbb{S}_{+}^{n}\right)$ is equivalent to requiring that $\mathrm{RC}^{*} a_{j}$ is a classical (i.e. one-step polyhomogeneous) symbol of order 0 on $\mathbb{R}^{n}$. This description also shows that the positive Euclidean Laplacian, $\Delta$, is an element of $\operatorname{Diff}_{\mathrm{sc}}^{2}\left(\mathbb{S}_{+}^{n}\right)$, and that ${ }^{\mathrm{sc}} \Omega \mathbb{S}_{+}^{n}$ is spanned by the pull-back of the standard Euclidean density $|d w|$.

If $X$ is a manifold with boundary then any element of $\mathcal{V}_{\mathrm{sc}}(X)=x \mathcal{V}_{\mathrm{b}}(X)$ is automatically tangent to any submanifold $C$ of $\partial X$. Hence, due to Lemma 2.10, we can define the algebra of many-body differential operators as shown by the following proposition.

PROPOSITION 2.12. - If $(X, \mathcal{C})$ is a many-body space, then $\mathcal{V}_{\mathrm{sc}}(X)$ lifts to a subalgebra of $\mathcal{V}_{\mathrm{b}}([X ; \mathcal{C}])$. Correspondingly,

$$
\operatorname{Diff}_{\mathrm{Sc}}(X, \mathcal{C})=\mathcal{C}^{\infty}([X ; \mathcal{C}]) \otimes_{\mathcal{C}^{\infty}(X)} \operatorname{Diff}_{\mathrm{sc}}(X)
$$

is an algebra.

Proof. - By the first part of the statement, for any $V \in \mathcal{V}_{\mathrm{sc}}(X), f \in \mathcal{C}^{\infty}([X ; \mathcal{C}])$, the commutator $[V, f]=V f$ is in $\mathcal{C}^{\infty}([X ; \mathcal{C}])$.

In particular, since $\mathcal{V}_{\mathrm{sc}}(X)$ is the set of all smooth sections of ${ }^{\mathrm{sc}} T X$, its lift is the set of all smooth sections of the pull-back of the bundle ${ }^{\mathrm{sc}} T X \rightarrow X$ to $[X ; \mathcal{C}]$. This pull-back bundle will be denoted by ${ }^{\mathrm{Sc}} T[X ; \mathcal{C}]$. Its dual bundle is the pull-back of the bundle ${ }^{\mathrm{sc}} T^{*} X \rightarrow X$; it is denoted by ${ }^{\mathrm{Sc}} T^{*}[X ; \mathcal{C}]$.

Since in Euclidean many-body scattering $\Delta \in \operatorname{Diff}_{\mathrm{sc}}^{2}\left(\mathbb{S}_{+}^{n}\right)$ and $V=\sum_{a} V_{a} \in \mathcal{C}^{\infty}\left(\left[\mathbb{S}_{+}^{n} ; \mathcal{C}\right]\right)$, it follows immediately that $H=\Delta+V \in \operatorname{Diff}_{\mathrm{Sc}}^{2}\left(\mathbb{S}_{+}^{n}, \mathcal{C}\right)$.

\section{Many-body pseudo-differential calculus}

Let $(X, \mathcal{C})$ be a many-body space, and $\beta_{\mathrm{Sc}}:[X ; \mathcal{C}] \rightarrow X$ the blow-down map. There are two equivalent way of defining many-body pseudo-differential operators. We can either specify their

4 SÉRIE - TOME $34-2001-\mathrm{N}^{\circ} 3$ 
Schwartz kernels as conormal distributions on an appropriately resolved space, or we can define them as the quantization of certain symbols. Here we give both definitions and show their equivalence. We start with the kernel definition; readers who wish to start with the quantization definition should directly proceed to the paragraph of (3.8).

First, we recall the definition of Hörmander's conormal spaces $I^{m}$ in the context of manifolds with corners; their use originates from Melrose's work. A rather brief description of these conormal spaces appears in [22, Section 20]; a detailed discussion can be found in [20, Chapter VI]. Thus, suppose that $M$ is a manifold with corners, and $Y$ a closed embedded interior p-submanifold (i.e. product submanifold, discussed in the previous section); here 'interior' means that $Y$ is the closure of its intersection with the interior of $M$, i.e. that $Y$ is not a subset of any of the boundary hypersurfaces of $M$. Under this assumption, a neighborhood $U$ of $Y$ can be identified with a neighborhood of the zero section of the normal bundle of $Y$ in $M$, hence with the direct product of $Y$ with a neighborhood of the origin in $\mathbb{R}^{\operatorname{codim}(Y)}$. In particular, Hörmander's definition, [11, Section 2.4], can be applied: $u^{\prime} \in I_{c}^{m}(U, Y)$ means that $u^{\prime} \in \mathcal{C}_{c}^{-\infty}(U)$, and modulo $\mathcal{C}^{\infty}(U), u^{\prime}$ can be written as a finite sum of terms, each of which has the form

$$
(2 \pi)^{-(\operatorname{dim} M+2 \operatorname{codim} Y) / 4} \int_{\mathbb{R}^{\operatorname{codim}(Y)}} \mathrm{e}^{i \phi(z, \zeta)} a(z, \zeta) d \zeta,
$$

where $\phi$ is a linear phase function parametrizing $N^{*} Y$, and $\operatorname{supp} a \subset K \times \mathbb{R}^{\operatorname{codim}(Y)}$ for some $K$ compact, and satisfying symbol estimates

$$
\left|\left(D_{\zeta}^{\alpha} D_{z}^{\beta} a\right)(z, \zeta)\right| \leqslant C_{\alpha \beta}\langle\zeta\rangle^{m+(\operatorname{dim} M-2 \operatorname{codim} Y) / 4} .
$$

We remark that if we locally embed $M$ in a manifold without boundary, $\tilde{M}$, then $Y$ extends to a manifold without boundary $\tilde{Y}$, and then elements of $I_{c}^{m}(U, Y)$ are restrictions of elements of $I_{c}^{m}(\tilde{M}, \tilde{Y})$ to $M$, i.e. they are conormal distributions smoothly up to $\partial M$. Elements of $I^{m}(M, Y)$ are then distributions $u \in \mathcal{C}^{-\infty}(M) \cap \mathcal{C}^{\infty}(M \backslash Y)$ which become elements of $I_{c}^{m}(U, Y)$ for some neighborhood $U$ of $Y$ as above when multiplied by cut-off functions in $\mathcal{C}_{c}^{\infty}(U)$. Similarly, one-step polyhomogeneous (or classical) conormal distributions $u \in I_{\mathrm{os}}^{m}(M, Y)$ are those for which $a$ above can be chosen one-step polyhomogenenous with leading homogeneity $|\zeta|^{m+(\operatorname{dim} M-2 \operatorname{codim} Y) / 4}$.

If we do not require polyhomogeneous behavior at $Y$, there is no reason to require it at the boundary of $M$. We recall from [20, Chapter 4], see also [21], first that $\mathcal{A}^{l}(M)$ is the space of conormal functions on $M$, conormal to $\partial M$, which have weighted $L^{\infty}$ bounds, with a fixed weight, under application of $\operatorname{Diff}_{\mathrm{b}}(M)$, i.e.

$$
\mathcal{A}^{l}(M)=\left\{u \in \mathcal{C}^{-\infty}(M): \forall P \in \operatorname{Diff}_{\mathrm{b}}(M), P u \in \rho^{l} L^{\infty}(M)\right\} .
$$

Here $\rho$ is a total boundary defining function of $M$, i.e. it is the product of boundary defining functions of the boundary hypersurfaces of $M$. Note that elements of $\mathcal{A}^{l}(M)$ are smooth in the interior of $M$. Here we assigned the same order $l$ to every boundary hypersurface of $M$; in general the orders may be different on each hypersurface, and correspondingly we would have a real number associated to each hypersurface.

Next, $\mathcal{A}^{m, l}(M, Y)$ is the space of distributions $u \in \mathcal{C}^{-\infty}(M) \cap \mathcal{A}^{l}(M \backslash Y)$, which near $Y$ and modulo $\mathcal{A}^{l}(M)$ can be written as a finite sum of terms as in (3.1), with $a$ conormal, order $l$, to the boundary of $M$, and symbolic in $\zeta$, i.e. the estimates (3.2) hold after replacing $D_{z}^{\beta}$ by b-differential operators in $z$, and inserting factors $\rho^{l}$ in the right hand side. 
We can now proceed to defining many-body pseudo-differential operators by specifying what their Schwartz kernels are. Since they will map $\dot{\mathcal{C}}^{\infty}(X)$ to $\dot{\mathcal{C}}^{\infty}(X)$ continuously, hence in particular to $\mathcal{C}^{-\infty}(X)$, they have Schwartz kernels in $\mathcal{C}^{-\infty}(X \times X)$. To arrive at a reasonable description of which tempered distributions on $X^{2}=X \times X$ are allowed as Schwartz kernels, we first take an appropriate resolution of this space. For a thorough discussion of the resolution process in the geometric two-body scattering, see Melrose's paper [22, Appendix B], and in the three-body setting [40, Chapter 3].

First, the b-double space, $X_{\mathrm{b}}^{2}$, has been defined by Melrose as $\left[X^{2} ;(\partial X)^{2}\right]$. The front face of the blow-up is called the b-front face and is denoted by bf, while the lifts of the left and right boundary hypersurfaces of $X^{2}$, i.e. of $\partial X \times X$ and $X \times \partial X$ are denoted by lf and $\mathrm{rf}$ respectively. The diagonal $\Delta$ of $X^{2}$ lifts to a p-submanifold $\Delta_{\mathrm{b}}$ of $X_{\mathrm{b}}^{2}$ which intersects $\partial X_{\mathrm{b}}^{2}$ in the interior of the $b$-front face, bf. (The definition of p-submanifolds and the blow-up process were discussed at the beginning of the previous section.) Moreover, $\Delta_{\mathrm{b}}$ is naturally diffeomorphic to $X$. Hence, $\mathcal{C}$ can be regarded as a collection $\mathcal{C}^{\prime}$ of submanifolds of $\Delta_{\mathrm{b}}$, and, since $\Delta_{\mathrm{b}}$ is a p-submanifold of $X_{\mathrm{b}}^{2}$, these submanifolds form a cleanly intersecting family in $X_{\mathrm{b}}^{2}$. Therefore, the blow up

$$
X_{\mathrm{Sc}}^{2}=\left[X_{\mathrm{b}}^{2} ; \mathcal{C}^{\prime}\right]
$$

is well-defined by our previous results. Note that $\partial X \in \mathcal{C}$ by our assumption, so the definition includes the blow up of the lift of $\partial \Delta_{\mathrm{b}}$. It is easy to see that this space coincides with the $X_{\mathrm{Sc}}^{2}$ defined in [36] if $(X, \mathcal{C})$ is a 3-body space. We also introduce the notation $\mathrm{ff}_{C}$ for the lift of $C \in \mathcal{C}$, considered as a submanifold of $\Delta_{\mathrm{b}}$, to $X_{\mathrm{Sc}}^{2}$. Thus, the boundary hypersurfaces of $X_{\mathrm{Sc}}^{2}$ are $\beta^{*}$ lf, $\beta^{*} \mathrm{rf}, \beta^{*}$ bf, as well as $\mathrm{ff}_{C}, C \in \mathcal{C} ; \beta: X_{\mathrm{Sc}}^{2} \rightarrow X_{\mathrm{b}}^{2}$ is the blow-down map.

Noting that even $\mathcal{C}^{\prime} \cup\left\{\Delta_{\mathrm{b}}\right\}$ is a cleanly intersecting family, we conclude that $\Delta_{\mathrm{b}}$ lifts to a p-submanifold, $\Delta_{\mathrm{Sc}}$, of $X_{\mathrm{Sc}}^{2}$, which is disjoint from lf, rf and bf. Correspondingly, we define the set of many-body pseudo-differential operators by

$$
\Psi_{\mathrm{Scc}}^{m, l}(X, \mathcal{C})=\left\{\kappa \in \mathcal{A}^{m, l}\left(X_{\mathrm{Sc}}^{2}, \Delta_{\mathrm{Sc}} ;{ }^{\mathrm{sc}} \Omega_{R}\right): \kappa \equiv 0 \text { at } \beta^{*} \text { bf } \cup \beta^{*} \text { lf } \cup \beta^{*} \mathrm{rf}\right\} ;
$$

here ${ }^{\mathrm{sc}} \Omega_{R}$ is the pull-back of the scattering density bundle from the right factor and $\beta: X_{\mathrm{Sc}}^{2} \rightarrow X_{\mathrm{b}}^{2}$ is the blow-down map. With the notation of (3.3), by $\kappa \equiv 0$ we mean that the kernel, which is a polyhomogeneous function in $\mathcal{A}^{l}\left(X_{\mathrm{Sc}}^{2} \backslash \Delta_{\mathrm{Sc}}\right)$, hence in particular near $\beta^{*}$ lf, $\beta^{*}$ rf and $\beta^{*}$ bf, is rapidly decreasing at $\beta^{*}$ lf, $\beta^{*}$ rf and $\beta^{*}$ bf, i.e. it is in $\left(\rho_{\mathrm{lf}} \rho_{\mathrm{rf}} \rho_{\mathrm{bf}}\right)^{N} \rho^{l} L^{\infty}$ for all $N$, with similar conditions on the derivatives $P \kappa$, where $\rho_{\text {lf }}$ is a defining function of $\beta^{*}$ lf, etc.

Similarly we define the corresponding one-step polyhomogeneous ('classical') operators

$$
\Psi_{\mathrm{Sc}}^{m, l}(X, \mathcal{C})=\left\{\kappa \in \rho^{l} I_{\mathrm{os}}^{m}\left(X_{\mathrm{Sc}}^{2}, \Delta_{\mathrm{Sc}} ;{ }^{\mathrm{sc}} \Omega_{R}\right): \kappa \equiv 0 \text { at } \beta^{*} \text { bf } \cup \beta^{*} \text { lf } \cup \beta^{*} \mathrm{rf}\right\}
$$

where $\rho$ is the total boundary defining function of $X_{\mathrm{Sc}}^{2}$. In particular, conormal distributions of order $-\infty$ are smooth functions, so

$$
\Psi_{\mathrm{Sc}}^{-\infty, l}(X, \mathcal{C})=\left\{\kappa \in \rho^{l} \mathcal{C}^{\infty}\left(X_{\mathrm{Sc}}^{2}, \Delta_{\mathrm{Sc}} ;{ }^{\mathrm{sc}} \Omega_{R}\right): \kappa \equiv 0 \text { at } \beta^{*} \text { bf } \cup \beta^{*} \text { lf } \cup \beta^{*} \mathrm{rf}\right\},
$$

i.e. the kernels of operators in $\Psi_{\mathrm{Sc}}^{-\infty, l}(X, \mathcal{C})$ are smooth up to all boundary hypersurfaces of $X_{\mathrm{Sc}}^{2}$ (at least if $l$ is a non-negative integer), and vanish to infinite order at the lift of every boundary hypersurface of $X_{\mathrm{b}}^{2}$. Tensoring with vector bundles defines $\Psi_{\mathrm{Scc}}^{m, l}(X, \mathcal{C} ; E, F)$ and $\Psi_{\mathrm{Sc}}^{m, l}(X, \mathcal{C} ; E, F)$ for vector bundles $E$ and $F$ over $X$ as usual.

Since for all $F \in \mathcal{C}^{\prime}$ we have $F \subset \partial \Delta_{\mathrm{b}}$, we can do the blow up of $\partial \Delta_{\mathrm{b}} \in \mathcal{C}^{\prime}$ first, before blowing up other elements of $\mathcal{C}^{\prime}$ (normally we would do this blow up last by our total order 
construction). It follows that $X_{\mathrm{Sc}}^{2}$ is a blow up of the space $X_{\mathrm{sc}}^{2}=\left[X_{\mathrm{b}}^{2} ; \partial \Delta_{\mathrm{b}}\right]$. Hence, conormal distributions on $X_{\mathrm{sc}}^{2}$ pull back to be conormal on $X_{\mathrm{Sc}}^{2}$. Since the kernels of scattering pseudodifferential operators are conormal to $\Delta_{\mathrm{sc}}$ and to the boundary of $X_{\mathrm{sc}}^{2}$ with infinite order vanishing at every boundary face except the scattering front face, we conclude that these kernels pull back to $X_{\mathrm{Sc}}^{2}$ to be elements of the kernel space defined in (3.5), so $\Psi_{\mathrm{scc}}^{m, l}(X) \subset \Psi_{\mathrm{Scc}}^{m, l}(X, \mathcal{C})$.

Our alternative definition will proceed via localization and describing the kernels as quantizations of symbols in the local coordinate patches. Suppose now that $X=\mathbb{S}_{+}^{n}$ and $\mathcal{C}$ is a cleanly intersecting family of submanifolds of $\partial X=\partial \mathbb{S}_{+}^{n}=\mathbb{S}^{n-1}$. Here we do not assume that $\mathcal{C}$ arises from a family $\mathcal{X}$ of linear subspaces of $\mathbb{R}^{n}$. An equivalent definition of $\Psi_{\mathrm{Scc}}^{m, l}\left(\mathbb{S}_{+}^{n}, \mathcal{C}\right)$ is the following. Suppose that

$$
a \in \mathcal{A}^{-m, l}\left(\left[\mathbb{S}_{+}^{n} ; \mathcal{C}\right] \times \mathbb{S}_{+}^{n}\right)
$$

Here $-m$ is the order associated to the boundary hypersurface $\left[\mathbb{S}_{+}^{n} ; \mathcal{C}\right] \times \partial \mathbb{S}_{+}^{n}$, while $l$ is the order associated to the boundary hypersurfaces comprising $\left(\partial\left[\mathbb{S}_{+}^{n} ; \mathcal{C}\right]\right) \times \mathbb{S}_{+}^{n}$. We can unravel the definition (3.3) as follows. We identify $\operatorname{int}\left(\mathbb{S}_{+}^{n}\right)$ and $\operatorname{int}\left(\left[\mathbb{S}_{+}^{n} ; \mathcal{C}\right]\right)$ with $\mathbb{R}^{n}$ as usual (via $\mathrm{RC}^{-1}$ ); then for $a \in \mathcal{C}^{\infty}\left(\mathbb{R}_{w}^{n} \times \mathbb{R}_{\xi}^{n}\right)$ (3.8) is equivalent to the following property. (Recall first the definition of Diff ${ }_{\mathrm{b}}$ from the paragraph of (2.25).) For every $Q \in \operatorname{Diff}_{\mathrm{b}}^{k^{\prime}}\left(\left[\mathbb{S}_{+}^{n} ; \mathcal{C}\right]\right)$, acting on the first factor of $\mathbb{S}_{+}^{n}$ (i.e. in the $w$ variable), and $P \in \operatorname{Diff}_{\mathrm{b}}^{k}\left(\mathbb{S}_{+}^{n}\right)$, acting on the second factor of $\mathbb{S}_{+}^{n}$ (i.e. in the $\xi$ variable), $k, k^{\prime} \in \mathbb{N}$,

$$
P Q a \in \rho_{\infty}^{-m} \rho_{\partial}^{l} L^{\infty}\left(\mathbb{S}_{+}^{n} \times \mathbb{S}_{+}^{n}\right)
$$

where $\rho_{\infty}$ and $\rho_{\partial}$ are defining functions of the first and second factors of $\mathbb{S}_{+}^{n}$ respectively, so we can take $\rho_{\infty}=\langle\xi\rangle^{-1}, \rho_{\partial}=\langle w\rangle^{-1}$. Let $A=q_{L}(a)$ denote the left quantization of $a$ :

$$
A u(w)=(2 \pi)^{-n} \int \mathrm{e}^{i\left(w-w^{\prime}\right) \cdot \xi} a(w, \xi) u\left(w^{\prime}\right) d w^{\prime} d \xi
$$

understood as an oscillatory integral. Then $A \in \Psi_{\mathrm{Scc}}^{m, l}\left(\mathbb{S}_{+}^{n}, \mathcal{C}\right)$. Indeed, the kernel of $A$ is

$$
K\left(w, w^{\prime}\right)=\tilde{a}\left(w, w-w^{\prime}\right)
$$

where $\tilde{a}$ is the inverse Fourier transform of $a$ in the $\xi$ variable, i.e. $\tilde{a}=\mathcal{F}_{\xi}^{-1} a$. Thus, $\tilde{a}(w, W)$ is smooth away from $W=0$, is conormal to $W=0$, and it is rapidly decreasing with all derivatives in $W$. More precisely, the rapid decay means that for all $k$ and $Q \in \operatorname{Diff}_{\mathrm{b}}\left(\left[\mathbb{S}_{+}^{n} ; \mathcal{C}\right]\right)$ and for all $\alpha$,

$$
\sup _{|W| \geqslant 1, w \in \mathbb{R}^{n}}\left(|w|^{l}|W|^{k}\left|Q_{w} D_{W}^{\alpha} \tilde{a}(w, W)\right|\right)<\infty
$$

Taking into account the geometry of $X_{\mathrm{Sc}}^{2}$, in particular that $\left|w-w^{\prime}\right|^{-1}$ vanishes at all faces of the blow-up (3.4) but the front faces (i.e. it vanishes at $\beta^{*}$ lf, $\beta^{*}$ rf and $\beta^{*}$ bf), we see that $K$ vanishes to infinite order at these faces. Similar arguments describe the behavior of $K$ near $\Delta_{\mathrm{Sc}}$, proving that $A \in \Psi_{\mathrm{Scc}}^{m, l}\left(\mathbb{S}_{+}^{n}, \mathcal{C}\right)$.

Conversely, if $A \in \Psi_{\mathrm{Scc}}^{m, l}\left(\mathbb{S}_{+}^{n}, \mathcal{C}\right)$ then there exists a satisfying (3.9) such that $A=q_{L}(a)$. Namely, we let $\tilde{a}(w, W)=K(w, w-W)$ and let $a$ be the Fourier transform of $\tilde{a}$ in $W$. The conormal estimates for $K$ (hence for $\tilde{a}$ ) give the symbolic estimates (3.9) for $a$. 
Similar conclusions hold for the right quantization $B=q_{R}(b)$ of a symbol $b$ :

$$
B u(w)=(2 \pi)^{-n} \int \mathrm{e}^{i\left(w-w^{\prime}\right) \cdot \xi} b\left(w^{\prime}, \xi\right) u\left(w^{\prime}\right) d w^{\prime} d \xi
$$

In addition, the polyhomogeneous class $\Psi_{\mathrm{Sc}}^{m, l}\left(\mathbb{S}_{+}^{n}, \mathcal{C}\right)$ is given by the quantization of symbols

$$
a \in \rho_{\infty}^{-m} \rho_{\partial}^{l} \mathcal{C}^{\infty}\left(\left[\mathbb{S}_{+}^{n} ; \mathcal{C}\right] \times \mathbb{S}_{+}^{n}\right)
$$

Since differential operators $\sum a_{\alpha}(w) D^{\alpha}$ are just the left quantization of the symbols $a(w, \xi)=$ $\sum a_{\alpha}(w) \xi^{\alpha}$, it follows immediately that

$$
\operatorname{Diff}_{\mathrm{Sc}}^{m}(X, \mathcal{C}) \subset \Psi_{\mathrm{Sc}}^{m}(X, \mathcal{C})
$$

This conclusion also follows directly from the description of the kernels since the kernel of a differential operator is a differentiated delta-distribution associated to the diagonal.

Note that, as usual, one can allow symbols $a$ depending on $w, w^{\prime}$ and $\xi$, so e.g. if $a \in \rho_{\infty}^{-m} \rho_{\partial, L}^{l} \rho_{\partial, R}^{l^{\prime}} \mathcal{C}^{\infty}\left(\left[\mathbb{S}_{+}^{n} ; \mathcal{C}\right] \times\left[\mathbb{S}_{+}^{n} ; \mathcal{C}\right] \times \mathbb{S}_{+}^{n}\right), \rho_{\partial, L}$ and $\rho_{\partial, R}$ denoting total boundary defining functions of the first and second factor of $\left[\mathbb{S}_{+}^{n} ; \mathcal{C}\right]$ respectively (i.e. they are pull-backs of a boundary defining function of $\mathbb{S}_{+}^{n}$ ), then

$$
A u(w)=(2 \pi)^{-n} \int \mathrm{e}^{i\left(w-w^{\prime}\right) \cdot \xi} a\left(w, w^{\prime}, \xi\right) u\left(w^{\prime}\right) d w^{\prime} d \xi
$$

defines an operator $A \in \Psi_{\mathrm{Sc}}^{m, l+l^{\prime}}\left(\mathbb{S}_{+}^{n}, \mathcal{C}\right)$.

This characterization allows the application of the standard tools of the theory of pseudodifferential operators. In particular, if $A \in \Psi_{\mathrm{Sc}}^{m, l}(X, \mathcal{C})$ is written as the left quantization of a symbol $a$ and $B \in \Psi_{\mathrm{Sc}}^{m^{\prime}, l^{\prime}}(X, \mathcal{C})$ is written as the right quantization of a symbol $b$, so

$$
a \in \rho_{\infty}^{-m} \rho_{\partial}^{l} \mathcal{C}^{\infty}\left(\left[\mathbb{S}_{+}^{n} ; \mathcal{C}\right] \times \mathbb{S}_{+}^{n}\right), \quad b \in \rho_{\infty}^{-m^{\prime}} \rho_{\partial}^{l^{\prime}} \mathcal{C}{ }^{\infty}\left(\left[\mathbb{S}_{+}^{n} ; \mathcal{C}\right] \times \mathbb{S}_{+}^{n}\right)
$$

then the operator $A B$ is given by

$$
A B u(w)=(2 \pi)^{-n} \int \mathrm{e}^{i\left(w-w^{\prime}\right) \cdot \xi} a(w, \xi) b\left(w^{\prime}, \xi\right) u\left(w^{\prime}\right) d w^{\prime} d \xi
$$

Here $c\left(w, w^{\prime}, \xi\right)=a(w, \xi) b\left(w^{\prime}, \xi\right)$ is in $\rho_{\infty}^{-m-m^{\prime}} \rho_{\partial, L}^{l} \rho_{\partial, R}^{l^{\prime}} \mathcal{C}^{\infty}\left(\left[\mathbb{S}_{+}^{n} ; \mathcal{C}\right] \times\left[\mathbb{S}_{+}^{n} ; \mathcal{C}\right] \times \mathbb{S}_{+}^{n}\right)$, so we conclude that $A B \in \Psi_{\mathrm{Sc}}^{m+m^{\prime}, l+l^{\prime}}(X, \mathcal{C})$. In addition, the adjoint $A^{*}$ of $A$ is the right quantization of $\bar{a}$, so $A^{*} \in \Psi_{\mathrm{Sc}}^{m, l}(X, \mathcal{C})$. Analogously, $\Psi_{\mathrm{Scc}}\left(\mathbb{S}_{+}^{n}, \mathcal{C}\right)$ is also closed under composition and adjoints. These statements can be seen also from the standard more explicit formulae. For example, if $B$ is the left quantization of a symbol $b^{\prime}$, the composition formula, including the remainder terms, only involves derivatives of the form $D_{\xi}^{\alpha} D_{w}^{\alpha} b^{\prime}$, and $D_{w}^{\alpha} \in \operatorname{Diff}_{\mathrm{sc}}^{|\alpha|}\left(\mathbb{S}_{+}^{n}\right) \subset$ $\operatorname{Diff}_{\mathrm{b}}^{|\alpha|}\left(\left[\mathbb{S}_{+}^{n} ; \mathcal{C}\right]\right)$, so we see that $\Psi_{\mathrm{Scc}}\left(\mathbb{S}_{+}^{n}, \mathcal{C}\right)$ is closed under composition.

This discussion can be carried over to arbitrary manifolds with boundary $X$ by locally identifying $X$ with $\mathbb{S}_{+}^{n}$ and using that our arguments are local in $\mathbb{S}_{+}^{n}$. More precisely, suppose that $\left\{U_{1}, \ldots, U_{k}\right\}$ is an open cover of $X$ by coordinate patches, and identify each $U_{i}$ with a coordinate patch $U_{i}^{\prime}$ of $\mathbb{S}_{+}^{n}$. We write $\phi_{i}: U_{i} \rightarrow U_{i}^{\prime}$ for the identification. Let $\mathcal{C}_{i}^{\prime}$ denote the family given by the image of elements of $\mathcal{C}$ in $U_{i}^{\prime}$. Then $A \in \Psi_{\mathrm{Sc}}^{m, l}(X, \mathcal{C})$ if and only if there exist 
operators $A_{i}^{\prime} \in \Psi_{\mathrm{Sc}}^{m, l}\left(\mathbb{S}_{+}^{n} ; \mathcal{C}^{\prime}\right)$ with kernel supported in the inverse image of $U_{i}^{\prime} \times U_{i}^{\prime}$ in $\left(\mathbb{S}_{+}^{n}\right)_{\mathrm{Sc}}^{2}$ and $R \in \dot{\mathcal{C}}^{\infty}\left(X \times X ;{ }^{\mathrm{sc}} \Omega_{R}\right)$ such that

$$
A=\sum_{i}\left(\phi_{i}^{*} A_{i}^{\prime}\left(\phi_{i}^{-1}\right)^{*}\right)+R
$$

Note that the support condition on $A_{i}^{\prime}$ ensures that this expression makes sense. To see this, just introduce a partition of unity $\rho_{i} \in \mathcal{C}^{\infty}(X)$ subordinate to the cover, and let $\psi_{i} \in \mathcal{C}^{\infty}(X)$ be identically 1 in a neighborhood of $\operatorname{supp} \rho_{i}$. Then

$$
A=\sum_{i} A \rho_{i}=\sum_{i} \psi_{i} A \rho_{i}+\sum_{i}\left(1-\psi_{i}\right) A \rho_{i}
$$

It is straightforward to check directly from the definition of $\Psi_{\mathrm{Sc}}^{m, l}(X, \mathcal{C})$ that the last term is given by a kernel in $\dot{\mathcal{C}}^{\infty}\left(X \times X ;{ }^{\mathrm{sc}} \Omega_{R}\right)$, while $A_{i}^{\prime}=\left(\phi_{i}^{-1}\right)^{*} \psi_{i} A \rho_{i} \phi_{i}^{*} \in \Psi_{\mathrm{Sc}}^{m, l}\left(\mathbb{S}_{+}^{n}, \mathcal{C}_{i}^{\prime}\right)$ with the claimed support properties. Thus, our results for $\Psi_{\mathrm{Sc}}^{m, l}\left(\mathbb{S}_{+}^{n}, \mathcal{C}\right)$ immediately show the following theorem.

THEOREM 3.1. - Both $\Psi_{\mathrm{Sc}}(X, \mathcal{C})$ and $\Psi_{\mathrm{Scc}}(X, \mathcal{C})$ are $*$-algebras (with respect to composition and taking adjoints).

Since $\Psi_{\mathrm{Scc}}^{m, 0}\left(\mathbb{S}_{+}^{n}, \mathcal{C}\right) \subset \Psi_{\infty}^{m}\left(\mathbb{R}^{n}\right)$, where $\Psi_{\infty}^{m}\left(\mathbb{R}^{n}\right)$ is the class of pseudo-differential operators defined by Hörmander [12, Section 18.1], arising by a quantization of symbols $a \in \mathcal{C}^{\infty}\left(\mathbb{R}^{n} \times \mathbb{R}^{n}\right)$ satisfying

$$
\left|D_{w}^{\alpha} D_{\xi}^{\beta} a(w, \xi)\right| \leqslant C_{\alpha \beta}\langle\xi\rangle^{m-|\beta|}
$$

and

$$
\Psi_{\infty}^{m}\left(\mathbb{R}^{n}\right):\langle w\rangle^{-s} H^{r}\left(\mathbb{R}^{n}\right) \rightarrow\langle w\rangle^{-s} H^{r-m}\left(\mathbb{R}^{n}\right),
$$

we immediately deduce the boundedness of elements of $\Psi_{\mathrm{Scc}}^{m, l}(X, \mathcal{C})$ between the appropriate weighted Sobolev spaces.

THEOREM 3.2. - If $A \in \Psi_{\mathrm{Scc}}^{m, l}(X, \mathcal{C})$ then $A: H_{\mathrm{sc}}^{r, s}(X) \rightarrow H_{\mathrm{sc}}^{r-m, s+l}(X)$ is bounded.

There is another way of characterizing the calculus $\Psi_{\mathrm{Scc}}^{m, l}\left(\mathbb{S}_{+}^{n}, \mathcal{C}\right)$ via Hörmander's Weyl calculus (see [12, Section 18.5]). We describe it briefly here, only considering the Euclidean setting where the $C_{a}$ arise from linear subspaces $X_{a}$; it is straightforward to check that it agrees with the definition we have given above in terms of quantization of symbols as in (3.8). Namely, $\Psi_{\mathrm{Scc}}^{\infty,-\infty}\left(\mathbb{S}_{+}^{n}\right)$ is just the calculus on $\mathbb{R}^{n}$ arising from the metric

$$
g^{(0)}=\frac{d w^{2}}{\langle w\rangle^{2}}+\frac{d \xi^{2}}{\langle\xi\rangle^{2}} .
$$

Similarly, if we take $\mathcal{C}^{\prime}$ to consist of a single element $C_{a}, a \neq 0$, and if $\left(w_{a}, w^{a}\right)$ is the usual splitting of the coordinates, then $\Psi_{\mathrm{Scc}}^{\infty,-\infty}\left(\mathbb{S}_{+}^{n}, \mathcal{C}^{\prime}\right)$ arises from the metric

$$
g^{(a)}=\frac{d w_{a}^{2}}{\langle w\rangle^{2}}+\frac{\left(d w^{a}\right)^{2}}{\left\langle w^{a}\right\rangle^{2}}+\frac{d \xi^{2}}{\langle\xi\rangle^{2}} .
$$

In the three-body problem, $C_{a} \cap C_{b}=\emptyset$ if $a, b \neq 0$, we define the metric by localizing the $g^{(a)}$, i.e. we consider a partition of unity $\phi_{a} \in \mathcal{C}^{\infty}\left(\mathbb{S}_{+}^{n}\right), a \in I$, $\operatorname{supp} \phi_{a} \cap C_{b}=\emptyset$ unless $b=0$, and 
define the metric

$$
g=\sum_{a} \phi_{a} g^{(a)} .
$$

(Here the $\phi_{a}$ are pulled back to the cotangent bundle by the bundle projection.) Since the $g^{(a)}$ are equivalent near $C_{0}^{\prime}$, it follows that $g$ is indeed slowly varying. Note that if $\phi_{a}$ is supported close to $C_{a}$, which we can arrange by enlarging the support of $\phi_{0}, d w_{a}^{2} /\langle w\rangle^{2}$ above can be replaced by $d w_{a}^{2} /\left\langle w_{a}\right\rangle^{2}$.

In general we simply repeat this procedure. Thus, to define the appropriate metric on $T^{*} X^{c}$ if it has been defined on $T^{*} X^{a}$ for every $a$ with $X^{a} \subset X^{c}$, we define a partition of unity $\phi_{a} \in$ $\mathcal{C}^{\infty}\left(\bar{X}^{c}\right)$ with $\operatorname{supp} \phi_{a} \cap C_{b}^{c}=\emptyset$ unless $C_{a}^{c} \subsetneq C_{b}^{c}$. Here $X^{c}=X^{a} \oplus X_{a}^{c}$ and $\left.C_{a}^{c}=\partial \bar{X}^{c} \cap \operatorname{cl}\left(X_{a}^{c}\right)\right)$. We extend the metric $g^{a}$ on $T^{*} X^{a}$ to a symmetric 2-cotensor on $T^{*} X^{c}$ using the orthogonal decomposition $X^{c}=X^{a} \oplus X_{a}^{c}$, and let

$$
g^{(a)}=g^{a}+\frac{\left(d w_{a}^{c}\right)^{2}}{\left\langle w_{a}^{c}\right\rangle^{2}}+\frac{\left(d \xi_{a}^{c}\right)^{2}}{\left\langle\xi^{c}\right\rangle^{2}} .
$$

Then

$$
g^{c}=\sum_{a: X^{a} \subset X^{c}} \phi_{a} g^{(a)}
$$

gives the desired metric on $T^{*} X^{c}$. The weights corresponding to elements of $\Psi_{\mathrm{Scc}}^{m, l}\left(\mathbb{S}_{+}^{n}, \mathcal{C}\right)$ then are $\langle\xi\rangle^{m}\langle w\rangle^{-l}$, i.e. the condition on the 'amplitude' $a$ is $a \in S\left(\langle\xi\rangle^{m}\langle w\rangle^{-l}, g^{0}\right)$.

After this brief discussion of the relationship of $\Psi_{\mathrm{Sc}}^{*, *}\left(\mathbb{S}_{+}^{n}, \mathcal{C}\right)$ with Hörmander's Weyl calculus, we return to the general setting to describe the principal symbol map and its analog at $\partial X$.

\section{The principal symbol and the indicial operators}

Since the inclusion of $H_{\mathrm{sc}}^{r^{\prime}} s^{\prime}(X)$ to $H_{\mathrm{sc}}^{r, s}(X)$ is compact for $r^{\prime}>r, s^{\prime}>s$, it suffices to understand $A \in \Psi_{\mathrm{Scc}}^{m, l}(X, \mathcal{C})$ modulo $\Psi_{\mathrm{Scc}}^{m-1, l+1}(X, \mathcal{C})$ to analyze its spectral properties. Now, Hörmander's principal symbol map on $\Psi_{\infty}^{m}\left(\mathbb{R}^{n}\right)$ restricts to a principal symbol map

$$
\sigma_{\mathrm{Sc}, m}: \Psi_{\mathrm{Sc}}^{m, 0}\left(\mathbb{S}_{+}^{n}, \mathcal{C}\right) \rightarrow S_{h}^{m}\left({ }^{\mathrm{Sc}} T^{*}\left[\mathbb{S}_{+}^{n} ; \mathcal{C}\right]\right)
$$

$S_{h}\left({ }^{\mathrm{Sc}} T^{*}\left[\mathbb{S}_{+}^{n} ; \mathcal{C}\right]\right)$ denoting the space of smooth symbols which are homogeneous of degree $m$. Due to its invariance and its local nature, it immediately extends to a map

$$
\sigma_{\mathrm{Sc}, m}: \Psi_{\mathrm{Sc}}^{m, 0}(X, \mathcal{C}) \rightarrow S_{h}^{m}\left({ }^{\mathrm{Sc}} T^{*}[X ; \mathcal{C}]\right) .
$$

We radially compactify the fibers of ${ }^{\mathrm{Sc}} T^{*}[X ; \mathcal{C}]$ (i.e. replace the vector spaces by balls) and let ${ }^{\mathrm{Sc}} S^{*}[X ; \mathcal{C}]$ be the new boundary face (i.e. the boundary of ${ }^{\mathrm{Sc}} T^{*}[X ; \mathcal{C}]$ at fiber-infinity). This allows us to write $\sigma_{\mathrm{Sc}, m}$ as a map

$$
\sigma_{\mathrm{Sc}, m}: \Psi_{\mathrm{Sc}}^{m, 0}(X, \mathcal{C}) \rightarrow \mathcal{C}^{\infty}\left({ }^{\mathrm{Sc}} S^{*}[X ; \mathcal{C}] ;\left(N^{* \mathrm{Sc}} S^{*}[X ; \mathcal{C}]\right)^{-m}\right)
$$

The line bundle $N^{* \mathrm{Sc}} S^{*}[X ; \mathcal{C}]$ is locally spanned by the pull-back of $d\left(|\xi|^{-1}\right)$ from ${ }^{\mathrm{Sc}} T^{*}[X ; \mathcal{C}]$, so (4.3) is obtained from (4.1) by writing homogeneous functions $a(w, \xi)$ of degree $m$ as 
$a_{0}(w, \hat{\xi})|\xi|^{m}, \hat{\xi}=\xi /|\xi|$, considering $a_{0}$ as a function on the cosphere bundle, and using $N^{* \operatorname{Sc}} S^{*}[X ; \mathcal{C}]$ to take care of the factor $|\xi|^{m}$ invariantly. We then have a short exact sequence

$$
0 \rightarrow \Psi_{\mathrm{Sc}}^{m-1,0}(X, \mathcal{C}) \rightarrow \Psi_{\mathrm{Sc}}^{m, 0}(X, \mathcal{C}) \rightarrow \mathcal{C}^{\infty}\left({ }^{\mathrm{Sc}} S^{*}[X ; \mathcal{C}] ;\left(N^{* \mathrm{Sc}} S^{*}[X ; \mathcal{C}]\right)^{-m}\right) \rightarrow 0
$$

as usual.

An operator $A \in \Psi_{\mathrm{Sc}}^{m, 0}(X, \mathcal{C})$ is certainly determined modulo $\Psi_{\mathrm{Sc}}^{m, 1}(X, \mathcal{C})$ by the restriction of its kernel to the front faces $\mathrm{ff}_{C}, C \in \mathcal{C}$, of the blow up (3.4) - by restriction we really mean the restriction of the kernel as a section of the pull-back of the density bundle ${ }^{\mathrm{sc}} \Omega_{R}$ from $X_{\mathrm{Sc}}^{2}$ to $\mathrm{ff}_{C}$ (we keep denoting this bundle by ${ }^{\mathrm{sc}} \Omega_{R}$ ). Note that ${ }^{\mathrm{sc}} \Omega_{R}$ is locally spanned by $\left|d w^{\prime}\right|$, so locally this amounts to factoring out $\left|d w^{\prime}\right|$ from the distributional density kernel $K\left(w, w^{\prime}\right)\left|d w^{\prime}\right|=\tilde{a}\left(w, w-w^{\prime}\right)\left|d w^{\prime}\right|$ from the kernel of $A$ (with the notation of (3.11)), and restricting $\tilde{a}$ (i.e. $K$ ) to the boundary of $\left[\mathbb{S}_{+}^{n} ; \mathcal{C}\right]_{w} \times \mathbb{R}_{W}^{n}, W=w-w^{\prime}$.

Thus, we define the normal operator $N_{C}(A)$ of $A$ at $C \in \mathcal{C}$ to be the restriction of the kernel of $A$ to $\mathrm{ff}_{C}$, which, as we recall, is the front face resulting when $C$, identified as a submanifold $C^{\prime}$ of $\Delta_{\mathrm{b}}$, is blown up in (3.4). Since $\Delta_{\mathrm{Sc}}$ intersects this face transversally, the result is a conormal distribution, conormal to the intersection of $\Delta_{\mathrm{Sc}}$ with this front face, which decays rapidly at $\mathrm{ff}_{C} \cap$ bf, i.e. with respect to $W$ with the above notation. We let

$$
\begin{aligned}
& \dot{I}_{\mathrm{os}, \mathrm{bf}}^{m^{\prime}}\left(\mathrm{ff}_{C}, \Delta_{\mathrm{Sc}} \cap \mathrm{ff}_{C}\right) \\
& \quad=\left\{\kappa \in I_{\mathrm{os}}^{m^{\prime}}\left(\mathrm{ff}_{C}, \Delta_{\mathrm{Sc}} \cap \mathrm{ff}_{C}\right): \kappa \text { vanishes to infinite order at bf } \cap \mathrm{ff}_{C}\right\} .
\end{aligned}
$$

Thus, for each $C \in \mathcal{C}$,

$$
N_{C}: \Psi_{\mathrm{Sc}}^{m, 0}(X, \mathcal{C}) \mapsto \dot{I}_{\mathrm{os}, \mathrm{bf}}^{m-1 / 4}\left(\mathrm{ff}_{C}, \Delta_{\mathrm{Sc}} \cap \mathrm{ff}_{C} ;{ }^{\mathrm{sc}} \Omega_{R}\right) ;
$$

the new order $m-1 / 4$ is due to the change of the dimension of the total space in the defining equation (3.1), the order of the amplitude $a$ as a symbol being unchanged.

There are compatibilty conditions between the normal operators $N_{C}, C \in \mathcal{C}$, and the principal symbol map $\sigma_{\mathrm{Sc}, m}$. Namely, the principal symbol of the conormal singularity of $N_{C}$ at $\Delta_{\mathrm{Sc}}$ must be the same as the restriction of $\sigma_{\mathrm{Sc}}$ to the front face, i.e.

$$
\sigma_{m-1 / 4}\left(N_{C}(A)\right)=\left.\sigma_{\mathrm{Sc}, m}(A)\right|_{\operatorname{sc}_{\mathrm{ff}_{C}}^{*}[X ; \mathcal{C}]} .
$$

(Note that with the density factor ${ }^{\mathrm{sc}} \Omega_{R}$ this is indeed invariantly defined.) In addition, if $C_{b} \cap C_{c} \neq \emptyset$, then $N_{C_{b}}$ and $N_{C_{c}}$ must be equal on the intersection $\mathrm{ff}_{C_{b}} \cap \mathrm{ff}_{C_{c}}$, i.e.

$$
\left.N_{C_{c}}(A)\right|_{\mathrm{ff}_{C_{b}} \cap \mathrm{ff}_{C_{c}}}=\left.N_{C_{b}}(A)\right|_{\mathrm{ff}_{C_{b}} \cap \mathrm{ff}_{C_{c}}} .
$$

(Note that the diagonal $\Delta_{\mathrm{Sc}}$ is transversal to $\mathrm{ff}_{C_{b}} \cap \mathrm{ff}_{C_{c}}$, hence one can restrict distributions which are conormal to $\Delta_{\mathrm{Sc}}$, to $\mathrm{ff}_{C_{b}} \cap \mathrm{ff}_{C_{c}}$.) We denote by $\mathcal{N}_{m}$ the subspace of the joint target space of these maps which satisfy these matching conditions:

$$
\begin{gathered}
\mathcal{N}_{m}=\left\{\left(a,\left\{K_{b}: b \in I\right\}\right) \in \mathcal{C}^{\infty}\left({ }^{\mathrm{Sc}} S^{*}[X ; \mathcal{C}]\right) \times \prod_{b \in I} \dot{I}_{\mathrm{bf}}^{m-1 / 4}\left(\mathrm{ff}_{C_{b}}, \Delta_{\mathrm{Sc}} \cap \mathrm{ff}_{C_{b}} ;{ }^{\mathrm{sc}} \Omega_{R}\right):\right. \\
\forall b \in I, a=\sigma_{m-1 / 4}\left(\left.K_{b}\right|_{\mathrm{sc}} S_{\mathrm{ff}_{C}}^{*}[X ; \mathcal{C}]\right) \\
\forall b, c \in I,\left.K_{c}\right|_{\left.\mathrm{ff}_{C_{b}} \cap \mathrm{ff}_{C_{c}}=\left.K_{b}\right|_{\mathrm{ff}_{C_{b}}} \cap \mathrm{ff}_{C_{c}}\right\} .}
\end{gathered}
$$


In the special case of $m=-\infty$, the kernels are smooth functions on $X_{\mathrm{Sc}}^{2}$, and we define

$$
\begin{aligned}
& \mathcal{N}_{-\infty}=\{\left\{K_{b}: b \in I\right\} \in \prod_{b \in I} \mathcal{C}_{\mathrm{bf}}^{\infty}\left(\mathrm{ff}_{C_{b}} ;{ }^{\mathrm{sc}} \Omega_{R}\right): \\
& \forall b, c \in I,\left.K_{c}\right|_{\mathrm{ff}_{C_{b}} \cap \mathrm{ff}_{C_{c}}}=\left.K_{b}\right|_{\left.\mathrm{ff}_{C_{b}} \cap \mathrm{ff}_{C_{c}}\right\}} ;
\end{aligned}
$$

here $\dot{\mathcal{C}}_{\mathrm{bf}}^{\infty}\left(\mathrm{ff}_{C_{b}}\right)$ denotes the space of smooth functions on $\mathrm{ff}_{C_{b}}$ which vanish with all derivatives at bf.

On the other hand, specifying any element of $\mathcal{N}_{m}$, i.e. any principal symbol as well as normal operators satisfying the matching conditions (4.7)-(4.8), one can find a conormal distribution on $X_{\mathrm{Sc}}^{2}$ with precisely these principal symbol and normal operators since $X_{\mathrm{Sc}}^{2}$ is a manifold with corners and $\Delta_{\mathrm{Sc}}$ is a p-submanifold. Thus, the combined principal symbol and normal operator maps give rise to a short exact sequence

$$
0 \rightarrow \Psi_{\mathrm{Sc}}^{m-1,1}(X, \mathcal{C}) \rightarrow \Psi_{\mathrm{Sc}}^{m, 0}(X, \mathcal{C}) \rightarrow \mathcal{N}_{m} \rightarrow 0 .
$$

In case $m=-\infty$, this changes to the analogous short exact sequence

$$
0 \rightarrow \Psi_{\mathrm{Sc}}^{-\infty, 1}(X, \mathcal{C}) \rightarrow \Psi_{\mathrm{Sc}}^{-\infty, 0}(X, \mathcal{C}) \rightarrow \mathcal{N}_{-\infty} \rightarrow 0,
$$

where the last map is the combined normal operator map.

While the normal operator (together with the principal symbol) suffices to characterize mapping properties on weighted Sobolev spaces, compactness, etc., so far it is not really an operator - it does not act (naturally) on any space of functions. In fact, one can associate a partial convolution action to the normal operators, as was done in [40], but it is more convenient to perform a partial Fourier transform to arrive at a family of operators, which we call indicial operators, on functions on each front face. The cost of the partial Fourier transform is that the range of the combined principal symbol and indicial operator maps, which is $\mathcal{N}_{m}$ for the normal operators, becomes more complicated, except for $\Psi_{\mathrm{Sc}}^{-\infty, 0}(X, \mathcal{C})$, since partial Fourier transform does not have simple mapping properties on conormal distributions. Nonetheless, the resulting simple and natural operator action makes the indicial operator construction worthwhile.

Our next task is thus to construct a multiplicative indicial operator from the normal operators. Rather than proceeding directly, we digress and use oscillatory testing for this purpose as was done in [40]. This approach has the advantage of making the multiplicative properties of the indicial operator transparent. We start by discussing the effect of conjugation of $A$ by oscillatory functions.

Lemma 4.1. - Suppose that $A \in \Psi_{\mathrm{Sc}}^{m, l}(X, \mathcal{C})$ and $\tilde{f} \in \mathcal{C}^{\infty}(X ; \mathbb{R})$. Then

$$
\tilde{A}=\mathrm{e}^{-i \tilde{f} / x} A \mathrm{e}^{i \tilde{f} / x} \in \Psi_{\mathrm{Sc}}^{m, l}(X, \mathcal{C})
$$

Proof. - It is convenient to use the explicit description of $\Psi_{\mathrm{Sc}}(X, \mathcal{C})$ in terms of localization and quantization (3.10). Thus, we may assume that $X=\mathbb{S}_{+}^{n}$. Note that the pull-back of $\tilde{f} / x$ to $\mathbb{R}^{n}$ is a polyhomogeneous symbol of order 1 which we denote by $F$. Then the kernel of $\tilde{A}$ is $\tilde{K}\left(w, w^{\prime}\right)=\mathrm{e}^{i\left(F\left(w^{\prime}\right)-F(w)\right)} K\left(w, w^{\prime}\right)$ where $K$ is the kernel of $A$. But by the fundamental theorem of calculus

$$
F\left(w^{\prime}\right)-F(w)=\sum_{j=1}^{n}\left(w_{j}^{\prime}-w_{j}\right) \int_{0}^{1} \partial_{j} F\left(w+t\left(w^{\prime}-w\right)\right) d t
$$


and $\partial_{j} F$ is a polyhomogeneous symbol of order 0 . Taking into account the rapid decay of $K$ in $W=w-w^{\prime}$ we immediately conclude that $\tilde{K} \in \mathcal{A}^{m, l}\left(\left(\mathbb{S}_{+}^{n}\right)_{\mathrm{Sc}}^{2}, \Delta_{\mathrm{Sc}} ; \mathrm{KD}_{\mathrm{Sc}}^{1 / 2}\right)$ vanishing with all derivatives at $\beta^{*}$ bf $\cup \beta^{*}$ lf $\cup \beta^{*}$ rf, so, returning to the global setting, $\tilde{A} \in \Psi_{\mathrm{Sc}}^{m, l}(X, \mathcal{C})$.

A similar argument in fact shows that the commutator of elements of $\Psi_{\mathrm{Sc}}^{m, l}(X, \mathcal{C})$ with smooth functions $h$ on the original space $X$, i.e. $h \in \mathcal{C}^{\infty}(X) \subset \Psi_{\mathrm{Sc}}^{0,0}(X, \mathcal{C})$, is in fact one order lower than expected, i.e. is in $\Psi_{\mathrm{Sc}}^{m-1, l+1}(X, \mathcal{C})$. We remark that for $h \in \mathcal{C}^{\infty}([X ; \mathcal{C}])$, the corresponding conclusion generally fails.

Lemma 4.2. - Suppose that $A \in \Psi_{\mathrm{Sc}}^{m, l}(X, \mathcal{C})$ and $h \in \mathcal{C}^{\infty}(X)$. Then $B=[A, h] \in$ $\Psi_{\mathrm{Sc}}^{m-1, l+1}(X, \mathcal{C})$.

Proof. - Proceeding as above, we write the kernel of $B$ as $K^{\prime}\left(w, w^{\prime}\right)=\left(h(w)-h\left(w^{\prime}\right)\right) \times$ $K\left(w, w^{\prime}\right)$, where $K$ is the kernel of $A$. Using (4.14) with $h$ in place of $F$, and that $\partial_{j} h$ is a polyhomogeneous symbol of order -1 (it is here that $h \in \mathcal{C}^{\infty}(X)$, rather than $h \in \mathcal{C}^{\infty}([X ; \mathcal{C}])$, is used), we reach our conclusion as in the previous proof.

We next discuss mapping properties on $\mathcal{C}^{\infty}([X ; \mathcal{C}])$.

LemMA 4.3. - If $A \in \Psi_{\mathrm{Sc}}^{m, l}(X, \mathcal{C}), u \in x^{r} \mathcal{C}^{\infty}([X ; \mathcal{C}])$, then $A u \in x^{r+l} \mathcal{C}^{\infty}([X ; \mathcal{C}])$.

Proof. - This result essentially reduces to the fact that $\Psi_{\mathrm{Sc}}(X, \mathcal{C})$ is an algebra. Indeed, write $u=u \cdot 1$, and note that $A u=(A U) 1$ where $B=A U$ denotes the composite of $A$ with the multiplication operator $U$ by $u$. Since the latter is in $x^{r} \operatorname{Diff}_{\mathrm{Sc}}^{0}(X, \mathcal{C})$, hence in $\Psi_{\mathrm{Sc}}^{0, r}(X, \mathcal{C})$, we conclude that $B \in \Psi_{\mathrm{Sc}}^{m, l+r}(X, \mathcal{C})$. Thus, we only have to analyze $B 1$. Again, we can reduce the discussion to a local one. But writing $B$ as the left quantization of a symbol $b(w, \xi)$ as in (3.10), $b$ satisfying (3.14) with $l$ replaced by $l+r$, and writing the oscillatory integral explicitly as a convergent integral, we see that

$$
\begin{aligned}
B 1(w)=(2 \pi)^{-n} \int & \mathrm{e}^{i\left(w-w^{\prime}\right) \cdot \xi}\left\langle w-w^{\prime}\right\rangle^{-2 r}\langle\xi\rangle^{-2 s}\left(1+\Delta_{\xi}\right)^{s} \\
& \times b(w, \xi)\left(1+\Delta_{w^{\prime}}\right)^{r} 1 d w^{\prime} d \xi
\end{aligned}
$$

for $2 r>n, 2 s>n+m$. Changing the variables:

$$
B 1(w)=(2 \pi)^{-n} \int \mathrm{e}^{i W \cdot \xi}\langle W\rangle^{-2 r}\langle\xi\rangle^{-2 s}\left(1+\Delta_{\xi}\right)^{s} b(w, \xi) d W d \xi
$$

This is a convergent integral with $w$ dependence only in $b$. Since

$$
b \in \rho_{\infty}^{-m} \rho_{\partial}^{l+r} \mathcal{C}^{\infty}\left(\left[\mathbb{S}_{+}^{n} ; \mathcal{C}\right] \times \mathbb{S}_{+}^{n}\right),
$$

we conclude that $B 1 \in x^{l+r} \mathcal{C}^{\infty}\left(\left[\mathbb{S}_{+}^{n} ; \mathcal{C}\right]\right)$. Hence, returning to the global setting, $A u \in$ $x^{l+r} \mathcal{C}^{\infty}([X ; \mathcal{C}])$ as claimed.

The previous three lemmas show that if $u=\mathrm{e}^{i \tilde{f} / x} v, v \in \mathcal{C}^{\infty}([X ; \mathcal{C}]), A \in \Psi_{\mathrm{Sc}}^{m, 0}(X, \mathcal{C})$ then $A u=\mathrm{e}^{i \tilde{f} / x} v^{\prime}$ with $v^{\prime} \in \mathcal{C}^{\infty}([X ; \mathcal{C}])$. Moreover, $v^{\prime}$ restricted to the boundary of $[X ; \mathcal{C}]$ only depends on the restriction of $v$ to $\partial[X ; \mathcal{C}]$. It also follows from Lemma 4.2 that if $p \in \partial X$ and $v \in \mathcal{C}^{\infty}([X ; \mathcal{C}])$ vanishes at $\beta_{\mathrm{Sc}}^{-1}(p)$ then $v^{\prime}$ also vanishes there, i.e. composition is local in $X$ (though not in the resolved space $[X ; \mathcal{C}]$ ). Indeed, if $h \in \mathcal{C}^{\infty}(X), v=h v^{\sharp}, u=h u^{\sharp}$, then $A\left(h u^{\sharp}\right)=h A u^{\sharp}+[A, h] u^{\sharp},[A, h] \in \Psi_{\mathrm{Sc}}^{m-1,1}(X, \mathcal{C})$, hence $\mathrm{e}^{-i \tilde{f} / x}[A, h] u^{\sharp} \in x \mathcal{C}^{\infty}([X ; \mathcal{C}])$, so $A(h u)$ vanishes on $\beta_{\mathrm{Sc}}^{-1}(p)$ whenever $h(p)=0$; expanding an arbitrary $v \in \mathcal{C}^{\infty}([X ; \mathcal{C}])$ vanishing 
at $\beta_{\mathrm{Sc}}^{-1}(p)$ in Taylor series to first order then proves the described $X$-locality of the composition. Similarly, if $\tilde{f}(p)=\tilde{f}^{\prime}(p)$ and $d_{y} \tilde{f}(p)=d_{y} \tilde{f}^{\prime}(p)$ (which really just mean that the scattering covectors $d(\tilde{f} / x)$ and $d\left(\tilde{f}^{\prime} / x\right)$ agree at $\left.p\right)$ then $\mathrm{e}^{-i \tilde{f} / x} A \mathrm{e}^{i \tilde{f} / x} v$ and $\mathrm{e}^{-i \tilde{f}^{\prime} / x} A \mathrm{e}^{i \tilde{f}^{\prime} / x} v$ agree at $p$ (this can be proved similarly to the previous lemmas; it will also follow from our explicit calculation below).

This allows us to define the indicial operators of $A$ at the boundary hypersurfaces of $[X ; \mathcal{C}]$. The $a$-indicial operators will be operators on the fibers of the blow-down map resolving $C_{a}$, so in the Euclidean setting, which we discuss in this paragraph, they will e.g. act on Schwartz functions on $X^{a}$, identified as a fiber of the blow-down map. For each point $p \in C_{a}^{\prime}$, we will get such an indicial operator for each $\xi_{a} \in X_{a}^{*}$, i.e. the indicial operators will be maps from an appropriate compactification of ${ }^{\mathrm{sc}} T_{C_{a}^{\prime}}^{*} X_{a}$ to operators on $\mathcal{S}\left(X^{a}\right)$. Their kernels arise essentially by taking the (partial) Fourier transform of the normal operator $N_{C_{a}}$ in $W_{a}$. This behavior of indicial operators corresponds to the fact that near $C_{a}^{\prime}, A \in \Psi_{\mathrm{Sc}}^{m, 0}(X, \mathcal{C})$ can be regarded as a (non-classical!) pseudo-differential operator in the free variables $\left(w_{a}, \xi_{a}\right)$ with values in bounded operators between Sobolev spaces, e.g. on $L^{2}\left(X_{a}\right)$ if $m=0$ (in fact, with values in $\Psi_{\mathrm{Sc}}^{m, 0}\left(\bar{X}^{a}, \mathcal{C}^{a}\right)$ ). More precisely, $A \in \Psi_{\mathrm{scc}}^{0,0}\left(\bar{X}_{a} ; \mathcal{B}\left(L^{2}\left(X^{a}\right), L^{2}\left(X^{a}\right)\right)\right)$ then. This allows one to understand the indicial operator in terms of the operator-valued principal symbol map in the standard scattering calculus.

In the general geometric setting, the indicial operators would depend on certain choices in general (though the dependence is via unitary equivalence), but if we have a scattering metric on $X$ they can be constructed canonically, so we assume this in what follows. We will also need a more thorough understanding of the structure of $[X ; \mathcal{C}]$, so we also discuss this below.

Recall first that a scattering metric $g$ on $X$ is a metric in the interior of $X$ (smooth symmetric positive definite 2-cotensor) which is of the form

$$
g=\frac{d x^{2}}{x^{4}}+\frac{h^{\prime}}{x^{2}}
$$

near $\partial X$, where $x$ is a boundary defining function of $X$ and $h^{\prime}$ is a smooth symmetric 2-cotensor on $X$ whose restriction to the boundary, $h$, is positive definite. Thus, $g$ gives a positive definite pairing on ${ }^{\mathrm{sc}} T X$, so it is (a somewhat special) smooth section of ${ }^{\mathrm{sc}} T^{*} X \otimes{ }^{\mathrm{sc}} T^{*} X$. We remark that the choice of such a $g$ fixes $x$ up to the addition of functions in $x^{2} \mathcal{C}^{\infty}(X)$.

Next, we recall the definition of the relative scattering tangent bundle ${ }^{\mathrm{sc}} T(C ; X)$ of a closed embedded submanifold $C$ of $\partial X$ from [40].

DEFINITION 4.4. - For a closed embedded submanifold $C$ of $\partial X$, the relative scattering tangent bundle ${ }^{\mathrm{sc}} T(C ; X)$ of $C$ in $X$ is the subbundle of ${ }^{\mathrm{sc}} T_{C} X$ consisting of $v \in{ }^{\mathrm{sc}} T_{p} X, p \in C$, for which there exists

$$
V \in \mathcal{V}_{\mathrm{sc}}(X ; C) \subset \mathcal{V}_{\mathrm{sc}}(X)
$$

with $V_{p}=v$. Here

$$
\mathcal{V}_{\mathrm{sc}}(X ; C)=x \mathcal{V}_{\mathrm{b}}(X ; C)=x\left\{V \in \mathcal{V}_{\mathrm{b}}(X): V \text { is tangent to } C\right\}
$$

and tangency is defined using the (non-injective) inclusion map ${ }^{\mathrm{b}} T X \rightarrow T X$.

Thus, in local coordinates $(x, y, z)$ near $p \in C$ such that $C$ is defined by $x=0, y=0$, ${ }^{\text {sc }} T(C ; X)$ is spanned by $x^{2} \partial_{x}$ and $x \partial_{z_{j}}, j=1, \ldots, m-1$, where $n-m$ is the codimension on $C$ in $\partial X$. In the case of Euclidean scattering, $X=\mathbb{S}_{+}^{n}, C=\partial \bar{X}_{a}, g$ the Euclidean metric, ${ }^{\mathrm{sc}} T(C ; X)$ is naturally isomorphic to ${ }^{\mathrm{sc}} T_{C} \bar{X}_{a}$, i.e. it should be regarded as the bundle of scattering tangent vectors of the collision plane at infinity, spanned by $\partial_{\left(w_{a}\right)_{j}}, j=1, \ldots, m$, $m=\operatorname{dim} X_{a}$. 
For $C=C_{a} \in \mathcal{C}$, the metric $g$ defines the orthocomplement $\left({ }^{\mathrm{sc}} T(C ; X)\right)^{\perp}$ of ${ }^{\mathrm{sc}} T(C ; X)$ in ${ }^{\mathrm{sc}} T_{C} X$.

DEFINITION 4.5. - Given $g$, a scattering metric on $X$, the subbundle of ${ }^{\mathrm{sc}} T_{C}^{*} X$ consisting of covectors that annihilate $\left({ }^{\mathrm{sc}} T(C ; X)\right)^{\perp}$, is denoted by ${ }^{\mathrm{sc}} T^{*}(C ; X)$; we say that it is the relative scattering cotangent bundle of $C$ in $X$.

This bundle of course depends on $g$. In the case of Euclidean scattering, ${ }^{\text {sc }} T^{*}(C ; X)$ is naturally isomorphic to ${ }^{\mathrm{sc}} T_{C}^{*} \bar{X}_{a}$ and is spanned by $d\left(w_{a}\right)_{j}, j=1, \ldots, m$.

We now choose local coordinates $(x, y, z)$ near $p \in C$ such that $C$ is defined by $x=0, y=0$, and such that $x \partial_{y_{j}}$ give an orthonormal basis of $\left({ }^{\mathrm{sc}} T(C ; X)\right)^{\perp}$. Note that a basis of ${ }^{\mathrm{sc}} T(C ; X)$ is given by $x^{2} \partial_{x}$ and $x \partial_{z_{j}}$, while a basis of ${ }^{\mathrm{sc}} T^{*}(C ; X)$ is given by $x^{-2} d x, x^{-1} d z_{j}$. A covector in ${ }^{\mathrm{sc}} T^{*} X$ can be written in these local coordinates as

$$
\tau \frac{d x}{x^{2}}+\mu \cdot \frac{d y}{x}+\nu \cdot \frac{d z}{x}
$$

We will write this as

$$
\tau_{a} \frac{d x}{x^{2}}+\mu_{a} \cdot \frac{d y_{a}}{x}+\nu_{a} \cdot \frac{d z_{a}}{x}
$$

to emphasize the element $C=C_{a}$ of $\mathcal{C}$ around which the local coordinates are centered. Thus, local coordinates on ${ }^{\mathrm{sc}} T_{\partial X}^{*} X$ are given by $(y, z, \tau, \mu, \nu)$, while on ${ }^{\mathrm{sc}} T^{*}(C ; X)$ by $(z, \tau, \nu)=$ $\left(z_{a}, \tau_{a}, \nu_{a}\right)$. Note also that at $C$ the metric function of $h$ is of the form $|\mu|^{2}+\tilde{h}(z, \nu)$ with $|\mu|$ denoting the Euclidean length of $\mu$ and $\tilde{h}$ is the metric function of the restriction of $h$ to $T C$; the metric function of $g$ (also denoted by $g$ ) is thus

$$
g=\tau^{2}+\tilde{h}+|\mu|^{2}
$$

there.

Now if $C=C_{a}, C_{b} \in \mathcal{C}$ with $C_{a} \subset C_{b}$, we can further adjust our coordinates so that $C_{b}$ is defined by $x=0, y^{\prime}=0$, for some splitting $y=\left(y^{\prime}, y^{\prime \prime}\right)$. With the corresponding splitting of the dual variable, $\mu=\left(\mu^{\prime}, \mu^{\prime \prime}\right)$, we obtain a well-defined projection

$$
\begin{gathered}
\pi_{b a}:{ }^{\mathrm{sc}} T_{C_{a}}^{*}\left(C_{b} ; X\right) \rightarrow{ }^{\mathrm{sc}} T^{*}\left(C_{a} ; X\right), \\
\pi_{b a}\left(0, z, \tau, \mu^{\prime \prime}, \nu\right)=(z, \tau, \nu) .
\end{gathered}
$$

In the Euclidean setting this is just the obvious projection

$$
\pi_{b a}:{ }^{\mathrm{sc}} T_{\partial \bar{X}_{a}}^{*} \bar{X}_{b} \rightarrow{ }^{\mathrm{sc}} T_{\partial \bar{X}_{a}}^{*} \bar{X}_{a}
$$

under the inclusion $\bar{X}_{a} \subset \bar{X}_{b}$. We write $\pi$ for the collection of these maps.

Before we define the indicial operators, we need to analyze the structure of the lift of $C_{a}$ to $[X ; \mathcal{C}]$. For $C_{a} \in \mathcal{C}$ let

$$
\begin{aligned}
& \mathcal{C}_{a}=\left\{C_{b} \in \mathcal{C}: C_{b} \subsetneq C_{a}\right\}, \\
& \mathcal{C}^{a}=\left\{C_{b} \in \mathcal{C}: C_{a} \subsetneq C_{b}\right\} .
\end{aligned}
$$

We carry out the blow-up $[X ; \mathcal{C}]$ by first blowing up $\mathcal{C}_{a}$. Since all elements of $\mathcal{C}_{a}$ are p-submanifolds of $C_{a}$, the lift $\beta\left[X ; \mathcal{C}_{a}\right]^{*} C_{a}$ of $C_{a}$ to $\left[X ; \mathcal{C}_{a}\right]$ is naturally diffeomorphic to

$$
\tilde{C}_{a}=\left[C_{a} ; \mathcal{C}_{a}\right]
$$


Thus, over $C_{a}^{\prime}$, the regular part of $C_{a}, \tilde{C}_{a}$ can be identified with $C_{a}$. The front face of the new blow-up, i.e. of the blow up of $\beta\left[X ; \mathcal{C}_{a}\right]^{*} C_{a}$ in $\left[X ; \mathcal{C}_{a}\right]$ is thus a hemisphere (i.e. ball) bundle over $\tilde{C}_{a}$, namely $S^{+} N \tilde{C}_{a}$. We write the bundle projection, which is just the restriction of the new blow-down map to the front face, $S^{+} N \tilde{C}_{a}$ as

$$
\rho_{a}: S^{+} N \tilde{C}_{a} \rightarrow \tilde{C}_{a}
$$

In the Euclidean setting, these fibers can be naturally identified with $\bar{X}^{a}$ via the projection $\pi^{a}$ (extended as in Lemma 2.5). Every remaining blow up in $[X ; \mathcal{C}]$ concerns submanifolds that are either disjoint from this new front face or are the lift of elements of $\mathcal{C}^{a}$. The former do not affect the structure near the new front face, $S^{+} N \tilde{C}_{a}=\beta\left[X ; \mathcal{C}_{a} ; C_{a}\right]^{*} C_{a}$, while the latter, which are given by the lifts of elements of $\mathcal{C}^{a}$, correspond to blow ups that can be performed in the fibers of $S^{+} N \tilde{C}_{a}$. Note that the lift of $C_{b} \in \mathcal{C}^{a}$ meets the new front face only at its boundary since all $C_{b}$ are subsets of $\partial X$. In particular, the lift $\beta_{\mathrm{Sc}}^{*} C_{a}$ of $C_{a}$ to $[X ; \mathcal{C}]$ fibers over $\tilde{C}_{a}$ and the fibers are diffeomorphic to a hemisphere (i.e. ball) with certain boundary submanifolds blown up. More specifically, the intersection of $\beta\left[X ; \mathcal{C}_{a} ; C_{a}\right]^{*} C_{b}, C_{b} \in \mathcal{C}^{a}$, with the front face $S^{+} N \tilde{C}_{a}$ is the image of $T \beta\left[X ; \mathcal{C}_{a}\right]^{*} C_{b}$ under the quotients; $\beta_{\mathrm{Sc}}^{*} C_{a}$ is obtained by blowing these up in $S^{+} N \tilde{C}_{a}$. Hence, the fiber of $\beta_{\mathrm{Sc}}^{*} C_{a}$ over $p \in \tilde{C}_{a}$ is given by $\left[S^{+} N_{q} C_{a} ; T_{q} \mathcal{C}^{a}\right]$ where $q=\beta\left[X ; \mathcal{C}_{a}\right](p) \in C_{a}$. In particular, in the Euclidean setting, the fibers of $\beta_{\mathrm{Sc}}^{*} C_{a}$ over $\tilde{C}_{a}$ can be naturally identified with $\left[\bar{X}^{a} ; \mathcal{C}^{a}\right]$ via $\pi^{a}$. Thus, we have the following commutative diagrams:
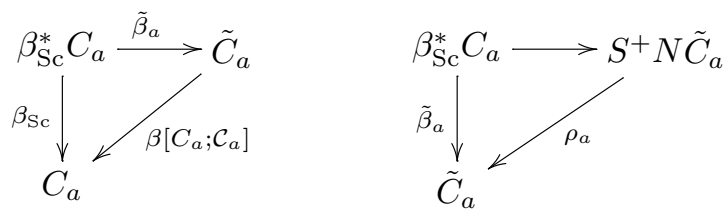

with $\tilde{\beta}_{a}$ being the fibration to the base $\tilde{C}_{a}$.

We now define ${ }^{\mathrm{sc}} T^{*}\left(\tilde{C}_{a} ; X\right)$, denote the pull-back of ${ }^{\mathrm{sc}} T^{*}\left(C_{a} ; X\right)$ by the blow-down map $\beta\left[C_{a} ; \mathcal{C}_{a}\right]$ :

$$
{ }^{\mathrm{sc}} T^{*}\left(\tilde{C}_{a} ; X\right)=\beta\left[C_{a} ; \mathcal{C}_{a}\right]^{* \mathrm{sc}} T^{*}\left(C_{a} ; X\right) .
$$

If $C_{a} \subset C_{b}$ then $\pi_{b a}$ lifts to a map

$$
\tilde{\pi}_{b a}:{ }^{\mathrm{sc}} T_{\beta\left[C_{b} ; \mathcal{C}_{b}\right]^{*} C_{a}}\left(\tilde{C}_{b} ; X\right) \rightarrow{ }^{\mathrm{sc}} T^{*}\left(\tilde{C}_{a} ; X\right) .
$$

We recall from [40, Section 4] that the interior of the fibers $S^{+} N_{p} \tilde{C}_{a}=\rho_{a}^{-1}(p)$ of $\rho_{a}: S^{+} N \tilde{C}_{a} \rightarrow \tilde{C}_{a}, p \in \tilde{C}_{a}$, possess a natural transitive free affine action by the quotient bundle $\left(\beta\left[X ; \mathcal{C}_{a}\right]_{p}^{* \mathrm{sc}} T X\right){ }^{\mathrm{sc}} T_{p}\left(\tilde{C}_{a} ; X\right)$. Thus, the tangent space of $S^{+} N_{p} \tilde{C}_{a}$ at every point $q \in \operatorname{int}\left(S^{+} N_{p} \tilde{C}_{a}\right)$ can be naturally identified with $\left(\beta\left[X ; \mathcal{C}_{a}\right]_{p}^{* \mathrm{sc}} T X\right) /{ }^{\mathrm{sc}} T_{p}\left(\tilde{C}_{a} ; X\right)$, hence with the tangent space at other $q^{\prime} \in \operatorname{int}\left(S^{+} N_{p} \tilde{C}_{a}\right)$.

For each operator $A \in \Psi_{\mathrm{Sc}}^{m, l}(X, \mathcal{C})$, the $C_{a}$-indicial operator of $A$, denoted by $\hat{A}_{a, l}$, will be a collection of operators, one for each $\zeta \in{ }^{\mathrm{sc}} T_{p}^{*}\left(\tilde{C}_{a} ; X\right)$, acting on functions on the fiber $\tilde{\beta}_{a}^{-1}(p)$ of $\tilde{\beta}_{a}$. So suppose that $u \in \dot{\mathcal{C}}^{\infty}\left(\tilde{\beta}_{a}^{-1}(p)\right)$; we need to define $\hat{A}_{a}(\zeta) u$. For this purpose choose $\tilde{f} \in \mathcal{C}^{\infty}(X ; \mathbb{R})$ such that $d(\tilde{f} / x)$, evaluated at $\beta\left[C_{a} ; \mathcal{C}_{a}\right](p)$, is equal to $\zeta$. Then let $\tilde{A}=\mathrm{e}^{-i \tilde{f} / x} x^{-l} A \mathrm{e}^{i \tilde{f} / x} \in \Psi_{\mathrm{Sc}}^{m, 0}(X, \mathcal{C})$, and choose $u^{\prime} \in \mathcal{C}^{\infty}([X ; \mathcal{C}])$ such that $\left.u^{\prime}\right|_{\tilde{\beta}_{a}^{-1}(p)}=u$. Then

$$
\hat{A}_{a, l}(\zeta) u=\left.\left(\tilde{A} u^{\prime}\right)\right|_{\tilde{\beta}_{a}^{-1}(p)},
$$


which is independent of all the choices we made. This can be shown by an argument which is analogous to the proof of the preceding lemmas, but it will also follow from the explicit calculation we make below leading to (4.48). If $l \neq 0$, then $\hat{A}_{a, l}$ would a priori depend on the choice of $x$ up to $\mathcal{O}\left(x^{2}\right)$ terms, but the choice of the scattering metric $g$ fixes $x$ up to such terms. We often simplify (and thereby abuse) the notation and drop the index $l$, i.e. we write $\hat{A}_{a}=\hat{A}_{a, l}$, when the value of $l$ is understood. Before discussing the $C_{a}$-indicial operators of $A \in \Psi_{\mathrm{Sc}}^{m, l}(X, \mathcal{C})$ in detail, we discuss how we can combine them into a single object.

In the case of Euclidean many-body scattering, $C_{a}=\partial \bar{X}_{a}$ and $\hat{A}_{a, l}$ is a function on $\beta_{a}^{* s c} T_{C_{a}}^{*} \bar{X}_{a}$ with values in operators on $\mathcal{S}\left(X^{a}\right)$; here

$$
\beta_{a}=\beta\left[C_{a} ; \mathcal{C}_{a}\right]: \tilde{C}_{a}=\left[C_{a} ; \mathcal{C}_{a}\right] \rightarrow C_{a}
$$

is the blow-down map. Note that $\beta_{a}$ is simply the restriction of $\beta\left[\bar{X}_{a} ; \mathcal{C}_{a}\right]$ to the lift $\tilde{C}_{a}=$ $\beta\left[\bar{X}_{a} ; \mathcal{C}_{a}\right]^{*} C_{a}$. In fact,

$$
\hat{A}_{a, l} \in \mathcal{C}^{\infty}\left(\beta_{a}^{* \mathrm{sc}} T_{\partial \bar{X}_{a}}^{*} \bar{X}_{a}, \Psi_{\mathrm{Sc}}^{m, 0}\left(\bar{X}^{a}, \mathcal{C}^{a}\right)\right)
$$

as we show shortly. Note that if $Z$ is a (not necessarily compact) manifold with corners and $(\tilde{X}, \tilde{\mathcal{C}})$ is a many-body space (in (4.36) we take $Z=\beta_{a}^{* \mathrm{sc}} T_{\partial \bar{X}_{a}}^{*} \bar{X}_{a}$ and $\left(\bar{X}^{a}, \mathcal{C}^{a}\right)$ for the manybody space), it makes perfectly good sense to talk about $\mathcal{C}^{\infty}\left(Z, \Psi_{\mathrm{Sc}}^{m, l}(\tilde{X}, \tilde{\mathcal{C}})\right)$, i.e. about smooth functions on $Z$ with values in $\Psi_{\mathrm{Sc}}^{m, l}(\tilde{X}, \tilde{\mathcal{C}})$. The topology on $\Psi_{\mathrm{Sc}}^{m, l}(\tilde{X}, \tilde{\mathcal{C}})$ is the standard one, namely that of conormal distributions on $\tilde{X}_{\mathrm{Sc}}^{2}$, conormal to $\Delta_{\mathrm{Sc}}$, vanishing to infinite order at $\beta^{*}$ bf $\cup \beta^{*}$ lf $\cup \beta^{*}$ rf, $\beta: \tilde{X}_{\mathrm{Sc}}^{2} \rightarrow \tilde{X}_{\mathrm{b}}^{2}$ the blow-down map. This is equivalent to the topology arising by localizing operators $A \in \Psi_{\mathrm{Sc}}^{m, l}(\tilde{X}, \tilde{\mathcal{C}})$ as in (3.20), and using the topology of the symbol spaces on the local pieces, i.e., with the notation of (3.14) and (3.8), of $\rho_{\infty}^{-m} \rho_{\partial}^{l} \mathcal{C}^{\infty}\left(\left[\mathbb{S}_{+}^{n} ; \tilde{\mathcal{C}}\right] \times \mathbb{S}_{+}^{n}\right)$ and $\mathcal{A}^{-m, l}\left(\left[\mathbb{S}_{+}^{n} ; \tilde{\mathcal{C}}\right] \times \mathbb{S}_{+}^{n}\right)$, in the polyhomogeneous and non-polyhomogeneous setting respectively (and that of $\dot{\mathcal{C}}^{\infty}\left(\tilde{X} \times \tilde{X} ;{ }^{\text {sc }} \Omega_{R}\right)$ for the remainder term).

We need to generalize this example to accommodate the geometric setting. It should be kept in mind throughout following discussion that $Z$ is simply a 'parameter space'. So suppose first that $\phi: E \rightarrow Z$ is a fibration of manifolds with corners with fiber $\tilde{X}$, a manifold with boundary, $\tilde{\mathcal{C}}_{E}$ a cleanly intersecting family of p-submanifolds of $E$ which is fibered over $Z$ with fiber $\tilde{\mathcal{C}}$, a cleanly intersecting family of p-submanifolds of $\partial \tilde{X}$ that gives rise to a many-body space $(\tilde{X}, \tilde{\mathcal{C}})$. That is, we suppose that there is an open cover $\left\{U_{j}: j \in J\right\}$ of $Z$ such that $\left(\phi^{-1}\left(U_{j}\right), \tilde{\mathcal{C}}_{E} \cap \phi^{-1}\left(U_{j}\right)\right)$ is diffeomorphic to $U_{j} \times(\tilde{X}, \tilde{\mathcal{C}})$; we denote the diffeomorphism by $\psi_{j}$. Let $\partial_{\phi} E$ denote the fiber-boundary of $E$, i.e. locally it is given by $U_{j} \times \partial \tilde{X}$ (under the identification $\psi_{j}$ ). The algebra $\Psi_{\mathrm{Sc}, \phi}^{\infty,-\infty}\left(E, \mathcal{C}_{E}\right)$ is then defined as the algebra of operators $A$ acting on, say, functions $u \in \mathcal{C}^{\infty}(E)$ which vanish to infinite order at $\partial_{\phi} E$, with the following local characterization. For each $U_{j}$ there is an operator $A_{j}^{\prime} \in \mathcal{C}^{\infty}\left(U_{j} ; \Psi_{\mathrm{Sc}}^{\infty,-\infty}(\tilde{X}, \tilde{\mathcal{C}})\right)$ such that for $u \in \mathcal{C}^{\infty}(E)$ with $\operatorname{supp} u \subset \phi^{-1}\left(U_{j}\right)$ and vanishing to infinite order at $\partial_{\phi} E, A u=\psi_{j}^{*} A_{j}^{\prime}\left(\psi_{j}^{-1}\right)^{*} u$.

This local description does not depend on any choices. Indeed, the local definition is equivalent to saying that the distribution kernel $K_{A}$ of $A$ on the fiber-product $E \times_{Z} E$ (with values in scattering densities on the fiber $\tilde{X}$ from the right factor, to be precise) is conormal on the appropriate blow-up $E_{\mathrm{Sc}, Z}^{2}$ of $E \times{ }_{Z} E$. Here $K_{A}$ gives rise to the operator $A$ by fiber-integration

$$
A u(w, z)=\int K_{A}\left(w, w^{\prime}, z\right) u\left(w^{\prime}, z\right)\left|d w^{\prime}\right|
$$


where $z$ gives coordinates on $Z, w$ and $w^{\prime}$ are variables in the left and right factor of the fiber $\tilde{X}$ respectively, and we wrote $K_{A}=K_{A}\left(w, w^{\prime}, z\right)\left|d w^{\prime}\right|$. Indeed, following the discussion at the beginning of the previous section, we take $E_{\mathrm{b}, Z}^{2}$ to be the blow-up of $\partial_{\phi} E \times{ }_{Z} \partial_{\phi} E$ in $E \times{ }_{Z} E$, $\Delta_{\mathrm{b}, \phi}$ the lift of the fiber-diagonal, $\partial_{\phi} \Delta_{\mathrm{b}, \phi}$ its fiber-boundary which we identify with $\partial_{\phi} E, \tilde{\mathcal{C}}_{E}^{\prime}$ the image of $\tilde{\mathcal{C}}_{E}$ under this identification, and $E_{\mathrm{Sc}, Z}^{2}$ the blow-up $\left[E_{\mathrm{b}, Z}^{2} ; \tilde{\mathcal{C}}_{E}^{\prime}\right]$. Then the definition of $\Psi_{\mathrm{Sc}, \phi}^{\infty,-\infty}\left(E, \mathcal{C}_{E}\right)$ is given by modifying (3.6) the natural way. Since all blowups can be done in the fibers over $Z$ (i.e. $Z$ can be regarded as a parameter), this description indeed agrees with local definition given above.

This intrinsic definition of $\Psi_{\mathrm{Sc}, \phi}^{\infty,-\infty}\left(E, \mathcal{C}_{E}\right)$ given in the previous paragraph automatically extends even to the setting where the fibration $\phi$ is transversal to the collection $\mathcal{C}_{E}$, each fiber of $\phi$ being diffeomorphic to $\tilde{X}$. Note that in general there are no diffeomorphisms $\psi_{j}$ even locally such that image of $\mathcal{C}_{E}$ takes a product form as above, though such diffeomorphisms exist, for example, if $\mathcal{C}_{E}$ is locally linearizable. In particular, we can take $Z={ }^{\mathrm{sc}} T^{*}\left(\tilde{C}_{a} ; X\right), E$ to be the pull-back of $Z$ to $S^{+} N \tilde{C}_{a}$ by $\rho_{a}, \phi: E \rightarrow Z$ the map $\rho_{a}^{\sharp}$ induced by the pull-back,

$$
E=\rho_{a}^{* \mathrm{sc}} T^{*}\left(\tilde{C}_{a} ; X\right), \quad \rho_{a}^{\sharp}: E \rightarrow{ }^{\mathrm{sc}} T^{*}\left(\tilde{C}_{a} ; X\right) .
$$

Thus, $E$ is a vector bundle over $S^{+} N \tilde{C}_{a}$ with projection $\pi$. Finally, we let $\mathcal{C}_{E}$ consist of the inverse images under $\pi$ of the lifts of $C_{b} \in \mathcal{C}^{a}$ to $\left[X ; \mathcal{C}_{a} ; C_{a}\right]$ intersected with the new front face, $S^{+} N \tilde{C}_{a}$; in fact, we also add $\partial_{\phi} E$ to $\mathcal{C}_{E}$ to play the role of $C_{0}$ in $\mathcal{C}$. We are then in the setting discussed above, so we have defined

$$
\Psi_{\mathrm{Sc}, \rho_{a}^{\sharp}}^{\infty,-\infty}\left(\rho_{a}^{* \mathrm{sc}} T^{*}\left(\tilde{C}_{a} ; X\right), \tilde{\mathcal{C}}_{a}\right), \quad \tilde{\mathcal{C}}_{a}=\pi^{-1}\left(S^{+} N \tilde{C}_{a} \cap \beta\left[X ; \mathcal{C}_{a} ; C_{a}\right]^{*} \mathcal{C}^{a}\right) \cup\left\{\partial_{\phi} E\right\} .
$$

Recall that for $C_{b} \in \mathcal{C}^{a}$,

$$
S^{+} N \tilde{C}_{a} \cap \beta\left[X ; \mathcal{C}_{a} ; C_{a}\right]^{*} C_{b}=T \beta\left[X ; \mathcal{C}_{a}\right]^{*} C_{b},
$$

the right hand side understood as the image of the tangent space under the quotient map. We are now ready to prove the following proposition.

Proposition 4.6. - Suppose that $A \in \Psi_{\mathrm{Sc}}^{r, l}(X, \mathcal{C})$. Then the indicial operators of A satisfy

$$
\hat{A}_{a, l} \in \Psi_{\mathrm{Sc}, \rho_{a}^{\sharp}}^{r, 0}\left(\rho_{a}^{* \mathrm{sc}} T^{*}\left(\tilde{C}_{a} ; X\right), \tilde{\mathcal{C}}_{a}\right) .
$$

Proof. - We prove this statement by finding $\hat{A}_{a}(\zeta)$ explicitly in terms of local coordinates. To simplify the notation we assume that $A \in \Psi_{\mathrm{Sc}}^{r, 0}(X, \mathcal{C})$. We identify $X$ with $\mathbb{S}_{+}^{n}$ locally so that $C_{a}$ is given by $x=0, y=0$. In the interior of $\beta_{\mathrm{Sc}}^{*} C_{a}$ we can use the same coordinates as at the front face of $\left[X ; C_{a}\right]$, i.e. the ones given in (2.7)-(2.8). So suppose that $u^{\prime}$ is supported in the region of validity of these coordinates. Then

$$
A u^{\prime}(w)=\int K\left(w, w^{\prime}\right) u^{\prime}\left(w^{\prime}\right) d w^{\prime}=\int \tilde{a}(w, W) u^{\prime}(w-W) d W
$$

with $\tilde{a}$ as in (3.11). Here the integral is understood as a distributional pairing in general, but it actually converges if $r<-n$. We now consider the coordinates (2.7) on the both factors, i.e. we take $\left(x^{\prime}, Y^{\prime}, z^{\prime}\right)$ corresponding to $w^{\prime}=w-W$, and $(x, Y, z)$ corresponding to $w$. Expressing 
$\left(x^{\prime}, Y^{\prime}, z^{\prime}\right)$ in terms of $(x, Y, z)$ and $W$ (using $\left.w^{\prime}=w-W\right)$ gives

$$
x^{\prime}=x\left(1-x\left(W_{a}\right)_{m}\right)^{-1}, \quad z_{j}^{\prime}=\frac{z_{j}-x\left(W_{a}\right)_{j}}{1-x\left(W_{a}\right)_{m}}, \quad Y_{j}^{\prime}=Y_{j}-\left(W^{a}\right)_{j},
$$

where we wrote $W=\left(W_{a}, W^{a}\right)$ and $\left(W_{a}\right)_{j},\left(W^{a}\right)_{j}$ denote the components of $W_{a}$ and $W^{a}$ respectively. Thus, (4.42) yields

$$
A u^{\prime}(x, Y, z)=\int \tilde{a}(x, Y, z, W) u^{\prime}\left(\frac{x}{1-x\left(W_{a}\right)_{m}}, Y-W^{a}, \frac{z_{j}-x\left(W_{a}\right)_{j}}{1-x\left(W_{a}\right)_{m}}\right) d W
$$

Evaluating at $x=0$ gives

$$
\begin{aligned}
A u^{\prime}(0, Y, z) & =\int \tilde{a}(0, Y, z, W) u^{\prime}\left(0, Y-W^{a}, z\right) d W \\
& =\int\left(\int \tilde{a}(0, Y, z, W) d W_{a}\right) u^{\prime}\left(0, Y-W^{a}, z\right) d W^{a} .
\end{aligned}
$$

Since $\tilde{a}$ is the inverse Fourier transform in the $\xi$ variable of the symbol $a$ whose left quantization is $A$, and since the $W_{a}$ integral above can be understood as the Fourier transform in $W_{a}$ evaluated at the origin, we deduce that

(4.46) $A u^{\prime}(0, Y, z)=(2 \pi)^{-(n-m)} \int \mathrm{e}^{i W^{a} \cdot \xi^{a}} a\left(0, Y, z, 0, \xi^{a}\right) u^{\prime}\left(0, Y-W^{a}, z\right) d \xi^{a} d W^{a}$.

Thus, the indicial operator $\hat{A}_{a}((p, 0))$ where $(p, 0) \in{ }^{\mathrm{sc}} T^{*}\left(\tilde{C}_{a} ; X\right)$ is the zero covector above $p=(0,0, z) \in C_{a}$ is given by

$$
\hat{A}_{a}((p, 0)) u(Y)=(2 \pi)^{-(n-m)} \int \mathrm{e}^{i W^{a} \cdot \xi^{a}} a\left(0, Y, z, 0, \xi^{a}\right) u\left(Y-W^{a}\right) d \xi^{a} d W^{a}
$$

i.e. by the left quantization in $\left(Y, \xi^{a}\right)=\left(W^{a}, \xi^{a}\right)$ of $a\left(0, Y, z, 0, \xi^{a}\right)$. Similar results hold for $\hat{A}_{a}(\zeta)$ in general, namely

$$
\hat{A}_{a}\left(z, \xi_{a}\right) u(Y)=(2 \pi)^{-(n-m)} \int \mathrm{e}^{i W^{a} \cdot \xi^{a}} a\left(0, Y, z, \xi_{a}, \xi^{a}\right) u\left(Y-W^{a}\right) d \xi^{a} d W^{a}
$$

Though the local coordinates are only valid in the interior of $\beta_{\mathrm{Sc}}^{*} C_{a}$, hence not at $\tilde{\beta}_{a}^{*} \partial \tilde{C}_{a}$, the continuity of $\tilde{A} u$ up to $\tilde{\beta}_{a}^{*} \partial \tilde{C}_{a}$ shows that (4.48) also holds with $p \in \tilde{C}_{a}$.

The explicit expression, (4.48) shows, in particular, that $\hat{A}_{a}(\zeta) u$ is indeed independent of the extension $u^{\prime}$ of $u$ that we chose, and also of the choice of $\tilde{f}$ with $d(\tilde{f} / x)$ prescribed at $\beta_{\mathrm{Sc}}(p)$. Moreover, also from (4.48), for each $\zeta \in{ }^{\mathrm{sc}} T_{p}^{*}\left(\tilde{C}_{a} ; X\right), p \in \tilde{C}_{a}$,

$$
\hat{A}_{a}(\zeta) \in \Psi_{\mathrm{Sc}}^{r, 0}\left(\rho_{a}^{-1}(p), T_{p} \mathcal{C}^{a}\right)
$$

here we wrote $T_{p} \mathcal{C}^{a}$ for $T_{p} \beta\left[X ; \mathcal{C}_{a}\right]^{*} \mathcal{C}^{a}$ for simplicity. In fact, (4.48) shows the more precise statement which encodes the smooth dependence of $\hat{A}_{a}(\zeta)$ on $\zeta$, namely that

$$
\hat{A}_{a, l} \in \Psi_{\mathrm{Sc}, \rho_{a}^{\sharp}}^{r, 0}\left(\rho_{a}^{* \mathrm{sc}} T^{*}\left(\tilde{C}_{a} ; X\right), \tilde{\mathcal{C}}_{a}\right) .
$$


In the Euclidean setting the many-body space $\left(\rho_{a}^{-1}(p), T_{p} \mathcal{C}^{a}\right)$ can be identified with $\left(\bar{X}^{a}, \mathcal{C}^{a}\right)$, and we can write

$$
\hat{A}_{a}(\zeta) \in \Psi_{\mathrm{Sc}}^{r, 0}\left(\bar{X}^{a}, \mathcal{C}^{a}\right),
$$

and correspondingly

$$
\hat{A}_{a, l} \in \mathcal{C}^{\infty}\left(\beta_{a}^{* \mathrm{sc}} T_{\partial \bar{X}_{a}}^{*} \bar{X}_{a}, \Psi_{\mathrm{Sc}}^{r, 0}\left(\bar{X}^{a}, \mathcal{C}^{a}\right)\right)
$$

as we have claimed.

If $A \in \Psi_{\mathrm{Sc}}^{r, 0}(X, \mathcal{C})$, then the vanishing of $\hat{A}_{a, 0}(\zeta)$ for every $a$ and every $\zeta \in{ }^{\mathrm{sc}} T^{*}\left(\tilde{C}_{a} ; X\right)$ implies, by our explicit formula, that $a \in \mathcal{C}^{\infty}\left([X ; \mathcal{C}] \times \mathbb{S}_{+}^{n}\right)$ vanishes at $(\partial[X ; \mathcal{C}]) \times \mathbb{S}_{+}^{n}$, so $A \in \Psi_{\mathrm{Sc}}^{r, 1}(X, \mathcal{C})$. Thus, the vanishing of $\sigma_{\mathrm{Sc}, r}(A)$ and all indicial operators together, for $A \in \Psi_{\mathrm{Sc}}^{r, 0}(X, \mathcal{C})$, say, implies that $A \in \Psi_{\mathrm{Sc}}^{r-1,1}(X, \mathcal{C})$.

An advantage of the oscillatory testing definition of the indicial operators is that it makes their multiplicative property clear.

Proposition 4.7. - If $A \in \Psi_{\mathrm{Sc}}^{m, l}(X, \mathcal{C}), B \in \Psi_{\mathrm{Sc}}^{m^{\prime}, l^{\prime}}(X, \mathcal{C})$ then

$$
\widehat{A B}_{a, l+l^{\prime}}(\zeta) u=\hat{A}_{a, l}(\zeta) \hat{B}_{a, l^{\prime}}(\zeta) u \text {. }
$$

The indicial operators are very closely related to the normal operators. In fact, in the proof of Proposition 4.6, $K\left(w, w^{\prime}\right)=\tilde{a}\left(w, w-w^{\prime}\right)$ is the kernel of $A$, and its restriction to the front face is $\tilde{a}(0, Y, z, W)$. Thus, the kernel of $\hat{A}_{a}\left(z, \xi_{a}\right)$ is the partial Fourier transform of $\tilde{a}$ in $W_{a}$ :

$$
\hat{A}_{a}\left(z, \xi_{a}\right)\left(Y, W^{a}\right)=\left(\mathcal{F}_{W_{a}} \tilde{a}\right)\left(0, Y, z, \xi_{a}, W^{a}\right) .
$$

This also shows that the range of the indicial operator map is somewhat complicated. Namely, partial Fourier transform does not respect the conormal singularity of $\tilde{a}$ at $W=0$. However, for operators in $A \in \Psi_{\mathrm{Sc}}^{-\infty, 0}(X, \mathcal{C})$ this problem does not arise: $\tilde{a}$ then is simply in Schwartz in $W$, including smoothness at the origin, hence its partial Fourier transform will have exactly the same properties.

Corresponding to the matching condition, (4.8), for the normal operators at the intersections of the front faces, there is a matching condition for indicial operators. Namely, the indicial operators are related via the projections $\tilde{\pi}_{b a}$. Thus, if $\zeta \in{ }^{\mathrm{sc}} T^{*}\left(\tilde{C}_{a} ; X\right)$, then the indicial operators of $\hat{A}_{a, l}(\zeta)$ are $\hat{A}_{b, l}(\tilde{\zeta})$ where $C_{a} \subset C_{b}, C_{a} \neq C_{b}$, and $\tilde{\zeta} \in{ }^{\mathrm{sc}} T_{\beta\left[C_{b} ; \mathcal{C}_{b}\right]{ }^{*} C_{a}}^{*}\left(\tilde{C}_{b} ; X\right)$ is such that $\tilde{\pi}_{b a}(\tilde{\zeta})=\zeta$. This follows easily from the explicit coordinate form of the indicial operators.

The following proposition is the main reason why a multiplicative indicial operator is important.

Proposition 4.8. - If $A \in \Psi_{\mathrm{Sc}}^{r, 0}(X, \mathcal{C})$ is such that $\sigma_{\mathrm{Sc}, r}(A)$ never vanishes and $\hat{A}_{a}(\zeta)$ is invertible with inverse in $\Psi_{\mathrm{Sc}}^{-r, 0}\left(\rho_{a}^{-1}(p), T_{p} \mathcal{C}^{a}\right)$ (i.e. in $\Psi_{\mathrm{Sc}}^{-r, 0}\left(\bar{X}^{a}, \mathcal{C}^{a}\right)$ in the Euclidean setting) for every $a$ and for every $\zeta \in{ }^{\mathrm{sc}} T^{*}\left(\tilde{C}_{a} ; X\right)$, then there exists a parametrix $P \in \Psi_{\mathrm{Sc}}^{-r, 0}(X, \mathcal{C})$ for $A$ such that $P A-\mathrm{Id}, A P-\mathrm{Id} \in \Psi_{\mathrm{Sc}}^{-\infty, \infty}(X, \mathcal{C})$. Moreover, $P$ has the following properties:

$$
\sigma_{\mathrm{Sc},-r}(P)=\sigma_{\mathrm{Sc}, r}(A)^{-1}, \quad \hat{P}_{a}(\zeta)=\hat{A}_{a}(\zeta)^{-1} .
$$

Proof. - In the construction below we may assume that for each $\zeta$, the $b$-indicial operators of $\hat{A}_{a}(\zeta)^{-1}$ are $\hat{A}_{b}(\tilde{\zeta})^{-1}$ for $\tilde{\zeta}$ with $\tilde{\pi}_{b a}(\tilde{\zeta})=\zeta$, i.e. they 'match up'. Indeed, $\hat{A}_{a}(\zeta) \in$ $\Psi_{\mathrm{Sc}}^{r, 0}\left(\rho_{a}^{-1}(p), T_{p} \mathcal{C}^{a}\right)$, with non-vanishing principal symbol and invertible indicial operators 
$\hat{A}_{b}(\tilde{\zeta}), \tilde{\zeta}$ as above, by the assumption, hence we can apply the proposition inductively, with (4.55) providing the claimed matching for a parametrix $P_{a}(\zeta)$ of $\hat{A}_{a}(\zeta)$, hence, by the usual parametrix argument, for the inverse $\hat{A}_{a}(\zeta)^{-1}$ itself. The matching condition on the indicial operators is vacuous in the two-body type setting, i.e. if $\mathcal{C}=\left\{C_{0}\right\}$, hence we can indeed start the induction from there.

The only non-standard part of the proof is that we can choose $P_{0} \in \Psi_{\mathrm{Sc}}^{-r, 0}(X, \mathcal{C})$ with principal symbol $\sigma_{\mathrm{Sc}, r}(A)^{-1}$ and indicial operators $\hat{A}_{a, 0}(\zeta)^{-1}$, i.e. that these are in the range of the joint principal symbol-indicial operator map. The main issue here is the matching condition involving the principal symbol, expressed by (4.7) for the normal operators, since, as discussed above, partial Fourier transform does not behave too well regarding conormal singularities. Thus, we proceed as follows.

First, note that there is an operator $Q_{0} \in \Psi_{\mathrm{Sc}}^{-r, 0}(X, \mathcal{C})$ with principal symbol $\sigma_{\mathrm{Sc}, r}(A)^{-1}$; this follows from the short exact sequence (4.11), or indeed from (4.4) (note that we are not specifying the normal operators at this stage). Hence, $G=\operatorname{Id}-Q_{0} A \in \Psi_{\mathrm{Sc}}^{0,0}(X, \mathcal{C})$ has vanishing principal symbol, so it is in $\Psi_{\mathrm{Sc}}^{-1,0}(X, \mathcal{C})$. Summing the Neumann series $\sum_{j=1}^{\infty} G^{j}$ asymptotically to some $G_{1} \in \Psi_{\mathrm{Sc}}^{-1,0}(X, \mathcal{C})$ and letting $Q=\left(\operatorname{Id}+G_{1}\right) Q_{0}$ gives $\operatorname{Id}-Q A \in \Psi_{\mathrm{Sc}}^{-\infty, 0}(X, \mathcal{C})$, then a similar right parametrix construction and the standard argument comparing the two parametrices shows that $Q$ satisfies

$$
\operatorname{Id}-Q A, \operatorname{Id}-A Q \in \Psi_{\mathrm{Sc}}^{-\infty, 0}(X, \mathcal{C}) .
$$

In particular, taking indicial operators,

$$
T_{a}(\zeta)=\operatorname{Id}-\hat{Q}_{a}(\zeta) \hat{A}_{a}(\zeta), \quad \operatorname{Id}-\hat{A}(\zeta) \hat{Q}(\zeta) \in \Psi_{\mathrm{Sc}}^{-\infty, 0}\left(\rho_{a}^{-1}(p), T_{p} \mathcal{C}^{a}\right),
$$

and the kernels, which are smooth functions, decay rapidly as $\zeta \rightarrow \infty$. This implies, in particular, that $\left\|T_{a}(\zeta)\right\|_{\mathcal{B}\left(L_{\mathrm{sc}}^{2}\left(\rho_{a}^{-1}(p)\right)\right)} \rightarrow 0$ as $\zeta \rightarrow \infty$, hence $\mathrm{Id}-T_{a}(\zeta)$ is invertible for sufficiently large $\zeta$ without any assumption on the a priori invertibility of $\hat{A}_{a}(\zeta)$, hence $\hat{A}_{a}(\zeta)^{-1}$ exists for large $\zeta$ and differs from $\hat{Q}_{a}(\zeta)$ by a term rapidly decreasing in $\zeta$. In general, for arbitrary $\zeta$, the standard parametrix argument, consisting of multiplying the previous expressions by $\hat{A}_{a}(\zeta)^{-1}$, then shows that

$$
\left(\hat{A}_{a}(\zeta)\right)^{-1}-\hat{Q}_{a}(\zeta) \in \Psi_{\mathrm{Sc}}^{-\infty, 0}\left(\rho_{a}^{-1}(p), T_{p} \mathcal{C}^{a}\right) .
$$

Since the inverse partial Fourier transform maps Schwartz functions to Schwartz functions, we deduce that the normal operators corresponding to $\left(\hat{A}_{a}(\zeta)\right)^{-1}-\hat{Q}_{a}(\zeta)$ are in the space $\mathcal{C}^{\infty}\left(\mathrm{ff}_{C_{a}} ;{ }^{\mathrm{sc}} \Omega_{R}\right)$ with infinite order vanishing on bf, and they satisfy (4.8) as mentioned in the first paragraph. Thus, from the short exact sequence (4.11), or indeed from (4.12), there exists an operator $R \in \Psi_{\mathrm{Sc}}^{-\infty, 0}(X, \mathcal{C})$ such that $\hat{R}_{a}(\zeta)=\left(\hat{A}_{a}(\zeta)\right)^{-1}-\hat{Q}_{a}(\zeta)$.

Let $P_{0}=Q+R$; then $\sigma_{\mathrm{Sc}, r}\left(P_{0}\right)=\sigma_{\mathrm{Sc}, r}(A)^{-1}$, and $\widehat{P}_{0 a}(\zeta)=\left(\hat{A}_{a}(\zeta)\right)^{-1}$ as desired. Hence, proceeding as usual, $E=\operatorname{Id}-P_{0} A \in \Psi_{\mathrm{Sc}}^{0,0}(X, \mathcal{C})$ has vanishing principal symbol and indicial operators, so it is in $\Psi_{\mathrm{Sc}}^{-1,1}(X, \mathcal{C})$. Summing the Neumann series $\sum_{j=1}^{\infty} E^{j}$ asymptotically to some $F \in \Psi_{\mathrm{Sc}}^{-1,1}(X, \mathcal{C})$ and letting $P=(\mathrm{Id}+F) P_{0}$ gives the required left parametrix. A right parametrix can be constructed similarly, and then the usual argument shows that they can be taken to be the same.

For $A \in \Psi_{\mathrm{Sc}}^{m, 0}(X, \mathcal{C})$ self-adjoint, $m>0$, with $\sigma_{\mathrm{Sc}, m}(A)$ never vanishing, we automatically have that $(A-\lambda)^{-1} \in \Psi_{\mathrm{Sc}}^{-m, 0}(X, \mathcal{C})$ for $\lambda \in \mathbb{C} \backslash \mathbb{R}$. Indeed, the indicial operator of $A-\lambda$ at the free face, i.e. the lift of $C_{0}=\partial X$ to $[X ; \mathcal{C}]$, is $\hat{A}_{0}(z, \xi)-\lambda$, i.e. a $\mathbb{C}$-valued function, which is non-zero since the self-adjointness of $A$ implies that $\hat{A}_{0}$ is real, while on the other hand $\operatorname{Im} \lambda \neq 0$. 
Then an inductive argument on the clusters $C_{a}$, starting with $C_{0}$, and using the above proposition, shows first the existence of a parametrix for $\hat{A}_{a}(\zeta)-\lambda$ inside $\Psi_{\mathrm{Sc}}^{-\infty, 0}\left(\rho_{a}^{-1}(p), T_{p} \mathcal{C}^{a}\right)$, which then allows us to conclude the invertibility of $\hat{A}_{a}(\zeta)-\lambda$ in the same space (since we already know that the inverse exists as a bounded operator on $L^{2}\left(\rho_{a}^{-1}(p)\right)$ ), completing the inductive step. (This is essentially the inductive procedure outlined in the first paragraph of the proof.)

Moreover, the blow-up of $(A-\lambda)^{-1}$ in $\Psi_{\mathrm{Sc}}^{-m, 0}(X, \mathcal{C})$ can be analyzed uniformly as $\lambda$ approaches the real axis, see e.g. [9,40]. Therefore, the functional calculus for self-adjoint operators $A$ and the Cauchy integral representation of $\phi(A)$ via almost analytic extensions, as in the work of Helffer and Sjöstrand [10], Dereziński and Gérard [3], see also [9], gives immediately

Proposition 4.9. - Suppose that $A \in \Psi_{\mathrm{Sc}}^{m, 0}(X, \mathcal{C})$ self-adjoint, $m>0$, and $\sigma_{\mathrm{Sc}, m}(A)$ never vanishes. Suppose also that $\phi \in \mathcal{C}_{c}^{\infty}(\mathbb{R})$. Then $\phi(A) \in \Psi_{\mathrm{Sc}}^{-\infty, 0}(X, \mathcal{C})$ and its indicial operators are $\phi\left(\hat{A}_{a}(\zeta)\right)$. If instead we assume $\phi \in S_{\mathrm{phg}}^{-r}(\mathbb{R})$ then $\phi(A) \in \Psi_{\mathrm{Sc}}^{-r m, 0}(X, \mathcal{C})$.

If $m=0$, that is $A \in \Psi_{\mathrm{Sc}}^{0,0}(X, \mathcal{C})$, then $\phi(A) \in \Psi_{\mathrm{Sc}}^{0,0}(X, \mathcal{C})$ without any assumption on the invertibility of $\sigma_{\mathrm{Sc}, 0}(A)$. We thus have:

Proposition 4.10. - Suppose that $A \in \Psi_{\mathrm{Sc}}^{0,0}(X, \mathcal{C})$ is self-adjoint. If $\phi \in \mathcal{C}^{\infty}(\mathbb{R})$ then $\phi(A) \in \Psi_{\mathrm{Sc}}^{0,0}(X, \mathcal{C})$.

Proof. - Since $A$ is bounded, we can replace $\phi$ by a function $\psi \in \mathcal{C}_{c}^{\infty}(\mathbb{R})$ such that $\phi \equiv \psi$ on the spectrum of $A$. Now $\sigma_{\mathrm{Sc}, 0}(A-\lambda)=\sigma_{\mathrm{Sc}, 0}(A)-\lambda$ is invertible for $\lambda \in \mathbb{C} \backslash \mathbb{R}$, so $(A-\lambda)^{-1} \in \Psi_{\mathrm{Sc}}^{0,0}(X, \mathcal{C})$ for $\lambda \notin \mathbb{R}$. Again, $(A-\lambda)^{-1}$ can be analyzed uniformly up to the real axis, and then the Cauchy integral representation of $\psi(A)$ now proves the proposition.

Remark 4.11. - Following through the Cauchy formula also shows that the principal symbol of $\phi(A)$ is $\phi\left(\sigma_{\mathrm{Sc}, 0}(A)\right)$, and the indicial operators are $\phi\left(\hat{A}_{a}(\zeta)\right)$.

\section{The wave front set}

The Sc-wave front set $\mathrm{WF}_{\mathrm{Sc}}(u)$ of a distribution $u$, and the Sc-operator wave front set $\mathrm{WF}_{\mathrm{Sc}}^{\prime}(A)$ of $A \in \Psi_{\mathrm{Sc}}^{m, l}(X, \mathcal{C})$, at infinity will be defined as subsets of the compressed scattering cotangent bundle

$$
{ }^{\mathrm{sc}} \dot{T}^{*} X=\bigcup_{a}^{\mathrm{sc}} T_{C_{a}^{\prime}}^{*}\left(C_{a} ; X\right)
$$

we have defined ${ }^{\mathrm{sc}} T^{*}\left(C_{a} ; X\right)$ in Definition 4.5. This is very similar to the image of the cotangent bundle in the compressed cotangent bundle (the b-cotangent bundle) that Melrose and Sjöstrand used to describe the propagation of singularities for the wave equation in domains with smooth boundaries [23] and also to the corresponding phase space for domains with corners $\Omega, \dot{T}_{\mathrm{b}}^{*} \Omega$, which was the setting for Lebeau's analysis of the singularities of solutions to the wave equation on $\Omega$. Thus, one may think of $\mathrm{WF}_{\mathrm{Sc}}(u)$ as containing less detailed information than $\mathrm{WF}_{\mathrm{sc}}(u)$, in the sense that the former is a subset of the compressed bundle, while the latter is a subset of the non-compressed bundle, ${ }^{\mathrm{sc}} T_{\partial X}^{*} X$. However, there is no simple relationship between these two wave front sets. In particular, neither of these wave front sets can be used to describe the other. Thus, the picture that $\mathrm{WF}_{\mathrm{Sc}}(u)$ contains less detailed information is at least partly incorrect; the two wave front sets are simply different. The fact that $\mathrm{WF}_{\mathrm{Sc}}(u)$ lives on a compressed version of ${ }^{\mathrm{sc}} T_{\partial X}^{*} X$ corresponds to the singular behavior of elements of $\Psi_{\mathrm{Sc}}^{m, l}(X, \mathcal{C})$, as compared to those of $\Psi_{\mathrm{sc}}^{m, l}(X)$. 
As mentioned in the introduction, we make the assumption that $\mathcal{C}$ is locally linearizable. This assumption simplifies the geometry, and it enables us to give a rather explicit description of the wave front sets.

Before proceeding with a discussion of wave front sets, we discuss the basic topology of ${ }^{\mathrm{sc}} \dot{T}^{*} X$. We topologize ${ }^{\mathrm{sc}} \dot{T}^{*} X$ using the projection $\pi:{ }^{\mathrm{sc}} T_{\partial X}^{*} X \rightarrow{ }^{\mathrm{sc}} \dot{T}^{*} X$. We say that a function $f$ on ${ }^{\mathrm{sc}} T_{\partial X}^{*} X$ is $\pi$-invariant if $f(\zeta)=f\left(\zeta^{\prime}\right)$ whenever $\pi(\zeta)=\pi\left(\zeta^{\prime}\right)$; in this case $f$ induces a function $f_{\pi}$ on ${ }^{\mathrm{sc}} \dot{T}^{*} X$ with $f_{\pi} \circ \pi=f$. By definition, $C \subset{ }^{\mathrm{sc}} \dot{T}^{*} X$ is closed if and only if $\pi^{-1}(C)$ is closed, so if $f$ is continuous on ${ }^{\mathrm{sc}} T^{*} X$ and $\pi$-invariant, then $f_{\pi}$ is continuous on ${ }^{\mathrm{sc}} \dot{T}^{*} X$.

Under our assumption that $\mathcal{C}$ is locally linearizable, if $p \in C_{a}^{\prime}$, we can choose local coordinates $\left(y_{a}, z_{a}\right)$ on $\partial X$ in terms of which all the $C_{b}$ satisfying $p \in C_{b}$ are linear, i.e. they are given by $A_{b} y_{a}=0$ where $A_{b}$ is a (constant) matrix, and $C_{a}$ is given by $y_{a}=0$. Let $\left(\tau_{a}, \mu_{a}, \nu_{a}\right)$ denote the sc-dual variables of $\left(x, y_{a}, z_{a}\right)$ as in (4.22). Choosing such coordinates, $y_{a}, z_{a}, \tau_{a}, \nu_{a}$ are $\pi$-invariant near ${ }^{\mathrm{sc}} T_{p}^{*} X$.

In particular, there are always continuous functions separating points in ${ }^{\mathrm{sc}} \dot{T}^{*} X$ : if $p(\bar{\zeta}) \neq p\left(\bar{\zeta}^{\prime}\right)$ (here $p:{ }^{\text {sc }} \dot{T}^{*} X \rightarrow \partial X$ stands for projection to the base), one can use the pull-back of an appropriate function on $\partial X$, and if $p(\bar{\zeta})=p\left(\bar{\zeta}^{\prime}\right) \in C_{a}^{\prime}$, then $\bar{\zeta}, \bar{\zeta}^{\prime} \in{ }^{\mathrm{sc}} T_{C_{a}^{\prime}}^{*}\left(C_{a} ; X\right)$ are of the form $\left(\bar{z}_{a}, \bar{\tau}_{a}, \bar{\nu}_{a}\right)$ and $\left(\bar{z}_{a}^{\prime}, \bar{\tau}_{a}^{\prime}, \bar{\nu}_{a}^{\prime}\right), \bar{\tau}_{a} \neq \bar{\tau}_{a}^{\prime}$ or $\bar{\nu}_{a} \neq \bar{\nu}_{a}^{\prime}$, and the functions $\zeta \mapsto \tau_{a}, \zeta \mapsto \nu_{a}$, are well-defined and $\pi$-invariant on a neighborhood of $p(\bar{\zeta})$ (this uses that $\mathcal{C}$ is locally linearizable), so multiplying them by the pull-back of a cutoff on $\partial X$ gives globally well-defined separating continuous functions. Thus, ${ }^{\mathrm{sc}} \dot{T}^{*} X$ is Hausdorff.

Note that if $K_{0}$ is a compact subset of ${ }^{\mathrm{sc}} T_{\partial X}^{*} X$, then $K=\pi\left(K_{0}\right)$ is compact, and in fact it is a compact metrizable space, just as in Lebeau's setting [18, Section III]. (The characteristic variety of $\Delta-\lambda$ is an example of such a set $K_{0}$ that is of interest here.) This can also be seen explicitly by showing that $K$ is normal, which we proceed to show.

Fix $\bar{\zeta} \in{ }^{\mathrm{sc}} T_{C_{a}^{\prime}}^{*} \bar{X}_{a}$, write $\bar{\zeta}=\left(\bar{z}_{a}, \bar{\tau}_{a}, \bar{\nu}_{a}\right)$, and choose a neighborhood $U_{0}$ of $\bar{z}_{a}=p(\bar{\zeta})$ in $\partial X$ such that $\overline{U_{0}} \cap C_{b}=\emptyset$ unless $C_{a} \subset C_{b}$. Let $\omega_{\bar{\zeta}}=\omega:{ }^{\text {sc }} \dot{T}_{\overline{U_{0}}}^{*} X \rightarrow \mathbb{R}$ be given by the following $\pi$-invariant function on ${ }^{\mathrm{sc}} T_{\overline{U_{0}}}^{*} X$ (also denoted by $\omega$ ):

$$
\omega(\zeta)=\left|y_{a}\right|^{2}+\left|z_{a}-\bar{z}_{a}\right|^{2}+\left|\tau_{a}-\bar{\tau}_{a}\right|^{2}+\left|\nu_{a}-\bar{\nu}_{a}\right|^{2} \geqslant 0
$$

in the coordinates $\left(y_{a}, z_{a}, \tau_{a}, \mu_{a}, \nu_{a}\right)$. Suppose that $U$ is open in $K, \bar{\zeta} \in U$. Thus, $K^{\prime}=$ $\left(K \cap{ }^{\mathrm{sc}} \dot{T}_{\overline{U_{0}}}^{*} X\right) \backslash U$ is compact, so, unless $K^{\prime}$ is empty, $\omega$ assumes a minimum on it which thus has to be non-negative. But $\omega(\zeta)=0$ implies $y_{a}=0$, so $p(\zeta) \in C_{a}$, and then $z_{a}=\bar{z}_{a}, \tau_{a}=\bar{\tau}_{a}$, $\nu_{a}=\bar{\nu}_{a}$, show that $\zeta=\bar{\zeta}$. Since $\bar{\zeta} \in U$, this shows that there exists $\delta>0$ such that $\omega \geqslant \delta$ on $K$. Replacing $\delta>0$ by possibly a smaller number, we can also assume that $\omega(\zeta)<\delta$ implies $p(\zeta) \in U_{0}$. We thus conclude that if $U$ is a neighborhood of $\bar{\zeta}$ in $K$, then there exists $\delta>0$ such that

$$
\{\zeta \in K: \omega(\zeta)<\delta\} \subset U .
$$

These sets are open since $\omega$ is continuous, hence they form a basis for the topology of $K$ as $\bar{\zeta}$ and $\delta$ vary; it is easy to see that if one restricts both of these to suitable countable sets, one still has a basis. Note that, separating the complement of $U$ from $\bar{\zeta}$ by a level set of $\omega$ shows explicitly that $K$ is regular, and a simple compactness argument using these $\omega_{\bar{\zeta}}$ (composed with cut-off functions on the reals as in the next paragraph) shows that $K$ is normal, hence a compact metrizable space, as claimed.

Composing $\omega$ with a $\mathcal{C}^{\infty}$ function on $\mathbb{R}$ supported near 0 also shows that given any $\bar{\zeta} \in K$ and any neighborhood $U$ of $\bar{\zeta}$ in $K$, one can construct a $\pi$-invariant $\mathcal{C}^{\infty}$ function $f$ on ${ }^{\mathrm{sc}} T_{\partial X}^{*} X$ for 
which $f_{\pi}(\bar{\zeta}) \neq 0$ and $\operatorname{supp} f_{\pi} \cap K \subset U$. This also shows the existence of smooth partitions of unity on $K$, smoothness understood as smoothness for the pull-back to ${ }^{\mathrm{sc}} T^{*} X$.

The definition of $\mathrm{WF}_{\mathrm{Sc}}(u)$ and $\mathrm{WF}_{\mathrm{Sc}}^{\prime}(A)$ will be local in $X$. Thus, we can always work on $\mathbb{S}_{+}^{n}$ instead. Just like when we defined $\Psi_{\mathrm{Sc}}^{m, l}(X, \mathcal{C})$, we will be able to proceed either by giving an explicit description in $\mathbb{S}_{+}^{n}$ via the Fourier transform, or by giving invariant definitions. Throughout this section we follow the construction of the fibred cusp wave front set by Mazzeo and Melrose [19, Section 7]; their proofs can be easily modified to accommodate our setting.

We start with the operator wave front sets, which we only define for 'smoothing operators' $A \in \Psi_{\mathrm{Sc}}^{-\infty, l}(X, \mathcal{C})$ to eliminate the necessity of defining the usual operator wave front set on ${ }^{\mathrm{Sc}} S^{*}[X ; \mathcal{C}]$. The invariant definition proceeds by oscillatory testing.

Definition 5.1. - Suppose that $A \in \Psi_{\mathrm{Sc}}^{-\infty, l}(X, \mathcal{C})$ and $\zeta \in{ }^{\mathrm{sc}} T_{p}^{*}\left(C_{a} ; X\right), p \in C_{a}^{\prime}$. We say that $\zeta \notin \mathrm{WF}_{\mathrm{Sc}}^{\prime}(A)$ if and only if there exist a neighborhood $U$ of $\zeta$ in ${ }^{\mathrm{sc}} \dot{T}^{*} X$ and a neighborhood $V$ of $p$ in $X$ such that $A u \in \mathcal{C}^{\infty}(X)$ for every oscillatory function $u=\mathrm{e}^{i f / x} v, v \in \mathcal{C}^{\infty}([X ; \mathcal{C}])$ with $\pi(\operatorname{graph}(d(f / x))) \cap{ }^{\mathrm{sc}} \dot{T}_{V \cap \partial X}^{*} X \subset U$ and $\operatorname{supp} v \subset \beta_{\mathrm{Sc}}^{-1}(V)$.

This definition implies immediately that $\operatorname{WF}_{\mathrm{Sc}}^{\prime}(A)$ is closed in ${ }^{\mathrm{sc}} \dot{T}^{*} X$,

$$
\begin{gathered}
\mathrm{WF}_{\mathrm{Sc}}^{\prime}(A+B) \subset \mathrm{WF}_{\mathrm{Sc}}^{\prime}(A) \cup \mathrm{WF}_{\mathrm{Sc}}^{\prime}(B), \quad A, B \in \Psi_{\mathrm{Sc}}^{-\infty, l}(X, \mathcal{C}), \\
\mathrm{WF}_{\mathrm{Sc}}^{\prime}(A B) \subset \mathrm{WF}_{\mathrm{Sc}}^{\prime}(A) \cap \mathrm{WF}_{\mathrm{Sc}}^{\prime}(B), \quad A, B \in \Psi_{\mathrm{Sc}}^{-\infty, l}(X, \mathcal{C}) .
\end{gathered}
$$

We can also formulate the definition explicitly. We thus locally identify $X$ with $\mathbb{S}_{+}^{n}$ and consider $A \in \Psi_{\mathrm{Sc}}^{-\infty, l}\left(\mathbb{S}_{+}^{n}, \mathcal{C}\right)$. We also identify ${ }^{\mathrm{sc}} T^{*} \mathbb{S}_{+}^{n}$ with $\mathbb{S}_{+}^{n} \times \mathbb{R}^{n}$. So suppose that $A$ is the left quantization of a symbol $a \in \rho_{\partial}^{l} \mathcal{C}^{\infty}\left(\left[\mathbb{S}_{+}^{n} ; \mathcal{C}\right] \times \mathbb{S}_{+}^{n}\right)$ that vanishes to infinite order at $\left[\mathbb{S}_{+}^{n} ; \mathcal{C}\right] \times \partial \mathbb{S}_{+}^{n}$. Then $\zeta \notin \mathrm{WF}_{\mathrm{Sc}}^{\prime}(A), \zeta \in{ }^{\mathrm{sc}} T_{p}^{*}\left(C_{a} ; X\right), p \in C_{a}^{\prime}$, if and only if there exists a neighborhood $U$ of $\zeta$ in ${ }^{\text {sc }} \dot{T}^{*} \mathbb{S}_{+}^{n}$ such that $a$ vanishes at $U^{\prime} \subset\left(\partial\left[\mathbb{S}_{+}^{n} ; \mathcal{C}\right]\right) \times \mathbb{R}^{n}$ to infinite order where $U^{\prime}$ is the inverse image of $U$ under the composite map

$$
\left(\partial\left[\mathbb{S}_{+}^{n} ; \mathcal{C}\right]\right) \times \mathbb{R}^{n} \stackrel{\beta_{\mathrm{Sc}} \times \mathrm{id}}{\longrightarrow}\left(\partial \mathbb{S}_{+}^{n}\right) \times \mathbb{R}^{n}={ }^{\mathrm{sc}} T_{\mathbb{S}^{n-1}}^{*} \mathbb{S}_{+}^{n} \stackrel{\pi}{\longrightarrow}{ }^{\mathrm{sc}} \dot{T}^{*} \mathbb{S}_{+}^{n}
$$

It follows immediately from the usual formulae relating quantizations and the effect of diffeomorphisms that this definition is independent of such choices. For example, we could have equally well written $A$ as the right quantization of a symbol with similar properties.

The general definition for $A \in \Psi_{\mathrm{Sc}}^{m, l}(X, \mathcal{C})$, again following the paper [19], in the explicit quantization form as in the previous paragraph, would also require the rapid decay of $a$ in an open cone (conic in the cotangent variable, $\xi$, i.e. in the second factor, $\mathbb{R}^{n}$, in (5.6)) that includes $U^{\prime}$. For $A \in \Psi_{\mathrm{Sc}}^{-\infty, l}(X, \mathcal{C}), a$ is rapidly decreasing in every direction as $|\xi| \rightarrow \infty$, so this statement is vacuous, and we recover Definition 5.1. The main point is that if $A \in \Psi_{\mathrm{Sc}}^{0,0}(X, \mathcal{C}), \hat{A}_{a}(\zeta)$ is invertible, then there exists a microlocal parametrix for $A$, i.e. there exists $G \in \Psi_{\mathrm{Sc}}^{0,0}(X, \mathcal{C})$ such that $\mathrm{Id}=A G+R_{R}$, Id $=G A+R_{L}$, with $R_{R}, R_{L} \in \Psi_{\mathrm{Sc}}^{0,0}(X, \mathcal{C}), \zeta \notin \operatorname{WF}_{\mathrm{Sc}}^{\prime}\left(R_{R}\right)$, $\zeta \notin \mathrm{WF}_{\mathrm{Sc}}^{\prime}\left(R_{L}\right)$; see [19, Lemmas 14-15].

More explicitly, we have the following sufficient condition for $\zeta \notin \operatorname{WF}_{\mathrm{Sc}}^{\prime}(A)$, $A \in \Psi_{\mathrm{Sc}}^{-\infty, 0}(X, \mathcal{C})$. Namely, if there is a neighborhood $V$ of $\zeta=\left(0, z_{a}^{0}, \xi_{a}^{0}\right) \in{ }^{\mathrm{sc}} T_{p}^{*}\left(C_{a} ; \mathbb{S}_{+}^{n}\right)$, $p \in C_{a}^{\prime}$, in $\partial \mathbb{S}_{+}^{n} \times \mathbb{R}^{m}$ such that $a$ vanishes to infinite order at every point $\left(q^{\prime}, \xi\right) \in\left(\partial\left[\mathbb{S}_{+}^{n} ; \mathcal{C}\right]\right) \times$ $\mathbb{R}^{n}$ with $\left(\beta_{\mathrm{Sc}}\left(q^{\prime}\right), \xi_{a}\right) \in V$, then $\zeta \notin \operatorname{WF}_{\mathrm{Sc}}^{\prime}(A)$. Note that as $p \in C_{a}^{\prime}$, we can always assume, by reducing the size of $V$ if necessary, that $\left(q, \xi^{a}\right) \in V$ implies $q \in C_{b}^{\prime}$ for some $b$ with $C_{a} \subset C_{b}$. We can see that this condition is sufficient for $\zeta \notin \mathrm{WF}_{\mathrm{Sc}}^{\prime}(A)$ since for nearby $q \in \mathbb{S}^{n-1}$, assuming as 
we may that $q \in C_{b}^{\prime}, C_{a} \subset C_{b}$, the restriction of $\pi$ to ${ }^{\mathrm{sc}} T_{q}^{*} \mathbb{S}_{+}^{n}$ takes the form $\left(q, \xi_{b}, \xi^{b}\right) \mapsto\left(q, \xi_{b}\right)$ and $\xi_{b}$ splits as $\left(\xi_{b}^{\prime}, \xi_{b}^{\prime \prime}\right)$ with $\xi_{b}^{\prime}=\xi_{a}$. Thus, the condition of the previous paragraph holds if we take

$$
U=\bigcup_{b}\left\{\left(q, \xi_{b}\right): q \in C_{b}^{\prime}, \exists \xi_{a}, \xi_{b}^{\prime \prime} \text { s.t. }\left(q, \xi_{a}\right) \in V \text { and } \xi_{b}=\left(\xi_{b}^{\prime}, \xi_{b}^{\prime \prime}\right)\right\} .
$$

The definition of the wave front set of a distribution $u \in \mathcal{C}^{-\infty}(X)$ at $\partial X$ is more complicated. To determine whether $\zeta \in{ }^{\mathrm{sc}} T_{p}^{*}\left(C_{a} ; X\right), p \in C_{a}^{\prime}$, is in $\mathrm{WF}_{\mathrm{Sc}}(u)$, we would like to cut off $u$ to be supported near $p$, i.e. consider $\psi u, \psi \in \mathcal{C}^{\infty}(X), \psi \equiv 1$ near $p$, identify a neighborhood of $p$ with an open set in $\mathbb{S}_{+}^{n}$ near $\partial \mathbb{S}_{+}^{n}$, and consider smoothness of the Fourier transform of $u, \mathcal{F} \psi u$. Indeed, in the two-body setting, hence in the many-body setting if we consider $\zeta \in{ }^{\mathrm{sc}} T_{C_{0}^{\prime}}^{*}\left(C_{0} ; X\right)={ }^{\mathrm{sc}} T_{C_{0}^{\prime}}^{*} X$, written as a covector $\xi \cdot d w$ over $p \in C_{0}^{\prime}$, we have

$$
\zeta \notin \mathrm{WF}_{\mathrm{sc}}(u) \quad \text { iff } \quad \exists \psi \text { as above, s.t. } \mathcal{F} \psi u \text { is smooth near } \xi \text {. }
$$

In the general many-body setting, $\zeta \in{ }^{\mathrm{sc}} T_{p}^{*}\left(C_{a} ; X\right), p \in C_{a}^{\prime}, \zeta$ takes the form $\xi_{a} \cdot d w_{a}$, and correspondingly we would like to say that $\mathcal{F} \psi u$ is Schwartz in a region including the subspace $S$ consisting of all points of the form $\left(\xi_{a}, \xi^{a}\right)$ where $\xi^{a}$ is arbitrary. Here Schwartz takes the place of smooth functions since the region is not compact in $\mathbb{R}^{n}$. However, as shown by the example of ordinary wave front set, we cannot expect that this wave front set behaves reasonably unless the region $U$ is conic near infinity, i.e. unless it is a neighborhood of the closure of $S$ in the radial compactification $\mathbb{S}_{+}^{n}$ of $\mathbb{R}^{n}$. This however introduces the complication that all parallel translates of $S$ intersect $U$, and we are exactly interested in separating from each other the singularities on the various translates of $S$. This problem is not too serious, especially for generalized eigenfunctions of many-body Hamiltonians $H$, but it introduces additional terms into the following definition which is modelled on that of the fibred cusp wave front set by Mazzeo and Melrose [19].

Definition 5.2. - We say that

$$
\begin{aligned}
\zeta \notin \mathrm{WF}_{\mathrm{Sc}}(u) \cap{ }^{\mathrm{sc}} T_{C_{a}^{\prime}}^{*}\left(C_{a} ; X\right) \quad \text { iff } \quad & \exists A \in \Psi_{\mathrm{Sc}}^{0,0}(X, \mathcal{C}), \hat{A}_{a, 0}(\zeta) \text { invertible in } \Psi_{\mathrm{Sc}}^{0,0}\left(\bar{X}^{a}, \mathcal{C}^{a}\right), \\
& \exists B_{j} \in \Psi_{\mathrm{Sc}}^{-\infty, 0}(X, \mathcal{C}), \zeta \notin \mathrm{WF}_{\mathrm{Sc}}^{\prime}\left(B_{j}\right), \\
& \exists u_{j} \in \mathcal{C}^{-\infty}(X), j=1, \ldots, s, f \in \dot{\mathcal{C}}^{\infty}(X), \\
& A u=\sum_{j=1}^{s} B_{j} u_{j}+f .
\end{aligned}
$$

Here we used the Euclidean notation $\Psi_{\mathrm{Sc}}^{0,0}\left(\bar{X}^{a}, \mathcal{C}^{a}\right)$ instead of $\Psi_{\mathrm{Sc}}^{0,0}\left(\rho_{a}^{-1}(p), T_{p} \mathcal{C}^{a}\right)$ for the sake of simplicity. Similarly, the filtered version of the Sc-wave front set is given by

$$
\begin{aligned}
& \zeta \notin \mathrm{WF}_{\mathrm{Sc}}^{m, l}(u) \cap{ }^{\mathrm{sc}} T_{C_{a}^{\prime}}^{*}\left(C_{a} ; X\right) \quad \text { iff } \quad \exists A \in \Psi_{\mathrm{Sc}}^{0,0}(X, \mathcal{C}), \hat{A}_{a, 0}(\zeta) \text { invertible in } \Psi_{\mathrm{Sc}}^{0,0}\left(\bar{X}^{a}, \mathcal{C}^{a}\right), \\
& \exists B_{j} \in \Psi_{\mathrm{Sc}}^{-\infty, 0}(X, \mathcal{C}), \zeta \notin \mathrm{WF}_{\mathrm{Sc}}^{\prime}\left(B_{j}\right), \\
& \exists u_{j} \in \mathcal{C}^{-\infty}(X), j=1, \ldots, s, f \in H_{\mathrm{sc}}^{m, l}(X), \\
& A u=\sum_{j=1}^{s} B_{j} u_{j}+f .
\end{aligned}
$$

Thus, if $p \in C_{a}^{\prime}$, then the part of $\mathrm{WF}_{\mathrm{Sc}}$ over $p$ lives in ${ }^{\mathrm{sc}} T_{p}^{*}\left(C_{a} ; X\right)$. If we define the scattering wave front set, $\mathrm{WF}_{\mathrm{sc}}(u)$, in terms of operators instead of the description of $\mathrm{WF}_{\mathrm{sc}}(u)$ given in 
(5.8) then the extra terms $B_{j} u_{j}$ can be dropped. In fact, (5.8) is equivalent to requiring that $A u \in \dot{\mathcal{C}}^{\infty}\left(\mathbb{S}_{+}^{n}\right)$ where $A=\mathcal{F}^{-1} \phi \mathcal{F} \psi \in \Psi_{\mathrm{sc}}^{-\infty, 0}\left(\mathbb{S}_{+}^{n}\right), \psi$ as above, and $\phi \in \mathcal{C}_{c}^{\infty}\left(\mathbb{R}^{n}\right)$ is identically 1 near $\xi$. The additional terms $B_{j} u_{j}$ for $\mathrm{WF}_{\mathrm{Sc}}(u)$ thus arise because the invertibility of $\hat{A}_{a}(\zeta)$ implies that $\sigma_{\mathrm{Sc}, 0}\left(\hat{A}_{a}(\zeta)\right)$ cannot vanish which in turn means that $\sigma_{\mathrm{Sc}, 0}\left(\hat{A}_{a}\left(\zeta^{\prime}\right)\right)$ is non-zero for every $\zeta^{\prime} \in{ }^{\mathrm{sc}} T_{p}^{*}\left(C_{a} ; X\right)$ since $\sigma_{\mathrm{Sc}, 0}\left(\hat{A}_{a}(\zeta)\right)=\sigma_{\mathrm{Sc}, 0}\left(\hat{A}_{a}\left(\zeta^{\prime}\right)\right)$. This simply corresponds to the conic cutoff requirement discussed before the definition.

Remark 5.3. - We remark that if $u=P u^{\prime}$ for some $u^{\prime} \in \mathcal{C}^{-\infty}(X), P \in \Psi_{\mathrm{Sc}}^{-\infty, 0}(X, \mathcal{C})$, then the following is a sufficient condition for $\zeta=\left(p, \xi_{a}\right) \in{ }^{\mathrm{sc}} T_{C_{a}^{\prime}}^{*} \bar{X}_{a}$, considered as $C_{a}^{\prime} \times X_{a}$, not to be in $\mathrm{WF}_{\mathrm{Sc}}(u)$. Suppose that there exists $\psi \in \mathcal{C}^{\infty}\left(\mathbb{S}_{+}^{n}\right), \psi(p) \neq 0$, and $\rho \in \mathcal{C}_{c}^{\infty}\left(X_{a}\right), \rho \equiv 1$ near $\xi_{a}$, and $\left(\left(\pi^{a}\right)^{*} \rho\right) \mathcal{F}(\psi u) \in \mathcal{S}\left(\mathbb{R}^{n}\right)=\mathcal{S}\left(X_{0}\right)$. Then $\zeta \notin \mathrm{WF}_{\mathrm{Sc}}(u)$. Indeed, let

$$
B=\left(\operatorname{Id}-\mathcal{F}^{-1}\left(\left(\pi^{a}\right)^{*} \rho\right) \mathcal{F} \psi\right) P, \quad v=u^{\prime}, \quad f=\mathcal{F}^{-1}\left(\left(\pi^{a}\right)^{*} \rho\right) \mathcal{F} \psi P u^{\prime} .
$$

Then $B \in \Psi_{\mathrm{Sc}}^{-\infty, 0}(X, \mathcal{C})$ (see the beginning of Section 9, in particular Lemma 9.1, for a similar construction),

$$
u=P u^{\prime}=\left(\operatorname{Id}-\mathcal{F}^{-1}\left(\left(\pi^{a}\right)^{*} \rho\right) \mathcal{F} \psi\right) P u^{\prime}+f=B v+f,
$$

$\zeta \notin \mathrm{WF}_{\mathrm{Sc}}^{\prime}(B), f \in \dot{\mathcal{C}}^{\infty}\left(\mathbb{S}_{+}^{n}\right)$. Such a characterization is useful for approximate generalized eigenfunctions $u$ of a many-body Hamiltonian $H$; see also Proposition 5.5.

With the topology we put on ${ }^{\mathrm{sc}} \dot{T}^{*} X, \mathrm{WF}_{\mathrm{Sc}}(u)$ is closed due to the relationship between the indicial operators mentioned above. Namely, the invertibility of $\hat{A}_{a, 0}(\zeta)$ implies that of $\hat{A}_{b, 0}(\tilde{\zeta})$ with $\tilde{\pi}_{b a}(\tilde{\zeta})=\zeta$, hence of $\hat{A}_{b, 0}\left(\tilde{\zeta}^{\prime}\right)$ for nearby $\tilde{\zeta}^{\prime}$. As the complement of $\mathrm{WF}_{\mathrm{Sc}}^{\prime}\left(B_{j}\right)$ is open, this implies that the complement of $\mathrm{WF}_{\mathrm{Sc}}(u)$ is also open.

In addition, $\mathrm{WF}_{\mathrm{Sc}}$ has the standard properties one would expect from a wave front set. However, only (5.16) plays an important role in our positive commutator proofs, so we refer to [19, Section 7] for detailed arguments; we only need simple modifications of the proofs presented there. Thus,

$$
\mathrm{WF}_{\mathrm{Sc}}\left(u_{1}+u_{2}\right) \subset \mathrm{WF}_{\mathrm{Sc}}\left(u_{1}\right) \cup \mathrm{WF}_{\mathrm{Sc}}\left(u_{2}\right)
$$

and the corresponding result also holds for the filtered wave front set. Moreover, pseudodifferential operators are microlocal in the sense that

$$
A \in \Psi_{\mathrm{Sc}}^{m, l}(X, \mathcal{C}), u \in \mathcal{C}^{-\infty}(X) \Rightarrow \mathrm{WF}_{\mathrm{Sc}}(A u) \subset \mathrm{WF}_{\mathrm{Sc}}(u)
$$

and similarly

$$
A \in \Psi_{\mathrm{Sc}}^{m, l}(X, \mathcal{C}), u \in \mathcal{C}^{-\infty}(X) \Rightarrow \mathrm{WF}_{\mathrm{Sc}}^{m^{\prime}-m, l+l^{\prime}}(A u) \subset \mathrm{WF}_{\mathrm{Sc}}^{m^{\prime}, l^{\prime}}(u) .
$$

We also refer to the remarks after Proposition 5.5 for connecting this wave front set to the one discussed in [40] in three-body scattering.

This wave front set, $\mathrm{WF}_{\mathrm{Sc}}$, gives a complete microlocal description of distributions at $\partial X$. To state it generally, we would need to define the extension of the standard wave front set of $u$ to give a subset of ${ }^{\mathrm{Sc}} S^{*}[X ; \mathcal{C}]$, but for us the following extension of (5.14) suffices.

PROPOSITION 5.4.-

$$
\begin{aligned}
& P \in \Psi_{\mathrm{Sc}}^{-\infty, l}(X, \mathcal{C}), \quad \mathrm{WF}_{\mathrm{Sc}}^{\prime}(P) \text { compact } \\
& u \in \mathcal{C}^{-\infty}(X), \quad \mathrm{WF}_{\mathrm{Sc}}^{\prime}(P) \cap \mathrm{WF}_{\mathrm{Sc}}(u)=\emptyset \Rightarrow P u \in \dot{\mathcal{C}}^{\infty}(X) .
\end{aligned}
$$


We remark that in [22], $\mathrm{WF}_{\mathrm{sc}}(u)$ (or rather its part over $\partial X$ ) is defined as a subset of ${ }^{\mathrm{sc}} \bar{T}_{\partial X}^{*} X$, the radial compactification of ${ }^{\mathrm{sc}} T_{\partial X}^{*} X$ in the fibers. The part at fiber-infinity, i.e. at the boundary arising from the radial compactification of the fibers, extends the usual wave front set from the interior. However, for us this extension is not important; the operator wave front set of nearly all operators we are interested in is contained in a compact region of ${ }^{\mathrm{sc}} \dot{T}^{*} X$.

Since (5.16) is the main property of the wave front set that we need for the positive commutator estimates, we briefly outline its proof.

Proof. - Suppose $\zeta \notin \mathrm{WF}_{\mathrm{Sc}}(u)$. Then there exist $A_{\zeta}, B_{\zeta, j}, u_{\zeta, j}, f_{\zeta}$, as in Definition 5.2. Let $G_{\zeta}$ be a microlocal parametrix for $A_{\zeta}$, so Id $=G_{\zeta} A_{\zeta}+R_{\zeta}$, with $G_{\zeta}, R_{\zeta} \in \Psi_{\mathrm{Sc}}^{0,0}(X, \mathcal{C})$, $\zeta \notin \mathrm{WF}_{\mathrm{Sc}}^{\prime}\left(R_{\zeta}\right)$. Then

$$
u=G_{\zeta} A_{\zeta} u+R_{\zeta} u=\sum_{j} G_{\zeta} B_{\zeta, j} u_{\zeta, j}+G_{\zeta} f_{\zeta}+R_{\zeta} u .
$$

Now, $U_{\zeta}=\left(\mathrm{WF}_{\mathrm{Sc}}^{\prime}\left(R_{\zeta}\right) \cup\left(\bigcup_{j} \mathrm{WF}_{\mathrm{Sc}}^{\prime}\left(B_{\zeta, j}\right)\right)\right)^{c}$ is open, and $\left\{U_{\zeta}: \zeta \notin \mathrm{WF}_{\mathrm{Sc}}(u)\right\}$ is a cover of the complement of $\mathrm{WF}_{\mathrm{Sc}}(u)$, hence in particular of $\mathrm{WF}_{\mathrm{Sc}}^{\prime}(P)$. Since the latter is compact, there is a finite subcover, say $\left\{U_{\zeta_{k}}: k=1, \ldots, N\right\}$.

Using a partition of unity, we can write $P=\sum_{k} P_{k}$ with $P_{k} \in \Psi_{\mathrm{Sc}}^{-\infty, 0}(X, \mathcal{C}), \mathrm{WF}_{\mathrm{Sc}}^{\prime}\left(P_{k}\right) \subset$ $U_{\zeta_{k}}$. Indeed, as discussed before Definition 5.1, we can find $\pi$-invariant functions $q_{k} \in$ $\mathcal{C}^{\infty}\left({ }^{\mathrm{sc}} T_{\partial X}^{*} X\right)$ with $\operatorname{supp}\left(q_{k}\right)_{\pi} \cap \mathrm{WF}_{\mathrm{Sc}}^{\prime}(P) \subset U_{\zeta_{k}}$ and $q_{0}=1-\sum_{k} q_{k}$ vanishes identically on a neighborhood of $\mathrm{WF}_{\mathrm{Sc}}^{\prime}(P)$. Quantizing these as in Section 9, i.e. quantizing $q_{k} p$ where $P$ is the right quantization of $p$, see Lemma 9.1, using $P \in \Psi_{\mathrm{Sc}}^{-\infty, 0}(X, \mathcal{C})$ in place of $\psi_{0}(H)$, gives operators $P_{k} \in \Psi_{\mathrm{Sc}}^{-\infty, 0}(X, \mathcal{C})$ with $\mathrm{WF}_{\mathrm{Sc}}^{\prime}\left(P_{k}\right) \subset U_{\zeta_{k}}$ for $k \geqslant 1, P_{0} \in \Psi_{\mathrm{Sc}}^{-\infty, \infty}(X, \mathcal{C})$, and $\sum_{k=0}^{N} P_{k}=P$. Since $P_{0}$ has empty operator wave front set, it can be added to any of the other $P_{k}$; henceforth we drop it from our notation. Then, with $G_{k}=G_{\zeta_{k}}$, etc.,

$$
P_{k} u=\sum_{j} P_{k} G_{k} B_{k, j} u_{k, j}+P_{k} G_{k} f_{k}+P_{k} R_{k} u
$$

Since $P_{k} \in \Psi_{\mathrm{Sc}}^{-\infty, 0}(X, \mathcal{C})$, all composite operators on the right hand side are also in $\Psi_{\mathrm{Sc}}^{-\infty, 0}(X, \mathcal{C})$. Moreover, by construction, $\mathrm{WF}_{\mathrm{Sc}}^{\prime}\left(P_{k}\right) \cap \mathrm{WF}_{\mathrm{Sc}}^{\prime}\left(B_{k, j}\right)=\emptyset, \mathrm{WF}_{\mathrm{Sc}}^{\prime}\left(P_{k}\right) \cap \mathrm{WF}_{\mathrm{Sc}}^{\prime}\left(R_{k}\right)=\emptyset$, so $P_{k} G_{k} B_{k, j}$ and $P_{k} R_{k}$ are both in $\Psi_{\mathrm{Sc}}^{-\infty, \infty}(X, \mathcal{C})$, hence map $\mathcal{C}^{-\infty}(X)$ to $\dot{\mathcal{C}}^{\infty}(X)$, while $f_{k} \in \dot{\mathcal{C}}^{\infty}(X)$, so $P_{k} G_{k} f_{k} \in \dot{\mathcal{C}}^{\infty}(X)$ as well. Thus, $P_{k} u \in \dot{\mathcal{C}}^{\infty}(X)$, which in turn implies $P u \in \dot{\mathcal{C}}^{\infty}(X)$ as claimed.

The description of the wave front set becomes simpler for generalized eigenfunctions of manybody Hamiltonians $H$. Namely, we have the following result.

Proposition 5.5. - Suppose that $u \in \mathcal{C}^{-\infty}(X), H \in \Psi_{\mathrm{Sc}}^{m, 0}(X, \mathcal{C}), m>0$ is self-adjoint and $\sigma_{\mathrm{Sc}, m}(H)$ never vanishes. Let $\lambda \in \mathbb{R}$, and define $W \subset{ }^{\mathrm{sc}} \dot{T}^{*} X$ by

$$
\begin{aligned}
\zeta \notin W \cap{ }^{\mathrm{sc}} T_{C_{a}^{\prime}}^{*}\left(C_{a} ; X\right) \quad \text { iff } \quad & \exists \psi \in \mathcal{C}_{c}^{\infty}(\mathbb{R}), \psi(\lambda)=1, \\
& \exists A \in \Psi_{\mathrm{Sc}}^{-\infty, 0}(X, \mathcal{C}), \hat{A}_{a}(\zeta)=\widehat{\psi(H)}, A u \in \dot{\mathcal{C}}^{\infty}(X) .
\end{aligned}
$$

Then

$$
\mathrm{WF}_{\mathrm{Sc}}(u) \subset \mathrm{WF}_{\mathrm{Sc}}((H-\lambda) u) \cup W .
$$

The same conclusion holds with $\mathrm{WF}_{\mathrm{Sc}}$ replaced by $\mathrm{WF}_{\mathrm{Sc}}^{m, l}$ and $A u \in \dot{\mathcal{C}}^{\infty}(X)$ by $A u \in H_{\mathrm{sc}}^{m, l}(X)$. 
Proof. - Suppose that $\zeta \notin \mathrm{WF}_{\mathrm{Sc}}((H-\lambda) u)$ and $\zeta \notin W$. With $\psi$ as above, let $\tilde{\psi}(t)=$ $(1-\psi(t)) /(t-\lambda)$, so $\tilde{\psi} \in S_{\mathrm{phg}}^{-1}(\mathbb{R})$ as $\psi(\lambda)=1$. Then $\tilde{\psi}(H) \in \Psi_{\mathrm{Sc}}^{-m, 0}(X, \mathcal{C})$ and Id $=$ $\tilde{\psi}(H)(H-\lambda)+\psi(H)$. With $A$ as above, let $A^{\prime}=A+(\operatorname{Id}-\psi(H)) \in \Psi_{\mathrm{Sc}}^{0,0}(X, \mathcal{C})$. Then $\hat{A}_{a}^{\prime}(\zeta)=\operatorname{Id}$ and

$$
A^{\prime} u=A u+\tilde{\psi}(H)(H-\lambda) u .
$$

But $A u \in \dot{\mathcal{C}}^{\infty}(X)$ by assumption, so by (5.14)

$$
\mathrm{WF}_{\mathrm{Sc}}\left(A^{\prime} u\right)=\mathrm{WF}_{\mathrm{Sc}}(\tilde{\psi}(H)(H-\lambda) u) \subset \mathrm{WF}_{\mathrm{Sc}}((H-\lambda) u) .
$$

Hence, there exist $A^{\prime \prime}$ (in place of $A$ ), $B_{j}$, etc., as in Definition 5.2, $A^{\prime \prime} A^{\prime} u=f+\sum B_{j} u_{j}$, and the indicial operator of $A^{\prime \prime} A^{\prime}$ at $\zeta$ is just the composite of those of $A^{\prime \prime}$ and $A^{\prime}$, hence invertible, showing that $\zeta \notin \mathrm{WF}_{\mathrm{Sc}}(u)$.

Remark 5.6. - Our definition of $\mathrm{WF}_{\mathrm{Sc}}(u)$, which is in particular valid if $(X, \mathcal{C})$ is a threebody space, is different from the wave front set $\mathrm{WF}_{3 s c}(u)$ used in [40] in the three-body setting. Indeed, in the definition of $\mathrm{WF}_{3 s c}(u)$, the terms $B_{j} u_{j}$ appearing in Definition 5.2 were not allowed. Consequently, (5.14), and its filtered analogue did not hold in general. However, for the positive commutator proofs of both [40] and the present paper, one only needs (5.16), which was proved for $\mathrm{WF}_{3 s c}$. Note that $\mathrm{WF}_{3 s c}^{\prime}(A)$ and $\mathrm{WF}_{\mathrm{Sc}}^{\prime}(A)$ are compact for all operators appearing in positive commutator estimates in both papers.

Note that $\mathrm{WF}_{\mathrm{Sc}}(u) \subset \mathrm{WF}_{3 s c}(u)$ directly from the definition. Moreover, if $(\operatorname{Id}-P) u \in$ $\dot{\mathcal{C}}^{\infty}(X)$ for some $P \in \Psi_{\mathrm{Sc}}^{-\infty, 0}(X, \mathcal{C})$ (e.g. $P=\psi(H)$ in the setting of the proposition) then $\mathrm{WF}_{\mathrm{Sc}}(u)=\mathrm{WF}_{3 s c}(u)$. In fact, suppose that $\zeta \notin \mathrm{WF}_{\mathrm{Sc}}(u)$, so $A u=\sum B_{j} u_{j}+f$ as in Definition 5.2. Since $\hat{A}$ is invertible near $\zeta$, we can arrange (by inverting $\hat{A}$ nearby, i.e. by constructing a 'microlocal parametrix') that $u=\sum B_{j}^{\prime} u_{j}+f^{\prime}$ with $B_{j}^{\prime} \in \Psi_{\mathrm{Sc}}^{-\infty, 0}(X, \mathcal{C})$, $\zeta \notin \mathrm{WF}_{\mathrm{Sc}}^{\prime}\left(B_{j}^{\prime}\right)$ (cf. [19, Lemma 16]; we remove the term $C u$ stated there by writing it as $C P u+C(\operatorname{Id}-P) u$, and incorporating them in $\sum B_{j}^{\prime} u_{j}^{\prime}$ and $f^{\prime}$ respectively). Using the methods of Section 9, given any neighborhood $U$ of $\zeta$, it is easy to construct an operator $G \in \Psi_{\mathrm{Sc}}^{-\infty, 0}(X, \mathcal{C})$ such that $\mathrm{WF}_{\mathrm{Sc}}^{\prime}(G) \subset U$ and $\zeta \notin \mathrm{WF}_{\mathrm{Sc}}^{\prime}(P-G)$ (hence the same holds for a neighborhood of $\zeta)$. Since the indicial operator of $Q=G+(\operatorname{Id}-P)$ at $\zeta$ is the identity, and since $(\operatorname{Id}-P) u \in \dot{\mathcal{C}}^{\infty}(X)$, we only need to prove that $G u \in \dot{\mathcal{C}}^{\infty}(X)$ to conclude that $\zeta \notin \mathrm{WF}_{3 s c}(u)$. But $G u=\sum G B_{j}^{\prime} u_{j}+G f^{\prime}$, so if $U$ is chosen sufficiently small, then $G B_{j}^{\prime} \in \Psi_{\mathrm{Sc}}^{-\infty, \infty}(X, \mathcal{C})$, so $G u \in \dot{\mathcal{C}}^{\infty}(X)$ indeed.

\section{The Hamiltonian and generalized broken bicharacteristics}

We next analyze the operator $H-\lambda$ where $H=\Delta+V$ and $\Delta$ is the Laplacian of a scattering metric

$$
g=\frac{d x^{2}}{x^{4}}+\frac{h^{\prime}}{x^{2}}
$$

Recall that $h^{\prime}$ is a smooth symmetric 2-cotensor on $X$ whose restriction to $\partial X$ (i.e. its pull-back), $h$, is positive definite. We assume that

$$
V \in \mathcal{C}^{\infty}([X ; \mathcal{C}] ; \mathbb{R}) \text { vanishes at } \beta_{\mathrm{Sc}}^{*} C_{0},
$$

i.e. $V$ vanishes in the free region. This implies that

$$
H \in \operatorname{Diff}_{\mathrm{Sc}}^{2}(X, \mathcal{C}) .
$$


Such a situation arises, for example, in actual Euclidean scattering if the potentials $V_{a}$ (in the notation of the introduction) are classical symbols of order -1 on $X^{a}$. Hence, we make the following definition.

Definition 6.1. - A many-body Hamiltonian is an operator $H=\Delta+V$ where $\Delta$ is the Laplacian of a scattering metric $g$, and $V$ satisfies (6.2).

As indicated in the Introduction, from this point on we also make the assumption

$$
(X, \mathcal{C}) \text { is locally linearizable; }
$$

this will simplify the analysis. We recall that this is equivalent to the local existence of Riemannian metrics on $\partial X$, possibly different from $h$, with respect to which all elements of $\mathcal{C}$ are totally geodesic.

Since $\sigma_{\mathrm{sc}, 2}(\Delta)$ never vanishes, the same holds for $\sigma_{\mathrm{Sc}, 2}(H)$ which is the pull-back of the former. A simple calculation, see [40, Sections 4 and 11] for more details, shows that the indicial operators of $H$ are given by

$$
\begin{gathered}
\hat{H}_{a, 0}(\xi)=\hat{H}_{a, 0}((p, 0))+\tau^{2}+\tilde{h}(z, \nu), \quad \xi=(z, \tau, \nu) \in{ }^{\mathrm{sc}} T^{*}\left(\tilde{C}_{a} ; X\right), \\
\hat{H}_{a, 0}(p, 0)=\Delta_{Y}+V(p, Y)
\end{gathered}
$$

where $Y$ are 'Euclidean coordinates' on the interior of $\rho_{a}^{-1}(p)$, i.e. that of $\tilde{\beta}_{a}^{-1}(p)$, and $\Delta_{Y}$ is the Euclidean Laplacian.

More precisely, we have seen in Section 4 that $\left(\beta\left[X ; \mathcal{C}_{a}\right]_{p}^{* \mathrm{sc}} T X\right) /{ }^{\mathrm{sc}} T_{p}\left(\tilde{C}_{a} ; X\right)$ naturally acts transitively and freely on the interior of $\rho_{a}^{-1}(p)=S^{+} N_{p} \tilde{C}_{a}$, so it makes sense to talk about translation invariant vector fields and differential operators on the interior of $S^{+} N_{p} \tilde{C}_{a}$. Indeed, the restriction to $S^{+} N \tilde{C}_{a}$ of the lift of elements of $\operatorname{Diff}_{\mathrm{sc}}(X)$ (under $\beta_{\mathrm{Sc}}$ ) are such. We can see this since $\mathcal{V}_{\mathrm{sc}}(X)$ is given by sections of ${ }^{\mathrm{sc}} T X$; the restriction of the lift of $P \in \mathcal{V}_{\mathrm{sc}}(X)$ is then given by the identification of $\left(\beta\left[X ; \mathcal{C}_{a}\right]_{p}^{* \mathrm{sc}} T X\right) /{ }^{\mathrm{sc}} T_{p}\left(\tilde{C}_{a} ; X\right)$ with the tangent space at each point of the fiber $\rho_{a}^{-1}(p)$. Using the metric $g$ to identify the quotient bundle with the orthocomplement of ${ }^{\mathrm{sc}} T_{p}\left(\tilde{C}_{a} ; X\right), S^{+} N_{p} \tilde{C}_{a}$ becomes an affine space with a translation-invariant metric (i.e. 'Euclidean') with the metric induced by $g ; \Delta_{Y}$ is the Laplacian of this metric.

Eqs. (6.5)-(6.6) show that $\hat{H}_{a, 0}(p, 0)$ is uniformly bounded below, so for any $\psi \in \mathcal{C}_{c}^{\infty}(\mathbb{R})$ the set

$$
\bigcup_{a} \operatorname{cl}\left(\left\{\xi \in{ }^{\mathrm{sc}} T^{*}\left(\tilde{C}_{a} ; X\right): \psi\left(\hat{H}_{a}(\xi)\right) \neq 0\right\}\right)
$$

is compact.

The bound states of the subsystems of $H$ play an important role in Euclidean many-body scattering. The appropriate replacement in the general geometric setting is given via the indicial operators of $H$. Thus, in this paper the statement 'no subsystem of $H$ has a bound state' means that

$$
\hat{H}_{a, 0}(\xi) \text { has no } L^{2} \text { eigenvalues for any } a \neq 0 \text { and } \xi \in{ }^{\mathrm{sc}} T^{*}\left(\tilde{C}_{a} ; X\right) .
$$

Due to (6.5)-(6.6), this means simply that

$$
h_{a}(p)=\hat{H}_{a, 0}((p, 0)) \text { has no } L^{2} \text { eigenvalues for any } a \neq 0 \text { and } p \in \tilde{C}_{a} .
$$


In Euclidean scattering $h_{a}(p)$ is just the subsystem Hamiltonian $h_{a}$ (which is then independent of $p$ ), so in that setting (6.8) indeed means that the (proper) subsystems of $H$ have no bound states.

If no subsystem of $H$ has bound states it can be expected that $\Delta-\lambda$ governs the propagation of singularities of distributions $u$ with $(H-\lambda) u \in \dot{\mathcal{C}}^{\infty}(X)$, except that the flow will break at the places where $V$ is singular (i.e. where locally $V \notin \mathcal{C}^{\infty}(X)$ ), similarly to boundary and transmission problems for the wave equation [12, Chapter XXIV], [23,18]. Now, the symbol of $\Delta-\lambda$ at $\partial X$ (i.e. its sc-indicial operator) is $g-\lambda$. Hence, its characteristic variety is

$$
\Sigma=\Sigma_{\Delta-\lambda}=\left\{\xi \in{ }^{\mathrm{sc}} T_{\partial X}^{*} X: g(\xi)-\lambda=0\right\} .
$$

The rescaled Hamilton vector field ${ }^{\mathrm{sc}} H_{g}=x^{-1} H_{g}$ of $g$ (or $\left.g-\lambda\right)$, introduced in [22], is

$$
{ }^{\mathrm{sc}} H_{g}=2 \tau\left(x \partial_{x}+\mu \cdot \partial_{\mu}+\nu \cdot \partial_{\nu}\right)-2 h \partial_{\tau}+H_{h}+x W^{\prime}, \quad W^{\prime} \in \mathcal{V}_{\mathrm{b}}\left({ }^{\mathrm{sc}} T^{*} X\right),
$$

so its restriction to $\partial X$, also denoted by ${ }^{\mathrm{sc}} H_{g}$, is

$$
{ }^{\mathrm{sc}} H_{g}=2 \tau\left(\mu \cdot \partial_{\mu}+\nu \cdot \partial_{\nu}\right)-2 h \partial_{\tau}+H_{h} .
$$

Here $(y, z, \tau, \mu, \nu)$ denote coordinates about some $C=C_{a}$ as before, though notice that $\mu \cdot \partial_{\mu}+\nu \cdot \partial_{\nu}$ is simply the radial vector field in $T^{*} \partial X$, so the above expression is indeed invariant (as it must be). The bicharacteristics of $\Delta-\lambda$ are just integral curves of ${ }^{\mathrm{sc}} H_{g}$.

We divide the image $\dot{\Sigma} \subset{ }^{\mathrm{sc}} \dot{T}^{*} X$ of $\Sigma$ under $\pi$ into a normal and a tangential part,

$$
\dot{\Sigma}=\Sigma_{n}(\lambda) \cup \Sigma_{t}(\lambda)
$$

as follows. Let $\hat{\pi}$ be the restriction of $\pi$ to $\Sigma$. We let

$$
\Sigma_{n}(\lambda)=\bigcup_{a}\left\{\xi \in{ }^{\mathrm{sc}} T_{C_{a}^{\prime}}^{*}\left(C_{a} ; X\right) \cap \dot{\Sigma}: \hat{\pi}^{-1}(\xi) \text { consists of more than one point }\right\}
$$

and

$$
\Sigma_{t}(\lambda)=\bigcup_{a}\left\{\xi \in{ }^{\mathrm{sc}} T_{C_{a}^{\prime}}^{*}\left(C_{a} ; X\right) \cap \dot{\Sigma}: \hat{\pi}^{-1}(\xi) \text { consists of exactly one point }\right\} .
$$

In terms of our local coordinates around $C_{a}^{\prime}$, in view of (4.23) and $\left|\mu_{a}\right|^{2} \geqslant 0$, this means that

$$
\Sigma_{n}(\lambda)=\bigcup_{a}\left\{\left(z_{a}, \tau_{a}, \nu_{a}\right) \in{ }^{\mathrm{sc}} T_{C_{a}^{\prime}}^{*}\left(C_{a} ; X\right): \tau_{a}^{2}+\tilde{h}\left(z_{a}, \nu_{a}\right)<\lambda\right\}
$$

and

$$
\Sigma_{t}(\lambda)=\bigcup_{a}\left\{\left(z_{a}, \tau_{a}, \nu_{a}\right) \in{ }^{\mathrm{sc}} T_{C_{a}^{\prime}}^{*}\left(C_{a} ; X\right): \tau_{a}^{2}+\tilde{h}\left(z_{a}, \nu_{a}\right)=\lambda\right\} .
$$

Notice that for $\xi=\left(z_{a}, \tau_{a}, \nu_{a}\right) \in \Sigma_{t}(\lambda)$ and the unique point $\tilde{\xi}=\left(0, z_{a}, \tau_{a}, \mu_{a}, \nu_{a}\right) \in{ }^{\mathrm{sc}} T_{\partial X}^{*} X$ with $\pi(\tilde{\xi})=\xi$ we have $\mu_{a}=0$. As the $\partial_{y_{a}}$ component of ${ }^{\mathrm{sc}} H_{g}$ is $2 \mu_{a} \cdot \partial_{y_{a}}$ at $y_{a}=0$ (i.e. at $C_{a}$ ), for such $\xi$ and $\tilde{\xi},{ }^{\mathrm{sc}} H_{g}(\tilde{\xi})$ is tangent to ${ }^{\mathrm{sc}} T_{C_{a}^{\prime}}^{*} X$. On the other hand, if $\xi \in \Sigma_{n}(\lambda), \tilde{\xi} \in \hat{\pi}^{-1}(\xi)$, then ${ }^{\mathrm{sc}} H_{g}(\tilde{\xi})$ is normal to ${ }^{\mathrm{sc}} T_{C_{a}^{\prime}}^{*} X$, hence the choice of our terminology. Notice also that on ${ }^{\mathrm{sc}} T_{C_{0}^{\prime}}^{*} X, \pi$ is the identity map, so

$$
\dot{\Sigma} \cap{ }^{\mathrm{sc}} T_{C_{0}^{\prime}}^{*} X \subset \Sigma_{t}(\lambda)
$$

4e SÉRIE - TOME $34-2001-\mathrm{N}^{\circ} 3$ 
We also define the radial sets $R_{ \pm}(\lambda)$ as the sets

$$
R_{ \pm}(\lambda)=\pi(\{(y, z, \tau, \mu, \nu): \tau= \pm \sqrt{\lambda}, h(y, z, \mu, \nu)=0\})
$$

Thus, $R_{+}(\lambda) \cup R_{-}(\lambda)$ is the image (under $\pi$ ) of the set where ${ }^{\mathrm{sc}} H_{g}$ vanishes. Notice that

$$
R_{+}(\lambda) \cup R_{-}(\lambda) \subset \Sigma_{t}(\lambda) .
$$

Following Lebeau, we define generalized broken bicharacteristics of $\Delta-\lambda$ as follows. First, recall from Section 5 that we say that a function $f \in \mathcal{C}^{\infty}\left({ }^{\mathrm{sc}} T_{\partial X}^{*} X\right)$ is $\pi$-invariant if for $\tilde{\xi}, \tilde{\xi}^{\prime} \in{ }^{\mathrm{sc}} T_{\partial X}^{*} X, \pi(\tilde{\xi})=\pi\left(\tilde{\xi}^{\prime}\right)$ implies $f(\tilde{\xi})=f\left(\tilde{\xi}^{\prime}\right)$. A $\pi$-invariant function $f$ naturally defines a function $f_{\pi}$ on ${ }^{\mathrm{sc}} \dot{T}^{*} X$ by $f_{\pi}(\xi)=f(\tilde{\xi})$ where $\tilde{\xi} \in{ }^{\mathrm{sc}} T_{\partial X}^{*} X$ is chosen so that $\pi(\tilde{\xi})=\xi$.

Definition 6.2. - Suppose that $(X, \mathcal{C})$ is locally linearizable. A generalized broken bicharacteristic of $\Delta-\lambda$ is a continuous map $\gamma: I \rightarrow{ }^{\text {sc }} \dot{T}^{*} X$, where $I \subset \mathbb{R}$ is an interval, satisfying the following requirements:

(i) If $\xi_{0}=\gamma\left(t_{0}\right) \in \Sigma_{t}(\lambda)$ then for all $\pi$-invariant functions $f \in \mathcal{C}^{\infty}\left({ }^{\mathrm{sc}} T_{\partial X}^{*} X\right)$,

$$
\frac{d}{d t}\left(f_{\pi} \circ \gamma\right)\left(t_{0}\right)={ }^{\mathrm{sc}} H_{g} f\left(\tilde{\xi}_{0}\right), \quad \tilde{\xi}_{0}=\hat{\pi}^{-1}\left(\xi_{0}\right) .
$$

(ii) If $\xi_{0}=\gamma\left(t_{0}\right) \in \Sigma_{n}(\lambda) \cap{ }^{\mathrm{sc}} T_{C_{a}^{\prime}}^{*}\left(C_{a} ; X\right)$ then there exists $\varepsilon>0$ such that

$$
t \in I, \quad 0<\left|t-t_{0}\right|<\varepsilon \Rightarrow \gamma(t) \notin{ }^{\mathrm{sc}} T_{C_{a}^{\prime}}^{*}\left(C_{a} ; X\right) .
$$

The success of this definition (so that it indeed describes what we wish to describe) depends on a plentiful supply of $\pi$-invariant functions on ${ }^{\mathrm{sc}} T_{\partial X}^{*} X$. Under our local linearizability hypothesis, (6.4), there are always many such functions. Recall from Section 5 that by (6.4), if $p \in C^{\prime}=C_{a}^{\prime}$, we can choose local coordinates $(y, z)$ on $\partial X$ in terms of which all the $C_{b}$ satisfying $p \in C_{b}$ are linear, i.e. they are given by $A_{b} y=0$ where $A_{b}$ is a (constant) matrix, and $C_{a}$ is given by $y=0$. With $(\tau, \mu, \nu)$ denoting the sc-dual variables of $(x, y, z)$, we see that $y, z, \tau, \nu$ are $\pi$-invariant near ${ }^{\mathrm{sc}} T_{p}^{*} X$. In general, without the assumption (6.4), $\nu$ would not be $\pi$-invariant, and we would not be able to modify it to make it such, so the definition would be inadequate.

We can also arrange that the metric function is of the form $h=\tilde{h}(z, \nu)+h_{n n}(z, \mu)$ at $C_{a}^{\prime}$ by a further change of coordinates $z_{j}^{\prime}=z_{j}+\sum_{j k} Z_{j k}(z) y_{k}, y^{\prime}=y$, which preserves the linear structure of the $C_{b}$. In general we cannot arrange that $h_{n n}(z, \mu)=|\mu|^{2}$ everywhere along $C_{a}^{\prime}$ without destroying the product-linear structure of the $C_{b}$. However, by a linear change in the $y$ coordinates we can make sure that at a fixed $p \in C_{a}^{\prime}, h=\tilde{h}(z, \nu)+|\mu|^{2}$. The continuity of a generalized broken bicharacteristic $\gamma$ means that if $\gamma\left(t_{0}\right) \in{ }^{\mathrm{sc}} T_{C^{\prime}}^{*}(C ; X)$, then for $t$ near $t_{0}, t \mapsto(y(\gamma(t)), z(\gamma(t)), \tau(\gamma(t)), \nu(\gamma(t)))$ is continuous, but $\mu(\gamma(t))$ may be discontinuous. In terms of Euclidean scattering this means that at $C_{a}$ the external momentum is conserved, but not necessarily the internal one, while image $(\gamma) \subset \dot{\Sigma}$ corresponds to the conservation of kinetic energy. The latter cannot be expected to hold if the subsystems of the Hamiltonian have bound states; the relevant broken bicharacteristics in that case exhibit more complex behavior. Another example of a $\pi$-invariant function in this situation is $y \cdot \mu$; this will play a rather important role in the propagation estimates. In fact, ${ }^{\mathrm{sc}} H_{g}(y \cdot \mu)\left(\tilde{\xi}_{0}\right)=2\left|\mu_{0}\right|^{2}$ if $\tilde{\xi}_{0} \in{ }^{\mathrm{sc}} T_{p}^{*} X$ is of the form $\left(0, z_{0}, \tau_{0}, \mu_{0}, \nu_{0}\right)$, so if $\pi\left(\tilde{\xi}_{0}\right) \in \Sigma_{n}(\lambda)$ and $\tilde{\xi}_{0} \in \Sigma$ then $y \cdot \mu$ is a parameter along generalized broken bicharacteristics near $\tilde{\xi}_{0}$ - see also the following proposition.

A stronger characterization of generalized broken bicharacteristics at $\Sigma_{n}(\lambda)$ follows as in Lebeau's paper. Notice that if $\gamma: I \rightarrow \dot{\Sigma}$ is continuous then the conclusion of the following 
proposition certainly implies (i) and (ii) ((ii) follows as $y_{j}=\left(y_{a}\right)_{j}$ are $\pi$-invariant), so the proposition indeed provides an alternative to our definition.

PROPOSITION 6.3 (Lebeau, [18, Proposition 1]). - If $\gamma$ is a generalized broken bicharacteristic as above, $t_{0} \in I, \xi_{0}=\gamma\left(t_{0}\right)$, then there exist unique $\tilde{\xi}_{+}, \tilde{\xi}_{-} \in \Sigma(\Delta-\lambda)$ satisfying $\pi\left(\tilde{\xi}_{ \pm}\right)=\xi_{0}$ and having the property that if $f \in \mathcal{C}^{\infty}\left({ }^{\mathrm{sc}} T_{\partial X}^{*} X\right)$ is $\pi$-invariant then $t \mapsto f_{\pi}(\gamma(t))$ is differentiable both from the left and from the right at $t_{0}$ and

$$
\left.\left(\frac{d}{d t}\right)\left(f_{\pi} \circ \gamma\right)\right|_{t_{0} \pm}={ }^{\mathrm{sc}} H_{g} f\left(\tilde{\xi}_{ \pm}\right)
$$

We refer to Lebeau's paper for the proof in the general setting, but in the Appendix we give the proof under the assumption that the elements of $\mathcal{C}$ are totally geodesic. In fact, we prove slightly more by giving a Hölder-type remainder estimate. We present the proof in the Appendix, but we emphasize that it is simply a minor modification of Lebeau's proof. We remark that the most delicate part of the conclusion (under the totally geodesic assumption) is the differentiability of the 'normal' coordinate functions $y_{j}$ along $\gamma$, i.e. that of $y_{j} \circ \gamma$. Here we dropped the projection $\pi$ from the notation (i.e. we did not write $\left.\left(y_{j}\right)_{\pi} \circ \gamma\right)$ to simplify it; we will often do this in the future for the other $\pi$-invariant coordinate functions $\tau, z_{j}, \nu_{j}$. The proof proceeds by induction using the order on $\mathcal{C}$. Thus, we have to understand what happens near $t_{0}$ if $\gamma\left(t_{0}\right)=\xi_{0} \in \Sigma_{n}(\lambda) \cap{ }^{\mathrm{sc}} T_{C^{\prime}}^{*}\left(C_{a} ; X\right)$. The inductive hypothesis is that we have already proved the proposition for $b$ with $C_{a} \subsetneq C_{b}$. Thus, by Definition 6.2, part (ii), it is true for $t_{0}$ replaced by $t \neq t_{0}$, assuming $\left|t-t_{0}\right|<\varepsilon$. Hence, we need to analyze the behavior of the coordinate functions using the Hamilton equation, (6.23) which is a little more delicate than the positive commutator construction in Proposition 10.4, but the two proofs are very closely related via the use of same function $\phi$ to localize near (and along) the generalized broken bicharacteristics. A rather similar analogy arises in our tangential estimates in the totally geodesic setting; see Propositions 7.1 and 10.6 respectively.

We now describe some corollaries of this proposition. First, we remark that the role of the globally defined $\pi$-invariant function $\tau$ is somewhat analogous to the role played by the time variable in the wave equation in Lebeau's paper. In particular, $\tau$ gives a parameter along generalized broken bicharacteristics with the exception of some trivial ones (namely the constant ones in $R_{+}(\lambda) \cup R_{-}(\lambda)$ ). To see this, we show the following corollary of the above proposition.

COROLlaRY 6.4. - Suppose that $\gamma: I \rightarrow \dot{\Sigma}$ is a generalized broken bicharacteristic. Then $T=\tau_{\pi} \circ \gamma: I \rightarrow \mathbb{R}$ is a $\mathcal{C}^{\infty}$ function. In addition, $T$ has one of the following forms. Either

(i) $T(t)=\sqrt{\lambda}$ for all $t \in I$, or

(ii) $T(t)=-\sqrt{\lambda}$ for all $t \in I$, or

(iii) $T^{\prime}(t)<0$ for all $t$ and if $I=\mathbb{R}$ then $T(t) \rightarrow \mp \sqrt{\lambda}$ as $t \rightarrow \pm \infty$.

Proof. - As $\lambda=\tau^{2}+h$ in $\Sigma_{\Delta-\lambda}$, we have for all $\tilde{\xi} \in \Sigma_{\Delta-\lambda}$ that

$$
{ }^{\mathrm{sc}} H_{g} \tau(\tilde{\xi})=-2 h(\tilde{\xi})=2\left(\tau(\tilde{\xi})^{2}-\lambda\right) .
$$

Thus, with $T=\tau_{\pi} \circ \gamma$, the previous proposition implies that for any $t \in I, T$ is differentiable from both the left and the right at $t$, and both of these derivatives are equal to $2\left(T(t)^{2}-\lambda\right)$. (We remark that this is proved directly in the Appendix as a first step to the proof of the proposition.) Thus, $T$ is $\mathcal{C}^{1}$ and it satisfies the ODE $d T / d t=2\left(T^{2}-\lambda\right)$. But, given say $T\left(t_{0}\right)=\tau_{0}$, this ODE has a unique solution which is $\mathcal{C}^{\infty}$. The last statement follows by writing down the solution of the ODE explicitly, which, if $T\left(t_{0}\right) \in(-\lambda, \lambda)$ for some $t_{0} \in I$, takes the form $T(t)=-\sqrt{\lambda} \tanh (4 \sqrt{\lambda}(t-c)), t \in I$, for an appropriate constant $c$. 
Since for $\xi \in \dot{\Sigma}$ with $\tau(\xi)^{2}=\lambda$ we automatically have $\xi \in R_{+}(\lambda) \cup R_{-}(\lambda)$, in (iii) we see that (if $I=\mathbb{R}$ ) as $t \rightarrow \pm \infty, \gamma(t)$ approaches $R_{\mp}(\lambda)$. In addition, in the same case, as $T^{\prime}$ never vanishes, $T \in(-\sqrt{\lambda}, \sqrt{\lambda})$ can be used to reparameterize $\gamma$ (reversing its direction).

We proceed to examine generalized broken bicharacteristics in more detail, starting with cases (i) and (ii). Namely, we prove that generalized broken bicharacteristics through $R_{+}(\lambda) \cup R_{-}(\lambda)$ are constant maps:

PROPOSITION 6.5.- If $\gamma: I \rightarrow \dot{\Sigma}$ is a generalized broken bicharacteristic, $\gamma\left(t_{0}\right)=\xi_{0} \in R_{+}(\lambda) \cup R_{-}(\lambda)$, then $\gamma(t)=\xi_{0}$ for $t \in I$. Hence, $\hat{\pi}^{-1} \circ \gamma$ is a bicharacteristic of ${ }^{\mathrm{sc}} H_{g}$.

Proof. - The previous corollary and the above remarks show that for all $t \in I, \gamma(t) \in R_{+}(\lambda) \cup$ $R_{-}(\lambda)$. Let $\tilde{\xi}(t)=\hat{\pi}^{-1}(\gamma(t))$. Thus, ${ }^{\text {sc }} H_{g}$ vanishes at $\tilde{\xi}(t) \in \hat{\pi}^{-1}\left(R_{+}(\lambda) \cup R_{-}(\lambda)\right)$ for all $t$. Since the base variables $y$ and $z$ are $\pi$-invariant, we conclude that $d\left(\left(y_{j}\right)_{\pi} \circ \gamma\right) / d t$ vanishes identically, hence $y$ is constant, and similarly for $z$, proving that $\gamma(t)=\xi_{0}$ for all $t$. The last statement of the proposition follows since ${ }^{\mathrm{sc}} H_{g}$ vanishes at $\hat{\pi}^{-1}\left(R_{+}(\lambda) \cup R_{-}(\lambda)\right)$.

Now, we consider case (iii) of Corollary 6.4. Namely, we show that if we rescale and reparameterize $\gamma$ and project off its $\tau$ component, we obtain a generalized broken geodesic (of $h$ ) in $\partial X$, broken at $\mathcal{C}$. This is a notion completely analogous to that of our generalized broken bicharacteristics, and we proceed to define it. Again, we need to introduce a 'compressed' cotangent bundle. The metric $h$ on $\partial X$ naturally identifies the cotangent bundle $T^{*} C$ of $C \in \mathcal{C}$ as a subset of $T^{*} \partial X$. The compressed cotangent bundle of $\partial X$ is then

$$
\dot{T}^{*} \partial X=\bigcup_{a} T_{C_{a}^{\prime}}^{*} C_{a}
$$

It is topologized by the projection $\pi_{\partial}: T^{*} \partial X \rightarrow \dot{T}^{*} \partial X$. We also define the compressed cosphere bundle as the image of $S^{*} \partial X$ under $\pi_{\partial}$; here $S^{*} \partial X$ is the set of covcectors of unit length:

$$
\dot{S}^{*} \partial X=\pi_{\partial}\left(S^{*} \partial X\right) .
$$

The restriction of $\pi_{\partial}$ to $\dot{S}^{*} \partial X$ is denoted by $\hat{\pi}_{\partial}$. This plays a role analogous to that of $\dot{\Sigma}$. We also define its tangential and normal parts:

$$
\dot{S}_{n}^{*} \partial X=\bigcup_{a}\left\{\zeta \in T_{C_{a}^{\prime}}^{*} C_{a} \cap \dot{S}^{*} \partial X: \hat{\pi}_{\partial}^{-1}(\zeta) \text { consists of more than one point }\right\}
$$

and

$$
\dot{S}_{t}^{*} \partial X=\bigcup_{a}\left\{\zeta \in T_{C_{a}^{\prime}}^{*} C_{a} \cap \dot{S}^{*} \partial X: \hat{\pi}_{\partial}^{-1}(\zeta) \text { consists of exactly one point }\right\} .
$$

Generalized broken geodesics are then defined as follows.

DEFINITION 6.6. - A generalized broken geodesic of $h$ is a continuous map $\gamma_{\partial}: I \rightarrow \dot{S}^{*} \partial X$, where $I \subset \mathbb{R}$ is an interval, satisfying the following requirements:

(i) If $\zeta_{0}=\gamma_{\partial}\left(t_{0}\right) \in \dot{S}_{t}^{*} \partial X$ then for all $\pi_{\partial}$-invariant functions $f \in \mathcal{C}^{\infty}\left(T^{*} \partial X\right)$,

$$
\frac{d}{d t}\left(f_{\pi_{\partial}} \circ \gamma_{\partial}\right)\left(t_{0}\right)=H_{\frac{1}{2} h} f\left(\tilde{\zeta}_{0}\right), \quad \tilde{\zeta}_{0}=\hat{\pi}_{\partial}^{-1}\left(\zeta_{0}\right)
$$

(ii) If $\zeta_{0}=\gamma_{\partial}\left(t_{0}\right) \in \dot{S}_{n}^{*} \partial X \cap T_{C_{a}^{\prime}}^{*} C_{a}$ then there exists $\varepsilon>0$ such that

$$
t \in I, \quad 0<\left|t-t_{0}\right|<\varepsilon \Rightarrow \gamma_{\partial}(t) \notin T_{C_{a}^{\prime}}^{*} C_{a} .
$$


Remark 6.7. - Sometimes, with an abuse of terminology, we also say that the projection of a generalized broken geodesic to $\partial X$ (via the projection $\dot{S}^{*} \partial X \rightarrow \partial X$ inherited from $T \partial X$ ) is a generalized broken geodesic. Indeed, this was the terminology used in the introduction.

The metric $g$ gives rise to a product decomposition

$$
{ }^{\mathrm{sc}} T_{\partial X}^{*} X=\mathbb{R}_{\tau} \times T^{*} \partial X
$$

The compressed scattering cotangent bundle is thus also naturally a product:

$$
{ }^{\mathrm{sc}} \dot{T}^{*} X=\mathbb{R}_{\tau} \times \dot{T}^{*} \partial X .
$$

We sometimes write the product variables as $\xi=\left(\tau, \xi^{\prime \prime}\right)$. We write

$$
p:{ }^{\mathrm{sc}} \dot{T}^{*} X \rightarrow \dot{T}^{*} \partial X
$$

for the projection to the second factor. Note that ${ }^{\mathrm{sc}} \dot{T}^{*} X$ inherits a natural $\mathbb{R}$-action from ${ }^{\mathrm{sc}} T_{\partial X}^{*} X$, and if $\xi \in \dot{\Sigma}, \tau(\xi)^{2} \neq \lambda$, then $\zeta=p\left(\left(\lambda-\tau(\xi)^{2}\right)^{-1 / 2} \xi\right) \in \dot{S}^{*} \partial X$ since $h=\lambda-\tau^{2}$ on $\dot{\Sigma}$.

We also reparameterize generalized broken bicharacteristics $\gamma$ satisfying (iii) of Corollary 6.4 by letting $s=S(t)$ where $S$ satisfies $d S / d t=2\left(\lambda-\tau(\gamma(t))^{2}\right)^{1 / 2}$, with $S\left(t_{0}\right)=s_{0}$ picked arbitrarily. We have the following result.

Proposition 6.8. - Suppose that $\gamma: I \rightarrow \dot{\Sigma}$ is a generalized broken bicharacteristic which is disjoint from $R_{+}(\lambda) \cup R_{-}(\lambda)$. Then $\gamma \circ S^{-1}: J \rightarrow \dot{\Sigma}, S$ defined above, is given by

$$
\tau=\sqrt{\lambda} \cos \left(s-s_{1}\right), \quad \xi^{\prime \prime}=\sqrt{\lambda} \sin \left(s-s_{1}\right) \gamma_{\partial}(s)
$$

where $s_{1}$ is an appropriate constant and $\gamma_{\partial}: J \rightarrow \dot{S}^{*} \partial X$ is a generalized broken geodesic, broken at $\mathcal{C}$. If $I=\mathbb{R}$, then $J=\left(s_{1}, s_{1}+\pi\right)$, in particular $J$ has length $\pi$, and correspondingly the projection of $\gamma_{\partial}$ to $\partial X$ is a curve of length $\pi$.

Proof. - Let

$$
\gamma_{\partial}(s)=p\left(\frac{\gamma\left(S^{-1}(s)\right)}{\sqrt{\lambda-\tau\left(\gamma\left(S^{-1}(s)\right)\right)^{2}}}\right) .
$$

Condition (ii) of Definition 6.2 implies (ii) of Definition 6.6 immediately. Let $f \in \mathcal{C}^{\infty}\left(T^{*} \partial X\right)$ be a $\pi_{\partial}$-invariant function. Let

$$
F(\xi)=f\left(p\left(\left(\lambda-\tau(\xi)^{2}\right)^{-1 / 2} \xi\right)\right)
$$

here we slightly abuse the notation and write $p:{ }^{\mathrm{sc}} T_{\partial X}^{*} X \rightarrow T^{*} \partial X$. Then $F$ is $\pi$-invariant, so (i) of Definition 6.2 applies and gives $d\left(F_{\pi} \circ \gamma\right) / d t\left(t_{0}\right)$. Since $\left(F_{\pi} \circ \gamma\right) \circ S^{-1}=f_{\pi_{\partial}} \circ \gamma_{\partial}$, the chain rule and a short calculation of ${ }^{\mathrm{sc}} H_{g} F$ gives (i) of Definition 6.6. The first equation in (6.34) follows since along $\gamma, d s / d \tau=(d s / d t)(d \tau / d t)^{-1}=-\left(\lambda-\tau^{2}\right)^{-1 / 2}$. As $\left(\lambda-\tau^{2}\right)^{1 / 2}=$ $\sqrt{\lambda} \sin \left(s-s_{1}\right)$, the second equation follows as well. Since $\tau \rightarrow \mp \sqrt{\lambda}$ along $\gamma$ as $t \rightarrow \pm \infty$ and $\tau$ is decreasing, we deduce the last statement.

It is useful to introduce a relation on $\dot{S}^{*} \times \dot{\Sigma}(\lambda)$ using the structure of the generalized broken bicharacteristics given in this proposition.

Definition 6.9. - Suppose $\xi \in \dot{\Sigma}(\lambda) \backslash\left(R_{-}(\lambda) \cup R_{+}(\lambda)\right), \zeta \in \dot{S}^{*} \partial X$. We say that $\xi \sim_{-} \zeta$ if there is a generalized broken bicharacteristic $\gamma: \mathbb{R} \rightarrow \dot{\Sigma}(\lambda)$ with $\gamma\left(t_{0}\right)=\xi$ such that 
$\gamma_{\partial}:(a, a+\pi) \rightarrow S^{*} \partial X$, as in the above Proposition, satisfies $\lim _{s \rightarrow a+} \gamma_{\partial}(s)=\zeta$. We define $\xi \sim_{+} \zeta$ similarly by replacing $a+$ in the limit by $(a+\pi)-$.

We also need to analyze the uniform behavior of generalized broken bicharacteristics. Here we quote Lebeau's results; they can also be proved completely analogously to the proof of Proposition 6.3 given here in the Appendix.

Proposition 6.10 (Lebeau, [18, Proposition 5]). - Suppose that $K$ is a compact subset of $\dot{\Sigma}$, $\gamma_{n}:[a, b] \rightarrow K$ is a sequence of generalized broken bicharacteristics which converge uniformly to $\gamma$. Then $\gamma$ is a generalized broken bicharacteristic.

Proposition 6.11 (Lebeau, [18, Proposition 6]). - Suppose that $K$ is a compact subset of $\dot{\Sigma},[a, b] \subset \mathbb{R}$ and

$$
\mathcal{R}=\{\text { generalized broken bicharacteristics } \gamma:[a, b] \rightarrow K\} .
$$

If $\mathcal{R}$ is not empty then it is compact in the topology of uniform convergence.

COROLlary 6.12 (Lebeau, [18, Corollaire 7]). - If $\gamma:(a, b) \rightarrow \mathbb{R}$ is a generalized broken bicharacteristic then $\gamma$ extends to $[a, b]$.

\section{Generalized broken bicharacteristics for totally geodesic $\mathcal{C}$}

We next examine the generalized broken bicharacteristics if all elements of $\mathcal{C}$ are totally geodesic with respect to $h$. First we prove that generalized broken bicharacteristics $\gamma: I \rightarrow \dot{\Sigma}$ with $\gamma\left(t_{0}\right)=\xi_{0}, \xi_{0} \in \Sigma_{t}(\lambda) \cap{ }^{\mathrm{sc}} T_{C_{a}^{\prime}}^{*}\left(C_{a} ; X\right)$ are actually bicharacteristics of ${ }^{\mathrm{sc}} H_{g}$ (and hence stay in $\left.{ }^{\mathrm{sc}} T_{C_{a}^{\prime}}^{*}\left(C_{a} ; X\right)\right)$ for $t$ near $t_{0}$.

Proposition 7.1. - Suppose that all elements of $\mathcal{C}$ are totally geodesic with respect to $h$. Let $\gamma: I \rightarrow \dot{\Sigma}$ be a generalized broken bicharacteristic,

$$
\gamma\left(t_{0}\right)=\xi_{0} \in\left(\Sigma_{t}(\lambda) \cap{ }^{\mathrm{sc}} T_{C_{a}^{\prime}}^{*}\left(C_{a} ; X\right)\right) \backslash\left(R_{+}(\lambda) \cup R_{-}(\lambda)\right) .
$$

Then for $t \in J, J$ a neighborhood of $t_{0}$, we have $\gamma(t) \in \Sigma_{t}(\lambda) \cap{ }^{\mathrm{sc}} T_{C_{a}^{\prime}}^{*}\left(C_{a} ; X\right)$, and $\left.\gamma\right|_{J}$ is a bicharacteristic of ${ }^{\mathrm{sc}} H_{g}$.

Proof. - Our strategy consists of constructing a $\pi$-invariant function $\phi$ with ${ }^{\mathrm{sc}} H_{g} \phi \geqslant c>0$ in a neighborhood of $\hat{\pi}^{-1}\left(\xi_{0}\right)$. Thus, by Proposition 6.3, $d /\left.d t\left(\phi_{\pi}(\gamma)\right)\right|_{t \pm} \geqslant c>0$ for $t \in J, J$ sufficiently small, so $\phi_{\pi} \circ \gamma$ is increasing there. This will allow us to draw the desired conclusion for the correct choice of $\phi$. We remark that this $\phi$ will reappear in the proof of the propagation estimate in Proposition 10.6. Moreover, it is essentially the same as the corresponding function in the three-body propagation estimate [40, Proposition 15.4], though we will use slightly different methods to estimate ${ }^{\mathrm{sc}} H_{g} \phi$.

In fact, first we find a $\pi$-invariant function $\omega$ such that ${ }^{\mathrm{sc}} H_{g}$ will be appropriately small near $\hat{\pi}^{-1}\left(\xi_{0}\right)$. So introduce coordinates centered at $C_{a}^{\prime}$ as after Definition 6.2. Then the metric function takes the form

$$
h=\sum h_{n n}^{i j}(y, z) \mu_{i} \mu_{j}+2 \sum h_{n t}^{i j}(y, z) \mu_{i} \nu_{j}+\sum h_{t t}^{i j}(y, z) \nu_{i} \nu_{j}
$$

with

$$
h_{n n}^{i j}(0,0)=\delta^{i j}, \quad h_{n t}^{i j}(0, z)=0,
$$


and, due to the assumption that $C_{a}$ is totally geodesic,

$$
\partial_{y} h_{t t}^{i j}(0, z)=0
$$

We write

$$
\tilde{h}(z, \nu)=\sum h_{t t}^{i j}(0, z) \nu_{i} \nu_{j}
$$

for the restriction of the tangential part of the metric function to $C_{a}$, so

$$
\left.h\right|_{y=0}=\tilde{h}+\sum h_{n n}^{i j}(0, z) \mu_{i} \mu_{j}
$$

Now, the Hamilton vector field of $h$ is given by

$$
\begin{aligned}
H_{h}= & 2 \sum_{i, j} h_{n n}^{i j} \mu_{j} \partial_{y_{i}}+2 \sum_{i, j} h_{n t}^{i j} \mu_{i} \partial_{z_{j}}+2 \sum_{i j} h_{n t}^{i j} \nu_{j} \partial_{y_{i}}+2 \sum_{i, j} h_{t t}^{i j} \nu_{j} \partial_{z_{i}} \\
& +\sum_{i, j, k}\left(\partial_{z_{k}} h_{n n}^{i j}\right) \mu_{i} \mu_{j} \partial_{\nu_{k}}+2 \sum_{i, j, k}\left(\partial_{z_{k}} h_{n t}^{i j}\right) \mu_{i} \nu_{j} \partial_{\nu_{k}}+\sum_{i, j, k}\left(\partial_{z_{k}} h_{t t}^{i j}\right) \nu_{i} \nu_{j} \partial_{\nu_{k}}+W^{\prime}
\end{aligned}
$$

with $W^{\prime}=\sum \alpha_{j} \partial_{\mu_{j}}$. Hence, if $\omega \in \mathcal{C}^{\infty}\left(\mathbb{R}_{z}^{m-1} \times \mathbb{R}_{\tau, \nu}^{m}\right)$ then

$$
\left.H_{h} \omega\right|_{y=0}=H_{\tilde{h}} \omega+\sum_{k}\left(\partial_{z_{k}}(h-\tilde{h})\right) \partial_{\nu_{k}} \omega
$$

Now, $\mu$, hence $h-\tilde{h}$, is small near $\hat{\pi}^{-1}\left(\xi_{0}\right)$, so to model

$$
{ }^{\mathrm{sc}} H_{g}=2 \tau\left(\mu \cdot \partial_{\mu}+\nu \cdot \partial_{\nu}\right)-2 h \partial_{\tau}+H_{h}
$$

we introduce the vector field

$$
W=2 \tau\left(\nu \cdot \partial_{\nu}\right)-2 \tilde{h} \partial_{\tau}+H_{\tilde{h}}
$$

locally (near $\xi_{0}$ ) on ${ }^{\mathrm{sc}} T^{*}\left(C_{a} ; X\right)$. Thus, we have

$$
\left.{ }^{\mathrm{sc}} H_{g} \omega\right|_{y=0}=W \omega-2(h-\tilde{h}) \partial_{\tau} \omega+\sum_{k}\left(\partial_{z_{k}}(h-\tilde{h})\right) \partial_{\nu_{k}} \omega
$$

which is small if $W \omega$ is small.

We define $\omega$ as follows. First, $W \tau=-2 \tilde{h}$, and $\tilde{h}_{z_{0}}\left(\nu_{0}\right) \neq 0$ since $\xi_{0} \notin R_{+}(\lambda) \cup R_{-}(\lambda)$, so near $\xi_{0}, W \tau \neq 0$, i.e. $W$ is transversal to the hypersurface $\tau=\tau_{0}$. Thus, near $\xi_{0}$ in ${ }^{\text {sc }} T^{*}\left(C_{a} ; X\right)$ we can solve the Cauchy problem

$$
W \omega=0,\left.\quad \omega\right|_{\tau=\tau_{0}}=\left(z-z_{0}\right)^{2}+\left(\nu-\nu_{0}\right)^{2} .
$$

Since $\omega$ and $d \omega$ vanish at $\xi_{0}$, the same holds on the bicharacteristic of $W$ through $\xi_{0}$, but $\omega \geqslant 0$ and the Hessian is still positive in directions transversal to the bicharacteristics as these hold at $\xi_{0}$. Moreover, by [12, Lemma 7.7.2],

$$
|d \omega| \leqslant C \omega^{1 / 2}
$$

4 SÉRIE - TOME $34-2001-\mathrm{N}^{\circ} 3$ 
Let

$$
r_{0}=\tau^{2}+\tilde{h}_{z}(\nu)-\lambda,
$$

so $W r_{0}=0$. At $\tau=\tau_{0}$ we have $r_{0}=\tilde{h}_{z}(\nu)-\tilde{h}_{z_{0}}\left(\nu_{0}\right)$, so

$$
\left|r_{0}\right| \leqslant C^{\prime}|d \omega| \leqslant C^{\prime \prime} \omega^{1 / 2}
$$

when $\tau=\tau_{0}$, and then $W \omega=0=W r_{0}$ implies that this inequality holds everywhere. Therefore,

$$
|\tilde{h}-h| \leqslant\left|\lambda-\tau^{2}-h\right|+\left|\lambda-\tau^{2}-\tilde{h}\right| \leqslant\left|\lambda-\tau^{2}-h\right|+C \omega^{1 / 2} .
$$

Now,

$$
\begin{aligned}
{ }^{\mathrm{sc}} H_{g} \omega={ }^{\mathrm{sc}} H_{g} \omega-W \omega= & -2(h-\tilde{h}) \partial_{\tau} \omega \\
& +2 \sum_{i, j} h_{n t}^{i j}(y, z) \mu_{i} \partial_{z_{j}} \omega+2 \sum_{i, j}\left(h_{t t}^{i j}(y, z)-h_{t t}^{i j}(0, z)\right) \nu_{j} \partial_{z_{i}} \omega \\
& +\sum_{i, j, k} \partial_{z_{k}} h_{n n}^{i j}(y, z) \mu_{i} \mu_{j} \partial_{\nu_{k}} \omega+2 \sum_{i, j, k} \partial_{z_{k}} h_{n t}^{i j}(y, z) \mu_{i} \nu_{j} \partial_{\nu_{k}} \omega \\
& +\sum_{i, j, k} \partial_{z_{k}}\left(h_{t t}^{i j}(y, z)-h_{t t}^{i j}(0, z)\right) \nu_{i} \nu_{j} \partial_{\nu_{k}} \omega .
\end{aligned}
$$

Thus, using (7.3)-(7.4), for some $C, C^{\prime}>0$ we have

$$
\begin{aligned}
\left|{ }^{\mathrm{sc}} H_{g} \omega-W \omega\right| & \leqslant C^{\prime}\left(\left|\tau^{2}+h-\lambda\right|+\omega^{1 / 2}+|y|^{2}+|\mu|^{2}+|\mu||y|\right)|d \omega| \\
& \leqslant C\left(\left|\tau^{2}+h-\lambda\right|+\omega^{1 / 2}+|y|^{2}+|\mu|^{2}\right) \omega^{1 / 2} .
\end{aligned}
$$

Next, note that

$$
{ }^{\mathrm{sc}} H_{g}|y|^{2}=4 \sum_{i, j} h_{n n}^{i j} \mu_{j} y_{i}+4 \sum_{i, j} h_{n t}^{i j} \nu_{j} y_{i}
$$

so by (7.3),

$$
\left.\left|{ }^{\mathrm{sc}} H_{g}\right| y\right|^{2}|\leqslant C| y \mid(|y|+|\mu|)
$$

For $\varepsilon>0$ let

$$
\phi^{(\varepsilon)}=\phi=\tau_{0}-\tau+\varepsilon^{-1}|y|^{2}+\varepsilon^{-2} \omega .
$$

Thus,

(7.22) $\left|{ }^{\mathrm{sc}} H_{g} \phi-2 h\right| \leqslant C\left(\varepsilon^{-1}|y|(|y|+|\mu|)+\varepsilon^{-2} \omega^{1 / 2}\left(|y|^{2}+|\mu|^{2}+\left|\tau^{2}+h-\lambda\right|+\omega^{1 / 2}\right)\right)$.

We next estimate $\mu$. First, as $h_{n n}^{i j}(0,0)=\delta_{i j}, h_{n n}$ is positive definite in a small neighborhood of $(0,0)$ and

$$
|\mu|^{2} \leqslant 2 \sum_{i, j} h_{n n}^{i j}(y, z) \mu_{i} \mu_{j}
$$

there. On the other hand,

$$
\sum_{i, j} h_{n n}^{i j}(y, z) \mu_{i} \mu_{j}=h-\tilde{h}-\sum_{i, j} h_{n t}^{i j}(y, z) \mu_{i} \nu_{j}-\sum_{i, j}\left(h_{t t}^{i j}(y, z)-h_{t t}^{i j}(0, z)\right) \nu_{i} \nu_{j}
$$


so

$$
\left|\sum_{i, j} h_{n n}^{i j}(y, z) \mu_{i} \mu_{j}\right| \leqslant|h-\tilde{h}|+C_{1}|y||\mu|+C_{2}|y|^{2} .
$$

Moving $C_{1}|y||\mu|$ to the right hand side and completing the square gives

$$
\left(|\mu|-C_{3}|y|\right)^{2} \leqslant|h-\tilde{h}|+C_{4}|y|^{2},
$$

so

$$
|\mu| \leqslant C\left(|h-\tilde{h}|^{1 / 2}+|y|\right), \quad \text { i.e. }|\mu|^{2} \leqslant C^{\prime}\left(|h-\tilde{h}|+|y|^{2}\right) .
$$

We can finally estimate ${ }^{\mathrm{sc}} H_{g} \phi$, using (7.16) as well:

$$
\begin{aligned}
\left|{ }^{\mathrm{sc}} H_{g} \phi-2 h\right| \leqslant & C\left(\varepsilon^{-1}|y|\left(|y|+\omega^{1 / 4}+\left|\tau^{2}+h-\lambda\right|^{1 / 2}\right)\right. \\
& \left.+\varepsilon^{-2} \omega^{1 / 2}\left(|y|^{2}+\left|\tau^{2}+h-\lambda\right|+\omega^{1 / 2}\right)\right) .
\end{aligned}
$$

Note that $\phi_{\pi}\left(\xi_{0}\right)=0$, so near $\pi^{-1}\left(\xi_{0}\right), \phi$ is small. So now suppose that $0<\delta<1$ and

$$
\phi \leqslant 2 \delta \quad \text { and } \quad \tau-\tau_{0} \leqslant 2 \delta .
$$

Then

$$
\varepsilon^{-1}|y|^{2}+\varepsilon^{-2} \omega \leqslant 4 \delta,
$$

so $|y| \leqslant(4 \varepsilon \delta)^{1 / 2}, \omega \leqslant 4 \varepsilon^{2} \delta$. Hence, under the additional assumption

$$
\left|\tau^{2}+h-\lambda\right|<\varepsilon \delta
$$

i.e. that $\tilde{\xi}=(y, z, \tau, \mu, \nu)$ sufficiently close to $\Sigma_{\Delta-\lambda}$, we have

$$
\left|{ }^{\mathrm{sc}} H_{g} \phi-2 h\right| \leqslant C\left(\varepsilon^{-1}(\varepsilon \delta)^{1 / 2}\left(\varepsilon^{2} \delta\right)^{1 / 4}+\varepsilon^{-2} \varepsilon^{2} \delta\right) \leqslant C^{\prime} \delta^{3 / 4} .
$$

Since $h\left(\hat{\pi}^{-1}\left(\xi_{0}\right)\right)>0$, we have $h(\tilde{\xi}) \geqslant 2 c>0$ in a neighborhood of $\hat{\pi}^{-1}\left(\xi_{0}\right)$. Now choose $\delta_{0}>0$ sufficiently small, so that $C^{\prime} \delta_{0}^{3 / 4}<c$. Note that this requirement is independent of $\varepsilon$. We thus conclude that for $\delta \in\left(0, \delta_{0}\right), \tilde{\xi}$ satisfying (7.29) and (7.31), we have

$$
{ }^{\mathrm{sc}} H_{g} \phi(\xi) \geqslant c>0 \text {. }
$$

Now, using the result of Proposition 6.3, let $\tilde{\xi}_{ \pm}(t) \in \Sigma(\Delta-\lambda)$ be the unique points such that $\pi\left(\tilde{\xi}_{ \pm}(t)\right)=\gamma(t)$ and for all $\pi$-invariant $f$

$$
\left.\left(\frac{d}{d t}\right)\left(f_{\pi} \circ \gamma\right)\right|_{t \pm}={ }^{\mathrm{sc}} H_{g} f\left(\tilde{\xi}_{ \pm}(t)\right) .
$$

Choosing a sufficiently small open interval $J$ around $t_{0}, \tau(\gamma(t))$, hence $\tau\left(\tilde{\xi}_{ \pm}(t)\right)$, automatically satisfies (7.29) for $t \in J$, while (7.31) holds automatically as $\tilde{\xi}_{ \pm}(t) \in \Sigma(\Delta-\lambda)$. Thus, applying (7.34) with $\phi$ in place of $f$, we see that, with

$$
g(t)=\phi_{\pi} \circ \gamma(t)
$$

$4^{\mathrm{e}}$ SÉRIE - TOME $34-2001-\mathrm{N}^{\circ} 3$ 
we have

$$
t \in J \quad \text { and } \quad g(t) \leqslant\left. 2 \delta \Rightarrow\left(\frac{d g}{d t}\right)\right|_{t \pm} \geqslant c>0 .
$$

As $g$ is continuous and $g\left(t_{0}\right)=0$, this shows that $g$ is increasing on $J \cap\left(-\infty, t_{0}\right]$. To see this, first note that $g(t)<2 \delta$ on $J \cap\left(-\infty, t_{0}\right]$, for otherwise $g^{-1}(\{2 \delta\}) \cap\left(-\infty, t_{0}\right] \cap J$ is not empty, $g^{-1}(\{2 \delta\}) \cap\left(-\infty, t_{0}\right]$ is closed, so taking $t_{1}=\sup \left(g^{-1}(\{2 \delta\}) \cap\left(-\infty, t_{0}\right]\right)<t_{0}$ and $t_{1} \in J$. Thus, for $t \in\left[t_{1}, t_{0}\right], g$ is differentiable from either side at $t$ and the derivatives are both positive, so $g$ is increasing on $\left[t_{1}, t_{0}\right]$, hence $g\left(t_{1}\right) \leqslant g\left(t_{0}\right)=0$ contradicting $g\left(t_{1}\right)=2 \delta$. Thus, $g<2 \delta$ on $J \cap(-\infty, 0]$, so $g$ is increasing here, so $g(t) \leqslant 0$ for $t \in J \cap\left(-\infty, t_{0}\right)$. Taking into account the definition of $\phi$ we immediately deduce that

$$
|y(\gamma(t))| \leqslant C \varepsilon^{1 / 2}, \quad t \in J \cap\left(-\infty, t_{0}\right) .
$$

Since $\varepsilon \in(0,1)$ is arbitrary, we conclude that $y(\gamma(t))=0$ for $t \in J \cap\left(-\infty, t_{0}\right]$, so $\gamma(t) \in$ ${ }^{\mathrm{sc}} T^{*}\left(C_{a} ; X\right)$ for such $t$. Similarly, $\omega(\gamma(t))=0$ for such $t$, so by the construction of $\omega, \gamma(t)$ is the integral curve of $W$ through $\xi_{0}$ (for $t \in J, t \leqslant t_{0}$ ). Of course, a similar argument (with a change of sign in $\tau_{0}-\tau$ in (7.21)) works for $J \cap[0, \infty)$, so we conclude that $\left.\gamma\right|_{J} \subset{ }^{\mathrm{sc}} T_{C_{a}^{\prime}}^{*}\left(C_{a} ; X\right)$ and $\left.\gamma\right|_{J}$ is an integral curve of $W$. As $W$ preserves $\tau^{2}+\tilde{h}$ (being essentially its rescaled Hamilton vector field), $\tau^{2}(\gamma(t))+\tilde{h}(\gamma(t))=\lambda, t \in J$, so $\left.\gamma\right|_{J} \subset \Sigma_{t}(\lambda)$, and hence at $\hat{\pi}^{-1}\left(\left.\gamma\right|_{J}\right)$, ${ }^{\text {sc }} H_{g}$ and $W$ agree and $\left.\gamma\right|_{J}$ is a bicharacteristic of ${ }^{\mathrm{sc}} H_{g}$ as claimed.

Next, we prove that if $\xi_{0} \in \Sigma_{n}(\lambda) \cap{ }^{\mathrm{sc}} T_{C_{a}^{\prime}}^{*}\left(C_{a} ; X\right), \gamma\left(t_{0}\right)=\xi_{0}, \gamma$ is a generalized broken bicharacteristic, then for a sufficiently small $\delta>0,\left.\gamma\right|_{[0, \delta]}$ is a generalized broken bicharacteristic of $\Delta-\lambda$, broken at $\mathcal{C}^{\prime} \subset \mathcal{C}$, where $\mathcal{C}^{\prime}$ is cleanly intersecting and $C_{a} \notin \mathcal{C}^{\prime}$. This will not use that $\mathcal{C}$ is totally geodesic.

Proposition 7.2. - Suppose that $\xi_{0} \in \Sigma_{n}(\lambda) \cap{ }^{\mathrm{sc}} T_{C_{a}^{\prime}}^{*}\left(C_{a} ; X\right), \gamma$ is a generalized broken geodesic with $\gamma\left(t_{0}\right)=\xi_{0}$ and $\tilde{\xi}_{+}$is as in Proposition 6.3. Suppose that $\left.\tilde{\xi}_{+} \in{ }^{\mathrm{sc}} T^{*}\left(C_{b} ; X\right)\right)$ and $b$ is minimal with this property (i.e. $C_{c} \subset C_{b}$ and $\tilde{\xi}_{+} \in{ }^{\mathrm{sc}} T^{*}\left(C_{c} ; X\right)$ imply $c=b$ ). Let

$$
\mathcal{C}^{\prime}=\mathcal{C} \backslash\left\{C_{c}: C_{c} \cap C_{b} \subset C_{a}\right\} .
$$

Then for sufficiently small $\delta>0,\left.\gamma\right|_{[0, \delta]}$ is a generalized broken bicharacteristic of $\Delta-\lambda$, broken at $\mathcal{C}^{\prime}$, and $\gamma((0, \delta])$ is disjoint from ${ }^{\mathrm{sc}} T^{*}\left(C_{c} ; X\right)$ if $C_{c} \notin \mathcal{C}^{\prime}$.

Proof. - Let $b$ be as above and introduce local coordinates centered at $C_{a}^{\prime}$. We may assume that $C_{b}$ is given by $y^{\prime}=0$ for a suitable splitting $y=\left(y^{\prime}, y^{\prime \prime}\right)$. Thus, $\xi_{+}$is of the form $\tilde{\xi}_{+}=\left(0,0, \tau_{0}, 0, \mu_{0}^{\prime \prime}, \nu_{0}\right)$, and as $\tilde{\xi}_{+} \in \Sigma_{n}(\lambda), \mu_{0}^{\prime \prime} \neq 0$. By Proposition 6.3, taking into account that $y$ is $\pi$-invariant,

$$
d\left(y_{j}^{\prime} \circ \gamma\right) /\left.d t\right|_{t_{0}+}=0, \quad d\left(y_{j}^{\prime \prime} \circ \gamma\right) /\left.d t\right|_{t_{0}+}=\left(\mu_{0}^{\prime \prime}\right)_{j} .
$$

Since $\mu_{0}^{\prime \prime} \neq 0$, there exist $c>0, \delta_{0}>0$, such that $\left|y^{\prime \prime}(\gamma(t))\right| \geqslant c\left(t-t_{0}\right)$ for $t \in\left(t_{0}, t_{0}+\delta_{0}\right)$, while for any $\varepsilon>0$ there exists $\delta_{1}>0$ such that $\left|y^{\prime}(\gamma(t))\right| \leqslant \varepsilon\left(t-t_{0}\right)$ for $t \in\left(t_{0}, t_{0}+\delta_{1}\right)$. In particular, for any $\varepsilon>0$ there exists $\delta>0$ such that for $t \in\left(t_{0}, t_{0}+\delta\right)$ we have $\left|y^{\prime}(\gamma(t))\right| /\left|y^{\prime \prime}(\gamma(t))\right| \leqslant \varepsilon$. By choosing $\varepsilon>0$ sufficiently small we can thus make sure that $\gamma(t) \notin C_{c}$ for $t \in\left(t_{0}, t_{0}+\delta\right]$ if $C_{c} \notin \mathcal{C}^{\prime}$. Hence, $\left.\gamma\right|_{\left[t_{0}, t_{0}+\delta\right]}$ can be regarded as a curve in $\bigcup_{C_{c} \in \mathcal{C}^{\prime}}{ }^{\mathrm{sc}} T_{C_{c}^{\prime}}^{*}\left(C_{c} ; X\right), C_{c}^{\prime}$ taken with respect to $\mathcal{C}^{\prime}$, if we let $\gamma\left(t_{0}\right)=\pi_{0 b}\left(\tilde{\xi}_{0}\right) \in{ }^{\mathrm{sc}} T^{*}\left(C_{b} ; X\right)$. Of course, $\left.\gamma\right|_{\left(t_{0}, t_{0}+\delta\right]}$ is a generalized broken bicharacteristic, broken at $\mathcal{C}^{\prime}$ (since it has no points above $\mathcal{C} \backslash \mathcal{C}^{\prime}$ ). Thus, by Corollary 6.12, 
$\left.\gamma\right|_{\left(t_{0}, t_{0}+\delta\right]}$ extends to a generalized broken bicharacteristic, broken at $\mathcal{C}^{\prime}$, defined on $\left[t_{0}, t_{0}+\delta\right]$; by continuity of $\gamma$ this must coincide with $\gamma$, so $\gamma$ is a generalized broken bicharacteristic, broken at $\mathcal{C}^{\prime}$, as claimed.

We can combine the previous results to deduce the structure of the generalized broken bicharacteristics if $\mathcal{C}$ is totally geodesic.

Proposition 7.3. - Suppose that $\mathcal{C}$ is totally geodesic with respect to $h$ and $\gamma$ is a generalized broken bicharacteristic, broken at $\mathcal{C}$ with $\xi_{0}=\gamma\left(t_{0}\right) \in{ }^{\mathrm{sc}} T_{C_{a}^{\prime}}^{*}\left(C_{a} ; X\right)$. Then there exists $\delta>0$ such that both $\left.\gamma\right|_{\left[t_{0}, t_{0}+\delta\right)}$ and $\left.\gamma\right|_{\left(t_{0}-\delta, t_{0}\right]}$ are bicharacteristics of ${ }^{\mathrm{sc}} H_{g}$.

Proof. - If $\xi_{0} \in R_{+}(\lambda) \cup R_{-}(\lambda)$ then $\gamma(t)=\xi_{0}$ for $t$ near $t_{0}$ by Proposition 6.5, hence near $t_{0}, \gamma$ is a ( $\pi$-projected) bicharacteristic of ${ }^{\mathrm{sc}} H_{g}$ (as ${ }^{\mathrm{sc}} H_{g}$ vanishes at $R_{+}(\lambda) \cup R_{-}(\lambda)$ ). If $\xi_{0} \in \Sigma_{t}(\lambda) \backslash\left(R_{+}(\lambda) \cup R_{-}(\lambda)\right)$ then Proposition 7.1 applies and proves the result. If $\xi_{0} \in \Sigma_{n}(\lambda)$, then with $\mathcal{C}^{\prime}$ as in Proposition 7.2, $\left.\gamma\right|_{[0, \delta)}$ is a generalized broken bicharacteristic, broken at $\mathcal{C}^{\prime}$, with $\gamma\left(t_{0}\right) \in{ }^{\mathrm{sc}} T_{C_{b}^{\prime}}^{*}\left(C_{b} ; X\right) \cap \Sigma_{t}(\lambda)$ (prime taken with respect to $\mathcal{C}^{\prime}$ ). Thus, Proposition 7.1 applies again and proves the result.

A compactness argument gives at once

COROLLARY 7.4. - If $\gamma:[a, b] \rightarrow \dot{\Sigma}$ is a generalized broken bicharacteristic, broken at $\mathcal{C}$, and $\mathcal{C}$ is totally geodesic, then there exist $t_{0}=a<t_{1}<t_{2}<\cdots<t_{m}=b$ such that $\left.\left.\gamma\right|_{[} t_{j}, t_{j+1}\right]$ is a bicharacteristic of $\Delta-\lambda$ (i.e. it is not broken).

\section{Positive operators}

In the following two sections we discuss technical points of the microlocal positive commutators constructions. In this section we show roughly speaking that the positivity of the indicial operators of $A \in \Psi_{\mathrm{Sc}}^{-\infty, 0}(X, \mathcal{C})$ implies the positivity of $A$ modulo compact operators. We prove this by constructing an approximate square root of $A$. In the next section we examine commutators $[A, H]$ in more detail.

Throughout this section we assume that $H$ is a many-body Hamiltonian. We start with the basic square root construction.

LEMma 8.1. - Suppose that $H$ is a many-body Hamiltonian and $\lambda \in \mathbb{R}$. Suppose also that $A \in \Psi_{\mathrm{Sc}}^{-\infty, 0}(X, \mathcal{C})$ is self-adjoint, and for some $c>0$ and $\psi \in \mathcal{C}_{c}^{\infty}(\mathbb{R})$ which is identically 1 near $\lambda$,

$$
\psi(H) A \psi(H) \geqslant c \psi(H)^{2} .
$$

Then for any $c^{\prime} \in(0, c)$ and $\phi \in \mathcal{C}_{c}^{\infty}(\mathbb{R})$ such that

$$
\operatorname{supp} \phi \cap \operatorname{supp}(1-\psi)=\emptyset,
$$

there exists $B \in \Psi_{\mathrm{Sc}}^{-\infty, 0}(X, \mathcal{C})$ such that

$$
\phi(H)\left(A-c^{\prime}\right) \phi(H)=\phi(H) B^{*} B \phi(H) .
$$

Proof. - Let

$$
P=\psi(H) A \psi(H)+c\left(\mathrm{Id}-\psi(H)^{2}\right) \in \Psi_{\mathrm{Sc}}^{0,0}(X, \mathcal{C}) .
$$

Note that $P \in \Psi_{\mathrm{Sc}}^{0,0}(X, \mathcal{C})$ follows from $\psi(H) \in \Psi_{\mathrm{Sc}}^{-\infty, 0}(X, \mathcal{C})$. Thus, $P \geqslant c$, so $P-c^{\prime} \geqslant$ $c-c^{\prime}>0$. Since the spectrum of $P-c^{\prime}$ is a subset of $\left[c-c^{\prime}, \infty\right)$ and $c-c^{\prime}>0$, we have 
$\left(P-c^{\prime}\right)^{1 / 2}=f\left(P-c^{\prime}\right)$ where $f \in \mathcal{C}_{c}^{\infty}(\mathbb{R})$ and $f(t)=\sqrt{t}$ if $t$ is in the spectrum of $P-c^{\prime}$. By Proposition 4.10,

$$
Q=\left(P-c^{\prime}\right)^{1 / 2}=f\left(P-c^{\prime}\right) \in \Psi_{\mathrm{Sc}}^{0,0}(X, \mathcal{C}) .
$$

Let $\psi_{1}$ be identically 1 near supp $\phi$ and vanish near $\operatorname{supp}(1-\psi)$. Then

$$
\psi_{1}(H) Q^{2} \psi_{1}(H)=\psi_{1}(H)\left(P-c^{\prime}\right) \psi_{1}(H)=\psi_{1}(H)\left(A-c^{\prime}\right) \psi_{1}(H) .
$$

Now let $\phi \in \mathcal{C}_{c}^{\infty}(\mathbb{R})$ be identically 1 near $\lambda$ and vanish near $\operatorname{supp}\left(1-\psi_{1}\right)$. Let

$$
B=Q \psi_{1}(H) \in \Psi_{\mathrm{Sc}}^{-\infty, 0}(X, \mathcal{C}) .
$$

Multiplying (8.6) from both sides by $\phi(H)$ then proves (8.3).

We now show that under certain additional assumptions, the positivity of the indicial operators implies positivity of the operator modulo lower order (hence compact) terms in the calculus. We start by assuming strict positivity of the indicial operators when localized in the spectrum of $H$.

Proposition 8.2. - Suppose that $H$ is a many-body Hamiltonian and $\lambda \in \mathbb{R}$. Suppose also that $A, C \in \Psi_{\mathrm{Sc}}^{-\infty, 0}(X, \mathcal{C})$ are self-adjoint and $\hat{C}_{a, 0}(\zeta)=c_{a}(\zeta) \psi_{0}\left(\hat{H}_{a}(\zeta)\right)^{2}$ for every $a$ and $\zeta \in{ }^{\mathrm{sc}} T^{*}\left(\tilde{C}_{a} ; X\right)$ where $c_{a}(\zeta)$ is a function with $c_{a}(\zeta)>0, \psi_{0} \equiv 1$ near $\lambda \in \mathbb{R}, \psi_{0} \in \mathcal{C}_{c}^{\infty}(\mathbb{R})$. Assume in addition that there exists $\psi \in \mathcal{C}_{c}^{\infty}(\mathbb{R})$ which is identically 1 near $\lambda$, $\operatorname{supp} \psi \cap$ $\operatorname{supp}\left(1-\psi_{0}\right)=\emptyset$, such that

$$
\psi\left(\hat{H}_{a}(\zeta)\right) \hat{A}_{a}(\zeta) \psi\left(\hat{H}_{a}(\zeta)\right) \geqslant \psi\left(\hat{H}_{a}(\zeta)\right) c_{a}(\zeta) \psi\left(\hat{H}_{a}(\zeta)\right)
$$

for every $a$ and $\zeta \in{ }^{\mathrm{sc}} T^{*}\left(\tilde{C}_{a} ; X\right)$. Then for any $\varepsilon \in(0,1)$ and $\phi \in \mathcal{C}_{c}^{\infty}(\mathbb{R})$ with

$$
\operatorname{supp} \phi \cap \operatorname{supp}(1-\psi)=\emptyset,
$$

there exists $R \in \Psi_{\mathrm{Sc}}^{-\infty, 1}(X, \mathcal{C})$ such that

$$
\phi(H) A \phi(H) \geqslant(1-\varepsilon) \phi(H) C \phi(H)+R .
$$

Proof. - We apply a parameter dependent version of the previous lemma to the indicial operators to conclude that for each $\zeta$ there exists $\hat{B}_{a}(\zeta)$ with

$$
\phi\left(\hat{H}_{a}(\zeta)\right)\left(\hat{A}_{a}(\zeta)-(1-\varepsilon) \hat{C}_{a}(\zeta)\right) \phi\left(\hat{H}_{a}(\zeta)\right)=\phi\left(\hat{H}_{a}(\zeta)\right) \hat{B}_{a}(\zeta)^{*} \hat{B}_{a}(\zeta) \phi\left(\hat{H}_{a}(\zeta)\right)
$$

It follows from the Cauchy integral formula construction of the square root in the calculus, Remark 4.11, and the explicit formulae (8.4), (8.5) and (8.7) that the indicial operators $\hat{B}_{a}(\zeta)$ match up as discussed before Proposition 4.8, so that there exists $B \in \Psi_{\mathrm{Sc}}^{-\infty, 0}(X, \mathcal{C})$ with indicial operators $\hat{B}_{a}(\zeta)$. Here note that the set where $\psi\left(\hat{H}_{a}(\zeta)\right)$ does not vanish has compact closure, hence $c$ is bounded below on it by a positive constant. Thus, we can take the same smooth function $f$ in the expression (8.5) for the square root for every $a$ and $\zeta$. By (8.11),

$$
\phi(H)(A-(1-\varepsilon) C) \phi(H)=\phi(H) B^{*} B \phi(H)+R
$$

with $R \in \Psi_{\mathrm{Sc}}^{-\infty, 1}(X, \mathcal{C})$. Since $\phi(H) B^{*} B \phi(H) \geqslant 0$, rearranging this proves the proposition.

Similar conclusions hold if we assume a two-sided estimate on the indicial operators of $A$. In essence, this forces the indicial operators, hence their square roots, to vanish to infinite order when $c$ vanishes. 
Proposition 8.3. - Suppose that $H$ is a many-body Hamiltonian and $\lambda \in \mathbb{R}$. Suppose also that $A, C \in \Psi_{\mathrm{Sc}}^{-\infty, 0}(X, \mathcal{C})$ are self-adjoint and $\hat{C}_{a, 0}(\zeta)=c_{a}(\zeta) \psi_{0}\left(\hat{H}_{a}(\zeta)\right)^{2}$ for every $a$ and $\zeta \in{ }^{\operatorname{sc}} T^{*}\left(\tilde{C}_{a} ; X\right)$ where $c_{a}(\zeta)$ is a function with $c_{a}(\zeta) \geqslant 0$ which vanishes with all derivatives at each $\zeta$ with $c_{a}(\zeta)=0, \psi_{0} \equiv 1$ near $\lambda \in \mathbb{R}, \psi_{0} \in \mathcal{C}_{c}^{\infty}(\mathbb{R}), \hat{A}_{a}(\zeta)=0$ if $c_{a}(\zeta)=0$, and for any differential operator $Q \in \operatorname{Diff}\left({ }^{\mathrm{sc}} T^{*}\left(\tilde{C}_{a} ; X\right)\right)$, all seminorms of $Q\left(c_{a}(\zeta)^{-1} \hat{A}_{a}(\zeta)\right)$ in $\Psi_{\mathrm{Sc}}^{-\infty, 0}\left(\rho_{a}^{-1}(p), T_{p} \mathcal{C}^{a}\right), \zeta \in{ }^{\mathrm{sc}} T_{p}^{*}\left(\tilde{C}_{a} ; X\right)$, are uniformly bounded on the set of $\zeta$ 's with $c_{a}(\zeta)>0$. (This is almost, but not quite, a statement about the seminorms of $c_{a}(\zeta)^{-1} \hat{A}_{a}(\zeta)$ in $\Psi_{\mathrm{Sc}, \rho_{a}^{\sharp}}^{-\infty, 0}\left(\rho_{a}^{* \mathrm{sc}} T^{*}\left(\tilde{C}_{a} ; X\right), \tilde{\mathcal{C}}_{a}\right)$, because we restrict our attention to the region where $c_{a}(\zeta)>0$, and do so uniformly.)

Assume in addition that there exists $\psi \in \mathcal{C}_{c}^{\infty}(\mathbb{R})$ which is identically 1 near $\lambda$, $\operatorname{supp} \psi \cap$ $\operatorname{supp}\left(1-\psi_{0}\right)=\emptyset$, such that

$$
\psi\left(\hat{H}_{a}(\zeta)\right) \hat{A}_{a}(\zeta) \psi\left(\hat{H}_{a}(\zeta)\right) \geqslant \psi\left(\hat{H}_{a}(\zeta)\right) c_{a}(\zeta) \psi\left(\hat{H}_{a}(\zeta)\right)
$$

for every a and $\zeta \in{ }^{\mathrm{sc}} T^{*}\left(\tilde{C}_{a} ; X\right)$. Then the conclusion of the previous proposition holds, i.e. for any $\varepsilon \in(0,1)$ and $\phi \in \mathcal{C}_{c}^{\infty}(\mathbb{R})$ with

$$
\operatorname{supp} \phi \cap \operatorname{supp}(1-\psi)=\emptyset,
$$

there exists $R \in \Psi_{\mathrm{Sc}}^{-\infty, 1}(X, \mathcal{C})$, with seminorms bounded by those of $A$ and $C$ in $\Psi_{\mathrm{Sc}}^{-\infty, 0}(X, \mathcal{C})$, and with $\mathrm{WF}_{\mathrm{Sc}}^{\prime}(R) \subset \mathrm{WF}_{\mathrm{Sc}}^{\prime}(A) \cup \mathrm{WF}_{\mathrm{Sc}}^{\prime}(C)$ such that

$$
\phi(H) A \phi(H) \geqslant(1-\varepsilon) \phi(H) C \phi(H)+R .
$$

Proof. - We define $\hat{B}_{a}(\zeta)=0$ if $c_{a}(\zeta)=0$, otherwise we define $\hat{B}_{a}(\zeta)$ as in the previous proposition. The only additional ingredient is the analysis of $\hat{B}_{a}(\zeta)$ near $\zeta$ with $c_{a}(\zeta)=0$. To do this analysis, we follow the construction of $\hat{B}_{a}(\zeta)$ in detail. So let

$$
\hat{P}_{a}(\zeta)=\psi\left(\hat{H}_{a}(\zeta)\right) \hat{A}_{a}(\zeta) \psi\left(\hat{H}_{a}(\zeta)\right)+c_{a}(\zeta)\left(\operatorname{Id}-\psi\left(\hat{H}_{a}(\zeta)\right)^{2}\right)
$$

and let

$$
c_{a}^{\prime}(\zeta)=(1-\varepsilon) c_{a}(\zeta)
$$

Thus, $\hat{P}_{a}(\zeta)-c_{a}^{\prime}(\zeta) \geqslant \varepsilon c_{a}(\zeta)$. Let

$$
\hat{Q}_{a}(\zeta)=\left(\hat{P}_{a}(\zeta)-c_{a}^{\prime}(\zeta)\right)^{1 / 2}=c_{a}(\zeta)^{1 / 2}\left(c_{a}(\zeta)^{-1} \hat{P}_{a}(\zeta)-(1-\varepsilon)\right)^{1 / 2}
$$

By our assumption, there exists $M>0$ such that the norm of $\hat{P}_{a}(\zeta)$ in $\mathcal{B}\left(L^{2}, L^{2}\right)$ is bounded by $M c_{a}(\zeta)$. Now choose $f \in \mathcal{C}_{c}^{\infty}(\mathbb{R})$ such that $f(t)=\sqrt{t}$ on $[1-\varepsilon, M]$. Then $M \geqslant c_{a}(\zeta)^{-1} \hat{P}_{a}(\zeta)-$ $1+\varepsilon \geqslant \varepsilon$, so

$$
\hat{Q}_{a}(\zeta)=c_{a}(\zeta)^{1 / 2} f\left(c_{a}(\zeta)^{-1} \hat{P}_{a}(\zeta)-(1-\varepsilon)\right) .
$$

By our assumptions, the seminorms of $c_{a}(\zeta)^{-1} \hat{P}_{a}(\zeta)$ in $\Psi_{\mathrm{Sc}}^{0,0}\left(\rho_{a}^{-1}(p), T_{p} \mathcal{C}^{a}\right), \zeta \in{ }^{\mathrm{sc}} T_{p}^{*}\left(\tilde{C}_{a} ; X\right)$, remain uniformly bounded as $c_{a}(\zeta) \rightarrow 0$, so the Cauchy integral representation of $f$, via an almost analytic extension, shows that $f\left(c_{a}(\zeta)^{-1} \hat{P}_{a}(\zeta)-(1-\varepsilon)\right)$ remains uniformly bounded. Thus, $\hat{Q}_{a}(\zeta)$ is continuous as a function on ${ }^{\mathrm{sc}} T^{*}\left(\tilde{C}_{a} ; X\right)$ with values in $\Psi_{\mathrm{Sc}}^{0,0}\left(\rho_{a}^{-1}(p), T_{p} \mathcal{C}^{a}\right)$. A similar argument also holds for the derivatives of $\hat{Q}_{a}(\zeta)$. Let $\psi_{1}$ be identically 1 near $\operatorname{supp} \phi$ 
and vanish near $\operatorname{supp}(1-\psi)$, and let

$$
\hat{B}_{a}(\zeta)=\hat{Q}_{a}(\zeta) \psi_{1}(H) .
$$

Again, the $\hat{B}_{a}(\zeta)$ match up so there exists $B \in \Psi_{\mathrm{Sc}}^{-\infty, 0}(X, \mathcal{C})$ with these indicial operators. We can also make sure that the lower order terms also vanish where $c$ does, i.e. that $\mathrm{WF}_{\mathrm{Sc}}^{\prime}(B) \subset$ $\operatorname{supp} c$. Then the indicial operators of $\phi(H)(A-(1-\varepsilon) C) \phi(H)$ and $\phi(H) B^{*} B \phi(H)$ are the same, so

$$
\phi(H)(A-(1-\varepsilon) C) \phi(H)=\phi(H) B^{*} B \phi(H)+R
$$

with $R \in \Psi_{\mathrm{Sc}}^{-\infty, 1}(X, \mathcal{C})$, proving the proposition.

\section{Commutators}

In this section we discuss the basic technical tool underlying the propagation estimates of the following sections. Thus, we show how an estimate of the commutator $[A, H]$ at $\tilde{C}_{0}$, which is essentially obtained by a symbolic calculation in the scattering calculus, can give a positive commutator estimate under the additional assumption that $\hat{H}_{a, 0}(\zeta)$ has no $L^{2}$ eigenfunctions for any $a \neq 0$ and $\zeta \in{ }^{\mathrm{sc}} T^{*}\left(\tilde{C}_{a} ; X\right)$. In the Euclidean setting this means simply that the subsystems have no bound states.

To do so, we extend the notion of a function being $\pi$-invariant to functions on ${ }^{\mathrm{sc}} T^{*} X$ in a trivial way: $q \in \mathcal{C}^{\infty}\left({ }^{\mathrm{sc}} T^{*} X\right)$ is $\pi$-invariant if $\left.q\right|_{{ }^{s c}} T_{\partial X}^{*} X$ is $\pi$-invariant. Since the analysis of classical dynamics, i.e. of generalized broken bicharacteristics of $\Delta-\lambda$, broken at $\mathcal{C}$, is based on the properties of $\pi$-invariant functions, we will be interested in quantizing $\pi$-invariant symbols. More specifically, we are essentially interested in operators of the form $A=Q \psi_{0}(H), \psi_{0} \in \mathcal{C}_{c}^{\infty}(\mathbb{R})$, where $Q$ is obtained by quantizing a $\pi$-invariant function $q \in \mathcal{C}^{\infty}\left({ }^{\mathrm{sc}} T^{*} X\right)$. Since such $Q$ would not be in our calculus, we construct $A$ directly.

All considerations in what follows will be local, i.e. we will assume that the projection of the support of $q$ to $X$ lies near a fixed $p \in \partial X$, so we can always work in local coordinates and identify $X$ with $\mathbb{S}_{+}^{n}$. The problem with such $q \in \mathcal{C}^{\infty}\left(\mathbb{S}_{+}^{n} \times \mathbb{R}^{n}\right)$ is that they are rarely in $\mathcal{C}^{\infty}\left(\mathbb{S}_{+}^{n} \times \mathbb{S}_{+}^{n}\right)$, i.e. they are not symbols in $\xi$, so $Q$ will not be in $\Psi_{\mathrm{sc}}^{0,0}\left(\mathbb{S}_{+}^{n}\right)$ or indeed in $\Psi_{\mathrm{Sc}}^{0,0}\left(\mathbb{S}_{+}^{n}, \mathcal{C}\right)$. This, however, is not a major difficulty. Fix $\psi_{0} \in \mathcal{C}_{c}^{\infty}(\mathbb{R} ;[0,1])$ which is identically 1 in a neighborhood of a fixed $\lambda$. Thus, $\psi_{0}(H) \in \Psi_{\mathrm{Sc}}^{-\infty, 0}(X, \mathcal{C})$, so it is smoothing. At the symbol level, $\psi_{0}(H)$ is locally the right quantization of some

$$
p \in \mathcal{C}^{\infty}\left(\left[\mathbb{S}_{+}^{n} ; \mathcal{C}\right] \times \mathbb{S}_{+}^{n}\right)
$$

which vanishes to infinite order at $\left[\mathbb{S}_{+}^{n} ; \mathcal{C}\right] \times \partial \mathbb{S}_{+}^{n}$, which will enable us to write down $A$ directly.

We are thus interested in the following class of symbols $q$. We assume that $q \in \mathcal{C}^{\infty}\left(\mathbb{R}_{w}^{n} \times \mathbb{R}_{\xi}^{n}\right)$ and that for every multiindex $\alpha, \beta \in \mathbb{N}^{n}$ there exist constants $C_{\alpha, \beta}$ and $m_{\alpha, \beta}$ such that

$$
\left|\left(D_{w}^{\alpha} D_{\xi}^{\beta} q\right)(w, \xi)\right| \leqslant C_{\alpha, \beta}\langle w\rangle^{-|\alpha|}\langle\xi\rangle^{m_{\alpha, \beta}} .
$$

This implies, in particular, that

$$
q \in \mathcal{A}^{0}\left(\mathbb{S}_{+}^{n} \times \mathbb{R}^{n}\right)
$$

i.e. that $q$ is a 0 th order symbol in $w$, though it may blow up polynomially in $\xi$. Indeed, in the compactified notation, (9.2) becomes that for every $P \in \operatorname{Diff}_{\mathrm{b}}\left(\mathbb{S}_{+}^{n}\right)$, acting in the base $(w)$ 
variables, and for every $\beta \in \mathbb{N}^{n}$ there exist $C_{P, \beta}$ and $m_{P, \beta}$ such that

$$
\left|\left(P D_{\xi}^{\beta}\right) q\right| \leqslant C_{P, \beta}\langle\xi\rangle^{m_{P, \beta}} .
$$

It is convenient to require that $q$ be polyhomogeneous on $\mathbb{S}_{+}^{n} \times \mathbb{R}^{n}$ :

$$
q \in \mathcal{C}^{\infty}\left(\mathbb{S}_{+}^{n} \times \mathbb{R}^{n}\right)
$$

this stronger statement automatically holds for the $\pi$-invariant symbols we are interested in.

We next introduce the product symbol

$$
a\left(w, w^{\prime}, \xi\right)=q(w, \xi) p\left(w^{\prime}, \xi\right),
$$

where $\psi_{0}(H)$ is given locally by the right quantization of $p$. The main point is

LEMMA 9.1. - The symbol a defined by (9.6) is in $\mathcal{C}^{\infty}\left(\mathbb{S}_{+}^{n} \times\left[\mathbb{S}_{+}^{n} ; \mathcal{C}\right] \times \mathbb{S}_{+}^{n}\right)$ and it vanishes with all derivatives at $\left[\mathbb{S}_{+}^{n} ; \mathcal{C}\right] \times \partial \mathbb{S}_{+}^{n}$. Hence, it defines an operator $A \in \Psi_{\mathrm{Sc}}^{-\infty, 0}(X, \mathcal{C})$ by the oscillatory integral (3.16).

Proof. - First, $a \in \mathcal{C}^{\infty}\left(\mathbb{S}_{+}^{n} \times\left[\mathbb{S}_{+}^{n} ; \mathcal{C}\right] \times \mathbb{R}^{n}\right)$ follows from (9.1) and (9.5). Moreover, the infinite order vanishing of $p$ at $\left[\mathbb{S}_{+}^{n} ; \mathcal{C}\right] \times \mathbb{S}^{n-1}$ implies that for every $P^{\prime} \in \operatorname{Diff}_{\mathrm{b}}\left(\left[\mathbb{S}_{+}^{n} ; \mathcal{C}\right]\right), \beta \in \mathbb{N}^{n}$ and $N \in \mathbb{N}$,

$$
\left|P^{\prime} D_{\xi}^{\beta} p\right| \leqslant C_{P^{\prime}, \beta, N}\langle\xi\rangle^{-N} .
$$

Thus, Leibniz' rule shows that for $P \in \operatorname{Diff}_{\mathrm{b}}\left(\mathbb{S}_{+}^{n}\right)$ acting in $w, P^{\prime} \in \operatorname{Diff}_{\mathrm{b}}\left(\left[\mathbb{S}_{+}^{n} ; \mathcal{C}\right]\right)$ acting in $w^{\prime}$, $\beta \in \mathbb{N}^{n}$ and $N$

$$
\left|P P^{\prime} D_{\xi}^{\beta} a\right| \leqslant C_{P P^{\prime}, \beta, N}\langle\xi\rangle^{-N} .
$$

But this means precisely that $a \in \mathcal{C}^{\infty}\left(\mathbb{S}_{+}^{n} \times\left[\mathbb{S}_{+}^{n} ; \mathcal{C}\right] \times \mathbb{S}_{+}^{n}\right)$ and it vanishes to infinite order at the boundary in the last factor.

The indicial operators of $A$ are just given by the quantization of the appropriate restriction of $a$ similarly to (4.48) (except that now $a$ depends on the base variables from both the left and the right factors of $\mathbb{S}_{+}^{n}$ ). This takes a particularly simple form if $q$ is $\pi$-invariant, for then, in the notation of (4.48), $q$ is independent of both $Y$ and $\xi^{a}$. Thus, we can take $q$ outside the integral in (4.48), i.e. it simply multiplies the indicial operator of $\psi_{0}(H)$ by a constant.

Lemma 9.2. - Suppose that $q \in \mathcal{C}^{\infty}\left({ }^{\mathrm{sc}} T^{*} \mathbb{S}_{+}^{n}\right)$ is $\pi$-invariant and it satisfies (9.4). Let $A \in \Psi_{\mathrm{Sc}}^{-\infty, 0}(X, \mathcal{C})$ be as in the previous lemma. If $\zeta \in{ }^{\mathrm{sc}} T^{*}\left(\tilde{C}_{a} ; X\right)$, then $\hat{A}_{a}(\zeta)=q(\zeta) \widehat{\psi_{0}(H)_{a}}(\zeta)$.

Combining this lemma with Proposition 5.5 gives

Corollary 9.3. - Suppose that $\zeta \in{ }^{\mathrm{sc}} T_{C_{a}^{\prime}}^{*}\left(C_{a} ; X\right)$ and $u \in \mathcal{C}^{-\infty}(X)$. If $A$ is as in Lemma 9.2, $q(\zeta) \neq 0, A u \in \dot{\mathcal{C}}^{\infty}(X)$ and $\zeta \notin \mathrm{WF}_{\mathrm{Sc}}((H-\lambda) u)$ then $\zeta \notin \mathrm{WF}_{\mathrm{Sc}}(u)$.

Since the indicial operator of $[A, H]=A H-H A$ in $\Psi_{\mathrm{Sc}}^{-\infty, 0}(X, \mathcal{C})$ is just

$$
\widehat{[A, H}]_{a, 0}(\zeta)=\left[\hat{A}_{a, 0}(\zeta), \hat{H}_{a, 0}(\zeta)\right]=q(\zeta)\left[\psi_{0}\left(\hat{H}_{a, 0}(\zeta)\right), \hat{H}_{a, 0}(\zeta)\right]=0
$$

for every $a$ and $\zeta \in{ }^{\mathrm{sc}} T^{*}\left(\tilde{C}_{a} ; X\right)$, we see that for every $A$ as in Lemma 9.1, $[A, H] \in$ $\Psi_{\mathrm{Sc}}^{-\infty, 1}(X, \mathcal{C})$. The additional order of decay corresponds to the one in the scattering calculus. 
Moreover, the indicial operator of $[A, H]$ at $\tilde{C}_{0}$, as an operator in $\Psi_{\mathrm{Sc}}^{-\infty, 1}(X, \mathcal{C})$ (so this indicial operator is just a function on $\left.{ }^{\mathrm{sc}} T^{*}\left(\tilde{C}_{0} ; \underset{\tilde{C}}{X}\right)\right)$, is given by the Poisson bracket formula from the scattering calculus. Since $V$ vanishes at $\tilde{C}_{0}$, this gives

$$
\widehat{i[A, H}]_{1,0}=-{ }^{\mathrm{sc}} H_{g}\left(q \psi_{0}(g)\right)=-\psi_{0}(g)^{\mathrm{sc}} H_{g} q .
$$

If the indicial operators of $H$ at the other faces have no $L^{2}$ eigenfunctions, then this estimate combined with a compactness argument suffices to prove an estimate for $[A, H]$ modulo lower operators (i.e. modulo $\Psi_{\mathrm{Sc}}^{-\infty, 2}(X, \mathcal{C})$ ). However, to make the compactness argument work, we need to estimate the indicial operators, $\widehat{[A, H}]_{a, 1}$, for all $a$. This is facilitated by the following lemma.

LEMMA 9.4. - Let $q$ and $A$ be as in Lemma 9.2. For every seminorm in

$$
\Psi_{\mathrm{Sc}}^{-\infty, 0}\left(\rho_{a}^{-1}(p), T_{p} \mathcal{C}^{a}\right)
$$

and for every $l \in \mathbb{N}$ there exist $C>0$ and $m \in \mathbb{N}$ such that for every a and every $\zeta \in{ }^{\mathrm{sc}} T_{p}^{*}\left(\tilde{C}_{a} ; X\right), p \in \tilde{C}_{a}$, the seminorm of $\left.\widehat{[A, H}\right]_{a, 1}(\zeta)$ in $\Psi_{\mathrm{Sc}}^{-\infty, 0}\left(\rho_{a}^{-1}(p), T_{p} \mathcal{C}^{a}\right)$ is bounded by

$$
C\left(|q(\zeta)|+\sum_{|\alpha| \leqslant m} \sup _{\xi^{a}}\left|\left\langle\xi^{a}\right\rangle^{-l}\left(\partial_{\xi^{a}}^{\alpha} d q\right)\left(\zeta, \xi^{a}\right)\right|\right)
$$

where the differential dq is taken with respect to all variables, in ${ }^{\mathrm{sc}} T^{*} \mathbb{S}_{+}^{n}$, i.e. it is the differential of $q \in \mathcal{C}^{\infty}\left({ }^{\mathrm{sc}} T^{*} \mathbb{S}_{+}^{n}\right)$.

Remark 9.5. - Similar conclusions hold for every seminorm in $\Psi_{\mathrm{Sc}, \rho_{a}^{\sharp}}^{-\infty}\left(\rho_{a}^{* \mathrm{sc}} T^{*}\left(\tilde{C}_{a} ; X\right), \tilde{\mathcal{C}}_{a}\right)$, which can be seen directly from our calculations in the following proof.

Proof. - This can be proved directly from the definition of the indicial operators, i.e. by computing $x^{-1} \mathrm{e}^{-i \tilde{f}}[A, H] \mathrm{e}^{i \tilde{f}} u^{\prime}$ where $\tilde{f} \in \mathcal{C}^{\infty}(X)$ and $u^{\prime} \in \mathcal{C}^{\infty}([X ; \mathcal{C}])$, similarly to [40, Sections 7,13]. Since this is equal to $x^{-1}\left[\mathrm{e}^{-i \tilde{f}} A \mathrm{e}^{i \tilde{f}}, \mathrm{e}^{-i \tilde{f}} H \mathrm{e}^{i \tilde{f}}\right] u^{\prime}$, and $\mathrm{e}^{-i \tilde{f}} A \mathrm{e}^{i \tilde{f}} \in$ $\Psi_{\mathrm{Sc}}^{-\infty, 0}(X, \mathcal{C})$, we can assume that $\tilde{f}=0$, the calculation being very similar in the general case. To compute the commutator, it suffices to commute both $A v$ and $H v$ for every $v \in \mathcal{C}^{\infty}([X ; \mathcal{C}])$ modulo terms that vanish with their first derivatives in $\beta_{\mathrm{Sc}}^{*} C_{a}$. A straightforward calculation can be performed just as in (4.42)-(4.48), where only the 0th order terms were kept. That shows with our coordinates that

$$
\begin{aligned}
\widehat{[A, H}_{a, 1}(\zeta)= & {\left[{\widehat{\left(\partial_{x} A\right.}}_{a, 0}(\zeta), \hat{H}_{a, 0}(\zeta)\right] } \\
& +\left(-\left(D_{\tau} q\right)(\zeta)\left(\left[Y, \hat{H}_{a, 0}(\zeta)\right] \partial_{Y}+Y \partial_{Y} \hat{H}_{a, 0}(\zeta)\right)\right. \\
& +\left(D_{\nu} q\right)(\zeta)\left(\partial_{z} \hat{H}_{a, 0}\right)(\zeta)-\left(\partial_{z} q\right)(\zeta)\left(D_{\nu} \hat{H}_{a, 0}\right)(\zeta) \\
& \left.+\left(\partial_{\tau} q\right)(\zeta)\left(\nu \cdot D_{\nu} \hat{H}_{a, 0}\right)(\zeta)-\left(\nu \cdot D_{\nu} q\right)(\zeta)\left(\partial_{\tau} \hat{H}_{a, 0}\right)\right) \psi\left(\hat{H}_{a, 0}(\zeta)\right)
\end{aligned}
$$

Here $\partial_{x} A$ denotes the operator with kernel given by $\partial_{x}$ applied to that of $A$. Since in our notation the kernel of $A$ is

$$
\int \mathrm{e}^{i\left(w-w^{\prime}\right) \cdot \xi} q(w, \xi) p\left(w^{\prime}, \xi\right) d \xi
$$

with the integral being convergent, rewriting this with the coordinates on the compactification $\left[\mathbb{S}_{+}^{n} ; \mathcal{C}_{a}\right],(2.7)$, so that $q$ takes the form $q(x, x Y, z, \xi)$ proves that all terms of (9.12) satisfy the stated estimate, completing the proof. 
Another approach to compute $a$-indicial operators is to use that near $C_{a}^{\prime}, A$ can be regarded as a (non-classical!) pseudo-differential operator in the free variables $\left(w_{a}, \xi_{a}\right)$ with values in bounded operators on $L^{2}\left(X_{a}\right)$ (in fact, with values in $\Psi_{\mathrm{Sc}}^{-\infty, 0}\left(\bar{X}^{a}, \mathcal{C}^{a}\right)$ ). More precisely, $A \in \Psi_{\mathrm{scc}}^{-\infty, 0}\left(\bar{X}_{a} ; \mathcal{B}\left(L^{2}\left(X^{a}\right), L^{2}\left(X^{a}\right)\right)\right)$. This allows us to use the scattering calculus for the computation of the commutators to give the stated result.

As an application of these estimates, we now show how, under the assumption that the subsystems have no bound states, a positive Poisson bracket with $g$ can give rise to a positive operator estimate. We thus assume that

$$
\hat{H}_{a, 0}(\xi) \text { has no } L^{2} \text { eigenvalues for any } a \neq 0 \text { and } \zeta \in{ }^{\mathrm{sc}} T^{*}\left(\tilde{C}_{a} ; X\right) .
$$

To simplify the notation in the following proposition, we introduce the notation $\operatorname{supp}_{a} e \subset$ ${ }^{\mathrm{sc}} T^{*}\left(\tilde{C}_{a} ; X\right)$ for $\pi$-invariant functions $e \in \mathcal{C}^{\infty}\left({ }^{\mathrm{sc}} T_{\partial X}^{*} X\right)$. This is defined as the support of the function on ${ }^{\mathrm{sc}} T^{*}\left(\tilde{C}_{a} ; X\right)$ induced by $e$. Indeed, as $e$ is $\pi$-invariant, its restriction to ${ }^{\mathrm{sc}} T_{C_{a}}^{*} X$ can be regarded as a function on ${ }^{\mathrm{sc}} T^{*}\left(C_{a} ; X\right)$. Then $\operatorname{supp}_{a} e$ is the support of the pull-back of this function to ${ }^{\mathrm{sc}} T^{*}\left(\tilde{C}_{a} ; X\right)$.

Proposition 9.6. - Suppose that $H$ is a many-body Hamiltonian satisfying (9.14), and $\lambda \in \mathbb{R}$. Suppose also that $q, b, e \in \mathcal{C}^{\infty}\left({ }^{\mathrm{sc}} T^{*} X ; \mathbb{R}\right)$ are $\pi$-invariant, satisfy the bounds (9.4), $q, b \geqslant 0$, and that there exist $\delta>0, C>0, C_{\alpha}>0$, such that for all $\xi \in{ }^{\mathrm{sc}} T_{\partial X}^{*} X$,

$$
|g(\xi)-\lambda|<\delta \Rightarrow{ }^{\mathrm{sc}} H_{g} q(\xi) \leqslant-b(\xi)^{2}+e(\xi)
$$

and

$$
|g(\xi)-\lambda|<\delta \quad \text { and } \quad \xi \notin \operatorname{supp} e \Rightarrow q(\xi) \leqslant C b(\xi)^{2} \quad \text { and } \quad\left|\left(\partial_{\mu}^{\alpha} d q\right)(\xi)\right| \leqslant C_{\alpha} b(\xi)^{2} .
$$

Let $A \in \Psi_{\mathrm{Sc}}^{-\infty, 0}(X, \mathcal{C})$ be as in Lemma 9.1. For any $\varepsilon^{\prime}>0, a \in I$ and for any $K_{a} \subset{ }^{\mathrm{sc}} T^{*}\left(\tilde{C}_{a} ; X\right)$ compact with $\operatorname{supp}_{a} e \cap K=\emptyset$ there exists $\delta^{\prime}>0$ such that if $\psi \in \mathcal{C}_{c}^{\infty}(\mathbb{R})$ is supported in $\left(\lambda-\delta^{\prime}, \lambda+\delta^{\prime}\right)$ and $\zeta \in K_{a}$ then

$$
i\left(\psi(H)\left[\widehat{A^{*} A, H}\right] \psi(H)\right)_{a, 1}(\zeta) \geqslant\left(2-\varepsilon^{\prime}\right) b^{2} q \psi\left(\hat{H}_{a, 0}(\zeta)\right)^{2} .
$$

Proof. - Note that the estimate (9.17) is trivial if $\tau_{a}^{2}+\left|\nu_{a}\right|_{z_{a}}^{2}>\lambda+1$ (with $\zeta=\left(z_{a}, \tau_{a}, \nu_{a}\right)$, $\delta^{\prime}<1$ arbitrary) since then both sides vanish as

$$
\widehat{\psi(H)}_{a}(\zeta)=\psi\left(h_{a}\left(z_{a}\right)+\tau_{a}^{2}+\left|\nu_{a}\right|_{z_{a}}^{2}\right)
$$

$h_{a}$ denoting the subsystem Hamiltonian as in (6.9), and $H_{a} \geqslant 0$ by the assumption on the absence of bound states of all subsystem Hamiltonians (including $H_{c}$ with $\left.C_{a} \subset C_{c}\right)$. $\left(H_{a} \geqslant 0\right.$ is really an HVZ-type result: it follows inductively by Proposition 4.8 that one can construct a parametrix for $H_{a}-\sigma, \sigma<0$, and then the absence of bound states implies invertibility on $L^{2}$, so the spectrum of $H_{a}$ is disjoint from $(-\infty, 0)$.)

We prove (9.17) by induction on $a$. First, (9.17) is certainly satisfied for $a=0$. In fact, as $A \in \Psi_{\mathrm{sc}}^{-\infty, 0}(X, \mathcal{C})$, we can use the commutator formula in the scattering calculus, (9.10), to find $\widehat{[A, H}]_{0,1}$. Since $V$ vanishes at the free face, $\beta_{\mathrm{Sc}}^{*} C_{0}$, it does not contribute to $\left.\widehat{[A, H}\right]_{0,1}$, so we indeed have, by (9.15),

$$
i \psi(H)\left[\widehat{A^{*} A, H}\right] \psi(H)_{0,1}=-2 q\left({ }^{\mathrm{sc}} H_{g} q\right) \psi(g)^{2} \geqslant 2 b^{2} q \psi(g)^{2}=2 b^{2} q \psi\left(\hat{H}_{0,0}\right)^{2}
$$


away from $\operatorname{supp}_{0} e$ under the assumption that

$$
\operatorname{supp} \psi \subset(\lambda-\delta, \lambda+\delta) \text {. }
$$

So suppose now that (9.17) has been proved for all $c$ with $C_{a} \subset C_{c}, C_{a} \neq C_{c}$. This implies that all indicial operators of $i\left[\psi(H) \widehat{A^{*} A \psi}(H), H\right]_{a}(\zeta), \zeta=\left(z_{a}, \tau_{a}, \nu_{a}\right) \in K_{a}$ satisfy an inequality like (9.17). In fact, the indicial operators are of the form $i\left[\psi(H) \widehat{A^{*} A \psi}(H), H\right]_{c}(\tilde{\zeta})$ with $\beta_{\mathrm{Sc}}(\tilde{\zeta})=\left(0, z_{a}\right) \in C_{a}, \tilde{\pi}_{c a}(\tilde{\zeta})=\zeta$. Such a $\tilde{\zeta}$ is of the form $\tilde{\zeta}=\left(\hat{Y}_{a}^{\prime \prime}, z_{a}, \tau_{a}, \mu_{a}^{\prime \prime}, \nu_{a}\right)$ where $C_{c}$ is given by $x=0, y^{\prime}=0$, so $\left(\hat{Y}_{a}^{\prime \prime}, z_{a}\right)$ give coordinates along $\tilde{C}_{c}$. Note that as $K_{a}$ is compact, so is

$$
K_{c}=\left\{\tilde{\zeta}=\left(\hat{Y}_{a}^{\prime \prime}, z_{a}, \tau_{a}, \mu_{a}^{\prime \prime}, \nu_{a}\right):\left(z_{a}, \tau_{a}, \nu_{a}\right) \in K_{a}, \beta_{\mathrm{Sc}}(\tilde{\zeta}) \in C_{a},\left|\mu_{a}^{\prime \prime}\right| \leqslant \lambda+1\right\}
$$

and as $e$ is independent of $\mu_{a}^{\prime \prime}$ at $C_{a}, K_{c} \cap \operatorname{supp}_{c} e=\emptyset$, so we can apply the inductive hypothesis. Taking into account that the estimate (9.17) is trivial at $C_{c}$ for $\tilde{\zeta}$ with $\left|\mu_{a}^{\prime \prime}\right|>\lambda+1$, we see that for all $\tilde{\zeta}=\left(0, z_{a}, \tau_{a}, \mu_{a}^{\prime \prime}, \nu_{a}\right)$ with $\left(z_{a}, \tau_{a}, \nu_{a}\right) \in K_{a}$, we have

$$
i\left(\psi(H)\left[\widehat{A^{*} A, H}\right] \psi(H)\right)_{c, 1}(\tilde{\zeta}) \geqslant\left(2-\varepsilon^{\prime}\right) b^{2} q \widehat{\psi(H)^{2}}{ }_{c, 0}(\tilde{\zeta}) .
$$

Since $b^{2} q$ is $\pi$-invariant on ${ }^{\mathrm{sc}} T^{*} X$, it is independent of $\tilde{\zeta}$ for each fixed $\zeta$, and if it vanishes at $\zeta$, then so does $\left[\psi(H) \widehat{A^{*} A \psi}(H), H\right]_{a, 1}(\zeta)$ by Lemmas 9.2-9.4 and (9.16). Thus, by Proposition 8.2,

$$
i\left[\psi(H) \widehat{A^{*} A \psi}(H), H\right]_{a, 1}(\zeta) \geqslant\left(2-\varepsilon^{\prime}\right) b^{2} q \widehat{\psi(H)}_{a, 0}^{2}(\zeta)+R(\zeta)
$$

where the seminorms of

$$
R(\zeta) \in \Psi_{\mathrm{Sc}}^{-\infty, 1}\left(\rho_{a}^{-1}(p), T_{p} \mathcal{C}^{a}\right), \quad \zeta \in{ }^{\mathrm{sc}} T_{p}^{*}\left(\tilde{C}_{a} ; X\right),
$$

are bounded by those of $\left[\psi(H) \widehat{A^{*} A \psi}(H), H\right]_{a, 1}(\zeta)$ and by $b(\zeta)^{2} q(\zeta)$. By assumption (9.16) and Lemma 9.4 the former are bounded by the latter, so $R(\zeta)$ satisfies the estimate

$$
\|R(\zeta)\|_{\mathcal{B}\left(L_{\mathrm{sc}}^{2}\left(\rho_{a}^{-1}(p)\right), H_{\mathrm{sc}}^{1,1}\left(\rho_{a}^{-1}(p)\right)\right)} \leqslant C^{\prime \prime} q(\zeta) b(\zeta)^{2}
$$

with $C^{\prime \prime}$ independent of $q$ and $b$.

We now use our hypothesis on the absence of bound states. So suppose that $\psi_{1}, \psi_{2} \in \mathcal{C}_{c}^{\infty}(\mathbb{R})$, $\psi \equiv 1$ near $\operatorname{supp} \psi_{1}, \psi_{1} \equiv 1$ near $\operatorname{supp} \psi_{2}$. By assumption, $\lambda-\tau_{a}^{2}-\left|\nu_{a}\right|_{z_{a}}^{2}$ is not an eigenvalue of the subsystem Hamiltonian, $h_{a}(z)$. Thus,

$$
\psi_{1}\left(\hat{H}_{a}(\zeta)\right)=\psi_{1}\left(h_{a}(z)+\tau_{a}^{2}+\left|\nu_{a}\right|_{z_{a}}^{2}\right) \rightarrow 0
$$

strongly as $\operatorname{supp} \psi_{1} \rightarrow\{\lambda\}$. Since $K_{a}$ is compact, and the inclusion map

$$
T: H_{\mathrm{sc}}^{1,1}\left(\rho_{a}^{-1}(p)\right) \hookrightarrow L_{\mathrm{sc}}^{2}\left(\rho_{a}^{-1}(p)\right)
$$

is compact, for $\psi_{1}$ with sufficiently small support we have

$$
\left\|\left(\widehat{\psi_{1}(H) T}\right)_{a}(\zeta)\right\|_{\mathcal{B}\left(H_{\mathrm{sc}}^{1,1}\left(\rho_{a}^{-1}(p)\right), L_{\mathrm{sc}}^{2}\left(\rho_{a}^{-1}(p)\right)\right)} \leqslant \varepsilon^{\prime}\left(C^{\prime \prime}\right)^{-1}
$$


for all $\zeta \in K_{a}$. Thus,

$$
i\left(\psi_{1}(H)\left[\widehat{A^{*} A, H}\right] \psi_{1}(H)\right)_{a, 1}(\zeta) \geqslant\left(2-\varepsilon^{\prime}\right) b^{2} q{\widehat{\psi_{1}(H)}}_{a, 0}^{2}(\zeta)-\varepsilon^{\prime} b^{2} q, \quad \zeta \in K_{a}
$$

Multiplying by $\psi_{2}(H)$ from both left and right we finally conclude that

$$
i\left(\psi_{2}(H)\left[\widehat{A^{*} A, H}\right] \psi_{2}(H)\right)_{a, 1} \geqslant\left(2-2 \varepsilon^{\prime}\right) b^{2} q{\widehat{\psi_{2}(H)}}_{a, 0}^{2} .
$$

Relabelling $\psi_{2}$ and $2 \varepsilon^{\prime}$ as $\psi$ and $\varepsilon^{\prime}$ (thereby putting stronger restrictions on $\operatorname{supp} \psi$ ) provides the inductive step and completes the proof of (9.17).

In the following corollary we add an extra term to the commutator that will enable us to deal with other terms arising in the propagation estimates.

COROLlary 9.7. - Suppose that the assumptions of Proposition 9.6 are satisfied and let $C$ be as in (9.16). Suppose in addition that for any differential operator $Q$ on ${ }^{\mathrm{sc}} T^{*}\left(\tilde{C}_{a} ; X\right)$ and multiindex $\alpha$ there exist constant $C_{Q}$ and $C_{\alpha, Q}$ such that

$$
\begin{aligned}
& |g(\xi)-\lambda|<\delta, b(\xi) \neq 0 \quad \text { and } \quad \xi \notin \operatorname{supp} e \\
& \quad \Rightarrow\left|Q\left(b^{-2} q\right)(\xi)\right| \leqslant C_{Q} \quad \text { and }\left|Q\left(b^{-2}\left(\partial_{\mu}^{\alpha} d q\right)\right)(\xi)\right| \leqslant C_{\alpha, Q} .
\end{aligned}
$$

For any $\varepsilon^{\prime}>0, M>0$, and for any $K \subset{ }^{\mathrm{sc}} \dot{T}^{*} X$ compact with supp $e \cap K=\emptyset$ there exist $\delta^{\prime}>0$, $B, E \in \Psi_{\mathrm{Sc}}^{-\infty, 0}(X, \mathcal{C}), F \in \Psi_{\mathrm{Sc}}^{-\infty, 1}(X, \mathcal{C})$ with

$$
\begin{aligned}
& \mathrm{WF}_{\mathrm{Sc}}^{\prime}(E) \cap K=\emptyset, \quad \mathrm{WF}_{\mathrm{Sc}}^{\prime}(F) \subset \operatorname{supp} q, \\
& \hat{B}_{a, 0}(\zeta)=b(\zeta) q(\zeta)^{1 / 2} \psi\left(\hat{H}_{a, 0}(\zeta)\right), \quad \zeta \in K,
\end{aligned}
$$

such that if $\psi \in \mathcal{C}_{c}^{\infty}(\mathbb{R})$ is supported in $\left(\lambda-\delta^{\prime}, \lambda+\delta^{\prime}\right)$ then

$$
\begin{aligned}
& i \psi(H) x^{-1 / 2}\left[A^{*} A, H\right] x^{-1 / 2} \psi(H)-M \psi(H) A^{*} A \psi(H) \\
& \quad \geqslant\left(2-\varepsilon^{\prime}-M C\right) B^{*} B+E+F .
\end{aligned}
$$

Proof. - Let $p \in \mathcal{C}^{\infty}\left({ }^{\mathrm{sc}} T^{*} X\right)$ be $\pi$-invariant, $p \geqslant 0$, satisfy estimates (9.4), and such that $\operatorname{supp} p \cap \operatorname{supp} e=\emptyset$ and $\operatorname{supp}(1-p) \cap K=\emptyset$. (Here $p$ can be regarded as a function on ${ }^{\mathrm{sc}} \dot{T}^{*} X$.) Let $\psi_{0} \in \mathcal{C}_{c}^{\infty}(\mathbb{R} ;[0,1])$ be identically 1 near $[\lambda-\delta, \lambda+\delta]$, and let $P \in \Psi_{\mathrm{Sc}}^{-\infty, 0}(X, \mathcal{C})$ be such that $\hat{P}_{a, 0}(\zeta)=p(\zeta) \psi_{0}\left(\hat{H}_{a}(\zeta)\right)$ and $\mathrm{WF}_{\mathrm{Sc}}^{\prime}\left(\psi_{0}(H)-P\right) \cap K=\emptyset$. For example, $P$ can be constructed as in Lemma 9.1.

The indicial operators of

$$
i \psi(H) P^{*} x^{-1 / 2}\left[A^{*} A, H\right] x^{-1 / 2} P \psi(H)-M \psi(H) P^{*} A^{*} A P \psi(H)
$$

are

$$
\begin{aligned}
& i \psi(H) P^{*} x^{-1 / 2}\left[\widehat{A^{*} A, H}\right] x^{-1 / 2} P \psi(H)_{a, 0}(\zeta)-M\left(\psi(H) \widehat{P^{*} A^{*} A} P \psi(H)\right)_{a, 0}(\zeta) \\
& \quad=i p(\zeta)^{2} \psi(H)\left[\widehat{A^{*} A, H}\right] \psi(H)_{a, 1}(\zeta)-M q(\zeta)^{2} p(\zeta)^{2} \psi\left(\hat{H}_{a, 0}(\zeta)\right)
\end{aligned}
$$

since $\psi_{0} \psi=\psi$. Thus, by Proposition 9.6 and as $M q \leqslant M C b^{2}$, we have

$$
\begin{aligned}
& i \psi(H) P^{*} x^{-1 / 2}\left[\widehat{A^{*} A, H}\right] x^{-1 / 2} P \psi(H)_{a, 0}(\zeta)-M\left(\psi(H) \widehat{P^{*} A^{*} A} P \psi(H)\right)_{a, 0}(\zeta) \\
& \quad \geqslant\left(2-\varepsilon^{\prime}-M C\right) b^{2} q \psi\left(\hat{H}_{a, 0}(\zeta)\right)^{2}
\end{aligned}
$$


Thus, taking into account (9.30) and the remark following Lemma 9.4, Proposition 8.3 gives

$$
\begin{aligned}
& i \psi(H) P^{*} x^{-1 / 2}\left[A^{*} A, H\right] x^{-1 / 2} P \psi(H)-M \psi(H) P^{*} A^{*} A P \psi(H) \\
& \quad \geqslant\left(2-\varepsilon^{\prime}\right) B^{*} B+F,
\end{aligned}
$$

with $B \in \Psi_{\mathrm{Sc}}^{-\infty, 0}(X, \mathcal{C}), F \in \Psi_{\mathrm{Sc}}^{-\infty, 1}(X, \mathcal{C})$,

$$
\hat{B}_{a, 0}(\zeta)=p(\zeta) b(\zeta) q(\zeta)^{1 / 2}
$$

so the second statement of (9.31) holds. Moreover, writing $\psi(H)=P \psi(H)+$ $\left(\psi_{0}(H)-P\right) \psi(H)$, and expanding the left hand side of (9.32), every term but the one given in (9.35) has operator wave front set disjoint from $K$. Letting $E$ be the sum of these terms proves the corollary.

\section{Propagation of singularities}

In this section we prove that singularities of generalized eigenfunctions of the many-body operator $H$ propagate along generalized broken bicharacteristics under the assumption that no (proper) subsystems of $H$ have a bound state. That is, due to our definition in Section 6, we assume that

$$
\hat{H}_{b, 0}(\xi) \text { has no } L^{2} \text { eigenvalues for any } b \neq 0 \quad \text { and } \quad \xi \in{ }^{\mathrm{sc}} T^{*}\left(\tilde{C}_{b} ; X\right) \text {. }
$$

The technical reason for this assumption lies in the argument of Proposition 9.6 in which a symbolic estimate is used to deduce positivity estimates for the indicial operators. However, it is clear that the generalized broken bicharacteristics of $\Delta-\lambda$ cannot be expected to describe propagation if the subsystems have bound states since in this situation even the characteristic set of $H$ (i.e. the set where $\hat{H}_{b, 0}(\zeta)$ is not invertible) changes.

Suppose that $p \in C_{a}^{\prime}=C^{\prime}$ (the regular part of $C$ ). As in Section 6, let $(x, y, z)=\left(x_{a}, y_{a}, z_{a}\right)$ be coordinates on $X$ near $p$ with $x$ defining $\partial X$ as usual, $C$ defined by $x=0, y=0$, chosen so that every $C_{b}$ with $p \in C_{b}$ (which implies $C_{a} \subset C_{b}$ ) is a product-linear submanifold of $\partial X$ in these local coordinates, i.e. it is of the form $\left\{(y, z): A_{b} y=0\right\}$ where $A=A_{b}$ is a matrix. In addition, as in Section 6, we arrange that at $C, \partial_{y_{j}}=\partial_{\left(y_{a}\right)_{j}}$ is perpendicular to $T C$ for each $j$ (with respect to $h$ ) and they are orthonormal with respect to each other at $p$. Let $(\tau, \mu, \nu)=\left(\tau_{a}, \mu_{a}, z_{a}\right)$ denote the sc-dual variables, so we write elements of ${ }^{\mathrm{sc}} T^{*} X$ as

$$
\tau \frac{d x}{x^{2}}+\mu \cdot \frac{d y}{x}+\nu \cdot \frac{d z}{x}
$$

Thus, at $p$ (i.e. on ${ }^{\mathrm{sc}} T_{p}^{*} X$ ) the metric function of $h$ is of the form $|\mu|^{2}+\tilde{h}(z, \nu)$ with $|\mu|$ denoting the Euclidean length of $\mu$ and $\tilde{h}$ is the metric function of the restriction of $h$ to $T C$. When talking about $C_{b}$, we sometimes write the corresponding orthogonal splitting of $y$ as $y=\left(y^{\prime}, y^{\prime \prime}\right)$, so $C_{b}$ is defined by $A_{b} y=y^{\prime}=0$ in $\partial X$.

Recall that $\pi_{0 a}:{ }^{\mathrm{sc}} T_{C}^{*} X \rightarrow{ }^{\mathrm{sc}} T^{*}(C ; X)$ is the (orthogonal) projection given by the metric at $C$. Thus, in our local coordinates $(y, z, \tau, \mu, \nu)$ on ${ }^{\mathrm{sc}} T_{\partial X}^{*} X, \pi_{0 a}(0, z, \tau, \mu, \nu)=(z, \tau, \nu)$. We use composition with the projection ${ }^{\mathrm{sc}} T_{\partial X}^{*} X$ to ${ }^{\mathrm{sc}} T_{C}^{*} X$ given by our choice of local coordinates, $(y, z, \tau, \mu, \nu) \mapsto(z, \tau, \mu, \nu)$, to extend $\pi_{0 a}$ to a map, denoted by $\pi_{0 a}^{e}$, from ${ }^{\mathrm{sc}} T_{\partial X}^{*} X$ to ${ }^{\mathrm{sc}} T^{*}(C ; X)$. Thus, $\pi_{0 a}^{e}(y, z, \tau, \mu, \nu)=(z, \tau, \nu)$. 
The propagation of singularities estimate in directions tangential to $C$ proceeds much as in the 3-body case. In fact, essentially the same operator as there gives a positive commutator, see Propositions 10.6-10.7; the functional analysis part of the argument is much as in the normal case which we proceed to examine. Recall that the normal part of the characteristic set of $H-\lambda$ over $C^{\prime}$ is

$$
\Sigma_{n}(\lambda) \cap{ }^{\mathrm{sc}} T_{C^{\prime}}^{*}(C ; X)=\left\{(z, \tau, \nu): \tau^{2}+\tilde{h}(z, \nu)<\lambda\right\} .
$$

Since the characteristic set $\Sigma_{\Delta-\lambda}$ of $\Delta-\lambda$ is given by $\tau^{2}+|\nu|_{z}^{2}+|\mu|^{2}=\lambda$ at $p$, the condition $\pi(\tilde{\xi}) \in \Sigma_{n}(\lambda) \cap{ }^{\mathrm{sc}} T_{p}^{*}(C ; X), \tilde{\xi} \in \Sigma_{\Delta-\lambda}$ implies that $\mu \neq 0$. Since the rescaled Hamilton vector field ${ }^{\text {sc }} H_{g}$ of $\Delta$ (restricted to ${ }^{\mathrm{sc}} T_{\partial X}^{*} X$ ) is given by

$$
{ }^{\mathrm{sc}} H_{g}=2 \tau\left(\mu \cdot \partial_{\mu}+\nu \cdot \partial_{\nu}\right)-2 h \partial_{\tau}+H_{h}
$$

the $\partial_{y}$ component of ${ }^{\mathrm{sc}} H_{g}$ at $p$ is $2 \mu \cdot \partial_{y}$, meaning that bicharacteristics of $\Delta$ through $\tilde{\xi}$ are normal to ${ }^{\mathrm{sc}} T_{C}^{*} X$. In addition, with $\eta=y \cdot \mu, \eta$ is $\pi$-invariant and can be used to parameterize bicharacteristic curves near $\xi=\pi(\tilde{\xi})$. In fact, at each $C_{b}$ with $p \in C_{b}, \eta=\mu \cdot y$ has the property that if we split $y=\left(y^{\prime}, y^{\prime \prime}\right)$ so that $x=0, y^{\prime}=0$ defines $C_{b}$ then $\mu \cdot y=\mu^{\prime} \cdot y^{\prime}+\mu^{\prime \prime} \cdot y^{\prime \prime}$ is independent of $\mu^{\prime}$ at $y^{\prime}=0$, so $\eta$ is $\pi$-invariant. Moreover, ${ }^{\text {sc }} H_{g} \eta(\tilde{\xi})=2|\mu|^{2}>0$, so $\eta$ can be used to parameterize the generalized broken bicharacteristics near $\xi$ as claimed. We remark that $\tau$ is another possible variable to use for the parameterization, as usual.

We now proceed to prove two normal propagation estimates. The first one will be less precise, but it works under our most general assumptions. On the other hand, the second estimate requires that all elements of $\mathcal{C}$ be totally geodesic, but it locates the incoming singularities more precisely. Although the consequences are the same, as far as propagation along generalized broken bicharacteristics is concerned (due to the geometry of these bicharacteristics), the finer estimate is worth proving since it is closer to the tangential estimates in spirit and it applies in the setting of most interest, Euclidean many-body scattering.

We only state the following propagation result for propagation in the forward direction along the generalized broken bicharacteristics. A similar result holds in the backward direction, i.e. if we replace $\eta(\xi)<0$ by $\eta(\xi)>0$ in (10.5); the proof in this case only requires changes in some signs in the argument given below.

Proposition 10.1. - Suppose that $H$ is a many-body Hamiltonian satisfying (10.1). Let $u \in \mathcal{C}^{-\infty}(X), \lambda>0$. Let $\xi_{0}=\left(z_{0}, \tau_{0}, \nu_{0}\right) \in \Sigma_{n}(\lambda) \cap{ }^{\mathrm{sc}} T_{C^{\prime}}^{*}(C ; X)$ and let $\eta=y \cdot \mu$ be the $\pi$-invariant function defined in the local coordinates discussed above. If there exists a neighborhood $U$ of $\xi_{0}$ in $\dot{\Sigma}$ such that

$$
\xi \in U \quad \text { and } \quad \eta(\xi)<0 \Rightarrow \xi \notin \mathrm{WF}_{\mathrm{Sc}}(u)
$$

then $\xi_{0} \notin \mathrm{WF}_{\mathrm{Sc}}(u)$.

Remark 10.2. - Note that $\eta(\xi)<0$ implies $y \neq 0$, so $\xi \notin{ }^{\mathrm{sc}} T_{C^{\prime}}^{*}(C ; X)$.

Remark 10.3. - We recall from Section 5 that every neighborhood $U$ of $\xi_{0}=\left(z_{0}, \tau_{0}, \nu_{0}\right) \in$ $\Sigma_{n}(\lambda) \cap{ }^{\mathrm{sc}} T_{C^{\prime}}^{*}(C ; X)$ contains an open set of the form

$$
\left\{\xi:|y(\xi)|^{2}+\left|z(\xi)-z_{0}\right|^{2}+\left|\tau(\xi)-\tau_{0}\right|^{2}+\left|\nu(\xi)-\nu_{0}\right|^{2}<\delta\right\}
$$

Note also that (10.5) implies the same statement with $U$ replaced by any smaller neighborhood of $\xi_{0}$; in particular, for the set (10.6). 
Proof. - The main step in the proof is the construction of an operator which has a microlocally positive commutator with $H$ near $\xi_{0}$. In fact, we construct the symbol of this operator. This symbol will not be a scattering symbol, i.e. it will not be in $\mathcal{C}^{\infty}\left(\mathbb{S}_{+}^{n} \times \mathbb{S}_{+}^{n}\right)$, only due to its behavior as $\mu \rightarrow \infty$ corresponding to its $\pi$-invariance. This will be accommodated by composing its quantization with a cutoff in the spectrum of $H, \phi(H), \phi \in \mathcal{C}_{c}^{\infty}(\mathbb{R})$ supported near $\lambda$, as discussed in Lemma 9.1. This approach simply extends the one taken in the three-body scattering proof of [40], though the actual construction is different due to the more complicated geometry.

Employing an iterative argument as usual, we may assume that $\xi_{0} \notin \mathrm{WF}_{\mathrm{Sc}}^{*, l}(u)$ and we need to show that $\xi_{0} \notin \mathrm{WF}_{\mathrm{Sc}}^{*, l+1 / 2}(u)$.

First we define a distance function to $\xi_{0}$. Thus, we let

$$
\omega=|y|^{2}+\left|z-z_{0}\right|^{2}+\left|\tau-\tau_{0}\right|^{2}+\left|\nu-\nu_{0}\right|^{2}
$$

|.| denoting the Euclidean norm. Then $\omega$ vanishes quadratically at $\xi_{0}$, so $|d \omega| \leqslant C_{1}^{\prime} \omega^{1 / 2}$. In particular,

$$
\left|{ }^{\mathrm{sc}} H_{g} \omega\right| \leqslant C_{1} \omega^{1 / 2} .
$$

Next, we use the variable $\eta=y \cdot \mu$ to measure propagation. Let

$$
c_{0}=\lambda-\tau_{0}^{2}-\left|\nu_{0}\right|_{z_{0}}^{2}>0 .
$$

Since the $\partial_{y}$ component of ${ }^{\mathrm{sc}} H_{g}$ at $\left(0, z_{0}, \tau, \mu, \nu\right)$ is $2 \mu$, we see that

$$
{ }^{\mathrm{sc}} H_{g} \eta-2|\mu|^{2} \mid \leqslant C_{2}^{\prime}\left(|y|+\left|z-z_{0}\right|\right) \leqslant C_{2} \omega^{1 / 2} .
$$

In addition,

$$
\begin{aligned}
\left.\left|\lambda-\tau_{0}^{2}-\right| \nu_{0}\right|_{z_{0}} ^{2}-|\mu|^{2} \mid & \leqslant|\lambda-g|+\left.\left|g-\tau_{0}^{2}-\right| \nu_{0}\right|_{z_{0}} ^{2}-|\mu|^{2} \mid \\
& \leqslant|\lambda-g|+C^{\prime}\left(|y|+\left|z-z_{0}\right|+\left|\tau-\tau_{0}\right|+\left|\nu-\nu_{0}\right|\right) \\
& \leqslant|\lambda-g|+C_{3} \omega^{1 / 2}
\end{aligned}
$$

so we conclude that

$$
\left|{ }^{\mathrm{sc}} H_{g} \eta-2 c_{0}\right| \leqslant C_{4}\left(|\lambda-g|+\omega^{1 / 2}\right) .
$$

For $\beta>0, \delta>0$, with other restrictions to be imposed later on, let

$$
\phi=\eta+\frac{\beta}{\delta} \omega
$$

so $\phi$ is a $\pi$-invariant function. Let $\chi_{0} \in \mathcal{C}^{\infty}(\mathbb{R})$ be equal to 0 on $(-\infty, 0]$ and $\chi_{0}(t)=\exp (-1 / t)$ for $t>0$. Thus, $\chi_{0}^{\prime}(t)=t^{-2} \chi_{0}(t)$. Let $\chi_{1} \in \mathcal{C}^{\infty}(\mathbb{R})$ be 0 on $(-\infty, 0], 1$ on $[1, \infty)$, with $\chi_{1}^{\prime} \geqslant 0$ satisfying $\chi_{1}^{\prime} \in \mathcal{C}_{c}^{\infty}((0,1))$. Furthermore, for $A_{0}>0$ large, to be determined, let

$$
q=\chi_{0}\left(A_{0}^{-1}(2-\phi / \delta)\right) \chi_{1}(y \cdot \mu / \delta+2) .
$$

Thus, on $\operatorname{supp} q$ we have $\phi \leqslant 2 \delta$ and $y \cdot \mu \geqslant-2 \delta$. Since $\omega \geqslant 0$, the first of these inequalities implies that $y \cdot \mu \leqslant 2 \delta$, so on $\operatorname{supp} q$

$$
|y \cdot \mu| \leqslant 2 \delta
$$


Hence,

$$
\omega \leqslant(\delta / \beta)(2 \delta-y \cdot \mu) \leqslant 4 \delta^{2} \beta^{-1} .
$$

The role that $A_{0}$ large plays is that it increases the size of the first derivatives of $q$ relative to the size of $q$, hence it will allow us to give a bound for $q$ in terms of a small multiple of its derivative along the Hamilton vector field; see (10.26)-(10.27).

We now proceed to estimate ${ }^{\mathrm{sc}} H_{g} \phi$. First, by (10.12) and (10.8),

$$
\left|{ }^{\mathrm{sc}} H_{g} \phi-2 c_{0}\right|<C_{4}\left(|\lambda-g|+\omega^{1 / 2}\right)+\frac{C_{1} \beta}{\delta} \omega^{1 / 2} .
$$

So let

$$
\beta=\frac{c_{0}^{2}}{\left(8 C_{1}\right)^{2}} \quad \text { and } \quad \delta_{0}=\frac{c_{0} \sqrt{\beta}}{8 C_{4}} .
$$

Under the additional assumptions

$$
\delta<\delta_{0} \quad \text { and } \quad|\lambda-g|<\frac{c_{0}}{4 C_{4}}
$$

we have $\omega^{1 / 2} \leqslant c_{0} /\left(4 C_{4}\right)$, so we conclude that $\left|{ }^{\text {sc }} H_{g} \phi-2 c_{0}\right| \leqslant c_{0}$, hence

$$
{ }^{\mathrm{sc}} H_{g} \phi \geqslant c_{0}>0 \text {. }
$$

This at once gives a positivity estimate for ${ }^{\mathrm{sc}} H_{g} q$ near $\xi_{0}$. Namely,

$$
\begin{aligned}
{ }^{\mathrm{sc}} H_{g} q= & -A_{0}^{-1} \delta^{-1} \chi_{0}^{\prime}\left(A_{0}^{-1}(2-\phi / \delta)\right) \chi_{1}(y \cdot \mu / \delta+2)^{\mathrm{sc}} H_{g} \phi \\
& +\delta^{-1} \chi_{0}\left(A_{0}^{-1}(2-\phi / \delta)\right) \chi_{1}^{\prime}(y \cdot \mu / \delta+2)^{\mathrm{sc}} H_{g} \eta .
\end{aligned}
$$

Thus,

$$
{ }^{\mathrm{sc}} H_{g} q=-\tilde{b}^{2}+e
$$

with

$$
\tilde{b}^{2}=A_{0}^{-1} \delta^{-1} \chi_{0}^{\prime}\left(A_{0}^{-1}(2-\phi / \delta)\right) \chi_{1}(y \cdot \mu / \delta+2)^{\mathrm{sc}} H_{g} \phi .
$$

Hence, with

$$
b^{2}=c_{0} A_{0}^{-1} \delta^{-1} \chi_{0}^{\prime}\left(A_{0}^{-1}(2-\phi / \delta)\right) \chi_{1}(y \cdot \mu / \delta+2),
$$

we have

$$
{ }^{\mathrm{sc}} H_{g} q \leqslant-b^{2}+e .
$$

Moreover,

$$
b^{2} \geqslant\left(c_{0} A_{0} / 16\right) q
$$

since $\phi \geqslant y \cdot \mu \geqslant-2 \delta$ on $\operatorname{supp} q$, so

$$
\begin{aligned}
\chi_{0}^{\prime}\left(A_{0}^{-1}(2-\phi / \delta)\right) & =A_{0}^{2}(2-\phi / \delta)^{-2} \chi_{0}\left(A_{0}^{-1}(2-\phi / \delta)\right) \\
& \geqslant\left(A_{0}^{2} / 16\right) \chi_{0}\left(A_{0}^{-1}(2-\phi / \delta)\right) .
\end{aligned}
$$

On the other hand, $e$ is supported where

$$
-2 \delta \leqslant y \cdot \mu \leqslant-\delta, \quad \omega^{1 / 2} \leqslant 2 \beta^{-1 / 2} \delta,
$$


so, for $\delta>0$ sufficiently small, in the region which we know is disjoint from $\operatorname{WF}_{\mathrm{Sc}}(u)$. Moreover, on $\operatorname{supp} q$,

$$
-2 \delta \leqslant y \cdot \mu \leqslant 2 \delta, \quad \omega^{1 / 2} \leqslant 2 \beta^{-1 / 2} \delta,
$$

so, for $\delta>0$ sufficiently small, we deduce from the inductive hypothesis that $\operatorname{supp} q$ (hence $\operatorname{supp} b)$ is disjoint from $\mathrm{WF}_{\mathrm{Sc}}^{*, l+1 / 2}(u)$. In addition, by choosing $\delta>0$ sufficiently small, we can assume that the support of $q, e$ and $b$ are all disjoint from $\mathrm{WF}_{\mathrm{Sc}}((H-\lambda) u)$.

Moreover, with $\partial$ denoting a partial derivative with respect to one of $(y, z, \tau, \mu, \nu)$,

$$
\begin{aligned}
\partial q= & -A_{0}^{-1} \delta^{-1} \chi_{0}^{\prime}\left(A_{0}^{-1}(2-\phi / \delta)\right) \chi_{1}(\eta / \delta+2) \partial \phi \\
& -\delta^{-1} \chi_{0}\left(A_{0}^{-1}(2-\phi / \delta)\right) \chi_{1}^{\prime}(\eta / \delta+2) \partial \eta .
\end{aligned}
$$

As $y=0$ is outside the support of the second term, and as $\partial_{\mu} \phi$ vanishes at $y=0$, we conclude that for any multiindex $\beta$,

$$
\left|\partial_{\mu}^{\beta} d q\right| \leqslant C_{\beta} b^{2} \quad \text { at } y=0 .
$$

More generally, at any $C_{b}$ with $p \in C_{b}$, defined by $x=0, y^{\prime}=0$, as above, $\phi$ is independent of $\mu^{\prime}$ at $y^{\prime}=0$ so outside suppe

$$
\left|\partial_{\mu^{\prime}}^{\beta} d q\right| \leqslant C_{\beta} b^{2} \quad \text { at } y^{\prime}=0 .
$$

In fact, outside $\operatorname{supp} e$, but in the set where $b$ is positive,

$$
b^{-2} \partial q=c_{0}^{-1} \partial \phi
$$

so the uniform bounds of (9.30) also follow.

Let $\tilde{\psi} \in \mathcal{C}_{c}^{\infty}(\mathbb{R})$ be identically 1 near 0 and supported sufficiently close to 0 so that the product decomposition of $X$ near $\partial X$ is valid on $\operatorname{supp} \tilde{\psi}$. We also define

$$
\tilde{q}=\tilde{\psi}(x) q
$$

Thus, $\tilde{q} \in \mathcal{C}^{\infty}\left({ }^{\mathrm{sc}} T^{*} X\right)$ is a $\pi$-invariant function satisfying (9.4). Let $A$ be the operator given by Lemma 9.1 with $\tilde{q}$ in place of $q$, so in particular its indicial operators are $q(\zeta) \psi_{0}\left(\hat{H}_{b, 0}(\zeta)\right)$. Note that (9.16) holds with $C=16 c_{0}^{-1} A_{0}^{-1}$ by (10.26). So suppose that $M>0$ and $\varepsilon^{\prime}>0$. Choose $A_{0}$ so large that $M C<\varepsilon^{\prime}$. By Corollary 9.7 and the hypothesis (10.1), we deduce the following statement. For any $K^{\prime} \subset{ }^{\mathrm{sc}} \dot{T}^{*} X$ compact with supp $e \cap K^{\prime}=\emptyset$ there exist $\delta^{\prime}>0$, $B, E \in \Psi_{\mathrm{Sc}}^{-\infty, 0}(X, \mathcal{C}), F \in \Psi_{\mathrm{Sc}}^{-\infty, 1}(X, \mathcal{C})$ with

$$
\begin{aligned}
& \mathrm{WF}_{\mathrm{Sc}}^{\prime}(E) \cap K^{\prime}=\emptyset, \quad \mathrm{WF}_{\mathrm{Sc}}^{\prime}(F) \subset \operatorname{supp} \tilde{q}, \\
& \hat{B}_{a, 0}(\zeta)=b(\zeta) q(\zeta)^{1 / 2} \psi\left(\hat{H}_{a, 0}(\zeta)\right), \quad \zeta \in K^{\prime},
\end{aligned}
$$

such that if $\psi \in \mathcal{C}_{c}^{\infty}(\mathbb{R})$ is supported in $\left(\lambda-\delta^{\prime}, \lambda+\delta^{\prime}\right)$ then

$$
\begin{aligned}
& i \psi(H) x^{-1 / 2}\left[A^{*} A, H\right] x^{-1 / 2} \psi(H)-M \psi(H) A^{*} A \psi(H) \\
& \quad \geqslant\left(2-2 \varepsilon^{\prime}\right) B^{*} B+E+F .
\end{aligned}
$$

Let

$$
\Lambda_{r}=x^{-l-1 / 2}(1+r / x)^{-1}, \quad r \in(0,1),
$$


so $\Lambda_{r} \in \Psi_{\mathrm{Sc}}^{0,-l+1 / 2}(X, \mathcal{C})$ for $r \in(0,1)$ and it is uniformly bounded in $\Psi_{\mathrm{Scc}}^{0,-l-1 / 2}(X, \mathcal{C})$. The last statement follows from $(1+r / x)^{-1}$ being uniformly bounded as a 0 th order symbol, i.e. from $\left(x \partial_{x}\right)^{k}(1+r / x)^{-1} \leqslant C_{k}$ uniformly $\left(C_{k}\right.$ independent of $\left.r\right)$. In particular, note that

$$
\begin{aligned}
x \partial_{x}(1+r / x)^{-1} & =r x^{-1}(1+r / x)^{-2} \\
& =\frac{r}{x+r}(1+r / x)^{-1}=\left(1-(1+r / x)^{-1}\right)(1+r / x)^{-1} .
\end{aligned}
$$

Since $0 \leqslant \frac{r}{x+r} \leqslant 1,\left(x \partial_{x}\right)(1+r / x)^{-1}$ is a uniformly bounded multiple of $(1+r / x)^{-1}$, and in fact this bounded multiplier is uniformly bounded as a 0th order symbol. This also implies that

$$
\left[(1+r / x)^{-1}, H\right](1+r / x)
$$

which is a priori uniformly bounded in $\Psi_{\mathrm{Scc}}^{1,0}(X, \mathcal{C})$ only, is in fact uniformly bounded in $\Psi_{\text {Scc }}^{1,1}(X, \mathcal{C})$.

We also define

$$
A_{r}=A \Lambda_{r} x^{-1 / 2} \psi(H), \quad B_{r}=B \Lambda_{r}, \quad E_{r}=\Lambda_{r} E \Lambda_{r} .
$$

Then, with $\psi_{0} \in \mathcal{C}_{c}^{\infty}(\mathbb{R} ;[0,1])$ identically 1 near $\operatorname{supp} \psi$,

$$
\begin{aligned}
i x^{l+1 / 2}\left[A_{r}^{*} A_{r}, H\right] x^{l+1 / 2}= & i(1+r / x)^{-1} \psi(H) x^{-1 / 2}\left[A^{*} A, H\right] x^{-1 / 2} \psi(H)(1+r / x)^{-1} \\
& +i(1+r / x)^{-1} \psi(H) A^{*}(1+r / x) x^{l+1 / 2}\left[\Lambda_{r} x^{-1 / 2}, H\right] \\
& \times x^{-1 / 2} \psi_{0}(H) A \psi(H)(1+r / x)^{-1} \\
& +i(1+r / x)^{-1} \psi(H) A^{*} \psi_{0}(H) x^{-1 / 2}\left[\Lambda_{r} x^{-1 / 2}, H\right] \\
& \times(1+r / x) x^{l+1 / 2} A \psi(H)(1+r / x)^{-1}+H_{r},
\end{aligned}
$$

where $H_{r}$ is uniformly bounded in $\Psi_{\mathrm{Scc}}^{-\infty, 1}(X, \mathcal{C})$. Note that $H_{r}$ arises by commuting $A$, powers of $x$ and $\Lambda_{r}$ through other operators, but as the indicial operators of $A$ and $x$ are multiples of the identity, $A, x$ and $\Lambda_{r}$ commute with these operators to top order, and in case of $\Lambda_{r}$, the commutator is uniformly bounded as an operator of one lower order (than the order of the product of $\Lambda_{r}$ with such operators). Then, multiplying (10.36) by $(1+r / x)^{-1}$ from the left and right and rearranging the terms we obtain the following estimate of bounded self-adjoint operators on $L_{\mathrm{sc}}^{2}(X)$ :

$$
\begin{aligned}
i x^{l+1 / 2}\left[A_{r}^{*} A_{r}, H\right] x^{l+1 / 2}-(1+r / x)^{-1}\left(\psi(H) A^{*}\left(G_{r}^{*}+G_{r}\right) A \psi(H)\right. \\
\left.\quad+M \psi(H) A^{*} A \psi(H)\right)(1+r / x)^{-1} \\
\quad+x^{l+1 / 2}\left(\left(2-\varepsilon^{\prime}\right) B_{r}^{*} B_{r}+E_{r}+F_{r}\right) x^{l+1 / 2}
\end{aligned}
$$

where

$$
G_{r}=i \psi_{0}(H) x^{-1 / 2}\left[\Lambda_{r} x^{-1 / 2}, H\right](1+r / x) x^{l+1 / 2},
$$

and $F_{r} \in \Psi_{\mathrm{Sc}}^{-\infty,-2 l+1}(X, \mathcal{C})$ is uniformly bounded in $\Psi_{\mathrm{Scc}}^{-\infty,-2 l}(X, \mathcal{C})$ as $r \rightarrow 0$. Since $\left[(1+r / x)^{-1}, H\right](1+r / x)$ is uniformly bounded in $\Psi_{\mathrm{Scc}}^{1,1}(X, \mathcal{C})$, we conclude that $G_{r}$ is uniformly bounded in $\Psi_{\mathrm{Scc}}^{-\infty, 0}(X, \mathcal{C})$, hence as a bounded operator on $L_{\mathrm{sc}}^{2}(X)$. Thus, if $M>0$ is chosen sufficiently large, then $G_{r}+G_{r}^{*} \geqslant-M$ for all $r \in(0,1)$, so

$$
(1+r / x)^{-1} \psi(H) A^{*}\left(G_{r}+G_{r}^{*}+M\right) A \psi(H)(1+r / x)^{-1} \geqslant 0 .
$$


Adding this to (10.42) shows that

$$
i x^{l+1 / 2}\left[A_{r}^{*} A_{r}, H\right] x^{l+1 / 2} \geqslant x^{l+1 / 2}\left(\left(2-\varepsilon^{\prime}\right) B_{r}^{*} B_{r}+E_{r}+F_{r}\right) x^{l+1 / 2} .
$$

The point of the commutator calculation is that in $L_{\mathrm{sc}}^{2}(X)$

$$
\begin{aligned}
\left\langle u,\left[A_{r}^{*} A_{r}, H\right] u\right\rangle & =\left\langle u, A_{r}^{*} A_{r}(H-\lambda) u\right\rangle-\left\langle u,(H-\lambda) A_{r}^{*} A_{r} u\right\rangle \\
& =2 i \operatorname{Im}\left\langle u, A_{r}^{*} A_{r}(H-\lambda) u\right\rangle ;
\end{aligned}
$$

the pairing makes sense for $r>0$ since $A_{r} \in \Psi_{\mathrm{Sc}}^{-\infty,-l}(X, \mathcal{C})$. Now apply (10.42) to $x^{-l-1 / 2} u$ and pair it with $x^{-l-1 / 2} u$ in $L_{\mathrm{sc}}^{2}(X)$. Then for $r>0$

$$
\left\|B_{r} u\right\|^{2} \leqslant\left|\left\langle u, E_{r} u\right\rangle\right|+\left|\left\langle u, F_{r} u\right\rangle\right|+2\left|\left\langle u, A_{r}^{*} A_{r}(H-\lambda) u\right\rangle\right| .
$$

Letting $r \rightarrow 0$ now keeps the right hand side of (10.47) bounded. In fact, $A_{r}(H-\lambda) u$ $\in \dot{\mathcal{C}}^{\infty}(X)$ remains bounded in $\dot{\mathcal{C}}^{\infty}(X)$ as $r \rightarrow 0$. Similarly, by (10.35), $E_{r} u$ remains bounded in $\dot{\mathcal{C}}^{\infty}(X)$ as $r \rightarrow 0$ if we chose $K^{\prime}$ so large that $\mathrm{WF}_{\mathrm{Sc}}(u) \subset K^{\prime}$. Also, $F_{r}$ is bounded in $\mathcal{B}\left(H_{\mathrm{sc}}^{m, l}(X), H_{\mathrm{sc}}^{-m,-l}(X)\right)$, so $\left\langle u, F_{r} u\right\rangle$ stays bounded by $(10.35)$ as well. These estimates show that $B_{r} u$ is uniformly bounded in $L_{\mathrm{sc}}^{2}(X)$. Since $(1+r / x)^{-1} \rightarrow \operatorname{Id}$ strongly on $\mathcal{B}\left(H_{\mathrm{sc}}^{m^{\prime}, l^{\prime}}(X), H_{\mathrm{sc}}^{m^{\prime}, l^{\prime}}(X)\right)$, we conclude that $B x^{-l-1 / 2} u \in L_{\mathrm{sc}}^{2}(X)$. By (10.35) and Proposition 5.5 this implies that for every $m$,

$$
\xi_{0} \notin \mathrm{WF}_{\mathrm{Sc}}^{m, l+1 / 2}(u) .
$$

This is exactly the iterative step we wanted to prove. In the next step we decrease $\delta>0$ slightly to ensure that $\mathrm{WF}_{\mathrm{Sc}}^{\prime}(F) \subset \operatorname{supp} \tilde{q}$ is disjoint from $\mathrm{WF}_{\mathrm{Sc}}^{m, l+1 / 2}(u)$.

To state and prove the finer estimate under the assumption that all elements of $\mathcal{C}$ are totally geodesic, first note that in geodesic normal coordinates around $p \in C^{\prime}, h-\left(|\mu|^{2}+|\nu|^{2}\right)$ vanishes together with its first derivatives at $p=(0,0)$. Thus, ${ }^{\text {sc }} H_{g}$ agrees with $W^{b}$ on ${ }^{\text {sc }} T_{p}^{*} X$ where

$$
W^{\mathrm{b}}=2 \tau\left(\mu \cdot \partial_{\mu}+\nu \cdot \partial_{\nu}\right)-2\left(|\mu|^{2}+|\nu|^{2}\right) \partial_{\tau}+2 \nu \cdot \partial_{z}+2 \mu \cdot \partial_{y}
$$

We will use $W^{b}$ to model the bicharacteristic flow of $H$. Note that $W^{b}$ is the (rescaled) Hamilton vector field of the metric function $\tau^{2}+|\nu|^{2}+|\mu|^{2}$, i.e. where we replace the actual metric $h$ by a flat one.

We remark that it is the $\partial_{\mu}$ and $\partial_{\nu}$ components of ${ }^{\mathrm{sc}} H_{g}$ that differ from $W^{\mathrm{b}}$ on ${ }^{\mathrm{sc}} T_{p}^{*} X$ if we do not assume that the elements of $\mathcal{C}$ are totally geodesic. The former is inconsequential since we only consider $\pi$-invariant functions (in particular, the only $\mu$-dependence is via $\eta=y \cdot \mu$ ), but the latter rules out the more precise location of the singularities given in the following proposition.

Proposition 10.4. - Suppose that $H$ is a many-body Hamiltonian satisfying (10.1) and that every element of $\mathcal{C}$ is totally geodesic with respect to $h$. Let $u \in \mathcal{C}^{-\infty}(X), \lambda>0$. Given $K \subset \Sigma_{n}(\lambda) \cap{ }^{\mathrm{sc}} T_{C^{\prime}}^{*}(C ; X)$ compact with $K \cap \mathrm{WF}_{\mathrm{Sc}}((H-\lambda) u)=\emptyset$ there exist constants $C_{0}>0, \delta_{0}>0$ such that the following holds. If $\xi_{0}=\left(0, \tau_{0}, \nu_{0}\right) \in K$ and for some $0<\delta<\delta_{0}$, $C_{0} \delta^{1 / 2} \leqslant \varepsilon<1$ and for all $\alpha=(y, z, \tau, \mu, \nu) \in{ }^{\mathrm{sc}} T_{\partial X}^{*} X \cap \Sigma_{\Delta-\lambda}$

$$
\begin{aligned}
\alpha & \in{ }^{\mathrm{sc}} T_{C_{b}^{\prime}}^{*} X \quad \text { and }\left|\pi_{0 a}^{e}\left(\exp \left(\delta W^{\mathrm{b}}\right)(\alpha)\right)-\xi_{0}\right| \leqslant \varepsilon \delta \quad \text { and }\left|y\left(\exp \left(\delta W^{\mathrm{b}}\right)(\alpha)\right)\right| \leqslant \varepsilon \delta \\
& \Rightarrow \pi_{0 b}(\alpha) \notin \mathrm{WF}_{\mathrm{Sc}}(u)
\end{aligned}
$$

then $\xi_{0} \notin \mathrm{WF}_{\mathrm{Sc}}(u)$. 
Remark 10.5. - The estimate is stated in the form (10.50) for simplicity of statement. The proof provides a slightly different estimate, involving the backward flow $\exp \left(-\delta W^{b}\right)(\tilde{\xi})$, $\tilde{\xi}_{0} \in \hat{\pi}^{-1}\left(\xi_{0}\right)$; the two are equivalent up to changing $C_{0}$, see Remark 10.8 for a more thorough discussion.

Proof. - We again employ an iterative argument, so we assume that $\xi_{0} \notin \mathrm{WF}_{\mathrm{Sc}}^{*, l}(u)$ and we need to show that $\xi_{0} \notin \mathrm{WF}_{\mathrm{Sc}}^{*, l+1 / 2}(u)$.

We first construct a $\mathcal{C}^{\infty}$ function $\omega$ of $z, \tau, \nu, \eta=\mu \cdot y$ and $s=|y|^{2}$ which measures the distance of bicharacteristics of $\Delta$ in $\Sigma_{\Delta-\lambda}$ from $\pi_{a 0}^{-1}\left(\xi_{0}\right) \cap \Sigma_{\Delta-\lambda}$. Thus, $\tau^{2}+|\nu|^{2}+|\mu|^{2}-\lambda$ will be small along these bicharacteristics. We will take $\omega$ of the form

$$
\omega=\omega_{0}^{2}+\left(|y|^{2}-\frac{(y \cdot \mu)^{2}}{\lambda-\tau_{0}^{2}-\left|\nu_{0}\right|^{2}}\right)^{2},
$$

where $\omega_{0}$ only depends on $z, \tau, \nu$ and $\eta=y \cdot \mu$. Note that

$$
|y|^{2}-\frac{(y \cdot \mu)^{2}}{|\mu|^{2}}=\left|y-\frac{y \cdot \mu}{|\mu|^{2}} \mu\right|^{2}
$$

is the squared distance of the integral curves of $H_{|\mu|^{2}}$, which are just straight lines, from $y=0$, so near $\Sigma_{\Delta-\lambda}$ the second term in $\omega$ gives the fourth power of this distance.

Pushing forward $W^{b}$ by the map $F:(y, z, \tau, \mu, \nu) \mapsto(z, \tau, \nu, \mu \cdot y)$ at some point $\alpha=(y, z, \tau, \mu, \nu)$, we obtain the vector

$$
\left.F_{*}\right|_{\alpha} W^{b}=2\left(\tau \eta+|\mu|^{2}\right) \partial_{\eta}+2 \tau \nu \cdot \partial_{\nu}-2\left(|\mu|^{2}+|\nu|^{2}\right) \partial_{\tau}+2 \nu \cdot \partial_{z} .
$$

Since we are interested in what happens near $\Sigma_{\Delta-\lambda} \cap{ }^{\mathrm{sc}} T_{p}^{*} X$, where $\lambda=\tau^{2}+|\nu|^{2}+|\mu|^{2}$, we are led to consider the constant coefficient vector field

$$
W_{0}=2\left(\lambda-\tau_{0}^{2}-\left|\nu_{0}\right|^{2}\right) \partial_{\eta}+2 \tau_{0} \nu_{0} \cdot \partial_{\nu}-2\left(\lambda-\tau_{0}^{2}\right) \partial_{\tau}+\nu_{0} \cdot \partial_{z}
$$

in the variables $(z, \tau, \nu, \eta)$, so

$$
\left.F_{*}\right|_{\alpha} W^{b}=W_{0}+2\left(\lambda-\tau_{0}^{2}-\left|\mu_{0}\right|^{2}-\left|\nu_{0}\right|^{2}\right)\left(-\partial_{\eta}+\partial_{\tau}\right) .
$$

Note that the $\partial_{\eta}$ component of $W_{0}$ is nonzero. Let

$$
z_{0}(t)=\frac{W_{0} z}{W_{0} \eta} t, \quad \tau_{0}(t)=\tau_{0}+\frac{W_{0} \tau}{W_{0} \eta} t, \quad \nu_{0}(t)=\nu_{0}+\frac{W_{0} \nu}{W_{0} \eta} t
$$

so

$$
\gamma: t \mapsto\left(z_{0}\left(\left(W_{0} \eta\right) t\right), \tau_{0}\left(\left(W_{0} \eta\right) t\right), \nu_{0}\left(\left(W_{0} \eta\right) t\right),\left(W_{0} \eta\right) t\right)
$$

gives a curve through $\left(\xi_{0}, 0\right)$ with tangent vector $W_{0}$. Now we define $\omega_{0}$ by

$$
\omega_{0}=\left(z-z_{0}(\eta)\right)^{2}+\left(\tau-\tau_{0}(\eta)\right)^{2}+\left(\nu-\nu_{0}(\eta)\right)^{2}
$$

so $\omega_{0}$ vanishes exactly quadratically along $\gamma$ and is positive elsewhere, and

$$
W_{0} \omega_{0}=0 .
$$


Note that by the triangle inequality

$$
|z|+\left|\tau-\tau_{0}\right|+\left|\nu-\nu_{0}\right|+|\eta| \leqslant C\left(\omega_{0}^{1 / 2}+|\eta|\right)
$$

for sufficiently large $C$.

Since for $\alpha \in \hat{\pi}^{-1}\left(\xi_{0}\right)$ we have $\left.F_{*}\right|_{\alpha}{ }^{\text {sc }} H_{g}=\left.F_{*}\right|_{\alpha} W^{b}=W_{0}$, we see that

$$
{ }^{\mathrm{sc}} H_{g}\left(z-z_{0}(\eta)\right)=0 \quad \text { at } \hat{\pi}^{-1}\left(\xi_{0}\right),
$$

i.e. when $y=0, z=0, \tau=\tau_{0}, \nu=\nu_{0}, g=\lambda$, so

$$
\left|{ }^{\mathrm{sc}} H_{g}\left(z-z_{0}(\eta)\right)\right| \leqslant C\left(|y|+\omega_{0}^{1 / 2}+|\lambda-g|\right) .
$$

Hence,

$$
\left|{ }^{\mathrm{sc}} H_{g}\left(z-z_{0}(\eta)\right)^{2}\right| \leqslant 2 C \omega_{0}^{1 / 2}\left(|y|+\omega_{0}^{1 / 2}+|\lambda-g|\right) .
$$

Similar conclusions hold for $\tau-\tau_{0}(\eta)$ and $\nu-\nu_{0}(\eta)$, so

$$
\left|{ }^{\mathrm{sc}} H_{g} \omega_{0}\right| \leqslant C_{1}\left(|y|+\omega_{0}^{1 / 2}+|\lambda-g|\right) \omega_{0}^{1 / 2} .
$$

Next, we calculate ${ }^{\mathrm{sc}} H_{g}\left(|y|^{2}-(y \cdot \mu)^{2} /\left(\lambda-\tau_{0}^{2}-\left|\nu_{0}\right|^{2}\right)\right)$. Since the function we are differentiating vanishes quadratically at $y=0$, the same follows for its derivatives with respect to any vector field tangent to $y=0$. Since the $\partial_{y}$ component of ${ }^{\mathrm{sc}} H_{g}$ (and of $W^{\mathrm{b}}$ ) is of the form $2 \mu \cdot \partial_{y}+\sum \beta_{j} \partial_{y_{j}}$ with $\beta_{j}$ vanishing at $y=0, z=0$ (i.e. at $p$ ), we conclude that

$$
\left|\left({ }^{\mathrm{sc}} H_{g}-2 \mu \cdot \partial_{y}\right)\left(|y|^{2}-(y \cdot \mu)^{2} /\left(\lambda-\tau_{0}^{2}-\left|\nu_{0}\right|^{2}\right)\right)\right| \leqslant C_{2}|y|(|y|+|z|) .
$$

On the other hand,

$$
\left(2 \mu \cdot \partial_{y}\right)\left(|y|^{2}-(y \cdot \mu)^{2} /\left(\lambda-\tau_{0}^{2}-\left|\nu_{0}\right|^{2}\right)\right)=4(\mu \cdot y) \frac{\lambda-\tau_{0}^{2}-\left|\nu_{0}\right|^{2}-|\mu|^{2}}{\lambda-\tau_{0}^{2}-\left|\nu_{0}\right|^{2}}
$$

But, as in (10.11),

$$
\begin{aligned}
\left.\left|\lambda-\tau_{0}^{2}-\right| \nu_{0}\right|^{2}-|\mu|^{2} \mid & \leqslant|\lambda-g|+C^{\prime}\left(|y|+|z|+\left|\tau-\tau_{0}\right|+\left|\nu-\nu_{0}\right|\right) \\
& \leqslant C_{3}\left(|\lambda-g|+|y|+\omega_{0}^{1 / 2}\right) .
\end{aligned}
$$

Thus,

$$
{ }^{\mid s c} H_{g}\left(|y|^{2}-(y \cdot \mu)^{2} /\left(\lambda-\tau_{0}^{2}-\left|\nu_{0}\right|^{2}\right)\right)\left|\leqslant C_{4}\right| y \mid\left(|\lambda-g|+|y|+\omega_{0}^{1 / 2}\right) .
$$

Our results thus far imply that

$$
\left|{ }^{\mathrm{sc}} H_{g} \omega\right| \leqslant C_{5} \omega^{1 / 2}\left(|y|+|\lambda-g|+\omega_{0}^{1 / 2}\right)^{2} .
$$

Now let $1>\varepsilon>0, \delta>0$, with other restrictions to be imposed on these later, and let

$$
\phi=\tau_{0}-\tau+\frac{1}{\varepsilon^{4} \delta^{3}} \omega .
$$

We use $\tau_{0}-\tau$ to measure propagation along the bicharacteristics; $\eta=y \cdot \mu$ would also work. We again let $\chi_{0} \in \mathcal{C}^{\infty}(\mathbb{R})$ be equal to 0 on $(-\infty, 0]$ and $\chi_{0}(t)=\exp (-1 / t)$ for $t>0$ and we let 
$\chi_{1} \in \mathcal{C}^{\infty}(\mathbb{R})$ be 0 on $(-\infty, 0], 1$ on $[1, \infty)$, with $\chi_{1}^{\prime} \geqslant 0$ satisfying $\chi_{1}^{\prime} \in \mathcal{C}_{c}^{\infty}((0,1))$. Furthermore, for $A_{0}>0$ large, to be determined, $t \in(0,1)$, let

$$
q_{t}=q=\chi_{0}\left(A_{0}^{-1}(1+t-\phi / \delta)\right) \chi_{1}\left(\left(\tau_{0}-\tau+\delta\right) /(\varepsilon \delta)+t\right) .
$$

We usually simply write $q$ in place of $q_{t}$. We only use $t$ to slightly shrink the support of $q$ in our inductive proof (i.e. as $l$ is increasing), instead of adjusting $\delta$ as in the proof of Proposition 10.1. Thus, on $\operatorname{supp} q$ we have $\phi \leqslant 2 \delta$ and $\tau_{0}-\tau \geqslant-2 \delta$. Since $\omega \geqslant 0$, the first of these inequalities implies that $\tau_{0}-\tau \leqslant 2 \delta$, so

$$
\left|\tau-\tau_{0}\right| \leqslant 2 \delta \text { and } \omega \leqslant \varepsilon^{4} \delta^{3}\left(2 \delta+\tau-\tau_{0}\right) \leqslant 4 \varepsilon^{4} \delta^{4} .
$$

Hence, $\omega_{0} \leqslant 2 \varepsilon^{2} \delta^{2}$, which together with $\left|\tau-\tau_{0}\right| \leqslant 2 \delta$ gives $|\eta|=|\mu \cdot y| \leqslant C_{6} \delta$ since the $\partial_{\tau}$ component of $W$ in non-zero. Since we also have

$$
\left.|| y\right|^{2}-(y \cdot \mu)^{2} /\left(\lambda-\tau_{0}^{2}-\left|\nu_{0}\right|^{2}\right) \mid \leqslant 2 \varepsilon^{2} \delta^{2},
$$

we conclude that $|y| \leqslant C_{7} \delta$. Thus, under the additional assumption

$$
|\lambda-g|<\delta
$$

we deduce that $\left|{ }^{\mathrm{sc}} H_{g} \omega\right| \leqslant C_{8} \varepsilon^{2} \delta^{4}$, so

$$
\left|{ }^{\mathrm{sc}} H_{g} \phi-2 h\right| \leqslant C_{8} \delta / \varepsilon^{2} .
$$

Hence, for $c_{0}>0, C_{0}>0$ appropriately chosen and for $\varepsilon \in(0,1), \delta>0$ satisfying $\delta / \varepsilon^{2}<C_{0}$, we have

$$
{ }^{\mathrm{sc}} H_{g} \phi>c_{0}>0 \text {. }
$$

Again, this directly gives a positivity estimate for ${ }^{\mathrm{sc}} H_{g} q$ near $\xi_{0}$. Now

$$
\begin{aligned}
{ }^{\mathrm{sc}} H_{g} q= & -A_{0}^{-1} \delta^{-1} \chi_{0}^{\prime}\left(A_{0}^{-1}(1+t-\phi / \delta)\right) \chi_{1}\left(\left(\tau_{0}-\tau+\delta\right) /(\varepsilon \delta)+t\right)^{\mathrm{sc}} H_{g} \phi \\
& -(\varepsilon \delta)^{-1} \chi_{0}\left(A_{0}^{-1}(1+t-\phi / \delta)\right) \chi_{1}^{\prime}\left(\left(\tau_{0}-\tau+\delta\right) /(\varepsilon \delta)+t\right)^{\mathrm{sc}} H_{g} \tau .
\end{aligned}
$$

Hence, with

$$
\begin{aligned}
b^{2} & =c_{0} A_{0}^{-1} \delta^{-1} \chi_{0}^{\prime}\left(A_{0}^{-1}(1+t-\phi / \delta)\right) \chi_{1}\left(\left(\tau_{0}-\tau+\delta\right) /(\varepsilon \delta)+t\right), \\
e & =-(\varepsilon \delta)^{-1} \chi_{0}\left(A_{0}^{-1}(1+t-\phi / \delta)\right) \chi_{1}^{\prime}\left(\left(\tau_{0}-\tau+\delta\right) /(\varepsilon \delta)+t\right)^{\mathrm{sc}} H_{g} \tau
\end{aligned}
$$

we have

$$
{ }^{\mathrm{sc}} H_{g} q \leqslant-b^{2}+e .
$$

In addition, similarly to (10.26)-(10.27), we see that

$$
b^{2} \geqslant\left(c_{0} A_{0} / 16\right) q
$$

Moreover, with $\partial$ denoting a partial derivative with respect to one of $(y, z, \tau, \mu, \nu)$,

$$
\begin{aligned}
\partial q= & -A_{0}^{-1} \delta^{-1} \chi_{0}^{\prime}\left(A_{0}^{-1}(1+t-\phi / \delta)\right) \chi_{1}\left(\left(\tau_{0}-\tau+\delta\right) /(\varepsilon \delta)+t\right) \partial \phi \\
& -(\varepsilon \delta)^{-1} \chi_{0}\left(A_{0}^{-1}(1+t-\phi / \delta)\right) \chi_{1}^{\prime}\left(\left(\tau_{0}-\tau+\delta\right) /(\varepsilon \delta)+t\right) \partial \tau .
\end{aligned}
$$


Thus, (10.31)-(10.33) hold, and hence the uniform bounds of (9.30) also follow. Now $e$ is supported where

$$
-\delta-t \varepsilon \delta \leqslant \tau_{0}-\tau \leqslant-\delta+(1-t) \varepsilon \delta, \quad \omega^{1 / 4} \leqslant \sqrt{2} \varepsilon \delta
$$

so near the backward direction along bicharacteristics through $\xi_{0}$, in the region which we know is disjoint from $\mathrm{WF}_{\mathrm{Sc}}(u)$. In addition, by choosing $\delta>0$ sufficiently small, we can assume that the support of $q, e$ and $b$ are all disjoint from $\mathrm{WF}_{\mathrm{Sc}}((H-\lambda) u)$.

From this point we can simply follow the proof of Proposition 10.1. Thus, we conclude that for every $m$,

$$
\xi_{0} \notin \mathrm{WF}_{\mathrm{Sc}}^{m, l+1 / 2}(u) .
$$

This is exactly the iterative step we wanted to prove. In the next step we decrease $t$ slightly to ensure that $\operatorname{supp} \tilde{q}_{t}$ is disjoint from $\mathrm{WF}_{\mathrm{Sc}}^{m, l+1 / 2}(u)$.

Before proving the general tangential propagation estimate, we first do it in the totally geodesic case $(\mathcal{C}$ totally geodesic). Proposition 7.1 shows that for sufficiently short times there is a unique generalized broken bicharacteristic through any point in $\Sigma_{t}(\lambda)$, namely the integral curve of ${ }^{\mathrm{sc}} H_{g}$. The simplicity of this description may already give a hint that it is particularly easy to prove the corresponding propagation estimate for singularities. Indeed, in the proof of the aforementioned proposition, we have essentially already constructed the pseudo-differential operator $A$ to commute through $H$ by defining the $\pi$-invariant function $\phi$ (which will play an analogous role to that of $\phi$ in the proof of normal propagation). The following argument may also clarify the close relationship between proving results about the geometry of the generalized broken bicharacteristics and the positive commutator proof of propagation estimates. Again, we only state it for forward propagation.

Proposition 10.6. - Suppose that $H$ is a many-body Hamiltonian satisfying (10.1). Suppose also that every element of $\mathcal{C}$ is totally geodesic with respect to $h$. Let $u \in \mathcal{C}^{-\infty}(X)$, $\lambda>0$. Let $\xi_{0} \in \Sigma_{t}(\lambda) \cap{ }^{\mathrm{sc}} T_{C^{\prime}}^{*}(C ; X), C=C_{a}$, satisfy $\xi_{0} \notin \mathrm{WF}_{\mathrm{Sc}}((H-\lambda) u)$. Then there exists $\varepsilon^{\prime}>0$ such that if in addition for some $s \in\left(-\varepsilon^{\prime}, 0\right)$ we have

$$
\pi_{0 a}\left(\exp \left(s^{\mathrm{sc}} H_{g}\right)\left(\hat{\pi}^{-1}\left(\xi_{0}\right)\right)\right) \notin \mathrm{WF}_{\mathrm{Sc}}(u)
$$

then $\xi_{0} \notin \mathrm{WF}_{\mathrm{Sc}}(u)$.

Proof. - First note that there is nothing to prove if $\xi_{0} \in R_{+}(\lambda) \cup R_{-}(\lambda)$, so from now on we assume that $\xi_{0} \notin R_{+}(\lambda) \cup R_{-}(\lambda)$. The proof is very similar to the previous one and the positive commutator construction is exactly the same as in three-body scattering [40, Proposition 15.4], based on the $\pi$-invariant function $\phi$ used here in the proof of Proposition 7.1. Thus, we take local coordinates centered at $C$ as above, i.e. of the form $(y, z)$, and let $\phi=\phi^{(\varepsilon)}$ be defined by (7.21), so in particular $\phi$ is $\pi$-invariant. In the proof of Proposition 7.1 we showed that there exists $\delta_{0} \in(0,1)$ such that for any $\delta \in\left(0, \delta_{0}\right)$ and any $\varepsilon \in(0,1)$

$$
\phi(\tilde{\xi}) \leqslant 2 \delta, \quad \tau(\tilde{\xi})-\tau_{0} \leqslant 2 \delta \quad \text { and } \quad\left|\tau^{2}(\tilde{\xi})+h(\tilde{\xi})-\lambda\right|<\varepsilon \delta
$$

imply that ${ }^{\mathrm{sc}} H_{g} \phi$ satisfies (7.33), so

$$
{ }^{\mathrm{sc}} H_{g} \phi(\tilde{\xi}) \geqslant c_{0}>0 \text {. }
$$


We define $q$ as in (10.71). Then (10.77), hence (10.78)-(10.82) also hold. Since $\varepsilon>0$ can be taken arbitrarily small, we can choose it and $\delta \in\left(0, \delta_{0}\right)$ so that supp $e$ is a small neighborhood of $\exp \left(s^{\mathrm{sc}} H_{g}\right)\left(\hat{\pi}^{-1}\left(\xi_{0}\right)\right)$; in particular, $\pi_{0 b}(\operatorname{supp} e)$ is disjoint from $\mathrm{WF}_{\mathrm{Sc}}(u)$ for each $b$. We can then apply the compactness argument of Proposition 10.1 to prove (10.42) for the operators $A$, $B$, etc., defined in that proof, and conclude that $\xi_{0} \notin \mathrm{WF}_{\mathrm{Sc}}(u)$.

We now return to the general setting of not necessarily totally geodesic $\mathcal{C}$.

Proposition 10.7. - Suppose that $H$ is a many-body Hamiltonian satisfying (10.1). Let $u \in \mathcal{C}^{-\infty}(X), \lambda>0$. Given

$$
K \subset\left(\Sigma_{t}(\lambda) \cap{ }^{\mathrm{sc}} T_{C^{\prime}}^{*}(C ; X)\right) \backslash\left(R_{+}(\lambda) \cup R_{-}(\lambda) \cup \mathrm{WF}_{\mathrm{Sc}}((H-\lambda) u)\right)
$$

compact there exist constants $C_{0}>0, \delta_{0}>0$ such that the following holds. If $\xi_{0}=\left(z_{0}, \tau_{0}, \nu_{0}\right) \in K$ and for some $0<\delta<\delta_{0}, C_{0} \delta \leqslant \varepsilon<1$ and for all $\alpha=(y, z, \tau, \mu, \nu) \in{ }^{\mathrm{sc}} T_{\partial X}^{*} X \cap \Sigma_{\Delta-\lambda}$

$$
\begin{aligned}
\alpha & \in{ }^{\mathrm{sc}} T_{C_{b}^{\prime}}^{*} X \quad \text { and }\left|\pi_{0 a}^{e}\left(\alpha-\exp \left(-\delta^{\mathrm{sc}} H_{g}\right)\left(\hat{\pi}^{-1}\left(\xi_{0}\right)\right)\right)\right| \leqslant \varepsilon \delta \quad \text { and }|y(\alpha)| \leqslant \varepsilon \delta \\
& \Rightarrow \pi_{0 b}(\alpha) \notin \mathrm{WF}_{\mathrm{Sc}}(u),
\end{aligned}
$$

then $\xi_{0} \notin \mathrm{WF}_{\mathrm{Sc}}(u)$.

Remark 10.8. - In the estimate (10.87), ${ }^{\mathrm{sc}} H_{g}$ can be replaced by any $\mathcal{C}^{\infty}$ vector field which agrees with ${ }^{\text {sc }} H_{g}$ at the point $\hat{\pi}^{-1}\left(\xi_{0}\right)$, since flow to distance $\delta$ along a vector field only depends on the vector field evaluated at the initial point of the flow, up to committing an error $\mathcal{O}\left(\delta^{2}\right)$. In particular, it can be replaced by the vector field $W^{\sharp}$ defined below. Similarly, changing the initial point of the flow by $\mathcal{O}\left(\delta^{2}\right)$ will not affect the endpoint up to an error $\mathcal{O}\left(\delta^{2}\right)$. Thus, estimate (10.87) can be further rewritten, at the cost of changing $C_{0}$ again, as

$$
\begin{aligned}
& \alpha \in{ }^{\mathrm{sc}} T_{C_{b}^{\prime}}^{*} X \quad \text { and }\left|\pi_{0 a}^{e}\left(\exp \left(\delta W^{b}\right)(\alpha)\right)-\xi_{0}\right| \leqslant \varepsilon \delta \quad \text { and } \quad\left|y\left(\exp \left(\delta W^{b}\right)(\alpha)\right)\right| \leqslant \varepsilon \delta \\
& (10.88) \Rightarrow \pi_{0 b}(\alpha) \notin \mathrm{WF}_{\mathrm{Sc}}(u) ;
\end{aligned}
$$

here we also interchanged the roles of the intial and final points of the flow. This relates (10.88) to $(10.50)$.

Proof. - The proof is very similar to the previous ones and now the positive commutator construction follows that of [40, Proposition 15.2] in three-body scattering. Thus, we take local coordinates as above, i.e. of the form $(y, z)$ with $C_{b}$ defined by linear equations in $y$. Then we construct $\omega_{0} \in \mathcal{C}^{\infty}\left({ }^{\mathrm{sc}} T_{C^{\prime}}^{*}(C ; X)\right)$ (defined near $\left.\xi_{0}\right)$ to measure the squared distance from integral curves of

$$
W^{\sharp}=2 \tau \nu \cdot \partial_{\nu}-2 \tilde{h} \partial_{\tau}+H_{\tilde{h}} ;
$$

this is achieved by solving a Cauchy problem as in [40] and in (7.12) here. (Indeed, an approximate construction, like that of $\omega_{0}$ in the normal case discussed above, would also work). Then we extend $\omega_{0}$ to a function on ${ }^{\mathrm{sc}} T_{\partial X}^{*} X$ (using the coordinates $(y, z, \tau, \mu, \nu)$ near $\partial X$ ), let

$$
\omega=\omega_{0}+|y|^{2}, \quad \phi=\tau_{0}-\tau+\frac{1}{\varepsilon^{2} \delta} \omega
$$

and define $q$ as in (10.14). The difference in the powers of $\varepsilon$ and $\delta$ in this definition of $\phi$ in the (general) tangential setting and that in the normal case (given in (10.70)) arises since in the normal setting $\omega$ approximates the fourth power of the distance from the generalized bicharacteristics while here it approximates the squared distance. The estimates on ${ }^{\mathrm{sc}} H_{g} \phi$ are 
just as in [40, Proposition 15.2], see also the proof of Proposition 7.1 here in the similar totally geodesic setting (the estimates are simply better but not different in nature under the totally geodesic assumption since now we do not have (7.4)), giving a slightly better result than in the totally geodesic normal case: it is $\delta / \varepsilon$, not $\delta / \varepsilon^{2}$, that has to be bounded from above by an appropriate positive constant. The difference arises as the model integral curves in the tangential setting are closer to the actual ones than in the normal setting. Thus, one obtains (10.26) here as well. The functional analysis part, under the assumption that there are no bound states, is exactly as in the normal case.

An argument of Melrose-Sjöstrand [23,24], see also [12, Chapter XXIV] and [18] allows us to conclude our main result concerning the singularities of generalized eigenfunctions of $H$. Here we concentrate on totally geodesic $\mathcal{C}$ (since that is the case in Euclidean scattering), in which case the more delicate tangential propagation argument of Melrose-Sjöstrand is not necessary. The proof presented below essentially follows Lebeau's paper [18, Proposition VII.1]. We thus have the following theorem.

THEOREM 10.9. - Let $(X, \mathcal{C})$ be a locally locally linearizable many-body space, and ssuppose that $H$ is a many-body Hamiltonian satisfying (10.1). Let $u \in \mathcal{C}^{-\infty}(X), \lambda>0$. Then $\mathrm{WF}_{\mathrm{Sc}}(u) \backslash \mathrm{WF}_{\mathrm{Sc}}((H-\lambda) u)$ is a union of maximally extended generalized broken bicharacteristics of $\Delta-\lambda$ in $\dot{\Sigma} \backslash \mathrm{WF}_{\mathrm{Sc}}((H-\lambda) u)$.

Proof. - We start by remarking that for every $V \subset \dot{\Sigma}$ and $\xi \in V$, the set $\mathcal{R}$ of generalized broken bicharacteristics $\gamma$ defined on open intervals including 0 , satisfying $\gamma(0)=\xi$, and with image in $V$, has a natural partial order, namely if $\gamma:(\alpha, \beta) \rightarrow V, \gamma^{\prime}:\left(\alpha^{\prime}, \beta^{\prime}\right) \rightarrow V$, then $\gamma \leqslant \gamma^{\prime}$ if the domains satisfy $(\alpha, \beta) \subset\left(\alpha^{\prime}, \beta^{\prime}\right)$ and $\gamma=\left.\gamma^{\prime}\right|_{(\alpha, \beta)}$. Moreover, any non-empty totally ordered subset has an upper bound: one can take the generalized broken bicharacteristic with domain given by the union of the domains of those in the totally ordered subset, and which extends these, as an upper bound. Hence, by Zorn's lemma, if $\mathcal{R}$ is not empty, it has a maximal element. Note that we can also work with intervals of the form $(\alpha, 0], \alpha<0$, instead of open intervals.

We only need to prove that for every $\xi_{0} \in \mathrm{WF}_{\mathrm{Sc}}(u) \backslash \mathrm{WF}_{\mathrm{Sc}}((H-\lambda) u)$ there exists a generalized broken bicharacteristic $\gamma:\left[-\varepsilon_{0}, \varepsilon_{0}\right] \rightarrow \dot{\Sigma}, \varepsilon_{0}>0$, with $\gamma(0)=\xi_{0}$ and such that $\gamma(t) \in \mathrm{WF}_{\mathrm{Sc}}(u) \backslash \mathrm{WF}_{\mathrm{Sc}}((H-\lambda) u)$ for $t \in\left[-\varepsilon_{0}, \varepsilon_{0}\right]$. In fact, once this statement is shown, taking $V=\mathrm{WF}_{\mathrm{Sc}}(u) \backslash \mathrm{WF}_{\mathrm{Sc}}((H-\lambda) u), \xi=\xi_{0}$, in the argument of the previous paragraph, we see that $\mathcal{R}$ is non-empty, hence has a maximal element. We need to show that such an element, $\gamma:(\alpha, \beta) \rightarrow \dot{\Sigma}$, is maximal in $\dot{\Sigma} \backslash \mathrm{WF}_{\mathrm{Sc}}((H-\lambda) u)$ as well, i.e. if we take $V=\dot{\Sigma} \backslash \mathrm{WF}_{\mathrm{Sc}}((H-$ $\lambda) u), \xi=\xi_{0}$ in the first paragraph. But if $\gamma^{\prime}:\left(\alpha^{\prime}, \beta^{\prime}\right) \rightarrow \dot{\Sigma}$ is any proper extension of $\gamma$, with say $\alpha^{\prime}<\alpha$, with image in $\dot{\Sigma} \backslash \mathrm{WF}_{\mathrm{Sc}}((H-\lambda) u)$, then $\gamma^{\prime}(\alpha) \in \mathrm{WF}_{\mathrm{Sc}}(u)$ since $\mathrm{WF}_{\mathrm{Sc}}(u)$ is closed, and $\gamma$ maps into it, hence by our assumption there is a generalized broken bicharacteristic $\tilde{\gamma}:\left(\alpha-\varepsilon^{\prime}, \alpha+\varepsilon^{\prime}\right) \rightarrow \mathrm{WF}_{\mathrm{Sc}}(u) \backslash \mathrm{WF}_{\mathrm{Sc}}((H-\lambda) u), \varepsilon^{\prime}>0, \tilde{\gamma}(\alpha)=\gamma^{\prime}(\alpha)$; piecing together $\left.\tilde{\gamma}\right|_{\left(\alpha-\varepsilon^{\prime}, \alpha\right]}$ and $\gamma$, directly from Definition 6.2, gives a generalized broken bicharacteristic which is a proper extension of $\gamma$, with image in $\mathrm{WF}_{\mathrm{Sc}}(u) \backslash \mathrm{WF}_{\mathrm{Sc}}((H-\lambda) u)$, contradicting the maximality of $\gamma$.

Indeed, it suffices to show that for any $a$, if

$$
\xi_{0} \in \mathrm{WF}_{\mathrm{Sc}}(u) \backslash \mathrm{WF}_{\mathrm{Sc}}((H-\lambda) u) \quad \text { and } \quad \xi_{0} \in{ }^{\mathrm{sc}} T_{C_{a}^{\prime}}^{*}\left(C_{a} ; X\right)
$$

then

(10.92) there exists a generalized broken bicharacteristic $\gamma:\left[-\varepsilon_{0}, 0\right] \rightarrow \dot{\Sigma}, \quad \varepsilon_{0}>0$,

$$
\gamma(0)=\xi_{0}, \quad \gamma(t) \in \mathrm{WF}_{\mathrm{Sc}}(u) \backslash \mathrm{WF}_{\mathrm{Sc}}((H-\lambda) u), \quad t \in\left[-\varepsilon_{0}, 0\right],
$$


for the existence of a generalized broken bicharacteristic on $\left[0, \varepsilon_{0}\right]$ can be demonstrated similarly by replacing the forward propagation estimates by backward ones, and, directly from Definition 6.2, piecing together the two generalized broken bicharacteristics gives one defined on $\left[-\varepsilon_{0}, \varepsilon_{0}\right]$.

We proceed to prove that (10.91) implies (10.92) by induction on $a$. For $a=0$, this is certainly true by Proposition 10.6, since only elements of $C \in \mathcal{C}$ in a small neighborhood of $\xi_{0}$ must be totally geodesic for the proof to go through, and $C_{0}$ is the only such element if the neighborhood of $\xi_{0} \in{ }^{\mathrm{sc}} T_{C_{0}^{\prime}}^{*}\left(C_{0} ; X\right)={ }^{\mathrm{sc}} T_{C_{0}^{\prime}}^{*} X$ is chosen sufficiently small (in fact, this is really just Melrose's propagation theorem [22]).

So suppose that $(10.91) \Rightarrow(10.92)$ has been proved for all $b$ with $C_{a} \subsetneq C_{b}$ and that $\xi_{0} \in$ $\Sigma_{n}(\lambda) \cap{ }^{\mathrm{sc}} T_{C_{a}^{\prime}}^{*}\left(C_{a} ; X\right)$ satisfies (10.91). We use the notation of the proof of Proposition 10.1 below. Let $U \subset \bigcup_{C_{a} \subset C_{b}}{ }^{\text {sc }} T_{C_{b}^{\prime}}^{*}\left(C_{b} ; X\right)$ be a neighborhood of $\xi_{0}=\left(0, z_{0}, \tau_{0}, \nu_{0}\right)$ in $\dot{\Sigma}$ which is given by equations of the form $|y|<\delta^{\prime},\left|z-z_{0}\right|<\delta^{\prime},\left|\tau-\tau_{0}\right|<\delta^{\prime},\left|\nu-\nu_{0}\right|<\delta^{\prime}, \delta^{\prime}>0$, such that ${ }^{\mathrm{sc}} H_{g} \eta>0$ on $\hat{\pi}^{-1}(U)$ and $U \cap \mathrm{WF}_{\mathrm{Sc}}((H-\lambda) u)=\emptyset$. Such a neighborhood exists since $\xi_{0} \notin \mathrm{WF}_{\mathrm{Sc}}((H-\lambda) u)$ and ${ }^{\mathrm{sc}} H_{g} \eta\left(\tilde{\xi}_{0}\right)=\lambda-\tau_{0}^{2}-\tilde{h}\left(z_{0}, \nu_{0}\right)>0$ for every $\tilde{\xi}_{0} \in \hat{\pi}^{-1}\left(\xi_{0}\right)$. Also let $U^{\prime}$ be a subset of $U$ defined by replacing $\delta^{\prime}$ by a smaller $\delta^{\prime \prime}>0$, and let $\varepsilon_{0}>0$ be such that for any generalized broken bicharacteristic $\gamma$ with $\gamma(0) \in U^{\prime},\left.\gamma\right|_{\left[-\varepsilon_{0}, \varepsilon_{0}\right]} \in U$. By Proposition 10.1, there is a sequence of points $\xi_{n} \in \dot{\Sigma}$ such that $\xi_{n} \in \mathrm{WF}_{\mathrm{Sc}}(u), \xi_{n} \rightarrow \xi_{0}$ as $n \rightarrow \infty$, and $\eta\left(\xi_{n}\right)<0$ for all $n$, so we may assume that $\xi_{n} \in U^{\prime}$ for all $n$. By the inductive hypothesis, for each $n$, there exists a generalized broken bicharcteristic

$$
\tilde{\gamma}_{n}:\left(-\varepsilon_{n}^{\prime}, 0\right] \rightarrow\left(\mathrm{WF}_{\mathrm{Sc}}(u) \backslash \mathrm{WF}_{\mathrm{Sc}}((H-\lambda) u)\right) \cap \bigcup_{C_{a \subsetneq C} C_{b}}{ }^{\mathrm{sc}} T_{C_{b}^{\prime}}^{*}\left(C_{b} ; X\right)
$$

with $\tilde{\gamma}_{n}(0)=\xi_{n}$. We now use the argument of the first paragraph of the proof with $V=\left(\mathrm{WF}_{\mathrm{Sc}}(u) \backslash \mathrm{WF}_{\mathrm{Sc}}((H-\lambda) u)\right) \cap \bigcup_{C_{a} \subsetneq C_{b}}{ }^{\mathrm{sc}} T_{C_{b}^{\prime}}^{*}\left(C_{b} ; X\right)$, and $\xi=\xi_{n}$. Thus, $\tilde{\gamma}_{n} \in \mathcal{R}$, which is hence non-empty, hence has a maximal element. We let

$$
\gamma_{n}:\left(-\varepsilon_{n}, 0\right] \rightarrow\left(\mathrm{WF}_{\mathrm{Sc}}(u) \backslash \mathrm{WF}_{\mathrm{Sc}}((H-\lambda) u)\right) \cap \bigcup_{C_{a} \subsetneq C_{b}}{ }^{\mathrm{sc}} T_{C_{b}^{\prime}}^{*}\left(C_{b} ; X\right)
$$

be a maximal element of $\mathcal{R}$; it may happen that $-\varepsilon_{n}=-\infty$.

We claim that $\varepsilon_{n} \geqslant \varepsilon_{0}$. For suppose that $\varepsilon_{n}<\varepsilon_{0}$. By Corollary 6.12, $\gamma_{n}$ extends to a generalized broken bicharacteristic on $\left[-\varepsilon_{n}, 0\right]$, we continue to denote this by $\gamma_{n}$. Since $\varepsilon_{n}<\varepsilon_{0}$, $\gamma_{n}$ is a generalized broken bicharacteristic with image in $U$; indeed the closure of the image is still in $U$. Taking into account that $\eta$ is increasing on generalized broken bicharacteristics in $U$ since ${ }^{\text {sc }} H_{g} \eta>0$ there, we conclude that $y\left(\gamma_{n}(t)\right) \cdot \mu\left(\gamma_{n}(t)\right)=\eta\left(\gamma_{n}(t)\right) \leqslant \eta\left(\gamma_{n}(0)\right)<0$ for $t \in\left[-\varepsilon_{n}, 0\right]$, hence $y\left(\gamma_{n}(t)\right) \neq 0$. Thus, $\gamma_{n}\left(-\varepsilon_{n}\right) \in \bigcup_{C_{a} \subsetneq C_{b}}{ }^{s c} T_{C_{b}^{\prime}}^{*}\left(C_{b} ; X\right)$. Moreover, $\gamma_{n}\left(-\varepsilon_{n}\right) \in \mathrm{WF}_{\mathrm{Sc}}(u)$ since $\mathrm{WF}_{\mathrm{Sc}}(u)$ is closed, and $\left.\gamma_{n}\right|_{\left(-\varepsilon_{n}, 0\right]}$ maps into it. Thus, by the inductive hypothesis, there is a generalized broken bicharacteristic,

$$
\tilde{\gamma}_{n}:\left(\alpha,-\varepsilon_{n}\right] \rightarrow\left(\mathrm{WF}_{\mathrm{Sc}}(u) \backslash \mathrm{WF}_{\mathrm{Sc}}((H-\lambda) u)\right) \cap \bigcup_{C_{a} \subsetneq C_{b}}{ }^{\mathrm{sc}} T_{C_{b}^{\prime}}^{*}\left(C_{b} ; X\right)
$$

with $\alpha<-\varepsilon_{n}, \tilde{\gamma}_{n}\left(-\varepsilon_{n}\right)=\gamma_{n}\left(-\varepsilon_{n}\right)$. Hence, piecing together $\tilde{\gamma}_{n}$ and $\gamma_{n}$ gives a generalized broken bicharacteristic mapping into $\left(\mathrm{WF}_{\mathrm{Sc}}(u) \backslash \mathrm{WF}_{\mathrm{Sc}}((H-\lambda) u)\right) \cap \bigcup_{C_{a} \subsetneq C_{b}}{ }^{{ }^{\mathrm{sc}}} T_{C_{b}^{\prime}}^{*}\left(C_{b} ; X\right)$ and extending $\gamma_{n}$, which contradicts the maximal property of $\gamma_{n}$. Thus, $\varepsilon_{n} \geqslant \varepsilon_{0}$ as claimed. 
By Proposition 6.11, applied with $K=\mathrm{WF}_{\mathrm{Sc}}(u)$, there is a subsequence of $\left.\gamma_{n}\right|_{\left[-\varepsilon_{0}, 0\right]}$ converging uniformly to a generalized broken bicharacteristic $\gamma:\left[-\varepsilon_{0}, 0\right] \rightarrow \operatorname{WF}_{\mathrm{Sc}}(u)$. In particular, $\gamma(0)=\xi_{0}$ and $\gamma(t) \in \mathrm{WF}_{\mathrm{Sc}}(u)$ for all $t \in\left[-\varepsilon_{0}, 0\right]$, providing the inductive step.

We now turn to $\xi_{0} \in \Sigma_{t}(\lambda) \cap{ }^{\mathrm{sc}} T_{C_{a}^{\prime}}^{*}\left(C_{a} ; X\right)$. If every element of $\mathcal{C}$ is totally geodesic, then due to Proposition 10.6, (10.91) $\Rightarrow(10.92)$. (Again, we only need that $C_{b}$ be totally geodesic for $C_{b}$ with $C_{a} \subset C_{b}$, since the result is local.)

The general case, when element of $\mathcal{C}$ are not totally geodesic, repeats the argument of MelroseSjöstrand, as presented in Lebeau's paper [18, Proposition VII.1]. We very briefly outline the proof below; the detailed version follows Lebeau's closely, with some changes in the notation. Let $U \subset \bigcup_{C_{a} \subset C_{b}}{ }^{\text {sc }} T_{C_{b}^{\prime}}^{*}\left(C_{b} ; X\right) \backslash \mathrm{WF}_{\mathrm{Sc}}((H-\lambda) u)$ be a neighborhood of $\xi_{0}, U_{0}$ a smaller neighborhood, as above. We take $\varepsilon_{0}>0$ small. Suppose that $0<\varepsilon<\varepsilon_{0}, \xi \in U_{0}$. Let

$$
\begin{aligned}
\mathcal{R}_{\xi, \varepsilon}^{1}= & \left\{\text { generalized broken bicharacteristics } \gamma:[-\varepsilon, 0] \rightarrow \mathrm{WF}_{\mathrm{Sc}}(u),\right. \\
& \left.\gamma(0)=\xi, \gamma(t) \notin \Sigma_{t}(\lambda) \cap{ }^{\mathrm{sc}} T_{C_{a}^{\prime}}^{*}\left(C_{a} ; X\right) \text { for } t \in(-\varepsilon, 0]\right\}, \\
\mathcal{R}_{\xi, \varepsilon}^{2}=\{ & \text { generalized broken bicharacteristics } \gamma:\left[-\varepsilon^{\prime}, 0\right] \rightarrow \mathrm{WF}_{\mathrm{Sc}}(u), \varepsilon^{\prime} \in(0, \varepsilon), \\
& \gamma(0)=\xi, \gamma(t) \notin \Sigma_{t}(\lambda) \cap{ }^{\mathrm{sc}} T_{C_{a}^{\prime}}^{*}\left(C_{a} ; X\right) \text { for } t \in\left(-\varepsilon^{\prime}, 0\right], \\
& \left.\gamma\left(-\varepsilon^{\prime}\right) \in \Sigma_{t}(\lambda) \cap{ }^{\mathrm{sc}} T_{C_{a}^{\prime}}^{*}\left(C_{a} ; X\right)\right\} .
\end{aligned}
$$

Moreover, reflecting the inequalities in (10.87), let

$$
B(\xi, \varepsilon)=\left\{\xi^{\prime} \in \dot{\Sigma}: \max \left\{\left|\pi_{0 a}^{e}\left(\xi^{\prime}\right)-\xi\right|,\left|y\left(\xi^{\prime}\right)\right|\right\}<\varepsilon\right\} .
$$

Let $C_{0}>0$ be as in Proposition 10.7. For $\xi \in \Sigma_{t}(\lambda) \cap{ }^{\mathrm{sc}} T_{C_{a}^{\prime}}^{*}\left(C_{a} ; X\right)$, let

$$
D(\xi, \varepsilon)=B\left(\exp \left(-\varepsilon^{\mathrm{sc}} H_{g}\right)\left(\hat{\pi}^{-1}(\xi)\right), C_{0} \varepsilon^{2}\right) \cap \mathrm{WF}_{\mathrm{Sc}}(u),
$$

and for $\xi \notin \Sigma_{t}(\lambda) \cap{ }^{\mathrm{sc}} T_{C_{a}^{\prime}}^{*}\left(C_{a} ; X\right)$, let

$$
D(\xi, \varepsilon)=\left\{\gamma(-\varepsilon): \gamma \in \mathcal{R}_{\xi, \varepsilon}^{1}\right\}
$$

$$
\cup\left\{B\left(\exp \left(-\left(\varepsilon-\varepsilon^{\prime}\right)^{\mathrm{sc}} H_{g}\right)\left(\hat{\pi}^{-1}\left(\gamma\left(\varepsilon^{\prime}\right)\right), C_{0}\left(\varepsilon-\varepsilon^{\prime}\right)^{2}\right)\right) \cap \mathrm{WF}_{\mathrm{Sc}}(u): \gamma \in \mathcal{R}_{\xi, \varepsilon}^{2}\right\} .
$$

The reason for introducing $D(\xi, \varepsilon)$ is that it is a good candidate for the beginning point of a generalized broken bicharacteristic segment in $\mathrm{WF}_{\mathrm{Sc}}(u)$, defined over an interval of length $\varepsilon$, and ending in $\xi$.

Indeed, for $\xi \in \Sigma_{t}(\lambda) \cap{ }^{\mathrm{sc}} T_{C_{a}^{\prime}}^{*}\left(C_{a} ; X\right) \cap \mathrm{WF}_{\mathrm{Sc}}(u)$, we deduce from Proposition 10.7 that $D(\xi, \varepsilon) \neq \emptyset$. For $\xi \in \mathrm{WF}_{\mathrm{Sc}}(u) \backslash\left(\Sigma_{t}(\lambda) \cap{ }^{\mathrm{sc}} T_{C_{a}^{\prime}}^{*}\left(C_{a} ; X\right)\right)$, by the inductive hypothesis, the previous part of the proof concerning $\Sigma_{n}(\lambda) \cap{ }^{{ }^{c}} T_{C_{a}^{\prime}}^{*}\left(C_{a} ; X\right)$, and the first two paragraphs with $V=\mathrm{WF}_{\mathrm{Sc}}(u) \backslash\left(\left(\Sigma_{t}(\lambda) \cap{ }^{\mathrm{sc}} T_{C_{a}^{\prime}}^{*}\left(C_{a} ; X\right)\right) \cup \mathrm{WF}_{\mathrm{Sc}}(H-\lambda) u\right), \xi=\xi_{0}$, there is a maximally extended generalized broken bicharacteristic $\gamma$ with image in $V$. By the argument of the second paragraph, this is either defined on all of $[-\varepsilon, 0]$, or only on $\left(-\varepsilon^{\prime}, 0\right]$ with $0<\varepsilon^{\prime}<\varepsilon$, in which case $\gamma\left(-\varepsilon^{\prime}\right) \in \Sigma_{t}(\lambda) \cap{ }^{\mathrm{sc}} T_{C_{a}^{\prime}}^{*}\left(C_{a} ; X\right)$, hence again by Proposition 10.7 we conclude that $D(\xi, \varepsilon) \neq \emptyset$. Thus, for all $\xi \in U \cap \mathrm{WF}_{\mathrm{Sc}}(u)$ we have deduced $D(\xi, \varepsilon) \neq \emptyset$.

For each integer $N \geqslant 1$ now we define a sequence of $2^{N}+1$ points $\xi_{j, N}, j \in \mathbb{N}, 0 \leqslant j \leqslant 2^{N}$, which will be used to construct points $\gamma\left(-j 2^{-N} \varepsilon_{0}\right)$ on the desired generalized broken bicharacteristic $\gamma:\left[-\varepsilon_{0}, 0\right] \rightarrow \mathrm{WF}_{\mathrm{Sc}}(u)$ through $\xi_{0}$. Namely, let $\varepsilon=2^{-N} \varepsilon_{0}, \xi_{0, N}=\xi_{0}$, and choose $\xi_{j+1, N} \in D\left(\xi_{j, N}, \varepsilon\right)$. Let $\mathcal{J}_{N}=\left\{-j 2^{-N} \varepsilon_{0}: 0 \leqslant j \leqslant 2^{N}\right\} \subset\left[-\varepsilon_{0}, 0\right], \mathcal{J}=\bigcup_{N=1}^{\infty} \mathcal{J}_{N}$. We write $\gamma_{N}(t)=\xi_{j, N}$ for $t=-j 2^{-N} \varepsilon_{0}$. For each $t \in \mathcal{J}$, the sequence $\gamma_{N}(t)$ (defined for 
large $N$ ) stays in a compact set. Hence there exists a subsequence $\gamma_{N_{k}}$ such that for all $t \in \mathcal{J}$, $\gamma_{N_{k}}(t)$ converges to some $\gamma(t)$.

This defines $\gamma:\left[-\varepsilon_{0}, 0\right] \rightarrow \mathrm{WF}_{\mathrm{Sc}}(u)$ at elements of $\mathcal{J}$. One can check exactly as in Lebeau's proof (which we have been following very closely) that $\gamma$ extends to a continuous map defined on $\left[-\varepsilon_{0}, 0\right]$, and that it is a generalized broken bicharacteristic. This completes the inductive step for tangential points $\xi_{0} \in \Sigma_{t}(\lambda) \cap{ }^{\mathrm{sc}} T_{C_{a}^{\prime}}^{*}\left(C_{a} ; X\right)$ in the non-totally geodesic setting, hence the proof of the theorem.

We remark that the result is optimal as can be seen by considering the Euclidean setting, taking potentials singular at a specified $C_{a}$, thereby placing ourselves into the three-body framework. As [37] shows, singularities do reflect in all permissible directions in general, the reflection being governed to top order by the (two-body) S-matrix of the subsystem.

\section{The resolvent}

Before we can turn Theorem 10.9 into a result on the wave front relation of the S-matrix, we need to analyze the resolvent. More precisely, we need to understand the boundary values

$$
R(\lambda \pm i 0)=(H-(\lambda \pm i 0))^{-1}
$$

of the resolvent at the real axis in a microlocal sense. To do so, we also need estimates at the radial sets $R_{ \pm}(\lambda)$. Since the rescaled Hamilton vector field of the metric $g$ vanishes at $R_{+}(\lambda) \cup R_{-}(\lambda)$, the estimates must utilize the weights $x^{-l-1}$ themselves. In this sense they are delicate, but on the other hand they only involve $x$ and its sc-microlocal dual variable $\tau$, so they do not need to reflect the geometry of $\mathcal{C}$. The best known positive commutator estimate is the Mourre estimate, originally proved by Perry, Sigal and Simon in Euclidean many-body scattering [27], in which one takes $q=x^{-1} \tau$ with the notation of Section 9. Since it is easy to analyze the commutator of powers of $x$ with $H$ (in particular, they commute with $V$ ), the functional calculus allows one to obtain microlocal estimates from these, as was done by Gérard, Isozaki and Skibsted $[6,7]$. Thus, nearly all the technical results in this section can be proved, for example, by using the Mourre estimate and Theorem 10.9. In particular, apart from the propagation statements, they are well-known in Euclidean many-body scattering. The generalization of these Euclidean results to our geometric setting is straightforward; the arguments essentially follow those in three-body scattering that were used in [40].

We first state the weak form of the limiting absorption principle, namely that for $f \in \dot{\mathcal{C}}^{\infty}(X)$, $R(\lambda \pm i t) f, t>0$, has a limit in $H_{\mathrm{sc}}^{m, l}(X), m$ arbitrary, $l<-1 / 2$, as $t \rightarrow 0$. To simplify the asymptotic expansions of $R(\lambda \pm i 0) f$ which we also describe, for $\lambda>0$ we introduce the functions

$$
\alpha_{ \pm}=\alpha_{ \pm, \lambda}= \pm \frac{V}{2 \sqrt{\lambda} x} \in \mathcal{C}^{\infty}\left(X \backslash C_{0, \text { sing }}\right),
$$

and the set of polyhomogeneous functions $\mathcal{A}_{\mathrm{phg}}^{\mathcal{K}}\left(X \backslash C_{0, \text { sing }}\right)$ on $X \backslash C_{0 \text {,sing }}$ with index set

$$
\mathcal{K}=\{(m, p): m, p \in \mathbb{N}, p \leqslant 2 m\} .
$$

Recall from [21] that $v \in \mathcal{A}_{\mathrm{phg}}^{\mathcal{K}}\left(X \backslash C_{0, \text { sing }}\right)$ means that $v$ is $\mathcal{C}^{\infty}$ in the interior of $X$ and it has a full asymptotic expansion at $C_{0}^{\prime}$ which in local coordinates $(x, y)$ take the form

$$
v(x, y) \sim \sum_{j=0}^{\infty} \sum_{r \leqslant 2 j} x^{j}(\log x)^{r} a_{j, r}(y), \quad a_{j, r} \in \mathcal{C}^{\infty}\left(C_{0}^{\prime}\right) .
$$


Thus, $v \in \mathcal{C}^{0}\left(X \backslash C_{0, \text { sing }}\right)$ and $\left|v(x, y)-a_{0,0}(y)\right| \leqslant C x|\log x|^{2}$.

THEOREM 11.1. - Suppose that $H$ is a many-body Hamiltonian satisfying (10.1), $\lambda>0$. Let $f \in \dot{\mathcal{C}}^{\infty}(X), u_{t}^{ \pm}=R(\lambda \pm i t) f, t>0$. Then $u_{t}^{ \pm}$has a limit $u_{ \pm}=R(\lambda \pm i 0) f$ in $H_{\mathrm{sc}}^{m, l}(X)$, $l<-1 / 2$, as $t \rightarrow 0$. In addition,

$$
\mathrm{WF}_{\mathrm{Sc}}\left(u_{ \pm}\right) \subset R_{\mp}(\lambda) .
$$

If $V$ is short-range, i.e. $V \in x^{2} \mathcal{C}^{\infty}\left(X \backslash C_{0, \text { sing }}\right)$, then

$$
u_{ \pm}=\mathrm{e}^{ \pm i \sqrt{\lambda} / x} x^{(n-1) / 2} v_{ \pm}, \quad v_{ \pm} \in \mathcal{C}^{\infty}\left(X \backslash C_{0, \operatorname{sing}}\right)
$$

while if $V$ is long-range, i.e. $V$ merely satisfies (6.2), then

$$
u_{ \pm}=\mathrm{e}^{ \pm i \sqrt{\lambda} / x} x^{(n-1) / 2+i \alpha_{ \pm}} v_{ \pm}, \quad v_{ \pm} \in \mathcal{A}_{\mathrm{phg}}^{\mathcal{K}}\left(X \backslash C_{0, \text { sing }}\right) .
$$

Remark 11.2. - The first statement in the theorem also holds if we merely assume $f \in$ $H_{\mathrm{sc}}^{m, l^{\prime}}(X)$ with $l^{\prime}>1 / 2$, but then $\mathrm{WF}_{\mathrm{Sc}}\left(u_{ \pm}\right)$has to be replaced by the filtered wave front set $\mathrm{WF}_{\mathrm{Sc}}^{m, l^{\prime}-1}\left(u_{ \pm}\right)$. Moreover, $R(\lambda \pm i 0)$ give continuous operators from $H_{\mathrm{sc}}^{m, l^{\prime}}(X)$ to $H_{\mathrm{sc}}^{m+2, l}(X)$.

Proof. - This result is a weak form of the limiting absorption principle and can be proved by a Mourre-type estimate. In the Euclidean setting, it is a combination of the Mourre estimate, proved by Perry, Sigal and Simon [27], and its microlocalized version obtained by Gérard, Isozaki and Skibsted [6]. In the geometric setting, the Mourre estimate describes the commutator of $H$ with a self-adjoint first order differential operator $A \in x^{-1} \operatorname{Diff}_{\mathrm{sc}}^{1}(X)$ such that $A-x D_{x} \in \operatorname{Diff}_{\mathrm{sc}}^{1}(X)$ (this is of course a restriction only at $\partial X)$. Namely, it says that for $\phi \in \mathcal{C}_{c}^{\infty}(\mathbb{R} ;[0,1])$ supported sufficiently close to $\lambda$, we have

$$
i \phi(H)[A, H] \phi(H) \geqslant 2(\lambda-\varepsilon) \phi(H)^{2}+R, \quad \varepsilon>0,
$$

where $R \in \Psi_{\mathrm{Sc}}^{-\infty, 1}(X, \mathcal{C})$, hence compact on $L_{\mathrm{sc}}^{2}(X)$. It was proved in the geometric threebody setting (with an appropriate adjustment to allow bound states of subsystems) in [40], following the Euclidean argument of Froese and Herbst [5]. The proof given there goes through essentially unchanged for more than three bodies. Under our assumption (10.1), the symbolic commutator calculation in the scattering calculus, ${ }^{\mathrm{sc}} H_{g}\left(x^{-1} \tau\right)+2 g \in x \mathcal{C}^{\infty}\left({ }^{\mathrm{sc}} T^{*} X\right)$, and a slight modification of Corollary 9.7, prove the Mourre estimate. The argument of [27] then proves the existence of the limits $u_{ \pm}$in $H_{\mathrm{sc}}^{0, l}(X), l<-1 / 2$, and $(H-\lambda) u_{ \pm}=f \in \dot{\mathcal{C}}^{\infty}(X)$ shows that the same holds in $H_{\mathrm{sc}}^{m, l}(X)$ for every $m$ and for every $l<-1 / 2$.

To show the flavor of the arguments, we prove here a version of the estimate of Gerard, Isozaki and Skibsted [6]. Such arguments as this can be combined to prove the limiting absorption principle without a direct use of the Mourre estimate as was done in the geometric two-body type setting by Melrose [22] and in the geometric three-body setting in [40]. Here, however, we concentrate on proving the wave front set result. The major difference between the propagation estimates of the previous section and the ones near $R_{ \pm}(\lambda)$ is that ${ }^{\mathrm{sc}} H_{g}$ is radial at $R_{+}(\lambda) \cup R_{-}(\lambda)$ : it has the form $2 \tau x \partial_{x}$. Thus, we need to use a weight $x^{-l-1}$ to obtain a positive symbol estimate. So for $l>-1$, let

$$
q=x^{-l-1} \chi(\tau) \tilde{\psi}(x) \geqslant 0
$$

where $\tilde{\psi} \in \mathcal{C}_{c}^{\infty}(\mathbb{R})$ is identically 1 near 0 and is supported in a bigger neighborhood of 0 (it is simply a cutoff near $\partial X), \chi \in \mathcal{C}_{c}^{\infty}(\mathbb{R} ;[0,1])$ vanishes on $(-\infty, \sqrt{\lambda}-2 \varepsilon)$, identically 1 on 
$(\sqrt{\lambda}-\varepsilon, \infty), \varepsilon>0, \chi^{\prime} \geqslant 0$, and $\chi$ vanishes with all derivatives at every $t$ with $\chi(t)=0$. Then for sufficiently small $\delta>0,|g-\lambda|=\left|\tau^{2}+h-\lambda\right|<\delta$ implies

$$
\begin{aligned}
& { }^{\mathrm{sc}} H_{g} q=-2\left((l+1) \tau \chi(\tau)+h \chi^{\prime}(\tau)\right) x^{-l-1} \leqslant-b^{2} x^{-l-1}, \\
& b=\left(2(l+1) \tau \chi(\tau)+\left(\lambda-\tau^{2}\right) \chi^{\prime}(\tau) / 2\right)^{1 / 2} .
\end{aligned}
$$

Thus, both $x^{l+1} q$ and $x^{l+1} b$ are $\pi$-invariant. Let $A \in \Psi_{\mathrm{Sc}}^{-\infty,-l-1}(X, \mathcal{C})$ be a quantization of $q$ as in Lemma 9.1, except that now $q$ is not supported in a single coordinate chart, so we need to define $A$ as the sum of localized operators (of course, this is not necessary in the actual Euclidean setting). Thus, roughly speaking, $A$ is the product of a quantization of $q$ and $\psi_{0}(H)$, $\psi_{0} \in \mathcal{C}_{c}^{\infty}(\mathbb{R})$. The fact that $q \in x^{-l-1} \mathcal{C}^{\infty}\left({ }^{\mathrm{sc}} T^{*} X\right)$ does not cause any trouble, and the argument of Corollary 9.7 shows that for $\psi \in \mathcal{C}_{c}^{\infty}(\mathbb{R} ;[0,1])$ supported sufficiently close to $\lambda$ we have

$$
i x^{l+1 / 2} \psi(H)\left[A^{*} A, H\right] \psi(H) x^{l+1 / 2} \geqslant x^{l+1 / 2}\left(\left(2-\varepsilon^{\prime}\right) B^{*} B+F\right) x^{l+1 / 2}, \quad \varepsilon^{\prime}>0,
$$

where

$$
\begin{gathered}
F \in \Psi_{\mathrm{Sc}}^{-\infty,-2 l}(X, \mathcal{C}), \mathrm{WF}_{\mathrm{Sc}}^{\prime}(F) \subset \operatorname{supp}\left(x^{l+1} q\right) \\
B \in \Psi_{\mathrm{Sc}}^{-\infty,-l-1 / 2}(X, \mathcal{C}), \hat{B}_{a,-l-1 / 2}(\zeta)=b(\zeta) q(\zeta)^{1 / 2} \psi\left(\hat{H}_{a, 0}(\zeta)\right) .
\end{gathered}
$$

Let

$$
A_{0}=A \psi(H) \in \Psi_{\mathrm{Sc}}^{-\infty,-l-1}(X, \mathcal{C}) .
$$

Since

$$
\left\langle u_{t}^{+}, i\left[A_{0}^{*} A_{0}, H\right] u_{t}^{+}\right\rangle=-2 \operatorname{Im}\left\langle u_{t}^{+}, A_{0}^{*} A_{0}(H-(\lambda+i t)) u_{t}^{+}\right\rangle-2 t\left\|A_{0} u_{t}^{+}\right\|^{2},
$$

we conclude that

$$
\left\|B u_{t}^{+}\right\|^{2}+2 t\left\|A_{0} u_{t}^{+}\right\|^{2} \leqslant\left|\left\langle u_{t}^{+}, F u_{t}^{+}\right\rangle\right|+2\left|\left\langle u_{t}^{+}, A_{0}^{*} A_{0}(H-(\lambda+i t)) u_{t}^{+}\right\rangle\right| .
$$

Since $t>0$, the second term on the left hand side can be dropped. Since $u_{t}^{+} \rightarrow u_{+}$in $H_{\mathrm{sc}}^{0, l^{\prime}}(X)$ for $l^{\prime}<-1 / 2$, we conclude that for $l \in(-1,-1 / 2)$ the right hand side stays bounded as $t \rightarrow 0$. Thus, $B u_{t}^{+}$is uniformly bounded in $L_{\mathrm{sc}}^{2}(X)$, and as $u_{t}^{+} \rightarrow u_{+}$in $H_{\mathrm{sc}}^{0, l^{\prime}}(X)$, we conclude that $B u_{+} \in L_{\mathrm{sc}}^{2}(X)$. But then (11.13) shows that for any $\zeta$ with $q(\zeta) \neq 0$, we have $\zeta \notin \mathrm{WF}_{\mathrm{Sc}}^{m, l+1 / 2}\left(u_{+}\right)$for every $m$. This proves that the incoming radial set, $R_{+}(\lambda)$, is disjoint from $\mathrm{WF}_{\mathrm{Sc}}^{m, l+1 / 2}\left(u_{+}\right), l+1 / 2 \in(-1 / 2,0)$. Iterating the argument, as in the proof of Proposition 10.1, gives that $\mathrm{WF}_{\mathrm{Sc}}\left(u_{+}\right) \cap R_{+}(\lambda)=\emptyset$. Since $\mathrm{WF}_{\mathrm{Sc}}\left(u_{+}\right)$is closed, the same conclusion holds for a neighborhood of $R_{+}(\lambda)$. Finally, as all generalized broken bicharacteristics of $\Delta-\lambda$ tend to $R_{+}(\lambda)$ as $t \rightarrow-\infty$ and $(H-\lambda) u_{+}=f \in \dot{\mathcal{C}}^{\infty}(X)$, the propagation of singularities theorem, Theorem 10.9, implies that $\mathrm{WF}_{\mathrm{Sc}}\left(u_{+}\right) \subset R_{-}(\lambda)$. The existence of the asymptotic expansions is a local question, so at $C_{0}^{\prime}$ we can work in the scattering calculus to prove it, see [35] for details of the proof.

A pairing argument immediately shows $R(\lambda \pm i 0) v$ also exists for distributions $v \in \mathcal{C}^{-\infty}(X)$ with wave front set disjoint from the incoming and outgoing radial set respectively. Combining it with the propagation theorem, Theorem 10.9, we can deduce the following result; as usual, we assume that $(X, \mathcal{C})$ is locally linearizable. 
THEOREM 11.3. - Suppose that $H$ is a many-body Hamiltonian satisfying (10.1), $\lambda>0$. Suppose also that $v \in \mathcal{C}^{-\infty}(X)$ and $\mathrm{WF}_{\mathrm{Sc}}(v) \cap R_{+}(\lambda)=\emptyset$. Let $u_{t}^{+}=R(\lambda+i t) v, t>0$. Then $u_{t}^{+}$has a limit $u_{+}=R(\lambda+i 0) v$ in $\mathcal{C}^{-\infty}(X)$ as $t \rightarrow 0$ and $\mathrm{WF}_{\mathrm{Sc}}\left(u_{+}\right) \cap R_{+}(\lambda)=\emptyset$. Moreover, if $\xi \in \dot{\Sigma} \backslash R_{-}(\lambda)$ and every maximally backward extended generalized broken bicharacteristic, $\gamma:\left(-\infty, t_{0}\right] \rightarrow \dot{\Sigma}$, with $\gamma\left(t_{0}\right)=\xi$ is disjoint from $\mathrm{WF}_{\mathrm{Sc}}(v)$, then $\xi \notin \mathrm{WF}_{\mathrm{Sc}}\left(u_{+}\right)$. The result also holds with $R_{+}(\lambda)$ and $R_{-}(\lambda)$ interchanged, $R(\lambda+i t)$ replaced by $R(\lambda-i t),\left(-\infty, t_{0}\right]$ by $\left[t_{0}, \infty\right)$ and correspondingly 'backward extended' by 'forward extended'.

Proof. - As mentioned above, the first part follows from the self-adjointness of $H$, so that for $t>0, v \in \mathcal{C}^{-\infty}(X), f \in \dot{\mathcal{C}}^{\infty}(X)$, we have $v(R(\lambda+i t) f)=R(\lambda+i t) v(f)$; recall that the distributional pairing is the real pairing, not the complex (i.e. $L^{2}$ ) one. The wave front statement of Theorem 11.1 and the assumption on $v$ show the existence of the limit $u_{+}=R(\lambda+i 0) v$ in $\mathcal{C}^{-\infty}(X)$ and that in addition $\mathrm{WF}_{\mathrm{Sc}}^{m, l}\left(u_{+}\right) \cap R_{+}(\lambda)=\emptyset$ for every $l<-1 / 2$. The positive commutator argument of Theorem 11.1 then applies and shows that $\mathrm{WF}_{\mathrm{Sc}}\left(u_{+}\right) \cap R_{+}(\lambda)=\emptyset$. In the Euclidean setting these results follow from a microlocalized version of the Mourre estimate due to Gérard, Isozaki and Skibsted [7]; see [9] for a detailed argument.

Finally, since $\mathrm{WF}_{\mathrm{Sc}}\left(u_{+}\right)$is closed, a neighborhood of $R_{+}(\lambda)$ in $\dot{\Sigma}$ is disjoint from $\mathrm{WF}_{\mathrm{Sc}}\left(u_{+}\right)$. Since all generalized broken bicharacteristics approach $R_{+}(\lambda)$ as $t \rightarrow-\infty$ by Proposition 6.8, the last part follows from $(H-\lambda) u_{+}=v$ and Theorem 10.9. It can be also proved by modifying the argument of Propositions 10.1-10.7 along the lines of our proof of Theorem 11.1. Namely, we consider the family $u_{t}^{+} \in \mathcal{C}^{-\infty}(X), t>0$, and note that for $t>0, R(\lambda+i t) \in \Psi_{\mathrm{Sc}}^{-2,0}(X, \mathcal{C})$, so $\mathrm{WF}_{\mathrm{Sc}}\left(u_{t}^{+}\right) \subset \mathrm{WF}_{\mathrm{Sc}}(v)$. Let $A_{0}$, etc., be defined as $A_{r}$ with $r=0$ where $A_{r}$ is given by (10.40) (i.e. we do not need to use the approximating factor $\left.(1+r / x)^{-1}\right)$. Then

$$
\left\langle u_{t}^{+}, i\left[A_{0}^{*} A_{0}, H\right] u_{t}^{+}\right\rangle=-2 \operatorname{Im}\left\langle u_{t}^{+}, A_{0}^{*} A_{0}(H-(\lambda+i t)) u_{t}^{+}\right\rangle-2 t\left\|A_{0} u_{t}^{+}\right\|^{2} .
$$

Note that the pairings make sense since now $\mathrm{WF}_{\mathrm{Sc}}^{\prime}\left(A_{0}\right)$ is disjoint from $\mathrm{WF}_{\mathrm{Sc}}\left(u_{t}^{+}\right), t>0$. Thus,

$$
\begin{aligned}
\left\|B_{0} u_{t}^{+}\right\|^{2}+2 t\left\|A_{0} u_{t}^{+}\right\|^{2} \leqslant & \left|\left\langle u_{t}^{+}, E_{0} u_{t}^{+}\right\rangle\right|+\left|\left\langle u_{t}^{+}, F_{0} u_{t}^{+}\right\rangle\right| \\
& +2\left|\left\langle u_{t}^{+}, A_{0}^{*} A_{0}(H-(\lambda+i t)) u_{t}^{+}\right\rangle\right| .
\end{aligned}
$$

Since $t>0$, the second term can be dropped from the left hand side. Thus, knowing that $u_{t}^{+} \rightarrow u_{+}$in $\mathcal{C}^{-\infty}(X)$ as $t \rightarrow 0$, and assuming that $\xi_{0} \notin \mathrm{WF}_{\mathrm{Sc}}^{m, l}\left(u_{+}\right)$has already been proved and (10.50) is satisfied by $u_{+}$, we conclude that $\xi_{0} \notin \mathrm{WF}_{\mathrm{Sc}}^{m, l+1 / 2}\left(u_{+}\right)$. The iteration of this argument of Proposition 10.1 and the similar arguments for tangential propagation allow us to conclude the forward propagation estimates which can then be turned into maximal statements as we did in Theorem 10.9. This argument also shows the influence of the sign of $t$ : if $t<0$, the inequality (11.18) cannot be used to derive results on $u_{+}$. Instead, the signs are then correct in the backward estimate, just as expected.

We conclude this section with the following uniqueness theorem on solutions of $(H-\lambda) u=0$. It is essentially a geometric version of Isozaki's uniqueness theorem [16, Theorem 1.3], though we allow arbitrary growth of $u$ away from one of the radial sets, say $R_{+}(\lambda)$.

THEOREM 11.4. - Suppose that $H$ is a many-body Hamiltonian satisfying (10.1), $\lambda>0$. Suppose also that $u \in \mathcal{C}^{-\infty}(X),(H-\lambda) u=0$ and $\mathrm{WF}_{\mathrm{Sc}}^{m, l}(u) \cap R_{+}(\lambda)=\emptyset$ for some $m$ and some $l>-1 / 2$. Then $u=0$. The same conclusion holds if we replace $R_{+}(\lambda)$ by $R_{-}(\lambda)$.

Proof. - Just as in the proof of Theorem 11.3, the positive commutator estimate of Theorem 11.1 (but now applied with a regularizing factor in $x$ ) shows that $\mathrm{WF}_{\mathrm{Sc}}(u) \cap R_{+}(\lambda)=\emptyset$, 
and then Theorem 10.9 shows that

$$
\mathrm{WF}_{\mathrm{Sc}}(u) \subset R_{-}(\lambda) .
$$

We remark that although we need a regularizing factor here which requires some changes in the proof, e.g. see the argument of the paragraph below, the regularizing factor (whether $(1+r / x)^{-1}$ or another one) commutes with $V$, so the additional arguments for dealing with it are essentially the same as the two-body ones. Thus, the regularization part of the proof of $\mathrm{WF}_{\mathrm{Sc}}(u) \cap R_{+}(\lambda)=\emptyset$ essentially follows [22, Proposition 10].

We proceed to show that

$$
m \in \mathbb{R}, \quad l<-1 / 2 \Rightarrow \mathrm{WF}_{\mathrm{Sc}}^{m, l}(u) \cap R_{-}(\lambda)=\emptyset .
$$

We give the details below since regularity arguments for distributions which are large at infinity seem to appear less often in the literature than the 'finer ones'; in particular, [16, Theorem 1.3] assumes $u \in H_{\mathrm{sc}}^{m, l}(X)$ with $l>-1$. We essentially follow the proof of [22, Proposition 9] below.

So suppose that (11.20) has been shown for some $l<-1$; we now show it with $l$ replaced by $l+1 / 2$. This time we consider

$$
q=x^{-l-1} \chi(\tau) \tilde{\psi}(x), \quad l<-1,
$$

where $\tilde{\psi} \in \mathcal{C}_{c}^{\infty}(\mathbb{R})$ is identically 1 near 0 and is supported in a bigger neighborhood of 0 (it is simply a cutoff near $\partial X), \chi \in \mathcal{C}_{c}^{\infty}(\mathbb{R} ;[0,1])$ identically 1 on $(-\infty,-\sqrt{\lambda}+\varepsilon)$, vanishes on $(-\sqrt{\lambda}+2 \varepsilon, \infty), \varepsilon>0$, and $\chi$ vanishes with all derivatives at every $t$ with $\chi(t)=0$. Then

$$
\begin{gathered}
{ }^{\mathrm{sc}} H_{g} q=-2\left((l+1) \tau \chi(\tau)+h \chi^{\prime}(\tau)\right) x^{-l-1}=\left(-b^{2}+e\right) x^{-l-1}, \\
b^{2}=2(l+1) \tau \chi(\tau) .
\end{gathered}
$$

The first key point now is that on $\mathrm{WF}_{\mathrm{Sc}}(u)$ we have $\tau=-\sqrt{\lambda}$, so $\mathrm{WF}_{\mathrm{Sc}}(u) \cap \pi(\operatorname{supp} e)=\emptyset$. Let $A \in \Psi_{\mathrm{Sc}}^{-\infty,-l-1}(X, \mathcal{C})$ as in Lemma 9.1. Corollary 9.7 again shows that for $\psi \in \mathcal{C}_{c}^{\infty}(\mathbb{R} ;[0,1])$ supported sufficiently close to $\lambda$ we have

$$
i x^{l+1 / 2} \psi(H)\left[A^{*} A, H\right] \psi(H) x^{l+1 / 2} \geqslant x^{l+1 / 2}\left(\left(2-\varepsilon^{\prime}\right) B^{*} B+E+F\right) x^{l+1 / 2},
$$

where

$$
\varepsilon^{\prime}>0
$$

$$
\begin{aligned}
& B \in \Psi_{\mathrm{Sc}}^{-\infty,-l-1 / 2}(X, \mathcal{C}), \quad \hat{B}_{a,-l-1 / 2}(\zeta)=b(\zeta) q(\zeta)^{1 / 2} \psi\left(\hat{H}_{a, 0}(\zeta)\right), \\
& E \in \Psi_{\mathrm{Sc}}^{-\infty,-2 l-1}(X, \mathcal{C}), \quad \mathrm{WF}_{\mathrm{Sc}}^{\prime}(E) \cap \mathrm{WF}_{\mathrm{Sc}}(u)=\emptyset \\
& F \in \Psi_{\mathrm{Sc}}^{-\infty,-2 l}(X, \mathcal{C}), \quad \mathrm{WF}_{\mathrm{Sc}}^{\prime}(F) \subset \operatorname{supp}\left(x^{l+1} q\right) .
\end{aligned}
$$

Let

$$
\begin{aligned}
& A_{r}=A(1+r / x)^{-1} \psi(H), \quad B_{r}=B(1+r / x)^{-1}, \\
& E_{r}=(1+r / x)^{-1} E(1+r / x)^{-1},
\end{aligned}
$$

so

(11.27) $A_{r} \in \Psi_{\mathrm{Sc}}^{-\infty,-l}(X, \mathcal{C})$ for $r>0, A_{r}$ is uniformly bounded in $\Psi_{\mathrm{Sc}}^{-\infty,-l-1}(X, \mathcal{C})$; 
analogous statements also hold for $B_{r}$ and $E_{r}$. Thus,

$$
i x^{l+1 / 2}\left[A_{r}^{*} A_{r}, H\right] x^{l+1 / 2}=i(1+r / x)^{-1} x^{l+1 / 2} \psi(H)\left[A^{*} A, H\right] \psi(H) x^{l+1 / 2}(1+r / x)^{-1}
$$

$$
+i \psi(H) A^{*} x^{l+1}\left(G_{r}+G_{r}^{*}\right) x^{l+1} A \psi(H)+H_{r}
$$

where $H_{r}$ is uniformly bounded in $\Psi_{\mathrm{Sc}}^{-\infty, 1}(X, \mathcal{C})$ and

$$
G_{r}=i \psi_{0}(H)^{2} x^{-1}(1+r / x)^{-1}\left[(1+r / x)^{-1}, H\right],
$$

$\psi_{0} \in \mathcal{C}_{c}^{\infty}(\mathbb{R} ;[0,1]), \psi_{0} \equiv 1$ on $\operatorname{supp} \psi$, so $G_{r}$ is uniformly bounded in $\Psi_{\mathrm{Sc}}^{-\infty, 1}(X, \mathcal{C})$. Thus, we need to estimate the commutator $\left[(1+r / x)^{-1}, H\right]$, and now we do not have a large $M$ as in the proof of Proposition 10.1 to help us deal with it.

The other key point is thus that we have $i\left[(1+r / x)^{-1}, H\right]=i\left[(1+r / x)^{-1}, \Delta\right]$ and

$$
{ }^{\mathrm{sc}} H_{g}(1+r / x)^{-1}=2 \tau \frac{r}{x+r}=-c_{r}^{2}+d_{r}, \quad c_{r}=\chi_{1}(\tau)(-\tau)^{1 / 2}\left(\frac{r}{x+r}\right)^{1 / 2}
$$

$\chi_{1} \in \mathcal{C}_{c}^{\infty}(\mathbb{R} ;[0,1])$ identically 1 on $(-\infty,-\sqrt{\lambda}+3 \varepsilon)$, vanishes on $(-\sqrt{\lambda}+4 \varepsilon, \infty), \varepsilon>0$. Let $C_{r}$ be the quantization of $c_{r}$ multiplied by $\psi_{0}(H)$ as in Lemma 9.1, and define $D_{r}$ similarly but with $\psi_{0}(H)$ replaced by $\psi_{0}(H)^{2}$. Thus, as $(1+r / x)^{-1}$ is uniformly bounded in the symbol class $S^{0}(X)$,

$$
i \psi_{0}(H) x^{-1 / 2}\left[(1+r / x)^{-1}, H\right] x^{-1 / 2} \psi_{0}(H)=C_{r}^{*} C_{r}+D_{r}+H_{r}^{\prime}
$$

with $C_{r}$ and $D_{r}$ uniformly bounded in $\Psi_{\mathrm{Sc}}^{-\infty, 0}(X, \mathcal{C}), C_{r} \in \Psi_{\mathrm{Sc}}^{-\infty, 1 / 2}(X, \mathcal{C})$ for $r>0$, $D_{r} \in \Psi_{\mathrm{Sc}}^{-\infty, 1}(X, \mathcal{C})$ for $r>0$, and $H_{r}^{\prime}$ uniformly bounded in $\Psi_{\mathrm{sc}}^{-\infty, 1}(X, \mathcal{C})$. Moreover, $D_{r} A \in \Psi_{\mathrm{Sc}}^{-\infty, \infty}(X, \mathcal{C})$ uniformly due to the disjoint operator wave front sets. Thus,

$$
G_{r}+G_{r}^{*}=2(1+r / x)^{-1 / 2}\left(C_{r}^{*} C_{r}+D_{r}\right)(1+r / x)^{-1 / 2}+H_{r}^{\prime \prime}
$$

with $H_{r}^{\prime \prime}$ uniformly bounded in $\Psi_{\mathrm{Sc}}^{-\infty, 1}(X, \mathcal{C})$, so

$$
\text { (11.33) } \geqslant H_{r}^{\sharp} \text {, }
$$

$$
\begin{aligned}
& \psi(H) A^{*} x^{l+1}\left(G_{r}+G_{r}^{*}\right) x^{l+1} A \psi(H) \\
& \quad=2 \psi(H) A^{*} x^{l+1}(1+r / x)^{-1 / 2}\left(C_{r}^{*} C_{r}+D_{r}\right)(1+r / x)^{-1 / 2} x^{l+1} A \psi(H)+H_{r}^{b}
\end{aligned}
$$

$H_{r}^{b}, H_{r}^{\sharp}$ uniformly bounded in $\Psi_{\mathrm{Sc}}^{-\infty, 1}(X, \mathcal{C})$. Combining (11.24), (11.28) and (11.33), we see that for $\varepsilon^{\prime}>0$ we have

$$
i x^{l+1 / 2}\left[A_{r}^{*} A_{r}, H\right] x^{l+1 / 2} \geqslant x^{l+1 / 2}\left(\left(2-\varepsilon^{\prime}\right) B_{r}^{*} B_{r}+E_{r}+F_{r}\right) x^{l+1 / 2} .
$$

We deduce as at the end of the proof of Proposition 10.1 that $\mathrm{WF}_{\mathrm{Sc}}^{m, l+1 / 2}(u) \cap R_{-}(\lambda)=\emptyset$ for every $m$ and for every $l+1 / 2<-1 / 2$, so (11.20) holds. In particular, $u \in H_{\mathrm{sc}}^{m, l}(X)$ for every $m$ and for every $l<-1 / 2$.

In the Euclidean setting we can now simply refer to Isozaki's uniqueness theorem [16, Theorem 1.3] to conclude that $u=0$. Here we give some details to indicate how this conclusion can be reached in general. The crucial step is improving the estimate past the critical regularity $H_{\mathrm{sc}}^{*,-1 / 2}(X)$. In the Euclidean setting this was done by Isozaki [15, Lemma 4.5] and his 
argument was adapted to the geometric setting in [40, Proposition 17.8]. We thus conclude that $\mathrm{WF}_{\mathrm{Sc}}^{m, l}(u) \cap R_{-}(\lambda)=\emptyset$ for $l \in(0,-1 / 2)$. This is the point where $(H-\lambda) u=0$, and not just $(H-\lambda) u \in \dot{\mathcal{C}}^{\infty}(X)$ is used. Now we can apply a commutator estimate like that of Theorem 11.1 but near $R_{-}(\lambda)$. Thus, we conclude that $\mathrm{WF}_{\mathrm{Sc}}(u) \cap R_{-}(\lambda)=\emptyset$, so $u \in \dot{\mathcal{C}}^{\infty}(X)$. The theorem of Froese and Herbst [4] on the absence of bound states with positive energy adapted to the geometric setting, as discussed in [40, Appendix B], concludes that $u=0$.

\section{The Poisson operator and the scattering matrix}

Just as in $[36,40]$ where three-body scattering was analyzed, the propagation of singularities for generalized eigenfunctions of $H$ implies the corresponding result for the (free-to-free) scattering matrix, $S(\lambda)$, of $H$. Note that this is the only S-matrix under our assumption of the absence of bound states of the subsystems. We start by discussing the Poisson operator, then we use it to relate the propagation of singularities for generalized eigenfunctions to the wave front relation of the S-matrix.

The result that allows us to define the Poisson operator is that if $V$ is short-range, i.e. $V \in x^{2} \mathcal{C}^{\infty}\left(X \backslash C_{0, \text { sing }}\right)$, then for $\lambda \in(0, \infty)$ and $g \in \mathcal{C}_{c}^{\infty}\left(C_{0}^{\prime}\right)$, there is a unique $u \in \mathcal{C}^{-\infty}(X)$ such that $(H-\lambda) u=0$, and $u$ has the form

$$
u=\mathrm{e}^{-i \sqrt{\lambda} / x} x^{(n-1) / 2} v_{-}+R(\lambda+i 0) f
$$

where $v_{-} \in \mathcal{C}^{\infty}(X),\left.v_{-}\right|_{\partial X}=g$, and $f \in \dot{\mathcal{C}}^{\infty}(X)$. For long-range $V$ the same statement is valid with the asymptotic expansion replaced by one similar to that of Theorem 11.1:

$$
u=\mathrm{e}^{-i \sqrt{\lambda} / x} x^{(n-1) / 2+i \alpha_{-}} v_{-}+R(\lambda+i 0) f, \quad v_{-} \in \mathcal{A}_{\mathrm{phg}}^{\mathcal{K}}(X) .
$$

The Poisson operator with initial state in the free-cluster is then the map

$$
P_{+}(\lambda): \mathcal{C}_{c}^{\infty}\left(C_{0}^{\prime}\right) \rightarrow \mathcal{C}^{-\infty}\left(\mathbb{S}_{+}^{n}\right), \quad P_{+}(\lambda) g=u
$$

(Note that the subscript 0 for the free cluster has been dropped here in contrast to the introduction and [39].) To see that such a $u$ is unique, note that the difference $v=u-u^{\prime}$ of two distributions $u$ and $u^{\prime}$ with the above properties satisfies $(H-\lambda) v=0$ and $\operatorname{WF}_{\mathrm{Sc}}^{0,0}(v) \cap R_{+}(\lambda)=\emptyset$ by Theorem 11.1, so $v=0$ due to Theorem 11.4. To see the existence of such $u$, note that as supp $g \subset C_{0}^{\prime}$, we can construct

$$
\begin{aligned}
& u_{-}=\mathrm{e}^{-i \sqrt{\lambda} / x} x^{(n-1) / 2} v_{-}, \quad v_{-} \in \mathcal{C}^{\infty}(X),\left.\quad v_{-}\right|_{\partial X}=g, \\
& -f=(H-\lambda) u_{-} \in \dot{\mathcal{C}}^{\infty}(X),
\end{aligned}
$$

by a local calculation as in [22], i.e. essentially in a two-body type setting. (We need to make slight changes in the asymptotic expansion for long-range $V$ as described above.) Thus, we construct the Taylor series of $v_{-}$at $\partial X$ explicitly, so we can even arrange that $\operatorname{supp} v_{-} \cap$ $C_{0, \text { sing }}=\emptyset$. Then $u=u_{-}+R(\lambda+i 0) f$ is of the form (12.1) and satisfies $(H-\lambda) u=0$ indeed.

We need to understand the Poisson operator better before we can extend it to distributions. So first recall from [40, Section 19] that the Melrose-Zworski [25] construction of a parametrix for the Poisson operator in the two-body type setting ( $\mathcal{C}$ is empty) gives 'the initial part' of a parametrix $\tilde{P}_{+}(\lambda)$ for the Poisson operator with free initial state in the many-body setting (for three bodies in that paper, but this makes no difference). Although the construction is performed 
there for short range potentials, it can be easily adjusted to long range potentials decaying like $x$, see [40, Appendix A] and [38, Section 3]. In particular, the kernel of $\tilde{P}_{ \pm}(\lambda)$ is of the form

$$
K_{ \pm}^{b}\left(x, y, y^{\prime}\right)=\mathrm{e}^{\mp i \sqrt{\lambda} \cos \operatorname{dist}\left(y, y^{\prime}\right) / x} x^{i \alpha_{\mp}\left(y^{\prime}\right)} a_{ \pm}\left(x, y, y^{\prime}\right)|d h|,
$$

where dist is the distance function of the boundary metric $h,|d h|$ is the Riemannian density associated with it, $\alpha_{ \pm}$are given by (1.20), and $a_{ \pm} \in \mathcal{C}^{\infty}\left(X \times C_{0}^{\prime}\right)$ are cut off to be supported near $y=y^{\prime}$. Here $y^{\prime}$ is the 'initial point' of the plane waves, so $y^{\prime} \in C_{0}^{\prime}$ corresponds to considering free incoming particles. In Euclidean scattering $K_{ \pm}^{b}$ takes the form $\mathrm{e}^{\mp i \sqrt{\lambda} w \cdot y^{\prime}} a_{ \pm}\left(w, y^{\prime}\right)|d h|, w=y / x$ is the Euclidean variable and $|d h|$ the standard measure on the sphere; and e.g. if the potentials $V_{b}$ are Schwartz then $a_{ \pm}$are just cutoff functions supported near $y=y^{\prime}$ which are constant in a smaller neighborhood of $y=y^{\prime}$. In general, $a_{ \pm}(0, y, y)$ is determined by the condition that

$$
\tilde{P}_{ \pm}(\lambda) g=\mathrm{e}^{\mp i \sqrt{\lambda} / x} x^{i \alpha_{\mp}+(n-1) / 2} v_{\mp},
$$

$v_{\mp} \in \mathcal{A}_{\mathrm{phg}}^{\mathcal{K}}(X),\left.v_{\mp}\right|_{\partial X}=g$, and then $a_{ \pm}\left(0, y, y^{\prime}\right)$, as well as the other terms of the Taylor series of $a_{ \pm}$at $x=0$ can be calculated from transport equations near $y=y^{\prime}$. Finally, we cut off the solutions to the transport equations close to $y=y^{\prime}$ before reaching $C_{0, \text { sing }}$; we do this by multiplying the asymptotically summed solutions of the transport equations by a function $\phi \in \mathcal{C}^{\infty}\left(X \times C_{0}^{\prime}\right)$ which is identically 1 near diagonal of $C_{0} \times C_{0}^{\prime}$. (Note that such a function $\phi$ is not smooth on $X \times C_{0}$.) This defines the kernel $K_{ \pm}^{b}$ as a distribution in $\mathcal{C}^{-\infty}\left(X \times C_{0}^{\prime}\right)$. Thus, for $y^{\prime}$ in a fixed compact subset $K_{0}$ in $C_{0}^{\prime}, K_{ \pm}^{b}\left(x, y, y^{\prime}\right)$ is supported away from $C_{0 \text {,sing }}$, so for $g \in \mathcal{C}_{c}^{-\infty}\left(C_{0}^{\prime}\right), \operatorname{supp}\left(\tilde{P}_{ \pm}(\lambda) g\right)$ is disjoint from $C_{0, \text { sing }}$.

The most important properties of $\tilde{P}_{ \pm}(\lambda)$ are summarized in the following proposition. Although we state them for $\tilde{P}_{+}(\lambda)$ only, they also hold for $\tilde{P}_{-}(\lambda)$ with the appropriate sign changes. Here we use $\sim_{ \pm}^{\prime}$ as the relation on $S^{*} \partial X \times \Sigma_{\Delta-\lambda}$ defined analogously to $\sim_{ \pm}$(see Definition 6.9), but with 'generalized broken bicharacteristics' replaced by 'bicharacteristics of $\Delta-\lambda$ '. Note that generalized broken bicharacteristics are simply bicharacteristics in ${ }^{\mathrm{sc}} T_{C_{0}^{\prime}}^{*} X$ which is where we will apply to following result.

Proposition 12.1 ([40, Proposition A.1]). $-K_{+}^{b} \in \mathcal{C}^{-\infty}\left(X \times C_{0}^{\prime} ; \Omega_{R}\right)$, constructed above, is the kernel of an operator $\tilde{P}_{+}(\lambda): \mathcal{C}_{c}^{\infty}\left(C_{0}^{\prime}\right) \rightarrow \mathcal{C}^{-\infty}(X)$, which extends to an operator $\tilde{P}_{+}(\lambda): \mathcal{C}_{c}^{-\infty}\left(C_{0}^{\prime}\right) \rightarrow \mathcal{C}^{-\infty}(X)$, and for $g \in \mathcal{C}_{c}^{-\infty}\left(C_{0}^{\prime}\right)$

$$
\operatorname{supp}\left(\tilde{P}_{+}(\lambda) g\right) \cap C_{0, \text { sing }}=\emptyset,
$$

$$
\begin{aligned}
& \mathrm{WF}_{\mathrm{sc}}\left(\tilde{P}_{+}(\lambda) g\right) \subset\{\{(y, \sqrt{\lambda}, 0): y \in \operatorname{supp} g\} \\
& \cup\left\{\xi \in \dot{\Sigma} \backslash\left(R_{+}(\lambda) \cup R_{-}(\lambda)\right): \exists \zeta \in \mathrm{WF}(g), \xi \sim_{-}^{\prime} \zeta\right\}, \\
& \mathrm{WF}_{\mathrm{sc}}\left((H-\lambda) \tilde{P}_{+}(\lambda) g\right) \subset\left\{\xi \in \dot{\Sigma} \backslash\left(R_{+}(\lambda) \cup R_{-}(\lambda)\right): \exists \zeta \in \mathrm{WF}(g), \xi \sim_{-}^{\prime} \zeta\right\} .
\end{aligned}
$$

The actual Poisson operator is then given by

$$
P_{+}(\lambda)=\tilde{P}_{+}(\lambda)-R(\lambda+i 0)(H-\lambda) \tilde{P}_{+}(\lambda),
$$

with a similar definition of $P_{-}(\lambda)$ :

$$
P_{-}(\lambda)=\tilde{P}_{-}(\lambda)-R(\lambda-i 0)(H-\lambda) \tilde{P}_{-}(\lambda) .
$$


Indeed, if $g \in \mathcal{C}_{c}^{\infty}\left(C_{0}^{\prime}\right)$ then $(H-\lambda) \tilde{P}_{+}(\lambda) g \in \dot{\mathcal{C}}^{\infty}(X)$ and $\tilde{P}_{+}(\lambda) g$ has an asymptotic expansion as in (12.6), so by Theorem 11.1, $(H-\lambda) P_{+}(\lambda) g=0$ and $P(\lambda) g$ has the form (12.1) (with changes as indicated in (12.2) if $V$ is long-range). In addition, for $g \in \mathcal{C}_{c}^{-\infty}\left(C_{0}^{\prime}\right), \mathrm{WF}_{\mathrm{Sc}}((H-$

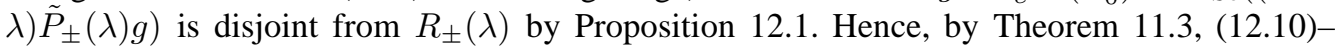
(12.11) indeed make sense. We also immediately deduce from Theorem 11.3

Proposition 12.2.- Suppose that $H$ is a many-body Hamiltonian satisfying (10.1). Then the Poisson operator $P_{+}(\lambda): \mathcal{C}_{c}^{\infty}\left(C_{0}^{\prime}\right) \rightarrow \mathcal{C}^{-\infty}(X)$ extends by continuity to an operator $\tilde{P}_{+}(\lambda): \mathcal{C}_{c}^{-\infty}\left(C_{0}^{\prime}\right) \rightarrow \mathcal{C}^{-\infty}(X)$. Moreover, for $g \in \mathcal{C}_{c}^{-\infty}\left(C_{0}^{\prime}\right)$ we have

$$
\begin{aligned}
\mathrm{WF}_{\mathrm{Sc}}\left(P_{+}(\lambda) g\right) \subset & \{(y, \sqrt{\lambda}, 0): y \in \operatorname{supp} g\} \cup R_{-}(\lambda) \\
& \cup\left\{\xi \in \dot{\Sigma}(\lambda) \backslash R_{+}(\lambda): \exists \zeta \in \mathrm{WF}(g), \xi \sim_{-} \zeta\right\} .
\end{aligned}
$$

Our definition of the free-to-free S-matrix is based on asymptotic expansions of generalized eigenfunctions. So let $g \in \mathcal{C}_{c}^{\infty}\left(C_{0}^{\prime}\right)$ and let $u=P_{+}(\lambda) g$. By (12.1) (modified as in (12.2) for long-range $V$ ) and Theorem 11.1, $u$ has the form

$$
u=\mathrm{e}^{-i \sqrt{\lambda} / x} x^{(n-1) / 2} v_{-}+\mathrm{e}^{i \sqrt{\lambda} / x} x^{(n-1) / 2} v_{+}
$$

with $v_{-} \in \mathcal{C}^{\infty}(X), v_{+} \in \mathcal{C}^{\infty}\left(X \backslash C_{0, \text { sing }}\right),\left.v_{-}\right|_{\partial X}=g$. We then define the free-to-free S-matrix by

$$
S(\lambda): \mathcal{C}_{c}^{\infty}\left(C_{0}^{\prime}\right) \rightarrow \mathcal{C}^{\infty}\left(C_{0}^{\prime}\right), \quad S(\lambda) g=\left.v_{+}\right|_{C_{0}^{\prime}} .
$$

We need a better description of the S-matrix to describe its structure. This can be done via a boundary pairing formula analogous to [22, Proposition 13]. It gives the following alternative description of the S-matrix, see [39, Equation (5.7)] (or its analogue from [36] in the nonEuclidean setting):

Proposition 12.3. - For $\lambda>0$ the scattering matrix is given by

$$
S(\lambda)=\frac{1}{2 i \sqrt{\lambda}}\left((H-\lambda) \tilde{P}_{-}(\lambda)\right)^{*} P_{+}(\lambda) .
$$

Here $\left((H-\lambda) \tilde{P}_{-}(\lambda)\right)^{*}$ denotes the formal adjoint, i.e. the meaning of $(12.15)$ is that for all $f, g \in \mathcal{C}_{c}^{\infty}\left(C_{0}^{\prime}\right)$

$$
\langle f, S(\lambda) g\rangle_{\partial X}=\left\langle(H-\lambda) \tilde{P}_{-}(\lambda) f, \frac{1}{2 i \sqrt{\lambda}} P_{+}(\lambda) g\right\rangle_{X} .
$$

Proof. - The following pairing formula was proved by Melrose [22, Proposition 13] for shortrange $V$, but the same proof also applies when $V$ is long-range. Also, the proof can be easily localized, see [39, Proposition 3.3]. Suppose that for $j=1,2, u_{j} \in \mathcal{C}^{-\infty}(X)$,

$$
\begin{aligned}
& u_{j}=\mathrm{e}^{i \sqrt{\lambda} / x} x^{(n-1) / 2+i \alpha_{+}} v_{j,+}+\mathrm{e}^{-i \sqrt{\lambda} / x} x^{(n-1) / 2+i \alpha_{-}} v_{j,-}, \\
& v_{j, \pm} \in \mathcal{A}_{\text {phg }}^{\mathcal{K}}\left(X \backslash C_{0, \text { sing }}\right), \quad \operatorname{supp}\left(v_{2, \pm}\right) \Subset X \backslash C_{0, \text { sing }},
\end{aligned}
$$

and $f_{j}=(H-\lambda) u_{j} \in \dot{\mathcal{C}}^{\infty}(X)$. Let $a_{j, \pm}=\left.v_{j, \pm}\right|_{\partial X}$. Then

$$
2 i \sqrt{\lambda} \int_{\partial X}\left(a_{1,+} \overline{a_{2,+}}-a_{1,-} \overline{a_{2,-}}\right) d h=\int_{X}\left(u_{1} \overline{f_{2}}-f_{1} \overline{u_{2}}\right) d g .
$$


We apply this result with $u_{1}=P_{+}(\lambda) g, u_{2}=\tilde{P}_{-}(\lambda) f, f, g \in \mathcal{C}_{c}^{\infty}\left(C_{0}^{\prime}\right)$. By the construction of $\tilde{P}(-\lambda)$ we conclude that $a_{2,+}=f, a_{2,-}=0$, while for $u_{1}$ we see directly from the definition of $S(\lambda)$ and $P(\lambda)$ that $a_{1,-}=g, a_{1,+}=S(\lambda) g$. Substitution into (12.18) proves the proposition.

Propositions 12.1 and 12.2, when combined with (12.15), allow us to deduce the structure of the S-matrix.

THEOREM 12.4. - Let $(X, \mathcal{C})$ be a locally linearizable many-body space. Suppose that $H$ is a many-body Hamiltonian satisfying (10.1). Then the scattering matrix, $S(\lambda)$, extends to a continuous linear map $\mathcal{C}_{c}^{-\infty}\left(C_{0}^{\prime}\right) \rightarrow \mathcal{C}^{-\infty}\left(C_{0}^{\prime}\right)$. The wave front relation of $S(\lambda)$ is given by the generalized broken geodesic flow at time $\pi$.

Proof. - Let $f, g \in \mathcal{C}_{c}^{-\infty}\left(C_{0}^{\prime}\right)$. Suppose also that there is no generalized broken geodesic of length $\pi$ starting at some $\zeta \in \mathrm{WF}(g)$ and ending at $\zeta^{\prime} \in \mathrm{WF}(f)$. That means that for any $\xi \in \dot{\Sigma} \backslash\left(R_{+}(\lambda) \cup R_{-}(\lambda)\right)$ we cannot have $\xi \sim_{-} \zeta, \zeta \in \mathrm{WF}(g)$, and $\xi \sim_{+} \zeta^{\prime}, \zeta^{\prime} \in \mathrm{WF}(f)$, at the same time. Proposition 12.1 (with - signs instead of + ) implies that

$$
\mathrm{WF}_{\mathrm{Sc}}\left((H-\lambda) \tilde{P}_{-}(\lambda) f\right) \subset \dot{\Sigma} \backslash\left(R_{+}(\lambda) \cup R_{-}(\lambda)\right) ;
$$

indeed, we also have $\mathrm{WF}_{\mathrm{Sc}}\left((H-\lambda) \tilde{P}_{-}(\lambda) f\right) \subset{ }^{\mathrm{sc}} T_{C_{0}^{\prime}}^{*} X$, so we can even replace $\mathrm{WF}_{\mathrm{Sc}}$ by $\mathrm{WF}_{\mathrm{sc}}$. Thus, by our assumption on $\mathrm{WF}(f)$ and $\mathrm{WF}(g)$, and by Propositions 12.1-12.2, we have

$$
\mathrm{WF}_{\mathrm{sc}}\left((H-\lambda) \tilde{P}_{-}(\lambda) f\right) \cap \mathrm{WF}_{\mathrm{sc}}\left(P_{+}(\lambda) g\right)=\emptyset .
$$

But the complex pairing

$$
\left\langle u, u^{\prime}\right\rangle_{X}=\int u \overline{u^{\prime}} d g
$$

extends by continuity from $u, u^{\prime} \in \dot{\mathcal{C}}^{\infty}(X)$ to $u, u^{\prime} \in \mathcal{C}^{-\infty}(X)$ satisfying $\mathrm{WF}_{\mathrm{sc}}(u) \cap \mathrm{WF}_{\mathrm{sc}}\left(u^{\prime}\right)$ $=\emptyset$. To see this just let $A \in \Psi_{\mathrm{sc}}^{0,0}(X)$ with $\mathrm{WF}_{\mathrm{sc}}^{\prime}(A) \cap \mathrm{WF}_{\mathrm{sc}}(u)=\emptyset$, $\mathrm{WF}_{\mathrm{sc}}^{\prime}\left(\operatorname{Id}-A^{*}\right) \cap$ $\mathrm{WF}_{\mathrm{sc}}\left(u^{\prime}\right)=\emptyset$, and note that

$$
\left\langle u, u^{\prime}\right\rangle_{X}=\left\langle A u, u^{\prime}\right\rangle_{X}+\left\langle u,\left(\operatorname{Id}-A^{*}\right) u^{\prime}\right\rangle_{X}
$$

extends as claimed. Hence, the pairing

$$
\left\langle P_{+}(\lambda) g,(H-\lambda) \tilde{P}_{-}(\lambda) f\right\rangle_{X}=\left\langle\left((H-\lambda) \tilde{P}_{-}(\lambda)\right)^{*} P_{+}(\lambda) g, f\right\rangle_{X}
$$

defined first for $f, g \in \mathcal{C}_{c}^{\infty}\left(C_{0}^{\prime}\right)$ extends by continuity to $f, g \in \mathcal{C}_{c}^{-\infty}\left(C_{0}^{\prime}\right)$ satisfying our wave front condition. In other words, $g$ can be paired with every distribution whose wave front set has no elements related to $\mathrm{WF}(g)$ by the generalized broken geodesic flow at time $\pi$. Thus, for any $A \in \Psi_{c}^{0}\left(C_{0}^{\prime}\right)$ with $\mathrm{WF}^{\prime}(A)$ disjoint from the image of $\mathrm{WF}(g)$ under the generalized broken geodesic flow at time $\pi$, and for any $f \in \mathcal{C}_{c}^{-\infty}\left(C_{0}^{\prime}\right),\langle A S(\lambda) g, f\rangle_{\partial X}=\left\langle S(\lambda) g, A^{*} f\right\rangle_{\partial X}$ is defined by continuity from $f \in \mathcal{C}_{c}^{\infty}\left(C_{0}^{\prime}\right)$, so $A S(\lambda) g \in \mathcal{C}^{\infty}\left(C_{0}^{\prime}\right)$. But this states exactly that $\mathrm{WF}(S(\lambda) g)$ is contained in the image of $\mathrm{WF}(g)$ under the generalized broken geodesic flow at time $\pi$.

\section{Appendix A. The proof of Proposition 6.3}

In this appendix we prove Proposition 6.3 under the assumption that $\mathcal{C}$ is totally geodesic, roughly following Lebeau's original proof in [18]. As noted after the statement of the proposition 
we can proceed inductively, using the order on $\mathcal{C}$. So assume that $\gamma\left(t_{0}\right)=\xi_{0} \in \Sigma_{n}(\lambda) \cap$ ${ }^{\mathrm{sc}} T_{C_{a}^{\prime}}^{*}\left(C_{a} ; X\right)$. The inductive hypothesis is that we have already proved the proposition for $b$ with $C_{a} \subset C_{b}$. Thus, by Definition 6.2, part (ii), there exists $\delta^{\prime}>0$ such that the conclusion of the proposition holds if we replace $t_{0}$ by $t \neq t_{0}$, assuming $\left|t-t_{0}\right|<\delta^{\prime}$. Let $\tilde{\xi}_{ \pm}(t) \in \Sigma_{\Delta-\lambda}$, $t \neq t_{0}$, be the points given by the inductive hypothesis. We often write

$$
\tilde{\xi}_{ \pm}(t)=\left(y(t), z(t), \tau(t), \mu_{ \pm}(y), \nu(t)\right)
$$

in local coordinates, so e.g. $\tau\left(\tilde{\xi}_{ \pm}(t)\right)=\tau(t)$. Note that $\pi\left(\tilde{\xi}_{ \pm}(t)\right)=\gamma(t)$, hence the independence of the $\pi$-invariant coordinates, $y, z, \tau$ and $\nu$, of the \pm signs.

Notice first that $\tau$ is $\pi$-invariant, so for $t \neq t_{0}$ we have

$$
\begin{aligned}
d(\tau \circ \gamma) /\left.d t\right|_{t \pm} & ={ }^{\mathrm{sc}} H_{g} \tau\left(\tilde{\xi}_{ \pm}(t)\right)=-2 h\left(\tilde{\xi}_{ \pm}(t)\right)=2\left(\tau\left(\tilde{\xi}_{ \pm}(t)\right)^{2}-\lambda\right) \\
& =2\left(\tau(\gamma(t))^{2}-\lambda\right)
\end{aligned}
$$

where we used that $\tau^{2}+h=\lambda$ in $\Sigma_{\Delta-\lambda}$. Thus, $\tau(t)=\tau(\gamma(t))$ is differentiable on $\left(t_{0}-\delta^{\prime}, t_{0}+\delta^{\prime}\right)$ except possibly at $t_{0}$, it is continuous at $t_{0}$, and its derivative $\tau^{\prime}(t)$ extends to a continuous function on $\left(t_{0}-\delta^{\prime}, t_{0}+\delta^{\prime}\right)$. Hence $\tau(t)$ is differentiable at $t_{0}$ and $\tau^{\prime}\left(t_{0}\right)=2\left(\tau\left(t_{0}\right)^{2}-\lambda\right)=$ ${ }^{\mathrm{sc}} H_{g} \tau\left(\tilde{\xi}_{0}\right)$ for any $\tilde{\xi}_{0} \in \Sigma_{\Delta-\lambda}$. Notice also that, with the notation of (10.54) in the proof of Proposition 10.1, $\tau^{\prime}\left(t_{0}\right)=W_{0} \tau=\left({ }^{\mathrm{sc}} H_{g} \tau\right)\left(\tilde{\xi}_{0}\right)$. In particular,

$$
\left|\tau(t)-\tau_{0}\right| \leqslant C_{1}\left|t-t_{0}\right| \quad \text { if }\left|t-t_{0}\right|<\delta^{\prime} .
$$

In fact, the ODE $\tau^{\prime}(t)=2\left(\tau(t)^{2}-\lambda\right)$, satisfied for $\left|t-t_{0}\right|<\delta^{\prime}$, has a unique $\mathcal{C}^{\infty}$ solution, so on $\left(t_{0}-\delta^{\prime}, t_{0}+\delta^{\prime}\right), \tau(t)$ is $\mathcal{C}^{\infty}$ and

$$
\left|\tau(t)-\left(\tau_{0}+\left(W_{0} \tau\right)\left(t-t_{0}\right)\right)\right| \leqslant C\left|t-t_{0}\right|^{2} .
$$

From now on we only consider differentiability issues from the left at $t_{0}$; of course, the situation on the right is similar. We define the $\pi$-invariant functions $\eta=y \cdot \mu, \omega_{0}, \omega$ and $\phi=\phi^{(\varepsilon, \delta)}$ as in the proof of Proposition 10.1. It is shown there that there exist $C_{0}>0$ and $\delta_{0}>0$ such that if $\varepsilon \in(0,1), \delta \in\left(0, \delta_{0}\right), \delta<C_{0} \varepsilon^{2}$ and $\tilde{\xi}=(y, z, \tau, \mu, \nu) \in \Sigma_{\Delta-\lambda}$ satisfies $\tau_{0}-\tau \geqslant-2 \delta$ and $\phi(\tilde{\xi}) \leqslant 2 \delta$ then ${ }^{\mathrm{sc}} H_{g} \phi \geqslant c_{0}>0$. So suppose that we fixed some

$$
0<T<\min \left(\delta^{\prime}, C_{1} \delta_{0}\right)
$$

and let

$$
\delta=C_{1} T, \quad \varepsilon=2\left(\delta / C_{0}\right)^{1 / 2} .
$$

Thus, for $t \in\left[t_{0}-T, t_{0}\right),\left|\tau(t)-\tau_{0}\right|<2 \delta$. As $\phi$ is a $\pi$-invariant function which vanishes at $\xi_{0}$, we see that $F=\phi_{\pi} \circ \gamma$ satisfies $F(t)<0$ and $d F /\left.d t\right|_{t \pm}={ }^{\mathrm{sc}} H_{g} \phi\left(\tilde{\xi}_{ \pm}(t)\right) \geqslant c_{0}>0$ for $t \in\left[t_{0}-T, t_{0}\right)$ (cf. the proof of Proposition 7.1 after (7.35)). Taking into account the form of $\phi$ and (A.3), we deduce that for $t \in\left[t_{0}-T, t_{0}\right), \omega(t)=\omega(\gamma(t))$ satisfies

$$
\omega(t) \leqslant C_{1} \varepsilon^{4} \delta^{3}\left|t-t_{0}\right| .
$$

Applying this with $t=t_{0}-T$ we see that

$$
\omega\left(t_{0}-T\right) \leqslant C_{2} T^{6}
$$


Since $\omega$ is independent of $\varepsilon$ and $\delta$, we have deduced that there exists $\delta_{1}>0$ such that

$$
t_{0}-\delta_{1}<t<t_{0} \Rightarrow \omega(t) \leqslant C\left|t-t_{0}\right|^{6} .
$$

In particular, under the same assumption,

$$
\omega_{0}(t) \leqslant C^{\prime}\left|t-t_{0}\right|^{3}
$$

so

$$
\left|\tau(t)-\left(\tau_{0}+\left(\frac{W_{0} \tau}{W_{0} \eta}\right) \eta(t)\right)\right| \leqslant C^{\prime \prime}\left|t-t_{0}\right|^{3 / 2}
$$

Since $W_{0} \tau \neq 0$ and $\tau(t)$ is $\mathcal{C}^{\infty}$, this shows that $\eta(t)$ is differentiable from the left at $t_{0}$ and

$$
\begin{aligned}
& \left|\eta(t)-\left(W_{0} \eta\right)\left(t-t_{0}\right)\right| \leqslant C\left|t-t_{0}\right|^{3 / 2}, \\
& W_{0} \eta={ }^{\mathrm{sc}} H_{g} \eta(\tilde{\xi}), \quad \tilde{\xi} \in \hat{\pi}^{-1}\left(\xi_{0}\right) \text { arbitrary. }
\end{aligned}
$$

Using this and the definition of $\omega_{0}$ we also conclude that

$$
\begin{aligned}
& \left|z_{j}(t)-\left(W_{0} z_{j}\right)\left(t-t_{0}\right)\right| \leqslant C\left|t-t_{0}\right|^{3 / 2}, \\
& \left|\nu_{j}(t)-\left(W_{0} \nu_{j}\right)\left(t-t_{0}\right)\right| \leqslant C\left|t-t_{0}\right|^{3 / 2} .
\end{aligned}
$$

This proves the proposition for the $\pi$-invariant functions $\tau, z_{j}, \nu_{j}$ and $\eta$, and indeed it provides a better error estimate. However, we still need to estimate $y_{j}$.

To do so, we consider the second term in $\omega$, see (10.51). Thus, from (A.9),

$$
\left.|| y(t)\right|^{2}-\mu_{0}^{-2} \eta(t)^{2}|\leqslant C| t-\left.t_{0}\right|^{3}, \quad \mu_{0}=\left(\lambda-\tau_{0}^{2}-\tilde{h}\left(z_{0}, \nu_{0}\right)\right)^{1 / 2} .
$$

Taking into account (A.12), we deduce that

$$
r(t)=|y(t)|
$$

satisfies

$$
\left|r(t)^{2}-4 \mu_{0}^{2}\left(t-t_{0}\right)^{2}\right| \leqslant C\left|t-t_{0}\right|^{5 / 2}
$$

Thus,

$$
\left|r(t)+2 \mu_{0}\left(t-t_{0}\right)\right| \leqslant C\left|t-t_{0}\right|^{3 / 2} .
$$

Hence, $r(t)$ is also differentiable from the left at $t_{0}$, and in particular

$$
|y(t)|=r(t) \leqslant C\left|t-t_{0}\right|
$$

Now,

$$
\left|y(t)-\frac{\eta(t)}{\mu_{0}^{2}} \mu_{ \pm}(t)\right|^{2}=|y(t)|^{2}-\frac{\eta(t)^{2}}{\mu_{0}^{2}}-\eta(t)^{2} \frac{\mu_{0}^{2}-\left|\mu_{ \pm}(t)\right|^{2}}{\mu_{0}^{2}} .
$$

By (10.67), (A.10) and (A.19),

$$
\left.|| \mu_{ \pm}(t)\right|^{2}-\mu_{0}^{2}\left|\leqslant C\left(|y(t)|+\omega_{0}(t)^{1 / 2}\right) \leqslant C^{\prime}\right| t-t_{0} \mid .
$$


Thus, by (A.15),

$$
\left|y(t)-\frac{\eta(t)}{\mu_{0}^{2}} \mu_{ \pm}(t)\right|^{2} \leqslant C\left|t-t_{0}\right|^{3} .
$$

In particular, for each $j$ we have

$$
\left|y_{j}(t)-\frac{\eta(t)}{\mu_{0}^{2}} \mu_{j, \pm}(t)\right|^{2} \leqslant C\left|t-t_{0}\right|^{3} .
$$

Let

$$
\theta_{j}=y_{j} / r
$$

so $\theta_{j}$ is a $\pi$-invariant function away from $C_{a}$, and we have $\left|\theta_{j}\right| \leqslant 1$. Also let

$$
\theta_{j}(t)=\frac{y_{j}(t)}{r(t)}, \quad t_{0}-\delta_{1}<t<t_{0}
$$

By the inductive hypothesis, $\theta_{j}(t)$ is differentiable for $t \in\left(t_{0}-\delta_{1}, t_{0}\right)$ from both the left and the right and

$$
\left.\frac{d \theta_{j}}{d t}\right|_{t \pm}=r(t)^{-1} \frac{d y_{j}}{d t}-y_{j}(t) r(t)^{-2} \frac{d r}{d t}
$$

with

$$
d y_{j} /\left.d t\right|_{t \pm}=2 \mu_{j, \pm}(t)
$$

and

$$
d r /\left.d t\right|_{t_{ \pm}}=\frac{1}{2}|y(t)|^{-1}\left(d|y|^{2} /\left.d t\right|_{t_{ \pm}}\right)=2 \frac{\eta(t)}{r(t)} .
$$

Thus,

$$
\left.\frac{d \theta_{j}}{d t}\right|_{t \pm}=2 r(t)^{-1}\left(\mu_{j, \pm}(t)-\frac{y_{j}(t) \eta(t)}{r(t)^{-2}}\right)
$$

so by (A.23) and (A.12),

$$
\left|\frac{d \theta_{j}}{d t}\right|_{t \pm}-2 r(t)^{-1} y_{j}(t)\left(\mu_{0}^{2} \eta(t)\right)^{-1}-\eta(t) r(t)^{-2}|\leqslant C| t-\left.t_{0}\right|^{-1 / 2} .
$$

But, by (A.18) and (A.12), this gives

$$
\left|\frac{d \theta_{j}}{d t}\right|_{t \pm} \leqslant C\left|t-t_{0}\right|^{-1 / 2}
$$

Integrating from $t_{0}-\delta_{1}$ to $t_{0}$ gives that $\theta_{j,-}\left(t_{0}\right)=\lim _{t \rightarrow t_{0}-} \theta_{j}(t)$ exists and

$$
\left|\theta_{j,-}\left(t_{0}\right)-\theta_{j}(t)\right| \leqslant C^{\prime}\left|t-t_{0}\right|^{1 / 2} .
$$

Returning to the original notation, $\theta_{j}=y_{j} / r$, we see that

$$
\left|y_{j}(t)+2 \mu_{0} \theta_{j,-}\left(t_{0}\right)\left(t-t_{0}\right)\right| \leqslant C^{\prime}\left|t-t_{0}\right|^{3 / 2},
$$


so $y_{j}(t)$ is differentiable at $t_{0}$ from the left. We then let

$$
\tilde{\xi}_{-}\left(t_{0}\right)=\left(0, z\left(t_{0}\right), \tau\left(t_{0}\right), \nu\left(t_{0}\right),-\mu_{0} \theta_{j,-}\left(t_{0}\right)\right) .
$$

Then the compositions of the $\pi$-invariant coordinate functions $y_{j}, z_{j}, \tau$ and $\nu_{j}$ with $\gamma$ are all differentiable from the left at $t_{0}$ and the derivative is given by ${ }^{\mathrm{sc}} H_{g}$ applied to the appropriate coordinate function, evaluated at $\tilde{\xi}_{-}(0)$. Note also that from (A.23) and (A.33) we have

$$
\left|\mu_{ \pm}(t)-\mu_{-}\left(t_{0}\right)\right| \leqslant C\left|t-t_{0}\right|^{1 / 2}, \quad t \in\left(t_{0}-\delta_{1}, t_{0}\right) .
$$

Since a general smooth $\pi$-invariant function $f$ has the form

$$
f(y, z, \tau, \mu, \nu)=f_{0}(z, \tau, \nu)+\sum y_{j} f_{j}(z, \tau, \mu, \nu)+\sum y_{j} y_{k} f_{j k}(y, z, \tau, \mu, \nu)
$$

$f_{0}, f_{j}, f_{j k}$ all $\mathcal{C}^{\infty}$, this finishes the proof of the proposition.

\section{REFERENCES}

[1] Bommier A., Propriétés de la matrice de diffusion, Ann. Inst. Henri Poincaré 59 (1993) 237-267.

[2] Dereziński J., Asymptotic completeness of long-range $N$-body quantum systems, Ann. Math. 138 (1993) 427-476.

[3] Derezińs Ki J., GÉRARd C., Scattering Theory of Classical Quantum N-particle Systems, Springer, 1997.

[4] Froese R.G., Herbst I., Exponential bounds, Comm. Math. Phys. 87 (1982) 429-447.

[5] Froese R.G., Herbst I., A new proof of the Mourre estimate, Duke Math. J. 49 (1982) 1075-1085.

[6] Gérard C., Isozaki H., Skibsted E., Commutator algebra resolvent estimates, in: Advanced Studies in Pure Mathematics, Vol. 23, 1994, pp. 69-82.

[7] GÉrARd C., IsozaKi H., SkibSted E., N-body resolvent estimates, J. Math. Soc. Japan 48 (1996) $135-160$.

[8] Graf G.M., Asymptotic completeness for $N$-body short range systems, Comm. Math. Phys. 132 (1990) 73-101.

[9] Hassell A., VASY A., Symbolic functional calculus, J. Funct. Anal. 173 (2000) 257-283.

[10] HelfFER B., S JöSTRAND J., Équation de Schrödinger avec champ magnétique et équation de Harper.

[11] Hörmander L., Fourier integral operators, Acta Mathematica 127 (1971) 79-183.

[12] Hörmander L., The Analysis of Linear Partial Differential Operators, Vols. 1-4, Springer-Verlag, 1983.

[13] Ikawa M. (Ed.), Spectral and Scattering Theory, Marcel Dekker, 1994.

[14] Isozaki H., Structures of S-matrices for three body Schrödinger operators, Comm. Math. Phys. 146 (1992) 241-258.

[15] IsOzAKI H., A generalization of the radiation condition of Sommerfeld for $N$-body Schrödinger operators, Duke Math. J. 74 (1994) 557-584.

[16] Isozaki H., A uniqueness theorem for the $N$-body Schrödinger equation and its applications, in: Ikawa M. (Ed.), Spectrial and Scattering Theory, Marcel Dekker, 1994.

[17] Jensen A., Propagation estimates for Schrödinger-type operators, Trans. Amer. Math. Soc. 291 (1) (1985) 129-144.

[18] Lebeau G., Propagation des ondes dans les variétés à coins, Ann. Scient. Éc. Norm. Sup. 30 (1997) 429-497.

[19] Mazzeo R., Melrose R.B., Pseudodifferential operators on manifolds with fibred boundaries, Asian J. Math. 2 (1998).

[20] Melrose R.B., Differential analysis on manifolds with corners, In preparation. 
[21] Melrose R.B., Calculus of conormal distributions on manifolds with corners, Internat. Math. Res. Notices 3 (1992) 51-61.

[22] Melrose R.B., Spectral and scattering theory for the Laplacian on asymptotically Euclidian spaces, in: Ikawa M. (Ed.), Spectrial and Scattering Theory, Marcel Dekker, 1994.

[23] Melrose R.B., Sjöstrand J., Singularities of boundary value problems. I, Comm. Pure Appl. Math. 31 (1978) 593-617.

[24] Melrose R.B., Sjöstrand J., Singularities of boundary value problems. II, Comm. Pure Appl. Math. 35 (1982) 129-168.

[25] Melrose R.B., Zworski M., Scattering metrics, Inventiones Math. 124 (1996) 389-436.

[26] Mourre E., Absence of singular continuous spectrum of certain self-adjoint operators, Comm. Math. Phys. 78 (1981) 391-408.

[27] Perry P., Sigal I.M., Simon B., Spectral analysis of N-body Schrödinger operators, Ann. Math. 114 (1981) 519-567.

[28] Sigal I.M., Soffer A., N-particle scattering problem, Ann. Math. 125 (1987) 35-108.

[29] Sigal I.M., SOFFER A., Long-range many-body scattering, Inventiones Math. 99 (1990) 115-143.

[30] Sigal I.M., SofFER A., Asymptotic completeness of $N \leqslant 4$-particle systems with the Coulomb-type interactions, Duke Math. J. 71 (1993) 243-298.

[31] Sigal I.M., Soffer A., Asymptotic completeness of $N$-particle long-range scattering, J. Amer. Math. Soc. 7 (1994) 307-334.

[32] SjÖstrand J., Propagation of analytic singularities for second order Dirichlet problems, Comm. PDE 5 (1980) 41-94.

[33] Skibsted E., Smoothness of N-body scattering amplitudes, Rev. Math. Phys. 4 (1992) 619-658.

[34] Taylor M., Grazing rays, Comm. Pure Appl. Math. 29 (1976) 1-38.

[35] VASY A., Asymptotic behavior of generalized eigenfunctions in $N$-body scattering, J. Funct. Anal. 148 (1997) 170-184.

[36] VASY A., Propagation of singularities in three-body scattering, Ph.D. Thesis, Massachusetts Institute of Technology, 1997.

[37] VASY A., Structure of the resolvent for three-body potentials, Duke Math. J. 90 (1997) 379-434.

[38] VASY A., Geometric scattering theory for long-range potentials, Int. Math. Res. Notices 1998 (1998) $285-315$.

[39] VASY A., Scattering matrices in many-body scattering, Comm. Math. Phys. 200 (1999) 105-124.

[40] VASY A., Propagation of singularities in three-body scattering, Astérisque 262 (2000).

[41] Wang X.P., Microlocal estimates for N-body Schrödinger operators, J. Fac. Sci. Univ. Tokyo Sect. IA, Math. 40 (1993) 337-385.

[42] Yafaev D., Radiation conditions, Comm. Math. Phys. 154 (1993) 523-554.

(Manuscript received March 19, 1999; accepted, after revision, September 14, 2000.)

\author{
András VASY \\ Department of Mathematics, \\ University of California, \\ Berkeley, CA 94720-3840, USA \\ E-mail: andras@math.mit.edu
}

Current address:

Department of Mathematics,

Massachusetts Institute of Technology,

Cambridge, MA 02139, USA 